\title{
Asymmetric Synthesis of 5,7-Fused Ring System Enabled by Intra-molecular Buchner Reaction with Chiral Rhodium Catalyst
}

Takayuki Hoshi,${ }^{\dagger}$ Eisuke Ota,${ }^{\dagger}$ Yasuhide Inokuma,${ }^{\dagger}$ and Junichiro Yamaguchi ${ }^{\dagger *}$

†Department of Applied Chemistry, Waseda University, 3-4-1 Ohkubo, Shinjuku, Tokyo 169-8555, Japan $\$$ Division of Applied Chemistry, Faculty of Engineering, Hokkaido University, Kita 13 Nishi 8 Kita-ku, Sapporo,

Hokkaido 060-8628, Japan.

E-mail: junyamaguchi@waseda.jp

\section{Table of Contents}

1. General

S2

2. Preparation of $\alpha$-diazoesters

S3-S17

3. Rh-catalyzed asymmetric intramolecular Buchner ring expansion of $\alpha$-diazoester

4. $\quad{ }^{1} \mathrm{H}$ and ${ }^{13} \mathrm{C}$ NMR Spectra

S18-S25

5. HPLC spectra

S26-S125

6. Single Crystal X-ray Analysis

S126-S149

7. References

S150-S151

S152 
Supporting Information (Hoshi, Ota, Inokuma, Yamaguchi) Asymmetric Synthesis of 5,7-Fused Ring System Enabled by Intra-molecular Buchner with Chiral Rhodium Catalyst

\section{General}

Unless otherwise noted, all reactants or reagents including anhydrous solvents were obtained from commercial suppliers and used as received. $\mathrm{Rh}_{2}(\mathrm{OAc})_{4}, \mathrm{Rh}_{2}(\mathrm{TPA})_{4}, \mathrm{Rh}_{2}(S \text {-PTPA })_{4}, \mathrm{Rh}_{2}(S \text {-PTTL })_{4}$ and $\mathrm{Rh}_{2}(S \text {-TCPTTL })_{4}$ were obtained from TCI Chemical. $\mathrm{Rh}_{2}(\mathrm{esp})_{2}$ was obtained from Sigma-Aldrich. $\mathrm{Rh}_{2}(\mathrm{OPiv})_{4}{ }^{[1]}$ and $\mathrm{Rh}_{2}(S \text {-TCPTAD })_{4}{ }^{[2]}$ were synthesized according to procedures reported in the literature and the spectra matched with those of compounds reported in the literature. All Buchner ring expansions were performed in $25-\mathrm{mL}$ glass vessel tubes equipped with a screw cap in an ice-water bath. All reactions with heating were performed with $25-\mathrm{mL}$ glass vessel tubes equipped with a screw cap and heated (IKA Plate RCT digital) in an oil-bath or a 12-well aluminum reaction block (IKA DB4.9). All work-up and purification procedures were carried out with reagent-grade solvents in air.

Analytical thin-layer chromatography (TLC) was performed using Silica-gel 70 TLC Plate-Wako (0.25 mm). The developed chromatogram was analyzed by UV lamp $(254 \mathrm{~nm})$. Flash column chromatography was performed with Biotage Isolera ${ }^{\circledR}$ equipped with Biotage SNAP Cartridge KP-Sil columns and hexane/EtOAc as an eluent. High-resolution mass spectra were conducted on Thermo Fisher Scientific ExactivePlus (ESI and DART). Chiral high performance liquid chromatography (HPLC) was performed using SHIMADZU Prominence-i LC-2030C Plus ${ }^{\circledR}$ equipped with DAICEL Chiralcel $^{\circledR}$. Details of chromatographic conditions on the separation of the products are described with compound data in Section 3. Optical rotations were measured with a JASCO P-2200. X-ray crystallographic data were obtained using Rigaku XtaLAB Synergy-R/DW (for compound 2A) equipped with a HyPix-6000HE detector or Rigaku XtaLAB P200 (for compound 8E) diffractometers equipped with a PILATUS200K. Nuclear magnetic resonance (NMR) spectra were recorded on a JEOL JNM-ECS-400 ( $\left({ }^{1} \mathrm{H} 400 \mathrm{MHz},{ }^{13} \mathrm{C} 101 \mathrm{MHz}\right)$. Chemical shifts for ${ }^{1} \mathrm{H}$ NMR are expressed in parts per million (ppm) relative to tetramethylsilane $(\delta 0.00 \mathrm{ppm})$. Chemical shifts for ${ }^{13} \mathrm{C}$ NMR are expressed in ppm relative to $\mathrm{CDCl}_{3}(\delta 77.16 \mathrm{ppm})$. Data are reported as follows: chemical shift, multiplicity $(\mathrm{s}=$ singlet, $\mathrm{d}=$ doublet, $\mathrm{dd}=$ doublet of doublets, $\mathrm{ddd}=$ doublet of doublets of doublets, $\mathrm{t}$ $=$ triplet, $\mathrm{dt}=$ doublet of triplets, $\mathrm{td}=$ triplet of doublets, $\mathrm{q}=$ quartet, $\mathrm{dq}=$ doublet of quartets, $\mathrm{m}=$ multiplet), coupling constant $(\mathrm{Hz})$, and integration. 


\section{Preparation of $\alpha$-diazoesters}

\section{2-1. Synthesis of $\alpha$-diazoesters $1 \mathrm{~A}-\mathrm{F}$ and $4^{[3]}$}

\section{General procedure}
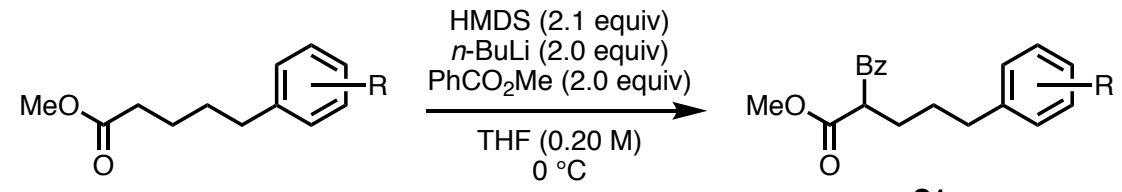

S1
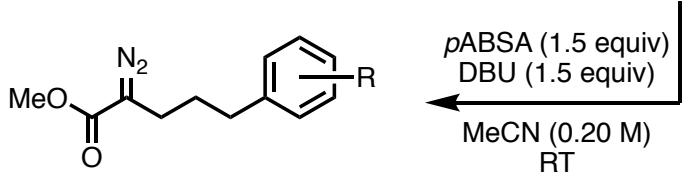

1 or 4

A 50-mL round bottom flask containing a magnetic stirring bar was dried with a heat-gun in vacuo and filled with $\mathrm{N}_{2}$ after cooling to room temperature. To the flask were added anhydrous THF $(0.40$ M) and bis(trimethylsilyl)amine (HMDS, 2.1 equiv). To the solution was slowly added $n$-BuLi (1.55 $\mathrm{M}$ in hexane, 2.0 equiv) at $0{ }^{\circ} \mathrm{C}$. After stirring for 10 minutes, methyl benzoate (2.0 equiv) and methyl 5-arylpentanoate $\left(0.40 \mathrm{M}\right.$ in anhydrous THF) were added at $0{ }^{\circ} \mathrm{C}$. The solution was stirred for 10 minutes and then poured into sat. $\mathrm{NH}_{4} \mathrm{Cl}$ aq. The resulting mixture was extracted two times with EtOAc, dried over $\mathrm{Na}_{2} \mathrm{SO}_{4}$, filtrated, and concentrated in vacuo. The product $\mathbf{S 1}$ was used for the next step without further purification.

To a solution of $\mathbf{S 1}$ in $\mathrm{MeCN}(0.20 \mathrm{M})$ were added $p$-acetamidobenzenesulfonyl azide ( $p$ ABSA, 1.5 equiv) and 1,8-diazabicyclo[5.4.0] undec-7-ene (DBU, 1.5 equiv) at $0{ }^{\circ} \mathrm{C}$. After stirring the mixture for 3 hours at room temperature, the reaction was quenched with water. The organics were extracted two times with ether, dried over $\mathrm{Na}_{2} \mathrm{SO}_{4}$, filtrated and concentrated in vacuo. The residue was purified by Isolera ${ }^{\circledR}$ (hexane/EtOAc 95:5 to 90:10) to afford corresponding $\alpha$-diazoester.

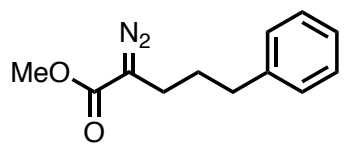

\section{Methyl 2-diazo-5-phenylpentanoate (1A $)^{[4]}$}

Following general procedure using $1.42 \mathrm{~g}$ of methyl 5-phenylvalerate, 1A was obtained as a yellow oil $(1.27 \mathrm{~g}, 5.82 \mathrm{mmol}, 79 \%$ yield in 2 steps). The spectra are in accordance with those of the compound reported in the literature; $\mathrm{R}_{\mathrm{f}}$ value $=0.40$ (hexane/EtOAc 9:1).

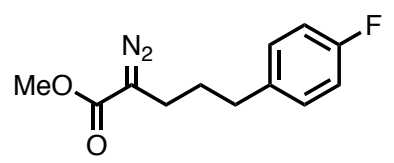

\section{Methyl 2-diazo-5-(4-fluorophenyl)pentanoate (1B)}

Following general procedure using $1.12 \mathrm{~g}$ of methyl 5-(4-fluorophenyl)pentanoate, 1B was obtained as a yellow oil (1.06 g, $4.49 \mathrm{mmol}, 85 \%$ yield in 2 steps). ${ }^{1} \mathrm{H}$ NMR $\left(400 \mathrm{MHz}, \mathrm{CDCl}_{3}\right) \delta$ 7.15-7.09 (m, 
2H), 6.99-6.92 (m, 2H), $3.75(\mathrm{~s}, 3 \mathrm{H}), 2.64(\mathrm{t}, J=7.6 \mathrm{~Hz}, 2 \mathrm{H}), 2.33(\mathrm{t}, J=7.6 \mathrm{~Hz}, 2 \mathrm{H}), 1.82$ (quintet, $J$ $=7.6 \mathrm{~Hz}, 2 \mathrm{H}) ;{ }^{13} \mathrm{C} \mathrm{NMR}\left(101 \mathrm{MHz}, \mathrm{CDCl}_{3}\right) \delta 167.9,161.4\left(\mathrm{~d}, J_{\mathrm{C}-\mathrm{F}}=247.8 \mathrm{~Hz}\right), 137.0\left(\mathrm{~d}, J_{\mathrm{C}-\mathrm{F}}=2.9\right.$ $\mathrm{Hz}), 129.8\left(\mathrm{~d}, J_{\mathrm{C}-\mathrm{F}}=7.7 \mathrm{~Hz}\right), 115.2\left(\mathrm{~d}, J_{\mathrm{C}-\mathrm{F}}=21.2 \mathrm{~Hz}\right), 51.9,34.1,29.4,22.7\left(\mathrm{C}=\mathrm{N}_{2}\right.$ carbon was not observed); HRMS (ESI) $m / z$ calcd for $\mathrm{C}_{12} \mathrm{H}_{13} \mathrm{FO}_{2} \mathrm{~N}_{2} \mathrm{Na}[\mathrm{M}+\mathrm{Na}]^{+}: 259.0853$ found 259.0850; $\mathrm{R}_{\mathrm{f}}$ value $=0.57($ hexane $/$ EtOAc 5:1).

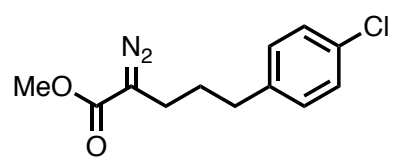

\section{Methyl 2-diazo-5-(4-chlorophenyl)pentanoate (1C)}

Following general procedure using $1.46 \mathrm{~g}$ of methyl 5-(4-chlorophenyl)pentanoate, 1C was obtained as a yellow oil (1.43 g, $5.66 \mathrm{mmol}, 88 \%$ yield in 2 steps). ${ }^{1} \mathrm{H}$ NMR (400 MHz, $\left.\mathrm{CDCl}_{3}\right) \delta 7.24$ (d, $J=8.4 \mathrm{~Hz}, 2 \mathrm{H}), 7.10$ (d, $J=8.4 \mathrm{~Hz}, 2 \mathrm{H}), 3.75$ (s, 3H), 2.63 (t, $J=7.6 \mathrm{~Hz}, 2 \mathrm{H}), 2.32$ (t, $J=7.6 \mathrm{~Hz}$, $2 \mathrm{H}$ ), 1.82 (quintet, $J=7.6 \mathrm{~Hz}, 2 \mathrm{H}) ;{ }^{13} \mathrm{C} \mathrm{NMR}\left(101 \mathrm{MHz}, \mathrm{CDCl}_{3}\right) \delta 167.9,139.8,131.8,129.8,128.6$, 52.0, 34.2, 29.2, $22.8\left(\mathrm{C}=\mathrm{N}_{2}\right.$ carbon was not observed); HRMS (ESI) $m / z$ calcd for $\mathrm{C}_{12} \mathrm{H}_{13} \mathrm{ClO}_{2} \mathrm{~N}_{2} \mathrm{Na}$ $[\mathrm{M}+\mathrm{Na}]^{+}: 275.0558$ found 275.0554; $\mathrm{R}_{\mathrm{f}}$ value $=0.37$ (hexane/EtOAc 9:1).

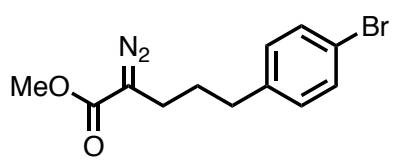

\section{Methyl 2-diazo-5-(4-bromophenyl)pentanoate (1D)}

Following general procedure using $807.8 \mathrm{mg}$ of methyl 5-(4-bromophenyl)pentanoate, 1D was obtained as a yellow oil ( $855.8 \mathrm{mg}, 2.88 \mathrm{mmol}, 97 \%$ yield in 2 steps). ${ }^{1} \mathrm{H}$ NMR (400 MHz, $\left.\mathrm{CDCl}_{3}\right) \delta$ 7.40 (d, $J=8.4 \mathrm{~Hz}, 2 \mathrm{H}), 7.05$ (d, $J=8.4 \mathrm{~Hz}, 2 \mathrm{H}), 3.76$ (s, 3H), 2.63 (t, $J=7.6 \mathrm{~Hz}, 2 \mathrm{H}), 2.33$ (t, $J=7.6$ $\mathrm{Hz}, 2 \mathrm{H}$ ), 1.82 (quintet, $J=7.6 \mathrm{~Hz}, 2 \mathrm{H}$ ); ${ }^{13} \mathrm{C} \mathrm{NMR}\left(101 \mathrm{MHz}, \mathrm{CDCl}_{3}\right) \delta 167.8,140.3,131.5,130.2$, 119.8, 52.0, 34.3, 29.2, $22.8\left(\mathrm{C}=\mathrm{N}_{2}\right.$ carbon was not observed); HRMS (ESI) $\mathrm{m} / \mathrm{z}$ calcd for $\mathrm{C}_{12} \mathrm{H}_{13} \mathrm{BrO}_{2} \mathrm{~N}_{2} \mathrm{Na}[\mathrm{M}+\mathrm{Na}]^{+}: 319.0053$ found 319.0050; $\mathrm{R}_{\mathrm{f}}$ value $=0.46$ (hexane/EtOAc 5:1).

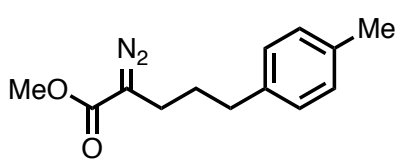

\section{Methyl 2-diazo-5-(p-tolyl)pentanoate (1E)}

Following general procedure using $552.5 \mathrm{mg}$ of methyl 5-(p-tolyl)pentanoate, 1E was obtained as a yellow oil (499.0 mg, $2.15 \mathrm{mmol}, 80 \%$ yield in 2 steps). ${ }^{1} \mathrm{H}$ NMR (400 MHz, $\left.\mathrm{CDCl}_{3}\right) \delta$ 7.12-7.04 (m, $4 \mathrm{H}), 3.76(\mathrm{~s}, 3 \mathrm{H}), 2.64(\mathrm{t}, J=7.6 \mathrm{~Hz}, 2 \mathrm{H}), 2.36-2.30(\mathrm{~m}, 2 \mathrm{H}), 2.32(\mathrm{~s}, 3 \mathrm{H}), 1.82$ (quintet, $J=7.6 \mathrm{~Hz}$, $2 \mathrm{H}) ;{ }^{13} \mathrm{C} \mathrm{NMR}\left(101 \mathrm{MHz}, \mathrm{CDCl}_{3}\right) \delta 138.3,135.5,129.2,128.3,51.9,34.5,29.4,22.8,21.1(\mathrm{C}=\mathrm{O}$ and $\mathrm{C}=\mathrm{N}_{2}$ carbons were not observed); HRMS (ESI) $\mathrm{m} / z$ calcd for $\mathrm{C}_{13} \mathrm{H}_{16} \mathrm{O}_{2} \mathrm{~N}_{2} \mathrm{Na}[\mathrm{M}+\mathrm{Na}]^{+}: 255.1104$ found 255.1100; $\mathrm{R}_{\mathrm{f}}$ value $=0.42$ (hexane/EtOAc 9:1). 
<smiles>COC(=O)C(=N)CCCc1ccc(OC)cc1</smiles>

\section{Methyl 2-diazo-5-(4-methoxyphenyl)pentanoate (1F)}

Following general procedure using $659.9 \mathrm{mg}$ of methyl 5-(4-methoxyphenyl)pentanoate, 1F was obtained as a yellow oil (590.6 mg, $2.38 \mathrm{mmol}, 80 \%$ yield in 2 steps). ${ }^{1} \mathrm{H}$ NMR (400 MHz, $\left.\mathrm{CDCl}_{3}\right) \delta$ $7.09(\mathrm{~d}, J=8.8 \mathrm{~Hz}, 2 \mathrm{H}), 6.83(\mathrm{~d}, J=8.8 \mathrm{~Hz}, 2 \mathrm{H}), 3.79(\mathrm{~s}, 3 \mathrm{H}), 3.76(\mathrm{~s}, 3 \mathrm{H}), 2.62(\mathrm{t}, J=7.6 \mathrm{~Hz}, 2 \mathrm{H})$, $2.33(\mathrm{t}, J=7.6 \mathrm{~Hz}, 2 \mathrm{H}), 1.81$ (quintet, $J=7.6 \mathrm{~Hz}, 2 \mathrm{H}) ;{ }^{13} \mathrm{C} \mathrm{NMR}\left(101 \mathrm{MHz}, \mathrm{CDCl}_{3}\right) \delta 157.9,133.3$, 129.3, 113.8, 55.2, 51.9, 33.9, 29.5, $22.6\left(\mathrm{C}=\mathrm{O}\right.$ and $\mathrm{C}=\mathrm{N}_{2}$ carbons were not observed); HRMS (ESI) $m / z$ calcd for $\mathrm{C}_{13} \mathrm{H}_{16} \mathrm{O}_{3} \mathrm{~N}_{2} \mathrm{Na}[\mathrm{M}+\mathrm{Na}]^{+}: 271.1053$ found $271.1051 ; \mathrm{R}_{\mathrm{f}}$ value $=0.38$ (hexane/EtOAc $5: 1)$.

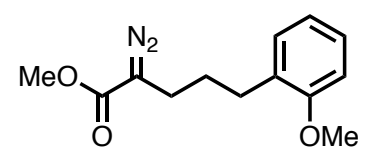

\section{Methyl 2-diazo-5-(2-methoxy)pentanoate (4)}

Following general procedure using $238.5 \mathrm{mg}$ of methyl 5-(2-methoxyphenyl)pentanoate, 4 was obtained as a yellow oil $\left(215.7 \mathrm{~g}, 0.869 \mathrm{mmol}, 81 \%\right.$ yield in 2 steps). ${ }^{1} \mathrm{H}$ NMR $\left(400 \mathrm{MHz}, \mathrm{CDCl}_{3}\right) \delta$ $7.18(\mathrm{td}, J=7.6,1.6 \mathrm{~Hz}, 1 \mathrm{H}), 7.12(\mathrm{dd}, J=7.6,1.6 \mathrm{~Hz}, 1 \mathrm{H}), 6.88(\mathrm{t}, J=7.6 \mathrm{~Hz}, 1 \mathrm{H}), 6.84(\mathrm{~d}, J=7.6$ $\mathrm{Hz}, 1 \mathrm{H}), 3.81(\mathrm{~s}, 3 \mathrm{H}), 3.76(\mathrm{~s}, 3 \mathrm{H}), 2.67(\mathrm{t}, J=7.6 \mathrm{~Hz}, 2 \mathrm{H}), 2.35$ (t, $J=7.6 \mathrm{~Hz}, 2 \mathrm{H}), 1.80$ (quintet, $J=$ $7.6 \mathrm{~Hz}, 2 \mathrm{H}) ;{ }^{13} \mathrm{C} \mathrm{NMR}\left(101 \mathrm{MHz}, \mathrm{CDCl}_{3}\right) \delta 157.5,130.0,129.8,127.3,120.5,110.3,55.2,51.9,29.4$, 27.6, $22.8\left(\mathrm{C}=\mathrm{O}\right.$ and $\mathrm{C}=\mathrm{N}_{2}$ carbons were not observed); HRMS (ESI) $m / z$ calcd for $\mathrm{C}_{13} \mathrm{H}_{16} \mathrm{O}_{3} \mathrm{~N}_{2} \mathrm{Na}$ $[\mathrm{M}+\mathrm{Na}]^{+}: 271.1053$ found $271.1051 ; \mathrm{R}_{\mathrm{f}}$ value $=0.48($ hexane/EtOAc $4: 1)$. 


\section{2-2. Synthesis of $\alpha$-diazoesters 7A-K}

1) Scheme S1: Synthesis of 3-substituted $\alpha$-diazoesters $7 A-K$
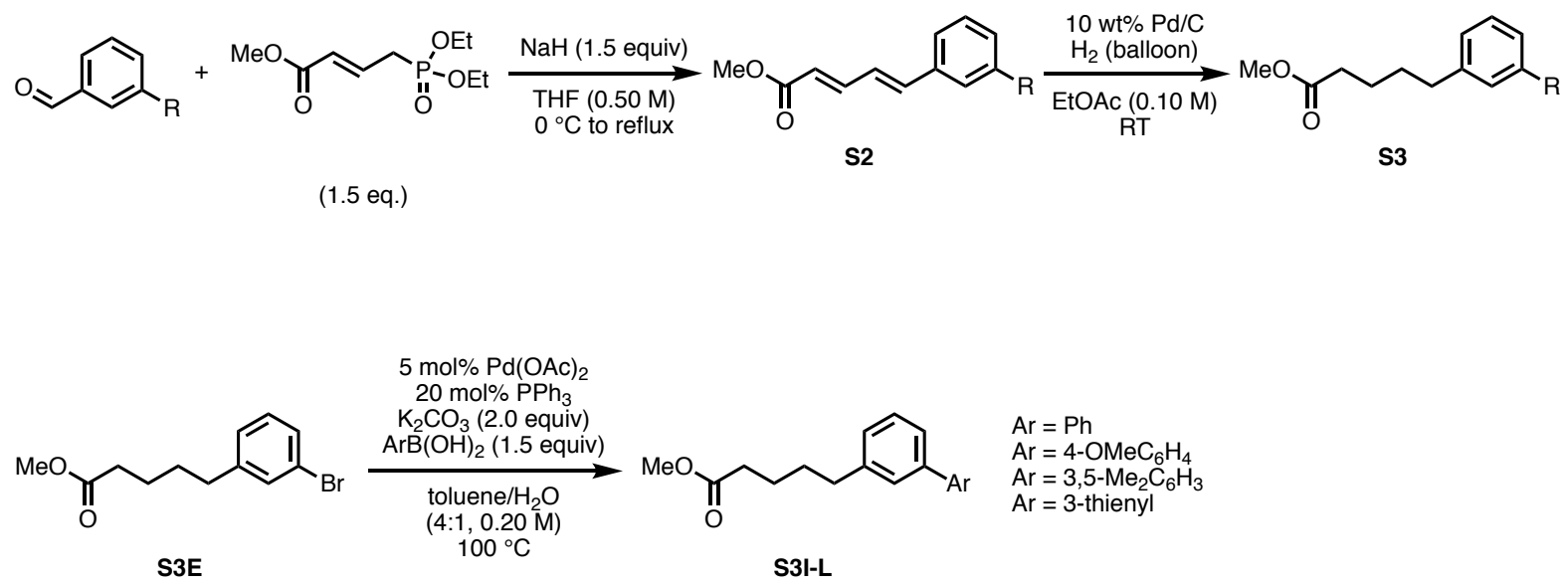

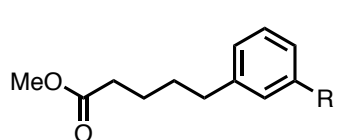

S3

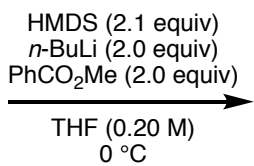
$0{ }^{\circ} \mathrm{C}$

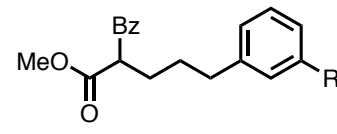

S4

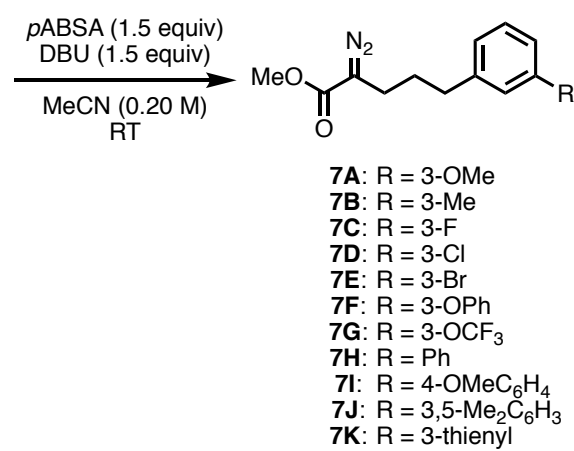

\section{2) Synthesis of $\alpha, \beta, \gamma, \delta$-unsaturated esters $\mathrm{S} 2$}

\section{General procedure}

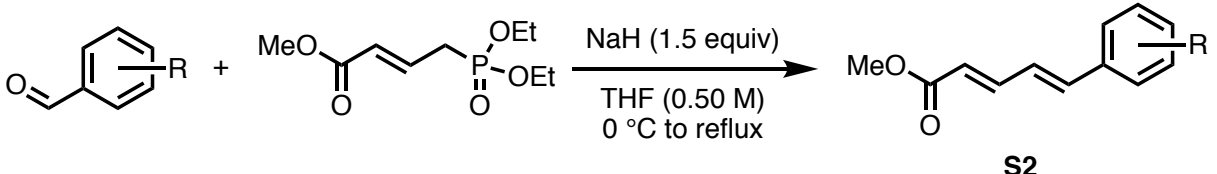

(1.5 eq.)

A $25-\mathrm{mL}$ glass vessel equipped with a screw cap containing a magnetic stirring bar was dried with a heat-gun in vacuo and filled with $\mathrm{N}_{2}$ after cooling to room temperature. To this vessel were added anhydrous THF $(5 \mathrm{~mL})$, benzaldehyde $(2.5 \mathrm{mmol}, 1.0$ equiv $)$ and methyl (E)-4-(diethoxyphosphoryl)but-2-enoate ${ }^{[5]}$ (354.3 mg, $3.75 \mathrm{mmol}, 1.5$ equiv). To the solution was added $\mathrm{NaH}\left(60 \%\right.$ dispersion in mineral oil, $60.0 \mathrm{mg}, 1.5$ equiv) portionwise at $0{ }^{\circ} \mathrm{C}$. After the completion of addition of $\mathrm{NaH}$, the vessel was sealed with a screw cap and then heated at $80{ }^{\circ} \mathrm{C}$ overnight. The reaction was quenched with sat. $\mathrm{NH}_{4} \mathrm{Cl}$ aq. and extracted three times with EtOAc. The combined organic layer was dried over $\mathrm{Na}_{2} \mathrm{SO}_{4}$, filtrated, and concentrated in vacuo. The residue was 
purified by Isolera ${ }^{\circledR}$ (hexane/EtOAc $95: 5$ to $90: 10$ ) to afford the corresponding $\alpha, \beta, \gamma, \delta$-unsaturated ester S2.

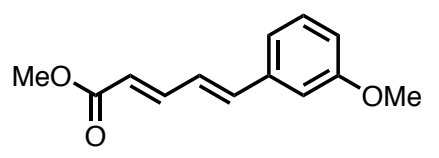

\section{Methyl (2E,4E)-5-(3-methoxyphenyl)penta-2,4-dienoate (S2A)}

Following general procedure, S2A was obtained as a white solid (351.4 mg, $1.61 \mathrm{mmol}, 64 \%$ yield). ${ }^{1} \mathrm{H}$ NMR $\left(400 \mathrm{MHz}, \mathrm{CDCl}_{3}\right) \delta 7.44(\mathrm{ddd}, J=15.2,6.8,3.6 \mathrm{~Hz}, 1 \mathrm{H}), 7.31-7.23(\mathrm{~m}, 1 \mathrm{H}), 7.06(\mathrm{~d}, J=$ $8.0 \mathrm{~Hz}, 1 \mathrm{H}), 6.98(\mathrm{~s}, 1 \mathrm{H}), 6.91-6.80(\mathrm{~m}, 3 \mathrm{H}), 5.99(\mathrm{~d}, J=15.2 \mathrm{~Hz}, 1 \mathrm{H}), 3.83(\mathrm{~s}, 3 \mathrm{H}), 3.77(\mathrm{~s}, 3 \mathrm{H}) ;{ }^{13} \mathrm{C}$ NMR (101 MHz, $\left.\mathrm{CDCl}_{3}\right) \delta 167.6,160.0,144.8,140.6,137.5,129.9,126.6,121.1,120.1,115.0,112.4$, 55.4, 51.7; HRMS (ESI) $\mathrm{m} / z$ calcd for $\mathrm{C}_{13} \mathrm{H}_{15} \mathrm{O}_{3}[\mathrm{M}+\mathrm{H}]^{+}: 219.1016$ found 219.1014; $\mathrm{R}_{\mathrm{f}}$ value $=0.33$ (hexane/EtOAc 5:1).

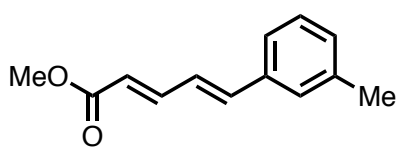

\section{Methyl (2E,4E)-5-(m-tolyl)penta-2,4-dienoate (S2B) ${ }^{[6]}$}

Following general procedure, S2B was obtained as a white solid (291.5 mg, $1.44 \mathrm{mmol}, 58 \%$ yield). The spectra are in accordance with those of the compound reported in the literature. $R_{\mathrm{f}}$ value $=0.39$ (hexane/EtOAc 9:1).

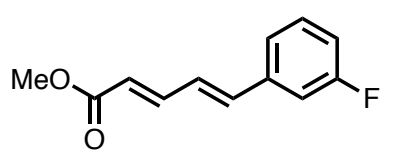

\section{Methyl (2E,4E)-5-(3-fluorophenyl)penta-2,4-dienoate (S2C)}

Following general procedure, $\mathbf{S 2 C}$ was obtained as a white solid (284.1 mg, $1.38 \mathrm{mmol}, 55 \%$ yield). ${ }^{1} \mathrm{H}$ NMR $\left(400 \mathrm{MHz}, \mathrm{CDCl}_{3}\right) \delta 7.43(\mathrm{ddd}, J=15.6,6.0,4.0 \mathrm{~Hz}, 1 \mathrm{H}), 7.32(\mathrm{td}, J=8.0,6.0 \mathrm{~Hz}, 1 \mathrm{H})$, $7.22(\mathrm{dt}, J=8.0,0.8 \mathrm{~Hz}, 1 \mathrm{H}), 7.19-7.14(\mathrm{~m}, 1 \mathrm{H}), 7.00$ (tdd, $J=8.0,2.4,0.8 \mathrm{~Hz}, 1 \mathrm{H}) 6.88-6.84(\mathrm{~m}, 2 \mathrm{H})$, $6.03(\mathrm{~d}, J=15.6 \mathrm{~Hz}, 1 \mathrm{H}), 3.78(\mathrm{~s}, 3 \mathrm{H}) ;{ }^{13} \mathrm{C}$ NMR $\left(101 \mathrm{MHz}, \mathrm{CDCl}_{3}\right) \delta 167.5,163.2\left(\mathrm{~d}, J_{\mathrm{C}-\mathrm{F}}=249.6\right.$ $\mathrm{Hz}), 144.4,139.2\left(\mathrm{~d}, J_{\mathrm{C}-\mathrm{F}}=2.9 \mathrm{~Hz}\right), 138.4\left(\mathrm{~d}, J_{\mathrm{C}-\mathrm{F}}=7.7 \mathrm{~Hz}\right), 130.4\left(\mathrm{~d}, J_{\mathrm{C}-\mathrm{F}}=8.7 \mathrm{~Hz}\right), 127.5,123.3$, 121.9, $116.0\left(\mathrm{~d}, J_{\mathrm{C}-\mathrm{F}}=22.3 \mathrm{~Hz}\right), 113.5\left(\mathrm{~d} J_{\mathrm{C}-\mathrm{F}}=22.2 \mathrm{~Hz}\right), 51.8$; HRMS (DART) $\mathrm{m} / \mathrm{z}$ calcd for $\mathrm{C}_{12} \mathrm{H}_{12} \mathrm{FO}_{2}[\mathrm{M}+\mathrm{H}]^{+}: 207.0816$ found 207.0813; $\mathrm{R}_{\mathrm{f}}$ value $=0.50$ (hexane/EtOAc 5:1).

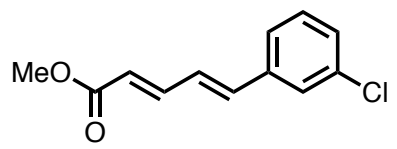

Methyl (2E,4E)-5-(3-chlorophenyl)penta-2,4-dienoate (S2D) ${ }^{[6]}$ 
Following general procedure, S2D was obtained as a white solid (377.9 mg, $1.70 \mathrm{mmol}, 68 \%$ yield). The spectra are in accordance with those of the compound reported in the literature. $R_{f}$ value $=0.35$ (hexane/EtOAc 9:1).

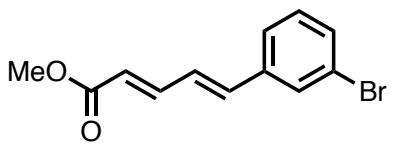

\section{Methyl (2E,4E)-5-(3-bromophenyl)penta-2,4-dienoate (S2E)}

Following general procedure, S2E was obtained as a white solid (338.6 mg, $1.27 \mathrm{mmol}, 51 \%$ yield). ${ }^{1} \mathrm{H}$ NMR $\left(400 \mathrm{MHz}, \mathrm{CDCl}_{3}\right) \delta 7.61(\mathrm{~s}, 1 \mathrm{H}), 7.47-7.34(\mathrm{~m}, 3 \mathrm{H}), 7.22(\mathrm{t}, J=8.0 \mathrm{~Hz}, 1 \mathrm{H}), 6.90-6.78(\mathrm{~m}$, $2 \mathrm{H}), 6.02(\mathrm{~d}, J=15.6 \mathrm{~Hz}, 1 \mathrm{H}), 3.78(\mathrm{~s}, 3 \mathrm{H}) ;{ }^{13} \mathrm{C} \mathrm{NMR}\left(101 \mathrm{MHz}, \mathrm{CDCl}_{3}\right) \delta 167.5,144.3,138.8,138.2$, $131.9,130.4,130.0,127.6,126.0,123.1,122.0,51.8$; HRMS (DART) $\mathrm{m} / z$ calcd for $\mathrm{C}_{12} \mathrm{H}_{12} \mathrm{BrO}_{2}$ $[\mathrm{M}+\mathrm{H}]^{+}: 267.0015$ found 267.0009; $\mathrm{R}_{\mathrm{f}}$ value $=0.44$ (hexane/EtOAc 5:1).<smiles>COC(=O)/C=C/C=C/c1cccc(Oc2ccccc2)c1</smiles>

\section{Methyl (2E,4E)-5-(3-bromophenyl)penta-2,4-dienoate (S2F)}

Following general procedure, S2F was obtained as a white solid (426.2 $\mathrm{mg}, 1.52 \mathrm{mmol}, 61 \%$ yield). ${ }^{1} \mathrm{H}$ NMR (400 MHz, $\mathrm{CDCl}_{3}$ ) $\delta 7.42(\mathrm{ddd}, J=15.6,8.0,2.0 \mathrm{~Hz}, 1 \mathrm{H}), 7.38-7.28(\mathrm{~m}, 3 \mathrm{H}), 7.19$ (d, $J=$ $7.6 \mathrm{~Hz}, 1 \mathrm{H}), 7.15-7.09$ (m, 2H), 7.02 (dd, $J=8.8,0.8 \mathrm{~Hz}, 2 \mathrm{H}), 6.95$ (ddd, $J=8.8,2.8,0.8 \mathrm{~Hz}, 1 \mathrm{H}$ ), 6.88-6.77 (m, 2H), $5.98(\mathrm{~d}, J=15.6 \mathrm{~Hz}, 1 \mathrm{H}), 3.76(\mathrm{~s}, 3 \mathrm{H}) ;{ }^{13} \mathrm{C} \mathrm{NMR}\left(101 \mathrm{MHz}, \mathrm{CDCl}_{3}\right) \delta 167.5$, 157.8, 157.1, 144.6, 139.9, 138.0, 130.2, 130.0, 127.0, 123.6, 122.4, 121.4, 119.6, 119.1, 117.2, 51.7; HRMS (DART) $m / z$ calcd for $\mathrm{C}_{18} \mathrm{H}_{17} \mathrm{O}_{3}[\mathrm{M}+\mathrm{H}]^{+}: 281.1172$ found $281.1166 ; \mathrm{R}_{\mathrm{f}}$ value $=0.27$ (hexane/EtOAc 9:1).

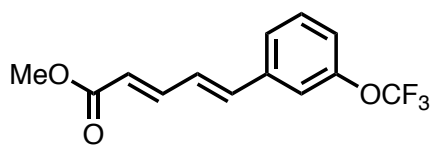

\section{Methyl (2E,4E)-5-(3-(trifluoromethoxy)phenyl)penta-2,4-dienoate (S2G) ${ }^{[6]}$}

Following general procedure, S2G was obtained as a white solid (306.6 mg, $1.13 \mathrm{mmol}, 45 \%$ yield). The spectra are in accordance with those of the compound reported in the literature. $R_{\mathrm{f}}$ value $=0.31$ (hexane/EtOAc 9:1).

\section{3) Synthesis of esters $\mathrm{S3}$}

\section{General procedure}<smiles>COC(=O)/C=C/C=C/c1cccc(Pc2ccccc2)c1</smiles> 
To a solution of $\alpha, \beta, \gamma, \delta$-unsaturated ester $\mathbf{S} 2$ in EtOAc $(0.10 \mathrm{M})$ was added palladium $(10 \%$ on carbon, $10 \mathrm{wt} \%$ ). The mixture was stirred at room temperature for several hours under $\mathrm{H}_{2}$ atmosphere with monitoring reaction progress by TLC. After the completion of the reaction, the mixture was passed through a pad of Celite ${ }^{\circledR}$ with EtOAc as eluent. The filtrate was concentrated in vacuo to afford corresponding ester $\mathbf{S 3}$. The product was used for the next step without further purification.

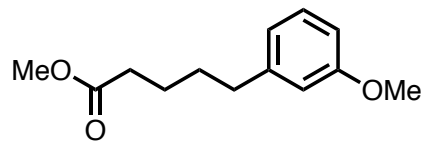

\section{Methyl 5-(3-methoxyphenyl)pentanoate (S3A)}

Following general procedure using $351.4 \mathrm{mg}$ of $\mathbf{S 2 A}$, S3A was obtained as a colorless oil $(311.8 \mathrm{mg}$, $1.40 \mathrm{mmol}, 87 \%$ yield). ${ }^{1} \mathrm{H}$ NMR $\left(400 \mathrm{MHz}, \mathrm{CDCl}_{3}\right) \delta$ 7.22-7.15 (m, 1H), 6.78-6.67 (m, 3H), 3.79 (s, $3 \mathrm{H}), 3.66(\mathrm{~s}, 3 \mathrm{H}), 2.60(\mathrm{t}, J=7.6 \mathrm{~Hz}, 2 \mathrm{H}), 2.33(\mathrm{t}, J=7.2 \mathrm{~Hz}, 2 \mathrm{H}), 1.71-1.56(\mathrm{~m}, 4 \mathrm{H}) ;{ }^{13} \mathrm{C}$ NMR $(101$ $\left.\mathrm{MHz}, \mathrm{CDCl}_{3}\right) \delta 174.2,159.7,143.9,129.4,120.9,114.3,111.1,55.2,51.6,35.7,34.0,30.9,24.7$; HRMS (ESI) $m / z$ calcd for $\mathrm{C}_{13} \mathrm{H}_{18} \mathrm{O}_{3} \mathrm{Na}[\mathrm{M}+\mathrm{Na}]^{+}: 245.1148$ found 245.1146.

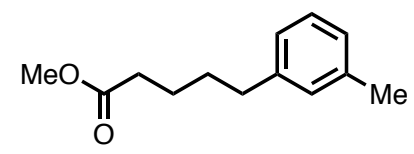

\section{Methyl 5-(m-tolyl)pentanoate (S3B)}

Following general procedure using $270.2 \mathrm{mg}$ of S2B, S3B was obtained as a colorless oil $(274.2 \mathrm{mg}$, $1.33 \mathrm{mmol}, 99 \%$ yield). ${ }^{1} \mathrm{H}$ NMR $\left(400 \mathrm{MHz}, \mathrm{CDCl}_{3}\right) \delta 7.16(\mathrm{t}, J=7.6 \mathrm{~Hz}, 1 \mathrm{H}), 7.02-6.93(\mathrm{~m}, 3 \mathrm{H})$, $3.66(\mathrm{~s}, 3 \mathrm{H}), 2.59(\mathrm{t}, J=7.2 \mathrm{~Hz}, 2 \mathrm{H}), 2.32(\mathrm{~s}, 3 \mathrm{H}), 2.36-2.26(\mathrm{~m}, 2 \mathrm{H}), 1.72-1.57(\mathrm{~m}, 4 \mathrm{H}) ;{ }^{13} \mathrm{C} \mathrm{NMR}$ $\left(101 \mathrm{MHz}, \mathrm{CDCl}_{3}\right) \delta 174.2,142.2,138.0,129.3,128.3,126.6,125.5,51.6,35.6,34.1,31.1,24.7,21.5$; HRMS (DART) $m / z$ calcd for $\mathrm{C}_{13} \mathrm{H}_{22} \mathrm{O}_{2} \mathrm{~N}\left[\mathrm{M}+\mathrm{NH}_{4}\right]^{+}: 224.1645$ found 224.1640.

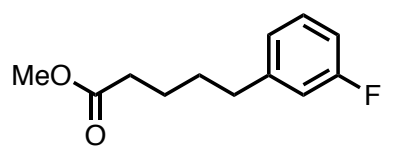

\section{Methyl 5-(3-fluorophenyl)pentanoate (S3C)}

Following general procedure using $268.1 \mathrm{mg}$ of S2C, S3C was obtained as a colorless oil $(262.7 \mathrm{mg}$, 1.25 mmol, 96\% yield). ${ }^{1} \mathrm{H}$ NMR (400 MHz, $\left.\mathrm{CDCl}_{3}\right) \delta$ 7.25-7.18 (m, $\left.1 \mathrm{H}\right), 6.94(\mathrm{~d}, J=7.2 \mathrm{~Hz}, 1 \mathrm{H})$, 6.90-6.81 (m, 2H), 3.66 (s, 3H), $2.62(\mathrm{t}, J=7.2 \mathrm{~Hz}, 2 \mathrm{H}), 2.34$ (t, $J=7.2 \mathrm{~Hz}, 2 \mathrm{H}), 1.71-1.58(\mathrm{~m}, 4 \mathrm{H})$; ${ }^{13} \mathrm{C} \mathrm{NMR}\left(101 \mathrm{MHz}, \mathrm{CDCl}_{3}\right) \delta 174.1,163.0\left(\mathrm{~d}, J_{\mathrm{C}-\mathrm{F}}=248.8 \mathrm{~Hz}\right), 144.8\left(\mathrm{~d}, J_{\mathrm{C}-\mathrm{F}}=6.8 \mathrm{~Hz}\right), 129.8(\mathrm{~d}$, $\left.J_{\mathrm{C}-\mathrm{F}}=8.7 \mathrm{~Hz}\right), 124.1,115.3\left(\mathrm{~d}, J_{\mathrm{C}-\mathrm{F}}=21.3 \mathrm{~Hz}\right), 112.8\left(\mathrm{~d}, J_{\mathrm{C}-\mathrm{F}}=21.3 \mathrm{~Hz}\right), 51.6,35.4,34.0,30.7,24.6$; HRMS (DART) $\mathrm{m} / z$ calcd for $\mathrm{C}_{12} \mathrm{H}_{19} \mathrm{O}_{2} \mathrm{NF}\left[\mathrm{M}+\mathrm{NH}_{4}\right]^{+}: 228.1394$ found 228.1389. 


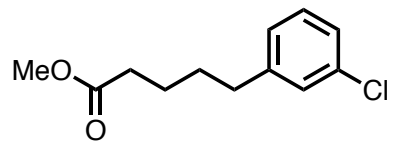

\section{Methyl 5-(3-chlorophenyl)pentanoate (S3D)}

Following general procedure using $377.9 \mathrm{mg}$ of S2D, S3D was obtained as a colorless oil $(362.4 \mathrm{mg}$, $1.60 \mathrm{mmol}, 94 \%$ yield). ${ }^{1} \mathrm{H}$ NMR $\left(400 \mathrm{MHz}, \mathrm{CDCl}_{3}\right) \delta$ 7.22-7.14 (m, 3H), $7.04(\mathrm{~d}, J=7.2 \mathrm{~Hz}, 1 \mathrm{H})$, $3.66(\mathrm{~s}, 3 \mathrm{H}), 2.60$ (t, $J=7.2 \mathrm{~Hz}, 2 \mathrm{H}), 2.33(\mathrm{t}, J=7.2 \mathrm{~Hz}, 2 \mathrm{H}), 1.71-1.58(\mathrm{~m}, 4 \mathrm{H}) ;{ }^{13} \mathrm{C} \mathrm{NMR}(101 \mathrm{MHz}$, $\left.\mathrm{CDCl}_{3}\right) \delta 174.1,144.2,134.2,129.7,128.6,126.7,126.1,51.6,35.3,34.0,30.7,24.6$; HRMS (DART) $m / z$ calcd for $\mathrm{C}_{12} \mathrm{H}_{19} \mathrm{O}_{2} \mathrm{NCl}\left[\mathrm{M}+\mathrm{NH}_{4}\right]^{+}: 244.1099$ found 244.1095 .

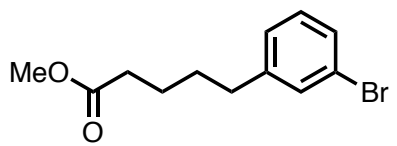

\section{Methyl 5-(3-bromophenyl)pentanoate (S3E)}

Following general procedure using $318.3 \mathrm{mg}$ of $\mathbf{S 2 E}, \mathbf{S 3 E}$ was obtained as a colorless oil $(260.2 \mathrm{mg}$, $0.960 \mathrm{mmol}, 81 \%$ yield). ${ }^{1} \mathrm{H}$ NMR $\left(400 \mathrm{MHz}, \mathrm{CDCl}_{3}\right) \delta$ 7.33-7.27 (m, 2H), 7.18-7.06 (m, 2H), 3.67 (s, $3 \mathrm{H}), 2.60(\mathrm{t}, J=7.2 \mathrm{~Hz}, 2 \mathrm{H}), 2.34(\mathrm{t}, J=7.2 \mathrm{~Hz}, 2 \mathrm{H}), 1.71-1.58(\mathrm{~m}, 4 \mathrm{H}) ;{ }^{13} \mathrm{C}$ NMR $(101 \mathrm{MHz}$, $\left.\mathrm{CDCl}_{3}\right) \delta 174.1,144.6,131.5,130.0,129.0,127.2,122.5,51.7,35.3,34.0,30.7,24.6$; HRMS (DART) $m / z$ calcd for $\mathrm{C}_{12} \mathrm{H}_{19} \mathrm{O}_{2} \mathrm{NBr}\left[\mathrm{M}+\mathrm{NH}_{4}\right]^{+}: 288.0594$ found 288.0588 .

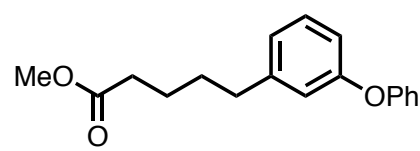

\section{Methyl 5-(3-phenoxyphenyl)pentanoate (S3F)}

Following general procedure using $369.8 \mathrm{mg}$ of $\mathbf{S 2 F}$, S3F was obtained as a colorless oil $(357.5 \mathrm{mg}$, $1.26 \mathrm{mmol}, 95 \%$ yield). ${ }^{1} \mathrm{H}$ NMR (400 MHz, $\left.\mathrm{CDCl}_{3}\right) \delta 7.32(\mathrm{~m}, 2 \mathrm{H}), 7.22(\mathrm{t}, J=8.0 \mathrm{~Hz}, 1 \mathrm{H}), 7.08(\mathrm{t}$, $J=7.6 \mathrm{~Hz}, 1 \mathrm{H}), 7.00$ (d, $J=7.6 \mathrm{~Hz}, 2 \mathrm{H}), 6.91(\mathrm{~d}, J=8.0 \mathrm{~Hz}, 1 \mathrm{H}), 6.86-6.79$ (m, 2H), 3.65 (s, 3H), $2.60(\mathrm{t}, J=7.2 \mathrm{~Hz}, 2 \mathrm{H}), 2.32(\mathrm{t}, J=7.2 \mathrm{~Hz}, 2 \mathrm{H}), 1.71-1.58(\mathrm{~m}, 4 \mathrm{H}) ;{ }^{13} \mathrm{C} \mathrm{NMR}\left(101 \mathrm{MHz}, \mathrm{CDCl}_{3}\right) \delta$ 174.1, 157.4, 157.3, 144.3, 129.8, 129.6, 123.5, 123.2, 119.0, 118.9, 116.4, 51.6, 35.5, 34.0, 30.8, 24.6; HRMS (DART) $m / z$ calcd for $\mathrm{C}_{18} \mathrm{H}_{24} \mathrm{O}_{3} \mathrm{~N}\left[\mathrm{M}+\mathrm{NH}_{4}\right]^{+}: 302.1751$ found 302.1744 .

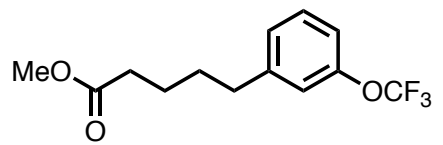

\section{Methyl 5-(3-(trifluoromethoxy)phenyl)pentanoate (S3G)}

Following general procedure using $256.3 \mathrm{mg}$ of S2G, S3G was obtained as a colorless oil (246.1 mg, $0.891 \mathrm{mmol}, 95 \%$ yield). ${ }^{1} \mathrm{H}$ NMR $\left(400 \mathrm{MHz}, \mathrm{CDCl}_{3}\right) \delta 7.28$ (t, $\left.J=7.6 \mathrm{~Hz}, 1 \mathrm{H}\right), 7.10(\mathrm{~d}, J=7.6$ $\mathrm{Hz}, 1 \mathrm{H}), 7.05-7.02(\mathrm{~m}, 2 \mathrm{H}), 3.66(\mathrm{~s}, 3 \mathrm{H}), 2.65$ (t, $J=6.8 \mathrm{~Hz}, 2 \mathrm{H}), 2.34(\mathrm{t}, J=6.8 \mathrm{~Hz}, 2 \mathrm{H}), 1.72-1.60$ 
$(\mathrm{m}, 4 \mathrm{H}) ;{ }^{13} \mathrm{C}$ NMR $\left(101 \mathrm{MHz}, \mathrm{CDCl}_{3}\right) \delta 174.0,149.4,144.5,129.7,126.9,121.0,120.6$ (q, $J_{\mathrm{C}-\mathrm{F}}=$ $260.3 \mathrm{~Hz}$ ), 118.4, 51.6, 35.4, 33.9, 30.7, 24.6; HRMS (DART) $\mathrm{m} / z$ calcd for $\mathrm{C}_{13} \mathrm{H}_{19} \mathrm{O}_{3} \mathrm{NF}_{3}\left[\mathrm{M}+\mathrm{NH}_{4}\right]^{+}$: 294.1312 found 294.1304. 


\section{4) Synthesis of esters S3I-L}

\section{General procedure}

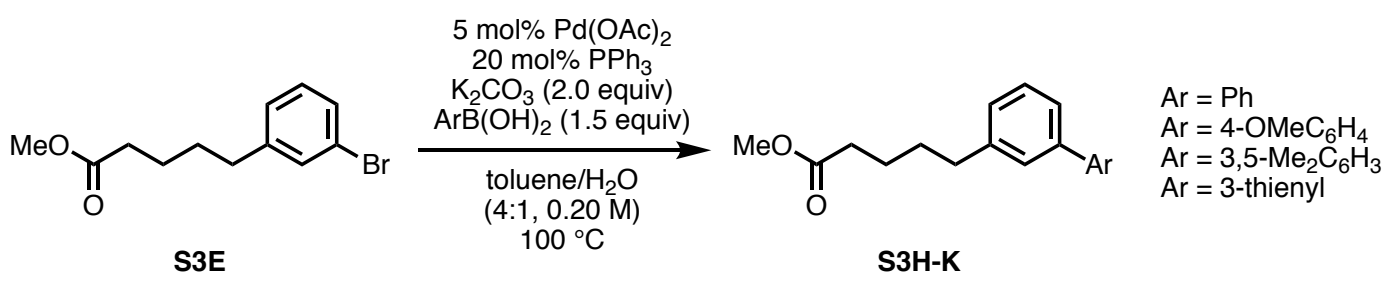

To a 25-mL glass vessel containing a magnetic stirring bar were added bromide S3E (271.2 mg, 1.0 mmol, 1.0 equiv), aryl boronic acid ( $1.5 \mathrm{mmol}, 1.5$ equiv), potassium carbonate ( $276.4 \mathrm{mg}, 2.0 \mathrm{mmol}$, 2.0 equiv), palladium acetate $(11.2 \mathrm{mg}, 0.050 \mathrm{mmol}, 5 \mathrm{~mol} \%)$, triphenyl phosphine $(52.5 \mathrm{mg}, 0.20$ mmol, $20 \mathrm{~mol} \%)$, toluene $(4 \mathrm{~mL})$, and $\mathrm{H}_{2} \mathrm{O}(1 \mathrm{~mL})$. The vessel was sealed with a screw cap and then heated at $100{ }^{\circ} \mathrm{C}$ with stirring overnight. After cooling the reaction mixture to room temperature, the mixture was passed through a short silica gel pad with EtOAc as eluent and then concentrated in vacuo. The residue was purified by Isolera ${ }^{\circledR}$ (hexane/EtOAc 95:5 to 90:10) to afford corresponding ester $\mathbf{S 3}$.

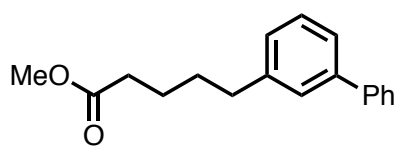

\section{Methyl 5-([1,1'-biphenyl]-3-yl)pentanoate (S3H)}

Following general procedure, $\mathbf{S 3 H}$ was obtained as a colorless oil $(248.8 \mathrm{mg}, 0.927 \mathrm{mmol}, 93 \%$ yield). ${ }^{1} \mathrm{H}$ NMR (400 MHz, $\left.\mathrm{CDCl}_{3}\right) \delta 7.58(\mathrm{~d}, J=8.0 \mathrm{~Hz}, 2 \mathrm{H}), 7.48-7.38(\mathrm{~m}, 4 \mathrm{H}), 7.38-7.31(\mathrm{~m}, 2 \mathrm{H})$, $7.16(\mathrm{~d}, J=8.0 \mathrm{~Hz}, 1 \mathrm{H}), 3.65(\mathrm{~s}, 3 \mathrm{H}), 2.69$ (t, $J=7.2 \mathrm{~Hz}, 2 \mathrm{H}), 2.34$ (t, $J=6.8 \mathrm{~Hz}, 2 \mathrm{H}), 1.76-1.63$ (m, $4 \mathrm{H}) ;{ }^{13} \mathrm{C}$ NMR $\left(101 \mathrm{MHz}, \mathrm{CDCl}_{3}\right) \delta 174.2,142.7,141.5,141.4,128.9,128.8,127.5,127.4,127.3$, 124.8, 51.6, 35.8, 34.1, 31.1, 24.7 (one peak is missing due to overlapping); HRMS (ESI) $\mathrm{m} / \mathrm{z}$ calcd for $\mathrm{C}_{18} \mathrm{H}_{20} \mathrm{O}_{2} \mathrm{Na}[\mathrm{M}+\mathrm{Na}]^{+}: 291.1356$ found 291.1351; $\mathrm{R}_{\mathrm{f}}$ value $=0.43$ (hexane/EtOAc 9:1).

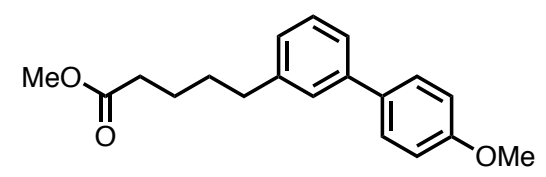

\section{Methyl 5-(4'-methoxy-[1,1'-biphenyl]-3-yl)pentanoate (S3I)}

Following general procedure, S3I was obtained as a colorless oil $(263.0 \mathrm{mg}, 0.881 \mathrm{mmol}, 88 \%$ yield). ${ }^{1} \mathrm{H}$ NMR (400 MHz, $\left.\mathrm{CDCl}_{3}\right) \delta 7.51(\mathrm{~d}, J=8.4 \mathrm{~Hz}, 2 \mathrm{H}), 7.39-7.27(\mathrm{~m}, 3 \mathrm{H}), 7.11(\mathrm{~d}, J=7.2 \mathrm{~Hz}$, $1 \mathrm{H}), 6.96(\mathrm{~d}, J=8.4 \mathrm{~Hz}, 2 \mathrm{H}), 3.84(\mathrm{~s}, 3 \mathrm{H}), 3.65(\mathrm{~s}, 3 \mathrm{H}), 2.67$ (t, $J=7.2 \mathrm{~Hz}, 2 \mathrm{H}), 2.34(\mathrm{t}, J=6.8 \mathrm{~Hz}$, 2H), 1.75-1.65 (m, 4H); ${ }^{13} \mathrm{C}$ NMR (101 MHz, $\left.\mathrm{CDCl}_{3}\right) \delta 174.2,159.2,142.7,141.0,134.0,128.8$, 128.3, 127.0, 126.9, 124.4, 114.3, 55.4, 51.6, 35.8, 34.1, 31.1, 24.7; HRMS (ESI) $\mathrm{m} / \mathrm{z}$ calcd for $\mathrm{C}_{19} \mathrm{H}_{22} \mathrm{O}_{3} \mathrm{Na}[\mathrm{M}+\mathrm{Na}]^{+}: 321.1461$ found 321.1458; $\mathrm{R}_{\mathrm{f}}$ value $=0.27$ (hexane/EtOAc 9:1). 


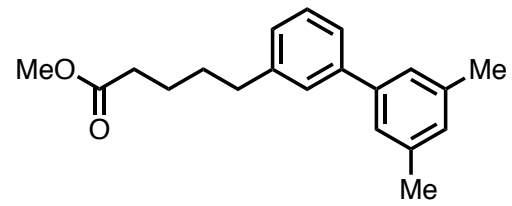

\section{Methyl 5-(3',5'-dimethyl-[1,1'-biphenyl]-3-yl)pentanoate (S3J)}

Following general procedure, S3J was obtained as a colorless oil $(281.6 \mathrm{mg}, 0.950 \mathrm{mmol}, 95 \%$ yield). ${ }^{1} \mathrm{H}$ NMR $\left(400 \mathrm{MHz}, \mathrm{CDCl}_{3}\right) \delta$ 7.42-7.36 (m, 2H), $7.32(\mathrm{t}, J=7.6 \mathrm{~Hz}, 1 \mathrm{H}), 7.20(\mathrm{~s}, 2 \mathrm{H}), 7.14$ (d, $J=8.0 \mathrm{~Hz}, 1 \mathrm{H}), 6.99(\mathrm{~s}, 1 \mathrm{H}), 3.66(\mathrm{~s}, 3 \mathrm{H}), 2.69$ (t, $J=7.2 \mathrm{~Hz}, 2 \mathrm{H}), 2.38(\mathrm{~s}, 6 \mathrm{H}), 2.41-2.29(\mathrm{~m}, 2 \mathrm{H})$, 1.75-1.63 (m, 4H); ${ }^{13} \mathrm{C}$ NMR $\left(101 \mathrm{MHz}, \mathrm{CDCl}_{3}\right) \delta 174.2,142.6,141.6,141.5,138.3,129.0,128.7$, 127.4, 127.3, 125.2, 124.9, 51.6, 35.8, 34.1, 31.1, 24.8, 21.5; HRMS (ESI) $\mathrm{m} / z$ calcd for $\mathrm{C}_{20} \mathrm{H}_{24} \mathrm{O}_{2} \mathrm{Na}$ $[\mathrm{M}+\mathrm{Na}]^{+}: 319.1669$ found $319.1665 ; \mathrm{R}_{\mathrm{f}}$ value $=0.45$ (hexane/EtOAc 9:1).

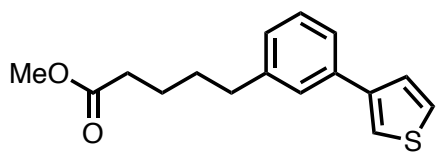

\section{Methyl 5-(3-(thiophen-3-yl)phenyl)pentanoate (S3K)}

Following general procedure, S3K was obtained as a colorless oil $(163.9 \mathrm{mg}, 0.597 \mathrm{mmol}, 60 \%$ yield). ${ }^{1} \mathrm{H}$ NMR (400 MHz, $\left.\mathrm{CDCl}_{3}\right) \delta$ 7.46-7.34 (m, 5H), $7.31(\mathrm{t}, J=7.6 \mathrm{~Hz}, 1 \mathrm{H}), 7.11(\mathrm{~d}, J=7.6 \mathrm{~Hz}$, 1H), 3.66 (s, 3H), 2.67 (t, $J=7.2 \mathrm{~Hz}, 2 \mathrm{H}), 2.35$ (t, $J=6.8 \mathrm{~Hz}, 2 \mathrm{H}), 1.74-1.63(\mathrm{~m}, 4 \mathrm{H}) ;{ }^{13} \mathrm{C}$ NMR (101 $\left.\mathrm{MHz}, \mathrm{CDCl}_{3}\right) \delta 174.2,142.8,142.6,136.0,128.9,127.4,126.7,126.5,126.2,124.1,120.3,51.6,35.7$, 34.0, 31.0, 24.7; HRMS (ESI) $m / z$ calcd for $\mathrm{C}_{16} \mathrm{H}_{18} \mathrm{O}_{2} \mathrm{NaS}[\mathrm{M}+\mathrm{Na}]^{+}: 297.0920$ found 297.0917; $\mathrm{R}_{\mathrm{f}}$ value $=0.38$ (hexane/EtOAc 9:1)

\section{5) Synthesis of $\alpha$-diazoester $7^{[4]}$}

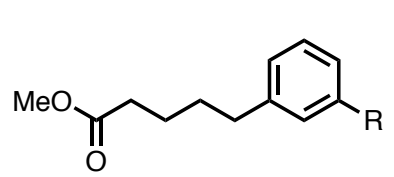

S3

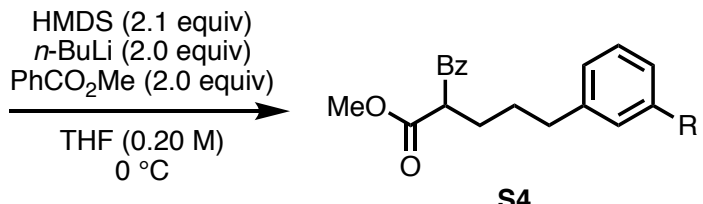

S4

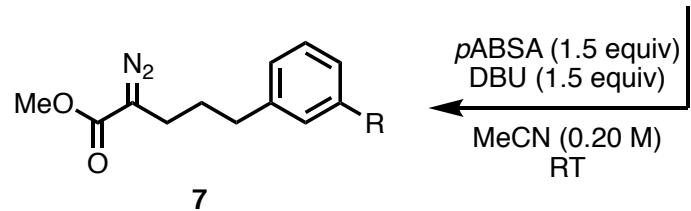

A 50-mL round bottom flask containing a magnetic stirring bar was dried with a heat-gun in vacuo and filled with $\mathrm{N}_{2}$ after cooling to room temperature. To this flask were added anhydrous THF ( 0.40 M) and bis(trimethylsilyl)amine (HMDS, 2.1 equiv). To this solution was slowly added $n$-BuLi (1.55 $\mathrm{M}$ in hexane, 2.0 equiv) at $0{ }^{\circ} \mathrm{C}$. After stirring for 10 minutes, methyl benzoate (2.0 equiv) and ester S3 $\left(0.40 \mathrm{M}\right.$ in anydrous THF) were added at $0{ }^{\circ} \mathrm{C}$. The mixture was stirred for 10 minutes and then poured into sat. $\mathrm{NH}_{4} \mathrm{Cl}$ aq. The resulting mixture was extracted two times with EtOAc, dried over 
$\mathrm{Na}_{2} \mathrm{SO}_{4}$, filtrated and concentrated in vacuo. The product $\mathbf{S} 4$ was used for the next step without further purification.

To a solution of $\mathbf{S} 4$ in $\mathrm{MeCN}(0.20 \mathrm{M})$ were added $p$-acetamidobenzenesulfonyl azide ( $p$ ABSA, 1.5 equiv) and 1,8-diazabicyclo[5.4.0]undec-7-ene (DBU, 1.5 equiv) at $0{ }^{\circ} \mathrm{C}$. After stirring the mixture for 3 hours at room temperature, the reaction was quenched with water. The mixture was extracted two times with diethylether, dried over $\mathrm{Na}_{2} \mathrm{SO}_{4}$, filtrated, and concentrated in vacuo. The residue was purified by Isolera ${ }^{\circledR}$ (hexane/EtOAc 95:5 to 90:10) to afford corresponding $\alpha$-diazoester 7 .

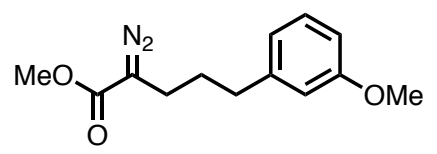

\section{Methyl 2-diazo-5-(3-methoxyphenyl)pentanoate (7A)}

Following general procedure using $265.5 \mathrm{mg}$ of S3A, 7A was obtained as a yellow oil (236.8 mg, $0.954 \mathrm{mmol}, 80 \%$ yield in 2 steps). ${ }^{1} \mathrm{H}$ NMR $\left(400 \mathrm{MHz}, \mathrm{CDCl}_{3}\right) \delta$ 7.23-7.18 (m, $\left.1 \mathrm{H}\right), 6.79-6.70(\mathrm{~m}$, $3 \mathrm{H}), 3.80(\mathrm{~s}, 3 \mathrm{H}), 3.76(\mathrm{~s}, 3 \mathrm{H}), 2.66(\mathrm{t}, J=7.6 \mathrm{~Hz}, 2 \mathrm{H}), 2.34(\mathrm{t}, J=7.6 \mathrm{~Hz}, 2 \mathrm{H}), 1.85$ (quintet, $J=7.6$ $\mathrm{Hz}, 2 \mathrm{H}) ;{ }^{13} \mathrm{C}$ NMR $\left(101 \mathrm{MHz}, \mathrm{CDCl}_{3}\right) \delta 159.8,143.0,129.5,120.8,114.2,111.4,55.2,52.0,35.0$, 29.2, $22.8\left(\mathrm{C}=\mathrm{N}_{2}\right.$ and $\mathrm{C}=\mathrm{O}$ carbons were not observed); HRMS (ESI) $\mathrm{m} / z$ calcd for $\mathrm{C}_{13} \mathrm{H}_{16} \mathrm{O}_{3} \mathrm{~N}_{2} \mathrm{Na}$ $[\mathrm{M}+\mathrm{Na}]^{+}: 271.1053$ found 271.1049; $\mathrm{R}_{\mathrm{f}}$ value $=0.42$ (hexane/EtOAc 4:1).

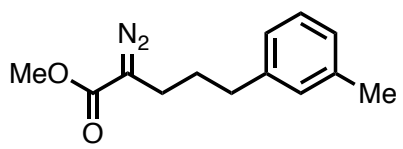

\section{Methyl 2-diazo-5-(m-tolyl)pentanoate (7B)}

Following general procedure using $253.0 \mathrm{mg}$ of S3B, 7B was obtained as a yellow oil (185.4 mg, $0.798 \mathrm{mmol}, 65 \%$ yield in 2 steps). ${ }^{1} \mathrm{H}$ NMR $\left(400 \mathrm{MHz}, \mathrm{CDCl}_{3}\right) \delta 7.17$ (t, $\left.J=7.2 \mathrm{~Hz}, 1 \mathrm{H}\right), 7.03-6.95$ $(\mathrm{m}, 3 \mathrm{H}), 3.76(\mathrm{~s}, 3 \mathrm{H}), 2.64(\mathrm{t}, J=8.0 \mathrm{~Hz}, 2 \mathrm{H}), 2.37-2.28(\mathrm{~m}, 2 \mathrm{H}), 2.33(\mathrm{~s}, 3 \mathrm{H}), 1.83$ (quintet, $J=8.0$ $\mathrm{Hz}, 2 \mathrm{H}) ;{ }^{13} \mathrm{C} \mathrm{NMR}\left(101 \mathrm{MHz}, \mathrm{CDCl}_{3}\right) \delta 141.4,138.1,129.3,128.4,126.9,125.5,52.0,34.9,29.4$, 22.9, $21.5\left(\mathrm{C}=\mathrm{N}_{2}\right.$ and $\mathrm{C}=\mathrm{O}$ carbons were not observed); HRMS (DART) $\mathrm{m} / z$ calcd for $\mathrm{C}_{13} \mathrm{H}_{20} \mathrm{O}_{2} \mathrm{~N}_{3}$ $\left[\mathrm{M}+\mathrm{NH}_{4}\right]^{+}: 250.1550$ found $250.1544 ; \mathrm{R}_{\mathrm{f}}$ value $=0.41($ hexane/EtOAc 9:1).

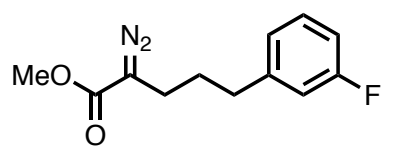

\section{Methyl 2-diazo-5-(3-fluorophenyl)pentanoate (7C)}

Following general procedure using $236.9 \mathrm{mg}$ of S3C, 7C was obtained as a yellow oil (202.8 mg, $0.858 \mathrm{mmol}, 76 \%$ yield in 2 steps). ${ }^{1} \mathrm{H}$ NMR $\left(400 \mathrm{MHz}, \mathrm{CDCl}_{3}\right) \delta$ 7.25-7.20 (m, 1H), 6.95 (d, $J=7.6$ $\mathrm{Hz}, 1 \mathrm{H}), 6.91-6.85(\mathrm{~m}, 2 \mathrm{H}), 3.76(\mathrm{~s}, 3 \mathrm{H}), 2.67(\mathrm{t}, J=7.6 \mathrm{~Hz}, 2 \mathrm{H}), 2.34(\mathrm{t}, J=7.6 \mathrm{~Hz}, 2 \mathrm{H}), 1.84$ (quintet, $J=7.6 \mathrm{~Hz}, 2 \mathrm{H}) ;{ }^{13} \mathrm{C} \mathrm{NMR}\left(101 \mathrm{MHz}, \mathrm{CDCl}_{3}\right) \delta 168.0,163.0\left(\mathrm{~d}, J_{\mathrm{C}-\mathrm{F}}=248.7 \mathrm{~Hz}\right), 144.0(\mathrm{~d}$, $\left.J_{\mathrm{C}-\mathrm{F}}=7.7 \mathrm{~Hz}\right), 129.9\left(\mathrm{~d}, J_{\mathrm{C}-\mathrm{F}}=7.8 \mathrm{~Hz}\right), 124.1\left(\mathrm{~d}, J_{\mathrm{C}-\mathrm{F}}=1.9 \mathrm{~Hz}\right), 115.3\left(\mathrm{~d}, J_{\mathrm{C}-\mathrm{F}}=21.2 \mathrm{~Hz}\right), 113.0(\mathrm{~d}$, 
$J_{\mathrm{C}-\mathrm{F}}=21.3 \mathrm{~Hz}$ ), 52.0, 34.7, 29.1, $22.8\left(\mathrm{C}=\mathrm{N}_{2}\right.$ carbon was not observed); HRMS (DART) $\mathrm{m} / \mathrm{z}$ calcd for $\mathrm{C}_{12} \mathrm{H}_{17} \mathrm{O}_{2} \mathrm{~N}_{3} \mathrm{~F}\left[\mathrm{M}+\mathrm{NH}_{4}\right]^{+}: 254.1299$ found 254.1293; $\mathrm{R}_{\mathrm{f}}$ value = 0.31 (hexane/EtOAc 9:1).

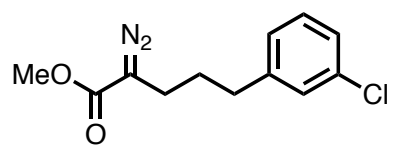

\section{Methyl 5-(3-chlorophenyl)-2-diazopentanoate (7D)}

Following general procedure using $349.2 \mathrm{mg}$ of S3D, 7D was obtained as a yellow oil $(257.1 \mathrm{mg}$, $1.02 \mathrm{mmol}, 66 \%$ yield in 2 steps). ${ }^{1} \mathrm{H}$ NMR $\left(400 \mathrm{MHz}, \mathrm{CDCl}_{3}\right) \delta 7.23-7.14(\mathrm{~m}, 3 \mathrm{H}), 7.05$ (d, $J=7.2$ $\mathrm{Hz}, 1 \mathrm{H}), 3.76(\mathrm{~s}, 3 \mathrm{H}), 2.65$ (t, $J=7.6 \mathrm{~Hz}, 2 \mathrm{H}), 2.34$ (t, $J=7.6 \mathrm{~Hz}, 2 \mathrm{H}), 1.83$ (quintet, $J=7.6 \mathrm{~Hz}, 2 \mathrm{H}$ ); ${ }^{13} \mathrm{C}$ NMR $\left(101 \mathrm{MHz}, \mathrm{CDCl}_{3}\right) \delta 167.9,143.4,134.2,129.7,128.5,126.6,126.3,52.0,34.6,29.1,22.8$ $\left(\mathrm{C}=\mathrm{N}_{2}\right.$ carbon was not observed); HRMS (DART) $\mathrm{m} / z$ calcd for $\mathrm{C}_{12} \mathrm{H}_{17} \mathrm{O}_{2} \mathrm{~N}_{3} \mathrm{Cl}\left[\mathrm{M}+\mathrm{NH}_{4}\right]^{+}: 270.1004$ found 270.0997; $\mathrm{R}_{\mathrm{f}}$ value $=0.28$ (hexane/EtOAc 9:1)

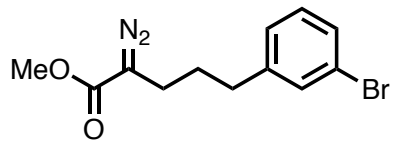

\section{Methyl 5-(3-bromophenyl)-2-diazopentanoate (7E)}

Following general procedure using $260.2 \mathrm{mg}$ of S3E, 7E was obtained as a yellow oil (149.9 mg, $0.504 \mathrm{mmol}, 53 \%$ yield in 2 steps). ${ }^{1} \mathrm{H}$ NMR (400 MHz, $\left.\mathrm{CDCl}_{3}\right) \delta$ 7.35-7.27 (m, 2H), 7.17-7.08 (m, $2 \mathrm{H}$ ), $3.76(\mathrm{~s}, 3 \mathrm{H}), 2.64$ (t, $J=7.6 \mathrm{~Hz}, 2 \mathrm{H}), 2.34$ (t, $J=7.6 \mathrm{~Hz}, 2 \mathrm{H}), 1.83$ (quintet, $J=7.6 \mathrm{~Hz}, 2 \mathrm{H}$ ); ${ }^{13} \mathrm{C}$ NMR $\left(101 \mathrm{MHz}, \mathrm{CDCl}_{3}\right) \delta 143.8,131.5,130.1,129.3,127.1,122.6,52.0,34.6,29.2,22.9\left(\mathrm{C}=\mathrm{N}_{2}\right.$ and $\mathrm{C}=\mathrm{O}$ carbons were not observed); HRMS (DART) $\mathrm{m} / z$ calcd for $\mathrm{C}_{12} \mathrm{H}_{17} \mathrm{O}_{2} \mathrm{~N}_{3} \mathrm{Br}\left[\mathrm{M}+\mathrm{NH}_{4}\right]^{+}: 314.0499$ found 314.0492; $\mathrm{R}_{\mathrm{f}}$ value $=0.35$ (hexane/EtOAc 9:1).

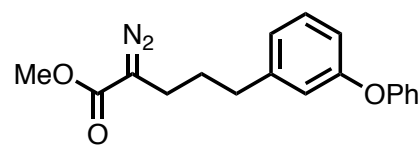

\section{Methyl 2-diazo-5-(3-phenoxyphenyl)pentanoate (7F)}

Following general procedure using $322.9 \mathrm{mg}$ of S3F, 7F was obtained as a yellow oil $(246.0 \mathrm{mg}$, $0.793 \mathrm{mmol}, 70 \%$ yield in 2 steps). ${ }^{1} \mathrm{H}$ NMR (400 MHz, $\left.\mathrm{CDCl}_{3}\right) \delta 7.33(\mathrm{~m}, 2 \mathrm{H}), 7.27-7.21(\mathrm{~m}, 1 \mathrm{H})$, $7.10(\mathrm{t}, J=7.2 \mathrm{~Hz}, 1 \mathrm{H}), 7.00(\mathrm{~m}, 2 \mathrm{H}), 6.92$ (d, $J=7.6 \mathrm{~Hz}, 1 \mathrm{H}), 6.85-6.80(\mathrm{~m}, 2 \mathrm{H}), 3.76(\mathrm{~s}, 3 \mathrm{H}), 2.65$ (t, $J=7.6 \mathrm{~Hz}, 2 \mathrm{H}), 2.34$ (t, $J=7.6 \mathrm{~Hz}, 2 \mathrm{H}), 1.83$ (quintet, $J=7.6 \mathrm{~Hz}, 2 \mathrm{H}) ;{ }^{13} \mathrm{C}$ NMR $(101 \mathrm{MHz}$, $\left.\mathrm{CDCl}_{3}\right) \delta 167.9,157.4,157.3,143.5,129.8,129.7,123.3,123.2,118.9,116.5,51.9,34.8,29.1,22.8$ (one peak is missing due to overlapping, $\mathrm{C}=\mathrm{N}_{2}$ carbon was not observed); HRMS (ESI) $\mathrm{m} / \mathrm{z}$ calcd for $\mathrm{C}_{18} \mathrm{H}_{18} \mathrm{O}_{3} \mathrm{~N}_{2} \mathrm{Na}[\mathrm{M}+\mathrm{Na}]^{+}: 333.1210$ found 333.1205; $\mathrm{R}_{\mathrm{f}}$ value $=0.30$ (hexane/EtOAc 9:1). 


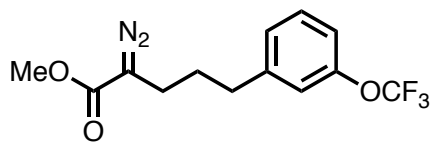

\section{Methyl 2-diazo-5-(3-(trifluoromethoxy)phenyl)pentanoate (7G)}

Following general procedure using $221.1 \mathrm{mg}$ of S3G, 7G was obtained as a yellow oil (152.9 mg, $0.506 \mathrm{mmol}, 63 \%$ yield in 2 steps). ${ }^{1} \mathrm{H}$ NMR $\left(400 \mathrm{MHz}, \mathrm{CDCl}_{3}\right) \delta 7.30(\mathrm{t}, J=8.0 \mathrm{~Hz}, 1 \mathrm{H}), 7.11(\mathrm{~d}, J=$ $8.0 \mathrm{~Hz}, 1 \mathrm{H}), 7.06-7.02(\mathrm{~m}, 2 \mathrm{H}), 3.76(\mathrm{~s}, 3 \mathrm{H}), 2.69(\mathrm{t}, J=7.6 \mathrm{~Hz}, 2 \mathrm{H}), 2.35$ (t, $J=7.6 \mathrm{~Hz}, 2 \mathrm{H}), 1.86$ (quintet, $J=7.6 \mathrm{~Hz}, 2 \mathrm{H}) ;{ }^{13} \mathrm{C} \mathrm{NMR}\left(101 \mathrm{MHz}, \mathrm{CDCl}_{3}\right) \delta 167.9,149.5,143.8,129.8,126.9,120.9$, $120.6\left(\mathrm{q}, J_{\mathrm{C}-\mathrm{F}}=261.3 \mathrm{~Hz}\right), 118.6,52.0,34.7,29.1,22.9\left(\mathrm{C}=\mathrm{N}_{2}\right.$ carbon was not observed $)$; HRMS (ESI) $\mathrm{m} / z$ calcd for $\mathrm{C}_{13} \mathrm{H}_{13} \mathrm{O}_{3} \mathrm{~N}_{2} \mathrm{~F}_{3} \mathrm{Na}[\mathrm{M}+\mathrm{Na}]^{+}: 325.0770$ found $325.0765 ; \mathrm{R}_{\mathrm{f}}$ value $=0.39$ (hexane/EtOAc 9:1).

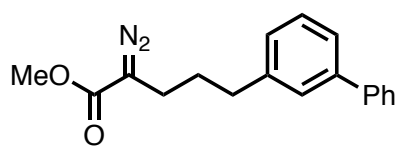

\section{Methyl 5-([1,1'-biphenyl]-3-yl)-2-diazopentanoate (7H)}

Following general procedure using $248.8 \mathrm{mg}$ of $\mathbf{S 3 H}, \mathbf{7 H}$ was obtained as a yellow oil $(188.1 \mathrm{mg}$, $0.639 \mathrm{mmol}, 69 \%$ yield in 2 steps). ${ }^{1} \mathrm{H}$ NMR $\left(400 \mathrm{MHz}, \mathrm{CDCl}_{3}\right) \delta 7.59(\mathrm{~d}, J=7.2 \mathrm{~Hz}, 2 \mathrm{H}), 7.49-7.38$ (m, 4H), 7.38-7.32 (m, 2H), 7.17 (d, $J=7.2 \mathrm{~Hz}, 1 \mathrm{H}), 3.76(\mathrm{~s}, 3 \mathrm{H}), 2.75(\mathrm{t}, J=7.6 \mathrm{~Hz}, 2 \mathrm{H}), 2.38$ (t, $J=$ $7.6 \mathrm{~Hz}, 2 \mathrm{H}$ ), 1.90 (quintet, $J=7.6 \mathrm{~Hz}, 2 \mathrm{H}) ;{ }^{13} \mathrm{C} \mathrm{NMR}\left(101 \mathrm{MHz}, \mathrm{CDCl}_{3}\right) \delta 141.9,141.5,141.3,129.0$, $128.8,127.40,127.36,127.3,125.1,52.0,35.1,29.4,22.9$ (one peak is missing due to overlapping, $\mathrm{C}=\mathrm{N}_{2}$ and $\mathrm{C}=\mathrm{O}$ carbons were not observed); HRMS (ESI) $m / z$ calcd for $\mathrm{C}_{18} \mathrm{H}_{18} \mathrm{O}_{2} \mathrm{~N}_{2} \mathrm{Na}[\mathrm{M}+\mathrm{Na}]^{+}$: 317.1260 found 317.1258; $R_{\mathrm{f}}$ value $=0.42$ (hexane/EtOAc 9:1).

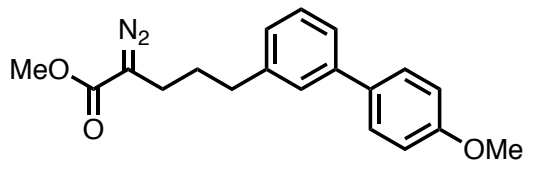

\section{Methyl 2-diazo-5-(4'-methoxy-[1,1'-biphenyl]-3-yl)pentanoate (7I)}

Following general procedure using $263.0 \mathrm{mg}$ of S3I, 7I was obtained as a yellow oil $(178.1 \mathrm{mg}$, $0.549 \mathrm{mmol}, 62 \%$ yield in 2 steps). ${ }^{1} \mathrm{H}$ NMR $\left(400 \mathrm{MHz}, \mathrm{CDCl}_{3}\right) \delta 7.52(\mathrm{~d}, J=8.4 \mathrm{~Hz}, 2 \mathrm{H}), 7.41-7.30$ (m, 3H), $7.13(\mathrm{~d}, J=7.2 \mathrm{~Hz}, 1 \mathrm{H}), 6.97(\mathrm{~d}, J=8.4 \mathrm{~Hz}, 2 \mathrm{H}), 3.85$ (s, 3H), 3.76 (s, 3H), 2.73 (t, $J=7.6$ $\mathrm{Hz}, 2 \mathrm{H}$ ), 2.38 (t, $J=7.6 \mathrm{~Hz}, 2 \mathrm{H}$ ), 1.89 (quintet, $J=7.6 \mathrm{~Hz}, 2 \mathrm{H}$ ); ${ }^{13} \mathrm{C} \mathrm{NMR}\left(101 \mathrm{MHz}, \mathrm{CDCl}_{3}\right.$ ) $\delta 159.2$, $141.9,141.1,133.9,128.9,128.3,127.0,126.8,124.6,114.3,55.4,52.0,35.1,29.4,22.9\left(\mathrm{C}=\mathrm{N}_{2}\right.$ and $\mathrm{C}=\mathrm{O}$ carbons were not observed); HRMS (ESI) $\mathrm{m} / z$ calcd for $\mathrm{C}_{19} \mathrm{H}_{20} \mathrm{O}_{3} \mathrm{~N}_{2} \mathrm{Na}[\mathrm{M}+\mathrm{Na}]^{+}: 347.1366$ found 347.1363; $\mathrm{R}_{\mathrm{f}}$ value $=0.31$ (hexane/EtOAc 9:1). 
<smiles>COC(=O)C(=N)CCCc1cccc(-c2cc([N+](=O)[O-])cc([N+](=O)[O-])c2)c1</smiles>

\section{Methyl 2-diazo-5-(3',5'-dimethyl-[1,1'-biphenyl]-3-yl)pentanoate (7J)}

Following general procedure using $281.6 \mathrm{mg}$ of S3J, 7J was obtained as a yellow oil (176.7 $\mathrm{mg}$, $0.548 \mathrm{mmol}, 58 \%$ yield in 2 steps). ${ }^{1} \mathrm{H}$ NMR $\left(400 \mathrm{MHz}, \mathrm{CDCl}_{3}\right) \delta 7.42-7.36(\mathrm{~m}, 2 \mathrm{H}), 7.33(\mathrm{t}, J=7.6$ $\mathrm{Hz}, 1 \mathrm{H}), 7.20$ (s, 2H), 7.14 (d, $J=7.6 \mathrm{~Hz}, 1 \mathrm{H}), 6.99$ (s, 1H), 3.76 (s, 3H), 2.74 (t, $J=7.6 \mathrm{~Hz}, 2 \mathrm{H})$, 2.45-2.32 (m, 2H), $2.38(\mathrm{~s}, 6 \mathrm{H}), 1.89$ (quintet, $J=7.6 \mathrm{~Hz}, 2 \mathrm{H}) ;{ }^{13} \mathrm{C} \mathrm{NMR}\left(101 \mathrm{MHz}, \mathrm{CDCl}_{3}\right) \delta 141.79$, $141.76,141.4,138.4,129.0,128.9,127.4,127.3,125.2,125.1,52.1,35.1,29.5,22.9,21.5\left(\mathrm{C}=\mathrm{N}_{2}\right.$ and $\mathrm{C}=\mathrm{O}$ carbons were not observed); HRMS (ESI) $m / z$ calcd for $\mathrm{C}_{20} \mathrm{H}_{22} \mathrm{O}_{2} \mathrm{~N}_{2} \mathrm{Na}[\mathrm{M}+\mathrm{Na}]^{+}: 345.1573$ found 345.1570; $\mathrm{R}_{\mathrm{f}}$ value $=0.41$ (hexane/EtOAc 9:1).

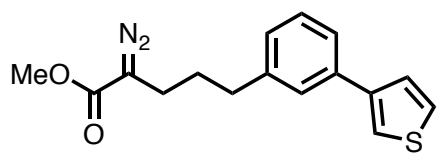

\section{Methyl 2-diazo-5-(3-(thiophen-3-yl)phenyl)pentanoate (7K)}

Following general procedure using $163.9 \mathrm{mg}$ of $\mathbf{S 3 K}, \mathbf{7 K}$ was obtained as a yellow oil $(86.4 \mathrm{mg}$, $0.288 \mathrm{mmol}, 48 \%$ yield in 2 steps). ${ }^{1} \mathrm{H}$ NMR $\left(400 \mathrm{MHz}, \mathrm{CDCl}_{3}\right) \delta 7.49-7.35(\mathrm{~m}, 5 \mathrm{H}), 7.32(\mathrm{t}, J=7.6$ $\mathrm{Hz}, 1 \mathrm{H}), 7.12(\mathrm{~d}, J=7.6 \mathrm{~Hz}, 1 \mathrm{H}), 3.76(\mathrm{~s}, 3 \mathrm{H}), 2.72(\mathrm{t}, J=7.6 \mathrm{~Hz}, 2 \mathrm{H}), 2.37$ (t, $J=7.6 \mathrm{~Hz}, 2 \mathrm{H}), 1.89$ (quintet, $J=7.6 \mathrm{~Hz}, 2 \mathrm{H}) ;{ }^{13} \mathrm{C}$ NMR $\left(101 \mathrm{MHz}, \mathrm{CDCl}_{3}\right) \delta 142.5,142.0,136.1,129.0,127.3,126.7$, 126.5, 126.3, 124.4, 120.4, 52.0, 35.0, 29.4, $22.9\left(\mathrm{C}=\mathrm{N}_{2}\right.$ and $\mathrm{C}=\mathrm{O}$ carbons were not observed $)$; HRMS (ESI) $\mathrm{m} / \mathrm{z}$ calcd for $\mathrm{C}_{16} \mathrm{H}_{16} \mathrm{O}_{2} \mathrm{~N}_{2} \mathrm{NaS}[\mathrm{M}+\mathrm{Na}]^{+}$: 323.0825 found $323.0822 ; \mathrm{R}_{\mathrm{f}}$ value $=0.40$ (hexane/EtOAc 9:1). 


\section{Rh-catalyzed asymmetric intramolecular Buchner ring expansion of $\alpha$-diazoester}

\section{3-1. General procedure}

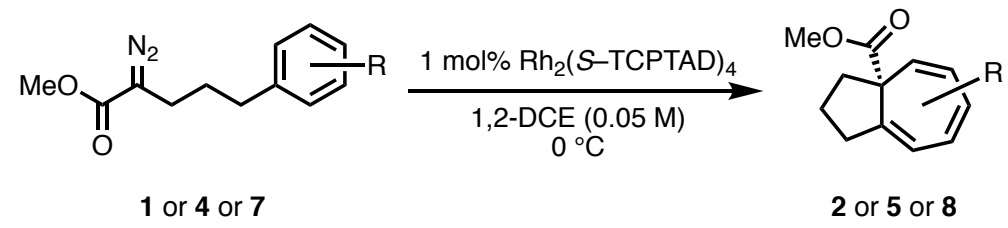

A $25-\mathrm{mL}$ glass vessel equipped with a screw cap containing a magnetic stirring bar was dried with a heat-gun in vacuo and filled with $\mathrm{N}_{2}$ after cooling to room temperature. To this vessel were added $\mathrm{Rh}_{2}(S \text {-TCPTAD })_{4}(4.2 \mathrm{mg}, 2.0 \mu \mathrm{mol}, 1 \mathrm{~mol} \%)$ and anhydrous 1,2-dichloroethane (1,2-DCE, $\left.2.0 \mathrm{~mL}\right)$. To this solution was slowly added $\alpha$-diazoester $\left(0.20 \mathrm{mmol}\right.$ in $2.0 \mathrm{~mL}$ of 1,2 -DCE) at $0{ }^{\circ} \mathrm{C}$. After stirring the mixture for 15 minutes at $0{ }^{\circ} \mathrm{C}, 1,2$-DCE was removed in vacuo. The residue was purified by Isolera ${ }^{\circledR}$ (hexane/EtOAc 97:3 to 94:6) to afford corresponding triene. The racemic samples were prepared using $\mathrm{Rh}_{2}(\mathrm{esp})_{2}$ as a catalyst instead of $\mathrm{Rh}_{2}(S \text {-TCPTAD })_{4}$.

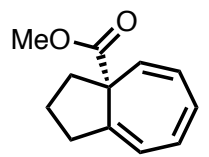

\section{Methyl $(R)$-2,3-dihydroazulene-3a(1H)-carboxylate $(2 \mathrm{~A})^{[4]}$}

Following general procedure, 2A was obtained as a colorless oil $(22.1 \mathrm{mg}, 0.116 \mathrm{mmol}, 58 \%$ yield, $91 \% e e)$. The spectra are in accordance with those of the compound reported in the literature. $[\alpha]_{\mathrm{D}}{ }^{25}$ +30.3 ( $c$ 1.00, $\mathrm{CHCl}_{3}$ ); enantiomeric excess $91 \%$ was determined by chiral HPLC analysis: Chiralpak OD-H, $n$-hexane/IPA 99:1, $1.0 \mathrm{~mL} / \mathrm{min}, 40{ }^{\circ} \mathrm{C}$, detection at $254 \mathrm{~nm}$, retention time (min): 7.55 (major) and 8.19 (minor); ${ }^{1} \mathrm{H}$ NMR (400 MHz, $\left.\mathrm{CDCl}_{3}\right) \delta 6.54(\mathrm{dd}, J=11.2,6.0 \mathrm{~Hz}, 1 \mathrm{H}), 6.45(\mathrm{dd}, J=11.2$, $6.4 \mathrm{~Hz}, 1 \mathrm{H}), 6.37-6.28(\mathrm{~m}, 2 \mathrm{H}), 5.26(\mathrm{~d}, J=8.8 \mathrm{~Hz}, 1 \mathrm{H}), 3.49$ (s, 3H), 2.82-2.71 (m, 1H), 2.64-2.56 $(\mathrm{m}, 1 \mathrm{H}), 2.32-2.18(\mathrm{~m}, 2 \mathrm{H}), 2.05-1.95(\mathrm{~m}, 1 \mathrm{H}), 1.70-1.58(\mathrm{~m}, 1 \mathrm{H}) ;{ }^{13} \mathrm{C} \mathrm{NMR}\left(101 \mathrm{MHz}, \mathrm{CDCl}_{3}\right) \delta$ 174.9, 144.3, 129.9, 127.6, 126.8, 125.6, 118.6, 55.6, 52.0, 41.8, 34.8, 26.6; HRMS (ESI) $\mathrm{m} / z$ calcd for $\mathrm{C}_{12} \mathrm{H}_{14} \mathrm{O}_{2} \mathrm{Na}[\mathrm{M}+\mathrm{Na}]^{+}: 213.0886$ found 213.0884; $\mathrm{R}_{\mathrm{f}}$ value $=0.32$ (hexane/EtOAc 9:1).

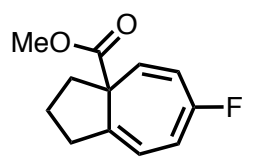

\section{Methyl 6-fluoro-2,3-dihydroazulene-3a(1H)-carboxylate (2B)}

Following general procedure, 2B was obtained (38\% NMR yield). ${ }^{1} \mathrm{H}$ NMR (400 $\left.\mathrm{MHz}, \mathrm{CDCl}_{3}\right) \delta$ 6.33-6.22 (m, 3H), 5.37 (dd, $J=10.0,5.6 \mathrm{~Hz}, 1 \mathrm{H}), 3.53$ (s, 3H), 2.79-2.69 (m, 1H), 2.59-2.51 (m, 1H), 2.29-2.24 (m, 2H), 2.05-1.94 (m, 1H), 1.69-1.58 (m, 1H); ${ }^{13} \mathrm{C}$ NMR (101 MHz, $\left.\mathrm{CDCl}_{3}\right) \delta 174.6,160.6$ $\left(\mathrm{d}, J_{\mathrm{C}-\mathrm{F}}=245.8 \mathrm{~Hz}\right), 141.1,127.8\left(\mathrm{~d}, J_{\mathrm{C}-\mathrm{F}}=14.5 \mathrm{~Hz}\right), 121.1\left(\mathrm{~d}, J_{\mathrm{C}-\mathrm{F}}=34.8 \mathrm{~Hz}\right), 115.6\left(\mathrm{~d}, J_{\mathrm{C}-\mathrm{F}}=11.6\right.$ $\mathrm{Hz}), 110.9\left(\mathrm{~d}, J_{\mathrm{C}-\mathrm{F}}=26.2 \mathrm{~Hz}\right), 55.7,52.3,41.9,34.5,26.6$; HRMS (ESI) $m / z$ calcd for $\mathrm{C}_{12} \mathrm{H}_{13} \mathrm{FO}_{2} \mathrm{Na}$ $[\mathrm{M}+\mathrm{Na}]^{+}: 231.0792$ found 231.0788; $\mathrm{R}_{\mathrm{f}}$ value $=0.45$ (hexane/EtOAc 5:1). 


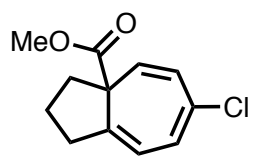

\section{Methyl 6-chloro-2,3-dihydroazulene-3a(1H)-carboxylate (2C)}

Following general procedure, $2 \mathrm{C}$ was obtained (22\% NMR yield). ${ }^{1} \mathrm{H}$ NMR $\left(400 \mathrm{MHz}, \mathrm{CDCl}_{3}\right) \delta$ $6.69(\mathrm{~d}, J=7.2 \mathrm{~Hz}, 1 \mathrm{H}), 6.28(\mathrm{~d}, J=10.0 \mathrm{~Hz}, 1 \mathrm{H}), 6.24(\mathrm{~d}, J=7.2 \mathrm{~Hz}, 1 \mathrm{H}), 5.28(\mathrm{~d}, J=10.0 \mathrm{~Hz}, 1 \mathrm{H})$, 3.54 (s, 3H), 2.79-2.69 (m, 1H), 2.60-2.52 (m, 1H), 2.28-2.23 (m, 2H), 2.05-1.95 (m, 1H), 1.69-1.59 $(\mathrm{m}, 1 \mathrm{H}) ;{ }^{13} \mathrm{C}$ NMR $\left(101 \mathrm{MHz}, \mathrm{CDCl}_{3}\right) \delta 174.3,144.9,132.8,128.6,128.3,127.1,117.4,55.7,52.3$, 41.5, 34.5, 26.5; HRMS (ESI) $\mathrm{m} / z$ calcd for $\mathrm{C}_{12} \mathrm{H}_{13} \mathrm{ClO}_{2} \mathrm{Na}[\mathrm{M}+\mathrm{Na}]^{+}: 247.0496$ found 247.0492; $\mathrm{R}_{\mathrm{f}}$ value $=0.46($ hexane/EtOAc 5:1)

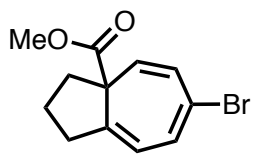

\section{Methyl 6-bromo-2,3-dihydroazulene-3a(1H)-carboxylate (2D)}

Following general procedure, 2D was obtained (17\% NMR yield). ${ }^{1} \mathrm{H}$ NMR (400 $\left.\mathrm{MHz}, \mathrm{CDCl}_{3}\right) \delta$ $6.91(\mathrm{~d}, J=6.8 \mathrm{~Hz}, 1 \mathrm{H}), 6.39(\mathrm{~d}, J=10.0 \mathrm{~Hz}, 1 \mathrm{H}), 6.18(\mathrm{~d}, J=6.8 \mathrm{~Hz}, 1 \mathrm{H}), 5.20(\mathrm{~d}, J=10.0 \mathrm{~Hz}, 1 \mathrm{H})$, $3.54(\mathrm{~s}, 3 \mathrm{H}), 2.75-2.65(\mathrm{~m}, 1 \mathrm{H}), 2.59-2.50(\mathrm{~m}, 1 \mathrm{H}), 2.27-2.22(\mathrm{~m}, 2 \mathrm{H}), 2.05-1.96(\mathrm{~m}, 1 \mathrm{H}), 1.69-1.59$ $(\mathrm{m}, 1 \mathrm{H}) ;{ }^{13} \mathrm{C} \mathrm{NMR}\left(101 \mathrm{MHz}, \mathrm{CDCl}_{3}\right) \delta 174.3,145.6,132.4,130.2,127.2,122.0,118.2,55.8,52.3$, 41.4, 34.5, 26.5; HRMS (ESI) $\mathrm{m} / z$ calcd for $\mathrm{C}_{12} \mathrm{H}_{13} \mathrm{BrO}_{2} \mathrm{Na}[\mathrm{M}+\mathrm{Na}]^{+}: 290.9991$ found 290.9987; $\mathrm{R}_{\mathrm{f}}$ value $=0.49($ hexane/EtOAc 5:1)

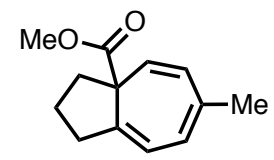

\section{Methyl 6-methyl-2,3-dihydroazulene-3a(1H)-carboxylate (2E)}

Following general procedure, $2 \mathbf{E}$ was obtained (60\% NMR yield). ${ }^{1} \mathrm{H}$ NMR (400 $\left.\mathrm{MHz}, \mathrm{CDCl}_{3}\right) \delta$ $6.31(\mathrm{~d}, J=6.4 \mathrm{~Hz}, 1 \mathrm{H}), 6.21(\mathrm{~d}, J=6.4 \mathrm{~Hz}, 1 \mathrm{H}), 6.13(\mathrm{~d}, J=9.6 \mathrm{~Hz}, 1 \mathrm{H}), 5.24(\mathrm{~d}, J=9.6 \mathrm{~Hz}, 1 \mathrm{H})$, 3.49 (s, 3H), 2.78-2.67 (m, 1H), 2.60-2.51 (m, 1H), 2.27-2.15 (m, 2H), 2.01 (s, 3H), 2.00-1.93 (m, 1H), 1.70-1.59 (m, 1H); ${ }^{13} \mathrm{C}$ NMR (101 MHz, $\left.\mathrm{CDCl}_{3}\right) \delta 175.4,142.4,136.7,129.9,127.1,125.0,118.3$, 55.3, 52.0, 41.6, 34.4, 26.7, 24.1; HRMS (ESI) $m / z$ calcd for $\mathrm{C}_{13} \mathrm{H}_{16} \mathrm{O}_{2} \mathrm{Na}[\mathrm{M}+\mathrm{Na}]^{+}: 227.1043$ found 227.1039; $\mathrm{R}_{\mathrm{f}}$ value $=0.38$ (hexane/EtOAc 9:1)

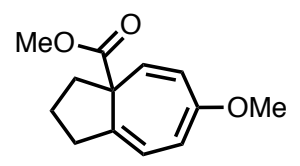

Methyl 6-methoxy-2,3-dihydroazulene-3a(1H)-carboxylate (2F) 
Following general procedure, $2 \mathrm{~F}$ was obtained (41\% NMR yield). ${ }^{1} \mathrm{H}$ NMR (400 $\left.\mathrm{MHz}, \mathrm{CDCl}_{3}\right) \delta$ $6.21(\mathrm{~d}, J=6.8 \mathrm{~Hz}, 1 \mathrm{H}), 6.17(\mathrm{~d}, J=10.0 \mathrm{~Hz}, 1 \mathrm{H}), 5.78(\mathrm{~d}, J=6.8 \mathrm{~Hz}, 1 \mathrm{H}), 5.38(\mathrm{~d}, J=10.0 \mathrm{~Hz}, 1 \mathrm{H})$, $3.60(\mathrm{~s}, 3 \mathrm{H}), 3.52(\mathrm{~s}, 3 \mathrm{H}), 2.76-2.66(\mathrm{~m}, 1 \mathrm{H}), 2.58-2.49(\mathrm{~m}, 1 \mathrm{H}), 2.28-2.18(\mathrm{~m}, 2 \mathrm{H}), 2.01-1.92(\mathrm{~m}, 1 \mathrm{H})$, 1.71-1.60 (m, 1H); ${ }^{13} \mathrm{C}$ NMR (101 MHz, $\left.\mathrm{CDCl}_{3}\right) \delta 175.3,158.0,139.1,127.9,124.2,116.8,104.2$, 55.3, 55.1, 52.1, 41.8, 34.3, 26.6; HRMS (ESI) $\mathrm{m} / z$ calcd for $\mathrm{C}_{13} \mathrm{H}_{17} \mathrm{O}_{3}[\mathrm{M}+\mathrm{H}]^{+}: 221.1172$ found $221.1169 ; \mathrm{R}_{\mathrm{f}}$ value $=0.38$ (hexane/EtOAc 5:1).

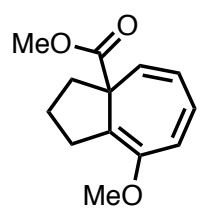

\section{Methyl 8-methoxy-2,3-dihydroazulene-3a(1H)-carboxylate (5)}

Following general procedure, 5 was obtained (24\% NMR yield). ${ }^{1} \mathrm{H}$ NMR (400 $\left.\mathrm{MHz}, \mathrm{CDCl}_{3}\right) \delta$ 6.50-6.42 (m, 2H), 6.16 (dd, $J=9.2,5.2 \mathrm{~Hz}, 1 \mathrm{H}), 5.26$ (d, $J=9.2 \mathrm{~Hz}, 1 \mathrm{H}), 3.63$ (s, 3H), 3.49 (s, 3H), 2.71-2.56 (m, 2H), 2.27-2.13 (m, 2H), 1.98-1.90 (m, 1H), 1.61-1.51 (m, 1H); ${ }^{13} \mathrm{C}$ NMR $(101 \mathrm{MHz}$, $\left.\mathrm{CDCl}_{3}\right) \delta 175.1,148.6,129.2,124.9,124.4,124.0,59.0,51.9,51.3,41.3,31.0,26.0$ (one peak is missing due to overlapping); HRMS (ESI) $\mathrm{m} / z$ calcd for $\mathrm{C}_{13} \mathrm{H}_{16} \mathrm{O}_{3} \mathrm{Na}[\mathrm{M}+\mathrm{Na}]^{+}: 243.0992$ found 243.0988; $\mathrm{R}_{\mathrm{f}}$ value $=0.36$ (hexane/EtOAc 5:1).

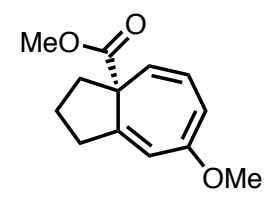

\section{Methyl $(R)$-7-methoxy-2,3-dihydroazulene-3a(1H)-carboxylate $(8 \mathrm{~A})$}

Following general procedure, 8A was obtained as a white solid $(32.9 \mathrm{mg}, 0.149 \mathrm{mmol}, 75 \%$ yield, $83 \% e e) .[\alpha]_{\mathrm{D}}{ }^{30}+189.4\left(c 1.00, \mathrm{CHCl}_{3}\right)$; enantiomeric excess $83 \%$ was determined by chiral HPLC analysis: Chiralpak OD-H, $n$-hexane/IPA $99: 1,1.0 \mathrm{~mL} / \mathrm{min}, 40{ }^{\circ} \mathrm{C}$, detection at $254 \mathrm{~nm}$, retention time (min): 8.47 (minor) and 10.42 (major); $\left.{ }^{1} \mathrm{H} \mathrm{NMR} \mathrm{(400} \mathrm{MHz,} \mathrm{CDCl}_{3}\right) \delta 6.23$ (dd, $J=9.2,7.2 \mathrm{~Hz}, 1 \mathrm{H}$ ), 6.19 (s, 1H), 5.68 (d, $J=7.2 \mathrm{~Hz}, 1 \mathrm{H}), 5.09$ (d, $J=9.2 \mathrm{~Hz}, 1 \mathrm{H}), 3.61$ (s, 3H), 3.52 (s, 3H), 2.79-2.68 (m, $1 \mathrm{H}), 2.65-2.56(\mathrm{~m}, 1 \mathrm{H}), 2.28-2.15(\mathrm{~m}, 2 \mathrm{H}), 2.01-1.92(\mathrm{~m}, 1 \mathrm{H}), 1.70-1.58(\mathrm{~m}, 1 \mathrm{H}) ;{ }^{13} \mathrm{C}$ NMR $(101$ $\left.\mathrm{MHz}, \mathrm{CDCl}_{3}\right) \delta 175.3,159.8,146.0,125.9,121.5,116.0,101.8,55.5,55.0,52.1,41.7,35.1$, 26.4; HRMS (ESI) $m / z$ calcd for $\mathrm{C}_{13} \mathrm{H}_{17} \mathrm{O}_{3}[\mathrm{M}+\mathrm{H}]^{+}: 221.1172$ found $221.1169 ; \mathrm{R}_{\mathrm{f}}$ value $=0.44$ (hexane/EtOAc 4:1).

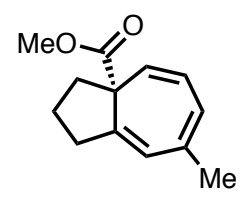

Methyl (R)-7-methyl-2,3-dihydroazulene-3a(1H)-carboxylate (8B) 
Following general procedure, $\mathbf{8 B}$ was obtained as a colorless oil $(27.1 \mathrm{mg}, 0.133 \mathrm{mmol}, 66 \%$ yield, $87 \% e e) \cdot[\alpha]_{\mathrm{D}}^{28}+41.0\left(c 1.00, \mathrm{CHCl}_{3}\right)$; enantiomeric excess $87 \%$ was determined by chiral HPLC analysis: Chiralpak AD-H, $n$-hexane/IPA 99:1, $0.3 \mathrm{~mL} / \mathrm{min}, 27^{\circ} \mathrm{C}$, detection at $254 \mathrm{~nm}$, retention time (min): 19.30 (minor) and 20.15 (major); ${ }^{1} \mathrm{H}$ NMR (400 MHz, $\left.\mathrm{CDCl}_{3}\right) \delta 6.25-6.17$ (m, 3H), 5.19 (d, $J=$ $9.2 \mathrm{~Hz}, 1 \mathrm{H}), 3.50(\mathrm{~s}, 3 \mathrm{H}), 2.77-2.67(\mathrm{~m}, 1 \mathrm{H}), 2.63-2.54(\mathrm{~m}, 1 \mathrm{H}), 2.28-2.15(\mathrm{~m}, 2 \mathrm{H}), 2.02(\mathrm{~s}, 3 \mathrm{H})$, 2.01-1.92 (m, 1H), 1.69-1.58 (m, 1H); ${ }^{13} \mathrm{C}$ NMR (101 MHz, $\left.\mathrm{CDCl}_{3}\right) \delta 175.2,143.2,139.3,126.8$, 125.0, 124.1, 121.6, 55.3, 52.0, 41.6, 34.8, 26.6, 24.4; HRMS (ESI) $m / z$ calcd for $\mathrm{C}_{13} \mathrm{H}_{17} \mathrm{O}_{2}[\mathrm{M}+\mathrm{H}]^{+}$: 205.1223 found 205.1221; $R_{\mathrm{f}}$ value $=0.40$ (hexane/EtOAc 9:1).

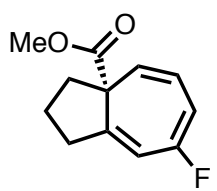

\section{Methyl (R)-7-fluoro-2,3-dihydroazulene-3a(1H)-carboxylate (8C)}

Following general procedure, $\mathbf{8 C}$ was obtained as a white solid $(23.5 \mathrm{mg}, 0.112 \mathrm{mmol}, 56 \%$ yield, $96 \% e e) \cdot[\alpha]_{\mathrm{D}}^{29}+158.3\left(c 1.00, \mathrm{CHCl}_{3}\right.$ ); enantiomeric excess $96 \%$ was determined by chiral HPLC analysis: Chiralpak AD-H, $n$-hexane/IPA $99: 1,1.0 \mathrm{~mL} / \mathrm{min}, 40^{\circ} \mathrm{C}$, detection at $254 \mathrm{~nm}$, retention time (min): 6.46 (minor) and 7.36 (major); ${ }^{1} \mathrm{H}$ NMR (400 MHz, $\left.\mathrm{CDCl}_{3}\right) \delta$ 6.34-6.28 (m, 1H), 6.28-6.22 (m, $1 \mathrm{H}), 6.19-6.10(\mathrm{~m}, 1 \mathrm{H}), 5.13(\mathrm{~d}, J=9.6 \mathrm{~Hz}, 1 \mathrm{H}), 3.53(\mathrm{~s}, 3 \mathrm{H}), 2.84-2.73(\mathrm{~m}, 1 \mathrm{H}), 2.67-2.58(\mathrm{~m}, 1 \mathrm{H})$, 2.30-2.22 (m, 2H), 2.05-1.97 (m, 1H), 1.71-1.58 (m, 1H); ${ }^{13} \mathrm{C}$ NMR $\left(101 \mathrm{MHz}, \mathrm{CDCl}_{3}\right) \delta 174.5,162.1$ (d, $J=249.6 \mathrm{~Hz}), 147.0$ (d, $J=14.4 \mathrm{~Hz}), 124.5$ (d, $J=11.6 \mathrm{~Hz}), 123.1(\mathrm{~d}, J=2.9 \mathrm{~Hz}), 113.1$ (d, $J=$ $35.9 \mathrm{~Hz}$ ), 108.5 (d, $J=27.1 \mathrm{~Hz}$ ), 56.1, 52.3, 41.8, 35.1, 26.5; HRMS (ESI) $\mathrm{m} / \mathrm{z}$ calcd for $\mathrm{C}_{12} \mathrm{H}_{14} \mathrm{O}_{2} \mathrm{~F}$ $[\mathrm{M}+\mathrm{H}]^{+}: 209.0972$ found 209.0973; $\mathrm{R}_{\mathrm{f}}$ value $=0.34$ (hexane/EtOAc 9:1).

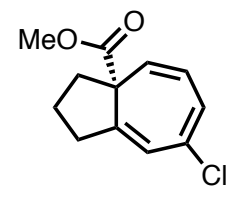

\section{Methyl $(R)$-7-chloro-2,3-dihydroazulene-3a(1H)-carboxylate (8D)}

Following general procedure, 8D was obtained as a white solid $(28.7 \mathrm{mg}, 0.128 \mathrm{mmol}, 64 \%$ yield, $94 \% e e) \cdot[\alpha]_{\mathrm{D}}{ }^{25}+37.6\left(c 1.00, \mathrm{CHCl}_{3}\right)$; enantiomeric excess $94 \%$ was determined by chiral HPLC analysis: Chiralpak AD-H, $n$-hexane/IPA 99:1, $1.0 \mathrm{~mL} / \mathrm{min}, 40^{\circ} \mathrm{C}$, detection at $254 \mathrm{~nm}$, retention time (min): 6.21 (minor) and 6.91 (major); ${ }^{1} \mathrm{H}$ NMR $\left(400 \mathrm{MHz}, \mathrm{CDCl}_{3}\right) \delta 6.60(\mathrm{~d}, J=6.8 \mathrm{~Hz}, 1 \mathrm{H}), 6.33$ (s, $1 \mathrm{H}), 6.22(\mathrm{dd}, J=9.6,6.8 \mathrm{~Hz}, 1 \mathrm{H}), 5.27$ (d, $J=9.6 \mathrm{~Hz}, 1 \mathrm{H}), 3.54(\mathrm{~s}, 3 \mathrm{H}), 2.82-2.71(\mathrm{~m}, 1 \mathrm{H})$, 2.65-2.56 (m, 1H), 2.31-2.21 (m, 2H), 2.05-1.95 (m, 1H), 1.69-1.57 (m, 1H); ${ }^{13} \mathrm{C}$ NMR $(101 \mathrm{MHz}$, $\left.\mathrm{CDCl}_{3}\right) \delta 174.2,146.1,135.5,126.3,126.2,125.8,120.3,55.9,52.3,41.5,34.8,26.5$; HRMS (ESI) $m / z$ calcd for $\mathrm{C}_{12} \mathrm{H}_{14} \mathrm{O}_{2} \mathrm{Cl}[\mathrm{M}+\mathrm{H}]^{+}: 225.0677$ found 225.0674; $\mathrm{R}_{\mathrm{f}}$ value $=0.34$ (hexane/EtOAc 9:1). 


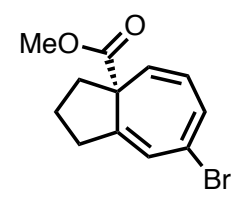

\section{Methyl (R)-7-bromo-2,3-dihydroazulene-3a(1H)-carboxylate (8E)}

Following general procedure, $\mathbf{8 E}$ was obtained as a white solid $(32.8 \mathrm{mg}, 0.122 \mathrm{mmol}, 61 \%$ yield, $96 \% e e) .[\alpha]_{\mathrm{D}}^{30}-6.5\left(c 1.00, \mathrm{CHCl}_{3}\right.$ ); enantiomeric excess $96 \%$ was determined by chiral HPLC analysis: Chiralpak AD-H, $n$-hexane/IPA $99: 1,1.0 \mathrm{~mL} / \mathrm{min}, 40{ }^{\circ} \mathrm{C}$, detection at $254 \mathrm{~nm}$, retention time (min): 7.01 (minor) and 7.71 (major); ${ }^{1} \mathrm{H}$ NMR $\left(400 \mathrm{MHz}, \mathrm{CDCl}_{3}\right) \delta 6.83(\mathrm{~d}, J=7.2 \mathrm{~Hz}, 1 \mathrm{H}), 6.44$ (s, $1 \mathrm{H}), 6.16(\mathrm{dd}, J=9.6,7.2 \mathrm{~Hz}, 1 \mathrm{H}), 5.31(\mathrm{~d}, J=9.6 \mathrm{~Hz}, 1 \mathrm{H}), 3.54(\mathrm{~s}, 3 \mathrm{H}), 2.81-2.70(\mathrm{~m}, 1 \mathrm{H})$, 2.64-2.55 (m, 1H), 2.30-2.18 (m, 2H), 2.04-1.95 (m, 1H), 1.68-1.56 (m, 1H); ${ }^{13} \mathrm{C}$ NMR $(101 \mathrm{MHz}$, $\left.\mathrm{CDCl}_{3}\right) \delta 174.1,146.3,130.0,126.8,126.5,125.1,122.3,55.9,52.3,41.4,34.6,26.5$; HRMS (ESI) $m / z$ calcd for $\mathrm{C}_{12} \mathrm{H}_{14} \mathrm{O}_{2} \mathrm{Br}[\mathrm{M}+\mathrm{H}]^{+}: 269.0172$ found 269.0172; $\mathrm{R}_{\mathrm{f}}$ value $=0.35$ (hexane/EtOAc 9:1); m.p. $103^{\circ} \mathrm{C}$.

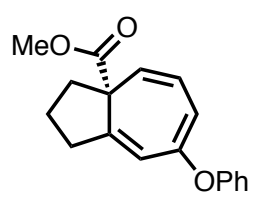

\section{Methyl $(R)$-7-phenoxy-2,3-dihydroazulene-3a(1H)-carboxylate $(8 \mathrm{~F})$}

Following general procedure, $\mathbf{8 F}$ was obtained as a colorless oil $(47.0 \mathrm{mg}, 0.166 \mathrm{mmol}, 83 \%$ yield, $81 \% e e) \cdot[\alpha]_{\mathrm{D}}^{29}+19.7\left(c 1.00, \mathrm{CHCl}_{3}\right)$; enantiomeric excess $81 \%$ was determined by chiral HPLC analysis: Chiralpak OZ-H, $n$-hexane/IPA $99: 1,1.0 \mathrm{~mL} / \mathrm{min}, 40{ }^{\circ} \mathrm{C}$, detection at $254 \mathrm{~nm}$, retention time (min): 6.19 (minor) and 9.07 (major); ${ }^{1} \mathrm{H}$ NMR $\left(400 \mathrm{MHz}, \mathrm{CDCl}_{3}\right) \delta 7.29$ (t, $\left.J=8.0 \mathrm{~Hz}, 2 \mathrm{H}\right), 7.04$ (t, $J$ $=8.0 \mathrm{~Hz}, 1 \mathrm{H}), 6.98(\mathrm{~d}, J=8.0 \mathrm{~Hz}, 2 \mathrm{H}), 6.29(\mathrm{dd}, J=9.2,7.2 \mathrm{~Hz}, 1 \mathrm{H}), 6.24(\mathrm{~s}, 1 \mathrm{H}), 6.13(\mathrm{~d}, J=7.2 \mathrm{~Hz}$, $1 \mathrm{H}), 5.16(\mathrm{~d}, J=9.2 \mathrm{~Hz}, 1 \mathrm{H}), 3.61(\mathrm{~s}, 3 \mathrm{H}), 2.77-2.66(\mathrm{~m}, 1 \mathrm{H}), 2.62-2.54(\mathrm{~m}, 1 \mathrm{H}), 2.33-2.20(\mathrm{~m}, 2 \mathrm{H})$, 2.03-1.92 (m, 1H), 1.68-1.57 (m, 1H); ${ }^{13} \mathrm{C}$ NMR (101 MHz, $\left.\mathrm{CDCl}_{3}\right) \delta 174.9,157.1,155.9,145.2$, 129.6, 125.8, 122.9, 122.6, 118.4, 116.7, 113.0, 55.9, 52.1, 42.0, 35.2, 26.4; HRMS (ESI) $\mathrm{m} / \mathrm{z}$ calcd for $\mathrm{C}_{18} \mathrm{H}_{19} \mathrm{O}_{3}[\mathrm{M}+\mathrm{H}]^{+}: 283.1329$ found 283.1324; $\mathrm{R}_{\mathrm{f}}$ value $=0.24$ (hexane/EtOAc 9:1).

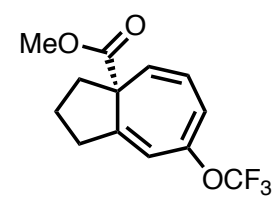

\section{Methyl (R)-7-(trifluoromethoxy)-2,3-dihydroazulene-3a(1H)-carboxylate (8G)}

Following general procedure, 8G was obtained as a colorless oil $(26.0 \mathrm{mg}, 0.0948 \mathrm{mmol}, 48 \%$ yield, $91 \% e e) \cdot[\alpha]_{\mathrm{D}}{ }^{25}+93.9\left(c 1.00, \mathrm{CHCl}_{3}\right)$; enantiomeric excess $91 \%$ was determined by chiral HPLC analysis: Chiralpak AD-H, $n$-hexane/IPA 99:1, $1.0 \mathrm{~mL} / \mathrm{min}, 40^{\circ} \mathrm{C}$, detection at $254 \mathrm{~nm}$, retention time (min): 5.03 (minor) and 5.31 (major); ${ }^{1} \mathrm{H}$ NMR (400 MHz, $\left.\mathrm{CDCl}_{3}\right) \delta 6.43(\mathrm{~d}, J=7.2 \mathrm{~Hz}, 1 \mathrm{H})$, 6.37-6.28 (m, 2H), $5.29(\mathrm{~d}, J=9.2 \mathrm{~Hz}, 1 \mathrm{H}), 3.49(\mathrm{~s}, 3 \mathrm{H}), 2.86-2.75(\mathrm{~m}, 1 \mathrm{H}), 2.67-2.59(\mathrm{~m}, 1 \mathrm{H})$, 
2.34-2.22 (m, 2H), 2.08-1.99 (m, 1H), 1.68-1.56 (m, 1H); $\left.{ }^{13} \mathrm{C} \mathrm{NMR} \mathrm{(101} \mathrm{MHz,} \mathrm{CDCl}_{3}\right) \delta$ 174.0, 149.1, 146.5, 126.4, 124.8, 120.8 (q, $J=260.3 \mathrm{~Hz}$ ), 119.0, 115.2, 56.0, 52.1, 41.5, 34.9, 26.6; HRMS (ESI) $\mathrm{m} / z$ calcd for $\mathrm{C}_{13} \mathrm{H}_{13} \mathrm{O}_{3} \mathrm{~F}_{3} \mathrm{Na}[\mathrm{M}+\mathrm{Na}]^{+}: 297.0709$ found 297.0707; $\mathrm{R}_{\mathrm{f}}$ value $=0.29$ (hexane/EtOAc 9:1).

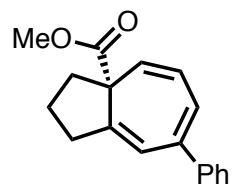

\section{Methyl (R)-7-phenyl-2,3-dihydroazulene-3a(1H)-carboxylate $(8 \mathrm{H})$}

Following general procedure, $\mathbf{8 H}$ was obtained as a colorless oil $(41.2 \mathrm{mg}, 0.155 \mathrm{mmol}, 77 \%$ yield, $77 \% e e)$. $[\alpha]_{\mathrm{D}}{ }^{25}-12.4$ (c 1.00, $\mathrm{CHCl}_{3}$ ); enantiomeric excess $77 \%$ was determined by chiral HPLC analysis: Chiralpak AD-H, $n$-hexane/IPA 99:1, $1.0 \mathrm{~mL} / \mathrm{min}, 40{ }^{\circ} \mathrm{C}$, detection at $254 \mathrm{~nm}$, retention time (min): 7.88 (minor) and 8.86 (major); ${ }^{1} \mathrm{H}$ NMR $\left(400 \mathrm{MHz}, \mathrm{CDCl}_{3}\right) \delta 7.43$ (d, J=8.0 Hz, 2H), 7.35 (t, $J=8.0 \mathrm{~Hz}, 2 \mathrm{H}), 7.30-7.26(\mathrm{~m}, 1 \mathrm{H}), 6.74(\mathrm{~d}, J=6.4 \mathrm{~Hz}, 1 \mathrm{H}), 6.47(\mathrm{~s}, 1 \mathrm{H}), 6.41(\mathrm{dd}, J=9.6,6.4 \mathrm{~Hz}$, $1 \mathrm{H}), 5.38(\mathrm{~d}, J=9.6 \mathrm{~Hz}, 1 \mathrm{H}), 3.44(\mathrm{~s}, 3 \mathrm{H}), 2.88-2.77(\mathrm{~m}, 1 \mathrm{H}), 2.72-2.64(\mathrm{~m}, 1 \mathrm{H}), 2.36-2.19(\mathrm{~m}, 2 \mathrm{H})$, 2.07-1.98 (m, 1H), 1.75-1.62 (m, 1H); ${ }^{13} \mathrm{C}$ NMR (101 MHz, $\left.\mathrm{CDCl}_{3}\right) \delta 174.9,144.9,142.9,142.6$, 128.5, 127.4, 127.3, 127.1, 126.6, 125.3, 119.7, 55.5, 52.0, 41.5, 34.9, 26.6; HRMS (ESI) $\mathrm{m} / z$ calcd for $\mathrm{C}_{18} \mathrm{H}_{19} \mathrm{O}_{2}[\mathrm{M}+\mathrm{H}]^{+}: 267.1380$ found 267.1378; $\mathrm{R}_{\mathrm{f}}$ value $=0.36$ (hexane/EtOAc 9:1).

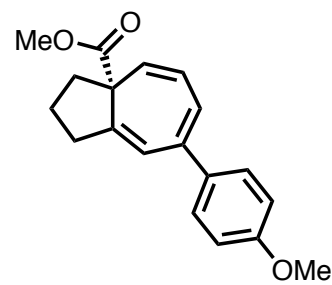

\section{Methyl (R)-7-(4-methoxyphenyl)-2,3-dihydroazulene-3a(1H)-carboxylate (8I)}

Following general procedure, 8I was obtained as a colorless oil (44.4 mg, $0.150 \mathrm{mmol}, 75 \%$ yield, $77 \% e e) .[\alpha]_{D}^{27}-26.4\left(c 1.00, \mathrm{CHCl}_{3}\right)$; enantiomeric excess $77 \%$ was determined by chiral HPLC analysis: Chiralpak AD-H, $n$-hexane/IPA 99:1, $1.0 \mathrm{~mL} / \mathrm{min}, 40{ }^{\circ} \mathrm{C}$, detection at $254 \mathrm{~nm}$, retention time (min): 20.43 (major) and 23.33 (minor); $\left.{ }^{1} \mathrm{H} \mathrm{NMR} \mathrm{(400} \mathrm{MHz,} \mathrm{CDCl}_{3}\right) \delta 7.37$ (d, $\left.J=8.8 \mathrm{~Hz}, 2 \mathrm{H}\right), 6.89$ $(\mathrm{d}, J=8.8 \mathrm{~Hz}, 2 \mathrm{H}), 6.68(\mathrm{~d}, J=6.4 \mathrm{~Hz}, 1 \mathrm{H}), 6.43(\mathrm{~s}, 1 \mathrm{H}), 6.38(\mathrm{dd}, J=9.6,6.4 \mathrm{~Hz}, 1 \mathrm{H}), 5.34(\mathrm{~d}, J=$ $9.6 \mathrm{~Hz}, 1 \mathrm{H}), 3.81(\mathrm{~s}, 3 \mathrm{H}), 3.43(\mathrm{~s}, 3 \mathrm{H}), 2.87-2.76(\mathrm{~m}, 1 \mathrm{H}), 2.70-2.62(\mathrm{~m}, 1 \mathrm{H}), 2.35-2.18(\mathrm{~m}, 2 \mathrm{H})$, 2.07-1.97 (m, 1H), 1.73-1.62 (m, 1H); ${ }^{13} \mathrm{C}$ NMR (101 MHz, $\left.\mathrm{CDCl}_{3}\right) \delta 175.0,159.3,144.7,142.4$, 135.2, 128.4, 127.1, 126.2, 124.2, 119.8, 113.9, 55.5, 55.4, 52.0, 41.5, 34.8, 26.6; HRMS (ESI) $\mathrm{m} / \mathrm{z}$ calcd for $\mathrm{C}_{19} \mathrm{H}_{21} \mathrm{O}_{3}[\mathrm{M}+\mathrm{H}]^{+}: 297.1485$ found 297.1483; $\mathrm{R}_{\mathrm{f}}$ value $=0.22$ (hexane/EtOAc 9:1). 


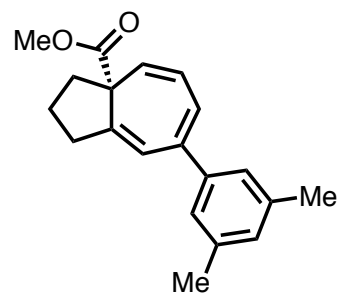

\section{Methyl (R)-7-(3,5-dimethylphenyl)-2,3-dihydroazulene-3a(1H)-carboxylate (8J)}

Following general procedure, 8J was obtained as a white solid $(33.3 \mathrm{mg}, 0.113 \mathrm{mmol}, 57 \%$ yield, $75 \% e e)$. $[\alpha]_{\mathrm{D}}{ }^{28}-16.4$ (c 1.00, $\mathrm{CHCl}_{3}$ ); enantiomeric excess $75 \%$ was determined by chiral HPLC analysis: Chiralpak IF, $n$-hexane/IPA $99: 1,1.0 \mathrm{~mL} / \mathrm{min}, 40{ }^{\circ} \mathrm{C}$, detection at $254 \mathrm{~nm}$, retention time (min): 7.35 (minor) and 7.87 (major); ${ }^{1} \mathrm{H}$ NMR $\left(400 \mathrm{MHz}, \mathrm{CDCl}_{3}\right) \delta 7.05$ (s, 2H), $6.94(\mathrm{~s}, 1 \mathrm{H}), 6.72$ (d, $J=6.4 \mathrm{~Hz}, 1 \mathrm{H}), 6.46(\mathrm{~s}, 1 \mathrm{H}), 6.39(\mathrm{dd}, J=9.6,6.4 \mathrm{~Hz}, 1 \mathrm{H}), 5.35$ (d, $J=9.6 \mathrm{~Hz}, 1 \mathrm{H}), 3.44$ (s, 3H), 2.89-2.78 (m, 1H), 2.70-2.62 (m, 1H), 2.34-2.16 (m, 2H), 2.31 (s, 6H), 2.07-1.97 (m, 1H), 1.73-1.61 $(\mathrm{m}, 1 \mathrm{H}) ;{ }^{13} \mathrm{C}$ NMR $\left(101 \mathrm{MHz}, \mathrm{CDCl}_{3}\right) \delta 175.0,144.5,143.1,142.6,138.0,129.2,127.1,126.3,125.2$, 125.0, 119.9, 55.5, 52.0, 41.5, 34.9, 26.6, 21.5; HRMS (ESI) $\mathrm{m} / z$ calcd for $\mathrm{C}_{20} \mathrm{H}_{23} \mathrm{O}_{2}[\mathrm{M}+\mathrm{H}]^{+}$: 295.1693 found 295.1689; $\mathrm{R}_{\mathrm{f}}$ value $=0.39$ (hexane/EtOAc 9:1).

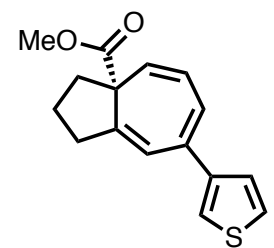

\section{Methyl (R)-7-(thiophen-3-yl)-2,3-dihydroazulene-3a(1H)-carboxylate (8K)}

Following general procedure, $\mathbf{8 K}$ was obtained as a white solid $(31.8 \mathrm{mg}, 0.117 \mathrm{mmol}, 58 \%$ yield, $81 \% e e) \cdot[\alpha]_{\mathrm{D}}{ }^{26}+2.8\left(c 1.00, \mathrm{CHCl}_{3}\right)$; enantiomeric excess $81 \%$ was determined by chiral HPLC analysis: Chiralpak AD-H, $n$-hexane/IPA $99: 1,1.0 \mathrm{~mL} / \mathrm{min}, 40{ }^{\circ} \mathrm{C}$, detection at $254 \mathrm{~nm}$, retention time (min): 13.34 (major) and 15.92 (minor); ${ }^{1} \mathrm{H}$ NMR (400 MHz, $\left.\mathrm{CDCl}_{3}\right) \delta$ 7.31-7.22 (m, 3H), 6.79 (d, $J=$ $6.8 \mathrm{~Hz}, 1 \mathrm{H}), 6.55(\mathrm{~s}, 1 \mathrm{H}), 6.37(\mathrm{dd}, J=9.6,6.8 \mathrm{~Hz}, 1 \mathrm{H}), 5.35$ (d, $J=9.6 \mathrm{~Hz}, 1 \mathrm{H}), 3.44(\mathrm{~s}, 3 \mathrm{H})$, 2.87-2.77 (m, 1H), 2.72-2.63 (m, 1H), 2.34-2.18 (m, 2H), 2.06-1.97 (m, $1 \mathrm{H}), 1.73-1.61(\mathrm{~m}, 1 \mathrm{H}) ;{ }^{13} \mathrm{C}$ NMR $\left(101 \mathrm{MHz}, \mathrm{CDCl}_{3}\right) \delta 174.9,144.8,143.8,137.0,127.1,126.5,126.2,125.7,124.2,121.2,119.0$, 55.5, 52.0, 41.6, 34.9, 26.6; HRMS (ESI) $m / z$ calcd for $\mathrm{C}_{16} \mathrm{H}_{17} \mathrm{O}_{2} \mathrm{~S}[\mathrm{M}+\mathrm{H}]^{+}: 273.0944$ found 273.0941; $\mathrm{R}_{\mathrm{f}}$ value $=0.31$ (hexane/EtOAc 9:1) 


\section{3-2. $1 \mathrm{mmol}$ scale reaction for $1 \mathrm{~A}$}

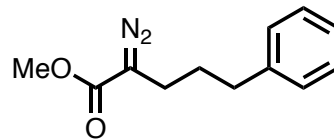

$1 \mathrm{~A}$

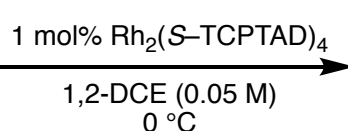

$66 \%$ yield, $91 \% e e$

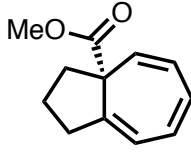

$2 \mathrm{~A}$

A $100-\mathrm{mL}$ of round-bottom flask containing a magnetic stirring bar was dried with a heat-gun in vacuo and filled with $\mathrm{N}_{2}$ after cooling to room temperature. To the flask were added $\mathrm{Rh}_{2}(S \text {-TCPTAD })_{4}$ (21.1 $\mathrm{mg}, 10.0 \mu \mathrm{mol}, 1 \mathrm{~mol} \%)$ and anhydrous 1,2-DCE $(10.0 \mathrm{~mL})$. To this solution was slowly added $\alpha$-diazoester $1 \mathrm{~A}(1 \mathrm{mmol}$ in $10.0 \mathrm{~mL}$ of $1,2-\mathrm{DCE})$ at $0{ }^{\circ} \mathrm{C}$. After stirring the mixture for 15 minutes at $0{ }^{\circ} \mathrm{C}, 1,2-\mathrm{DCE}$ was removed in vacuo. The residue was purified by Isolera ${ }^{\circledR}$ (hexane/EtOAc 97:3 to 94:6) to afford $\mathbf{2 A}$ (66\% yield, $91 \% e e$ ). 


\section{4. ${ }^{1} \mathrm{H}$ and ${ }^{13} \mathrm{C}$ NMR Spectra}

${ }^{1} \mathrm{H}$ NMR of $\mathbf{S 2 A}\left(400 \mathrm{MHz}, \mathrm{CDCl}_{3}\right)$

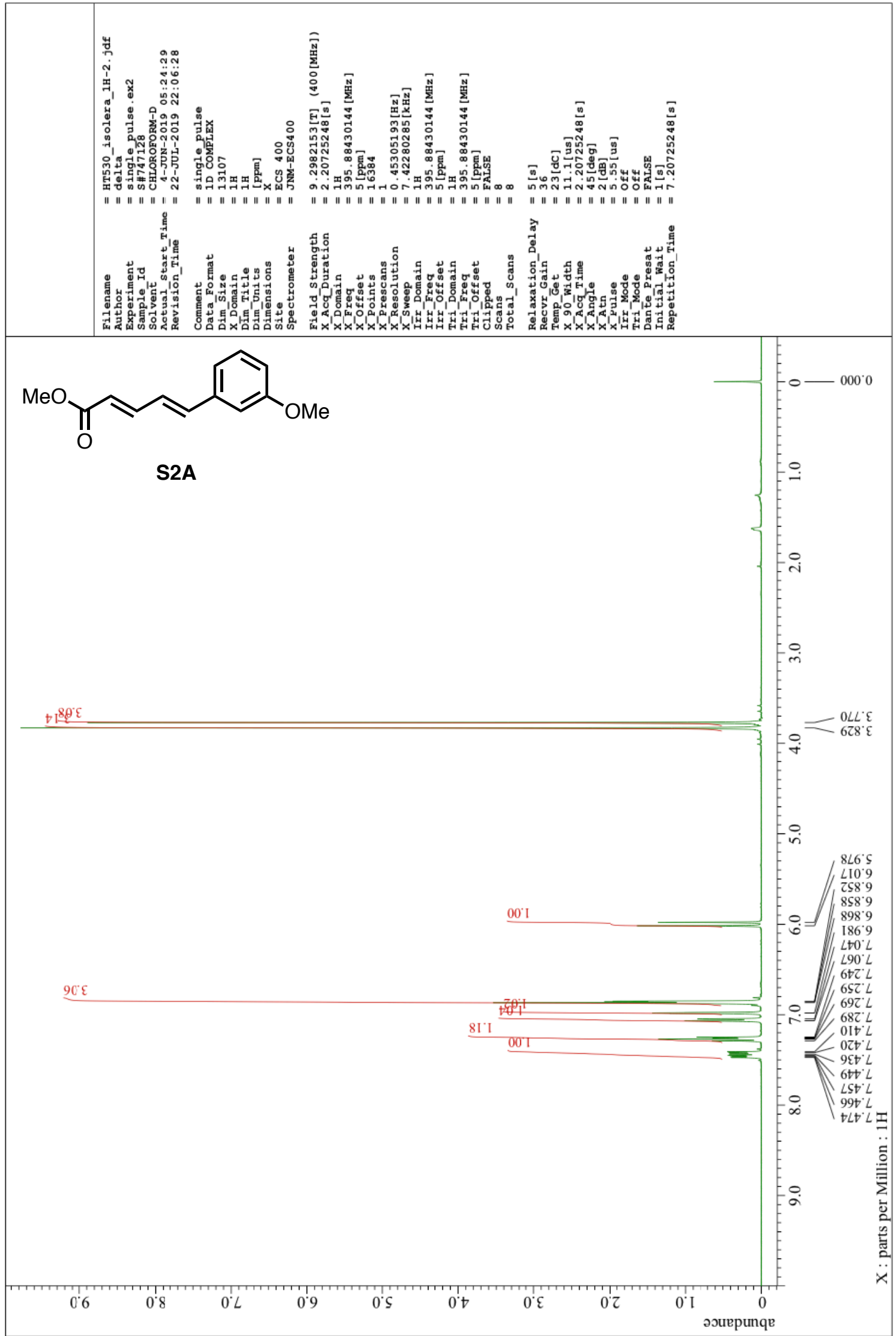


${ }^{13} \mathrm{C}$ NMR of $\mathbf{S 2 A}\left(101 \mathrm{MHz}, \mathrm{CDCl}_{3}\right)$

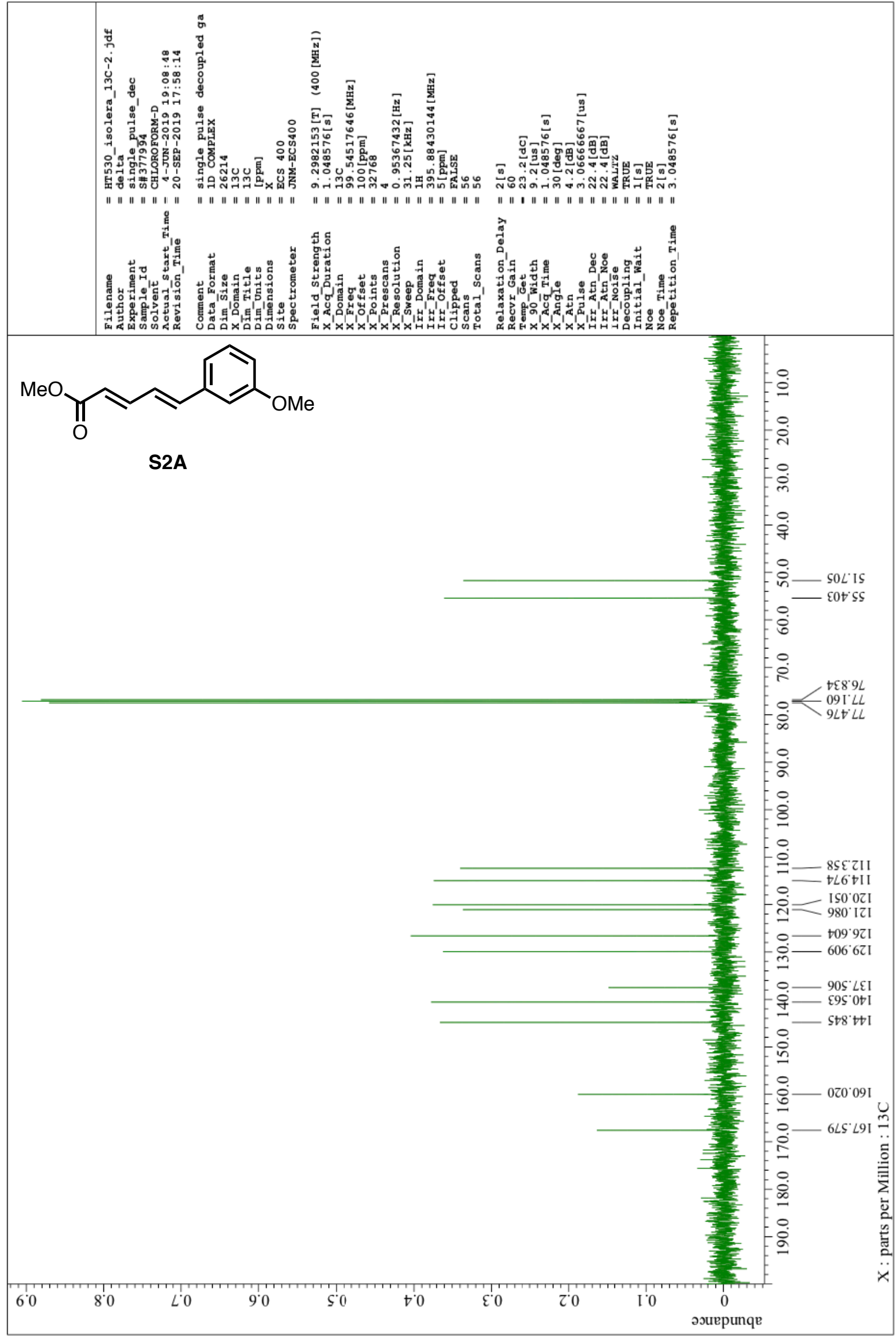


${ }^{1} \mathrm{H}$ NMR of $\mathbf{S 2 C}\left(400 \mathrm{MHz}, \mathrm{CDCl}_{3}\right)$

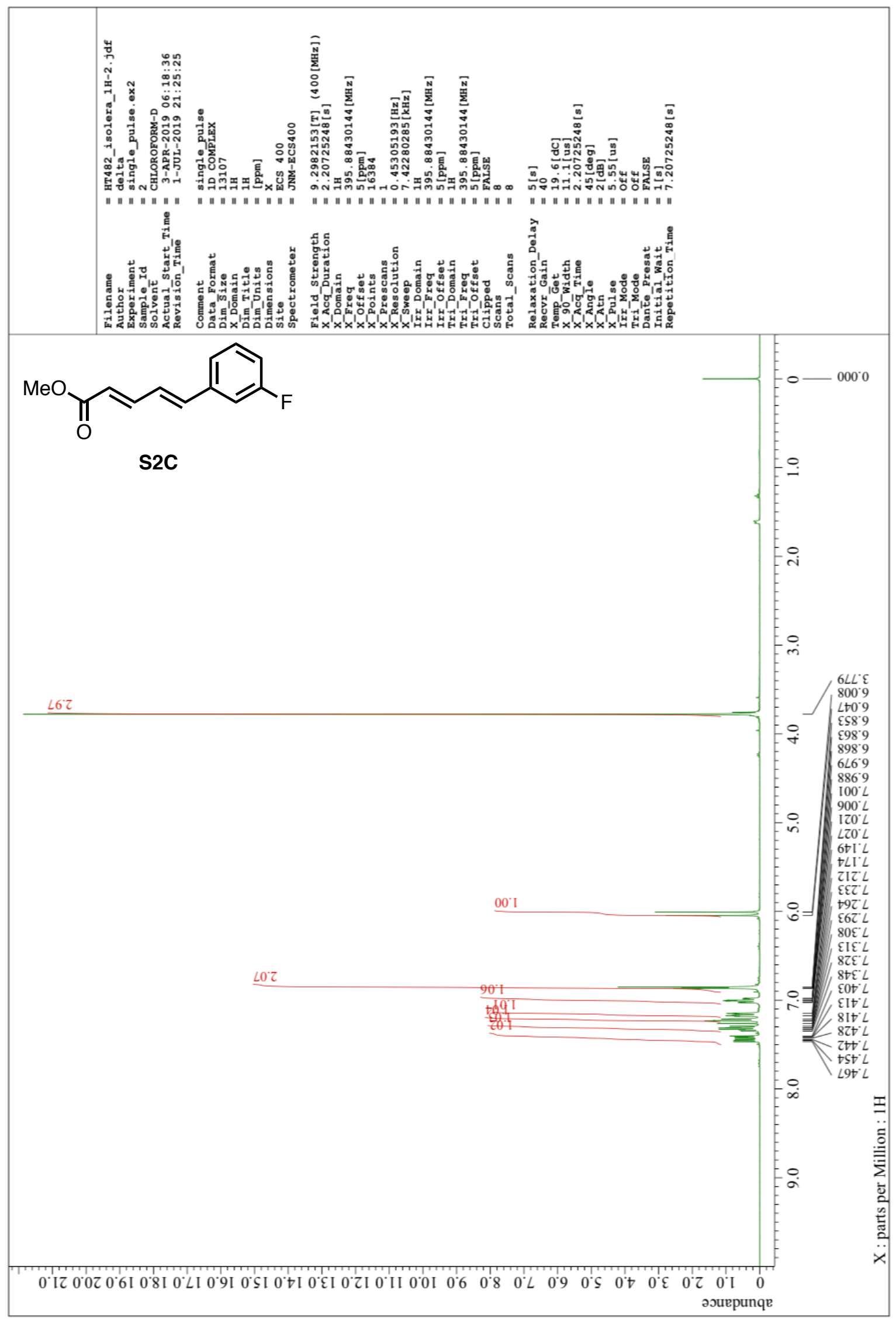


${ }^{13} \mathrm{C}$ NMR of $\mathbf{S 2 C}\left(101 \mathrm{MHz}, \mathrm{CDCl}_{3}\right)$

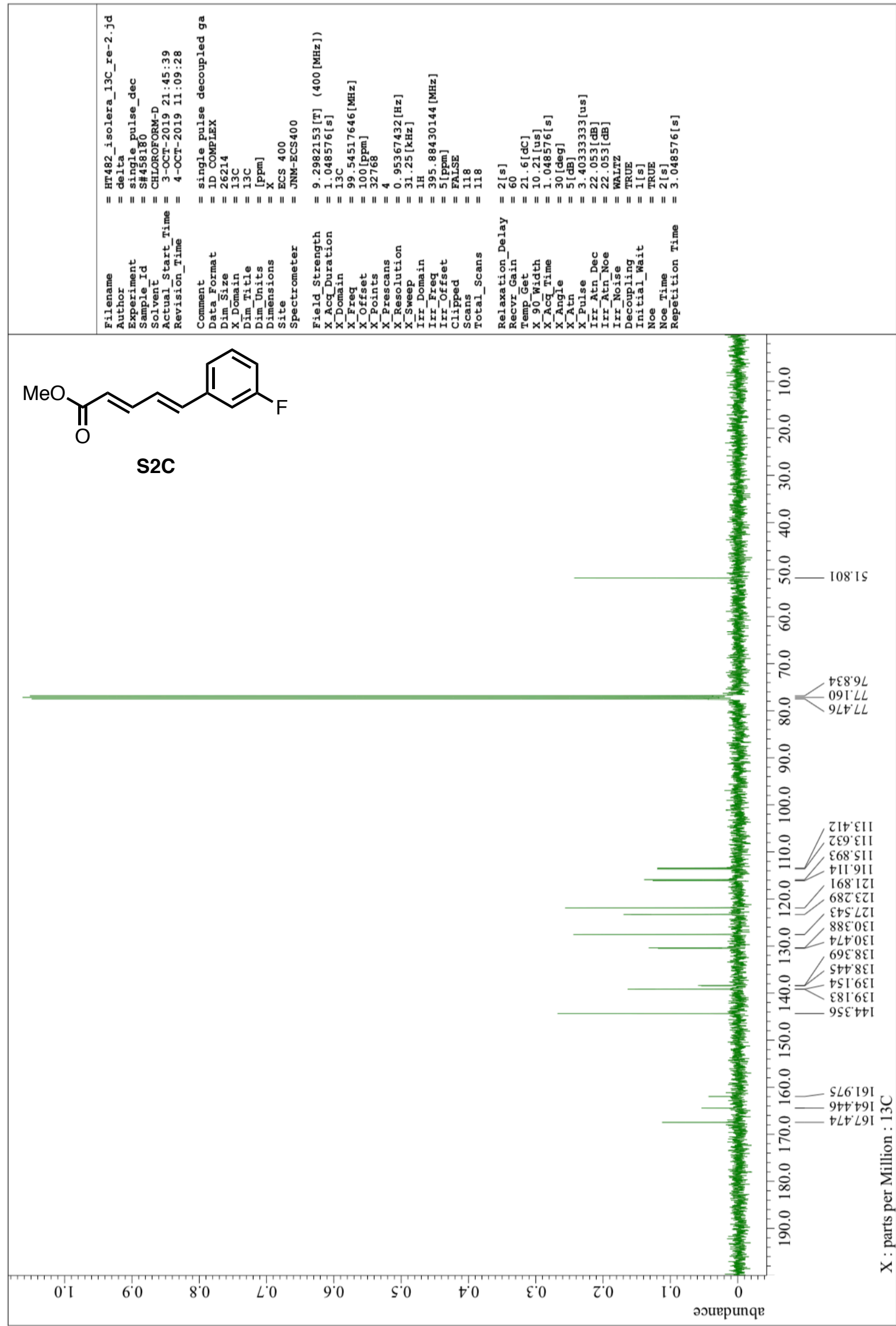


${ }^{1} \mathrm{H}$ NMR of $\mathbf{S 2 E}\left(400 \mathrm{MHz}, \mathrm{CDCl}_{3}\right)$

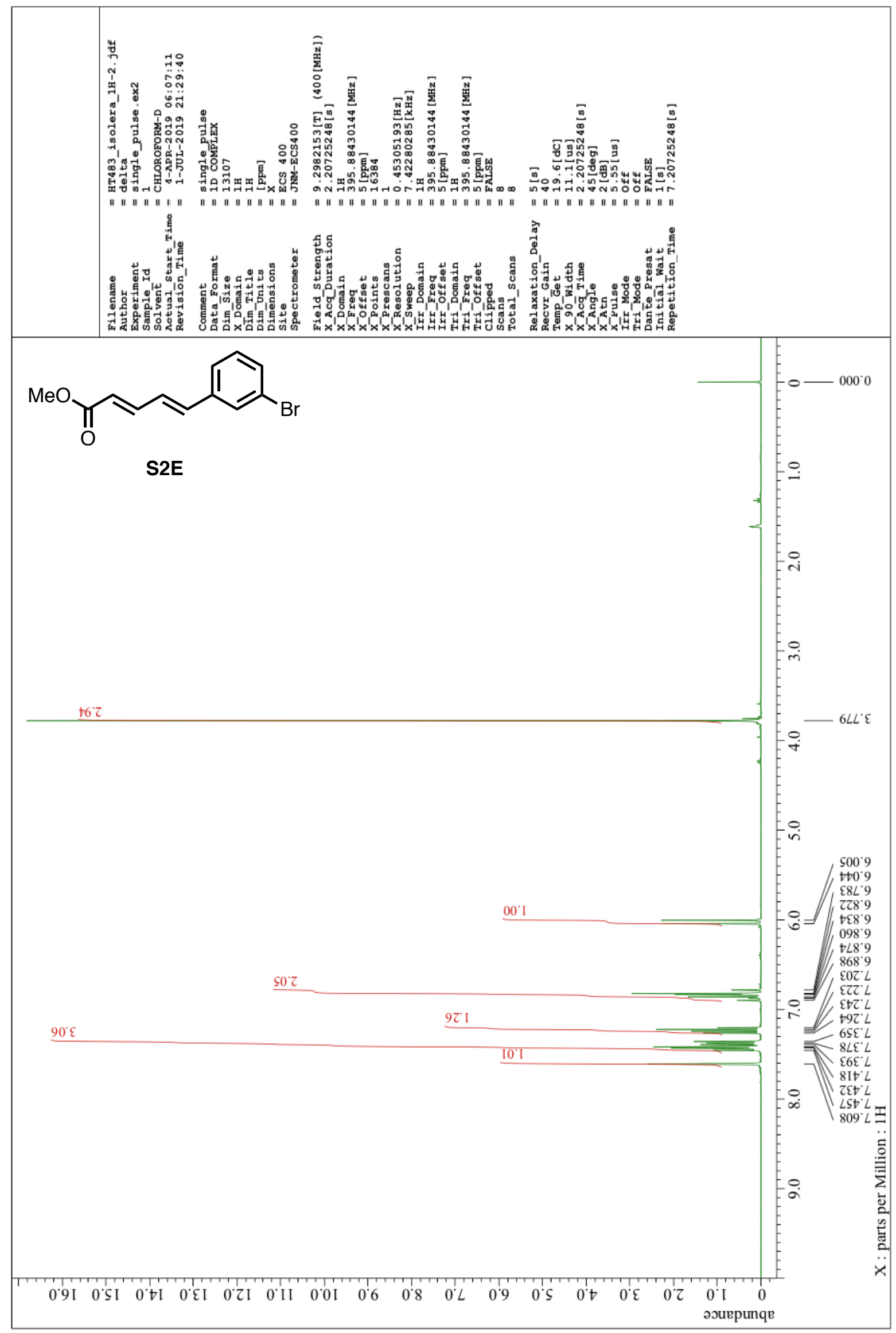


${ }^{13} \mathrm{C}$ NMR of $\mathbf{S 2 E}\left(101 \mathrm{MHz}, \mathrm{CDCl}_{3}\right)$

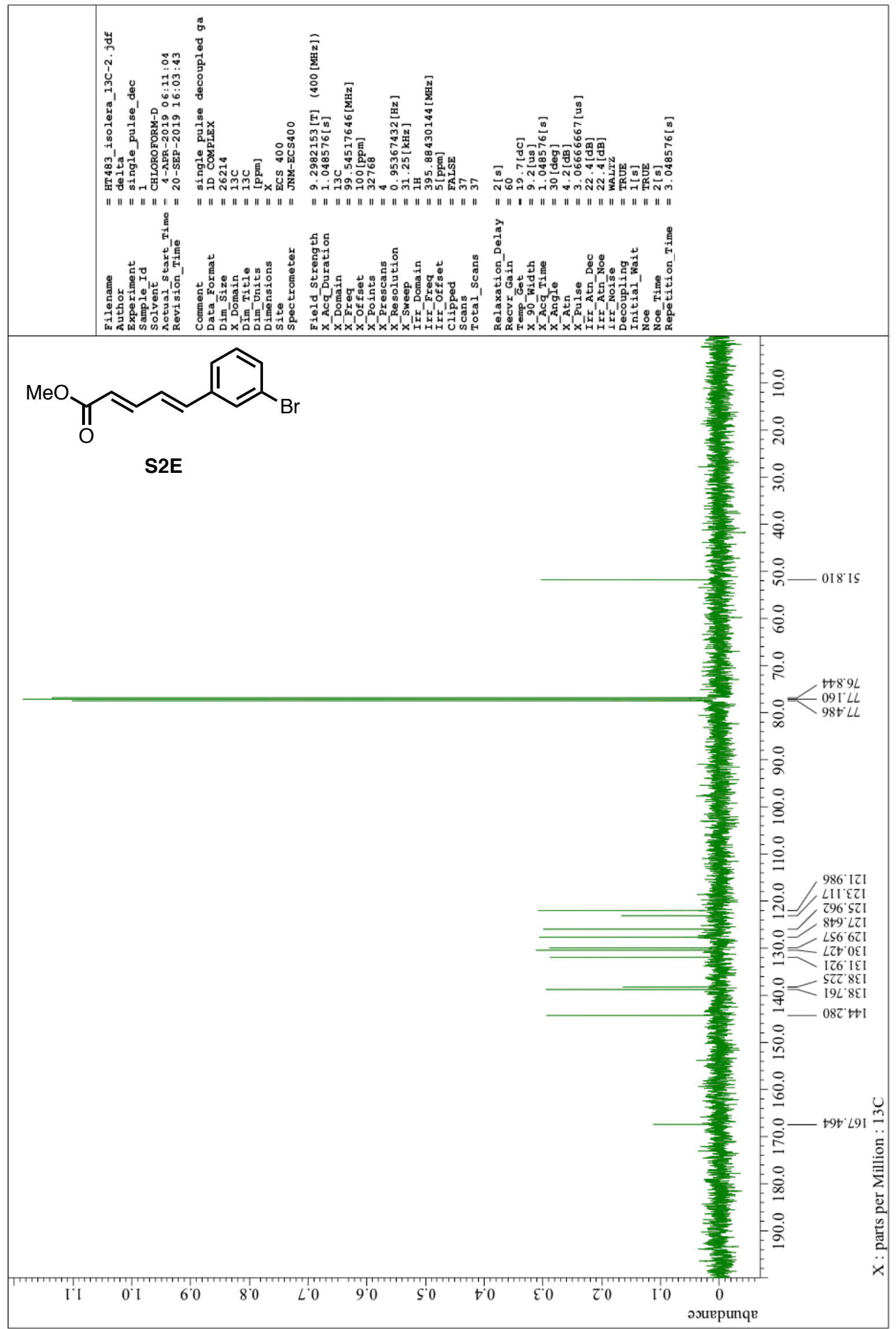


${ }^{1} \mathrm{H}$ NMR of $\mathbf{S 2 F}\left(400 \mathrm{MHz}, \mathrm{CDCl}_{3}\right)$

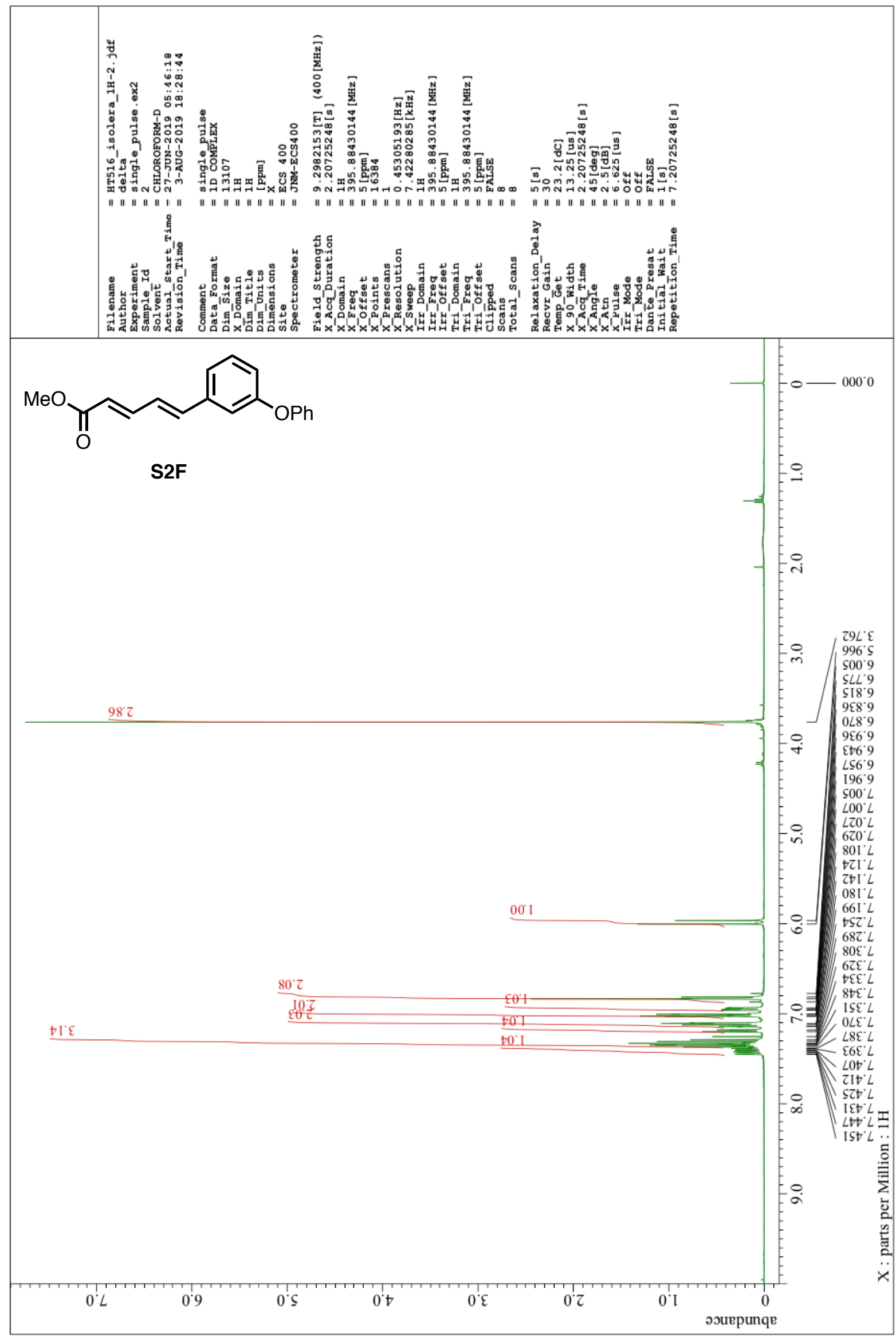


${ }^{13} \mathrm{C}$ NMR of $\mathbf{S 2 F}\left(101 \mathrm{MHz}, \mathrm{CDCl}_{3}\right)$

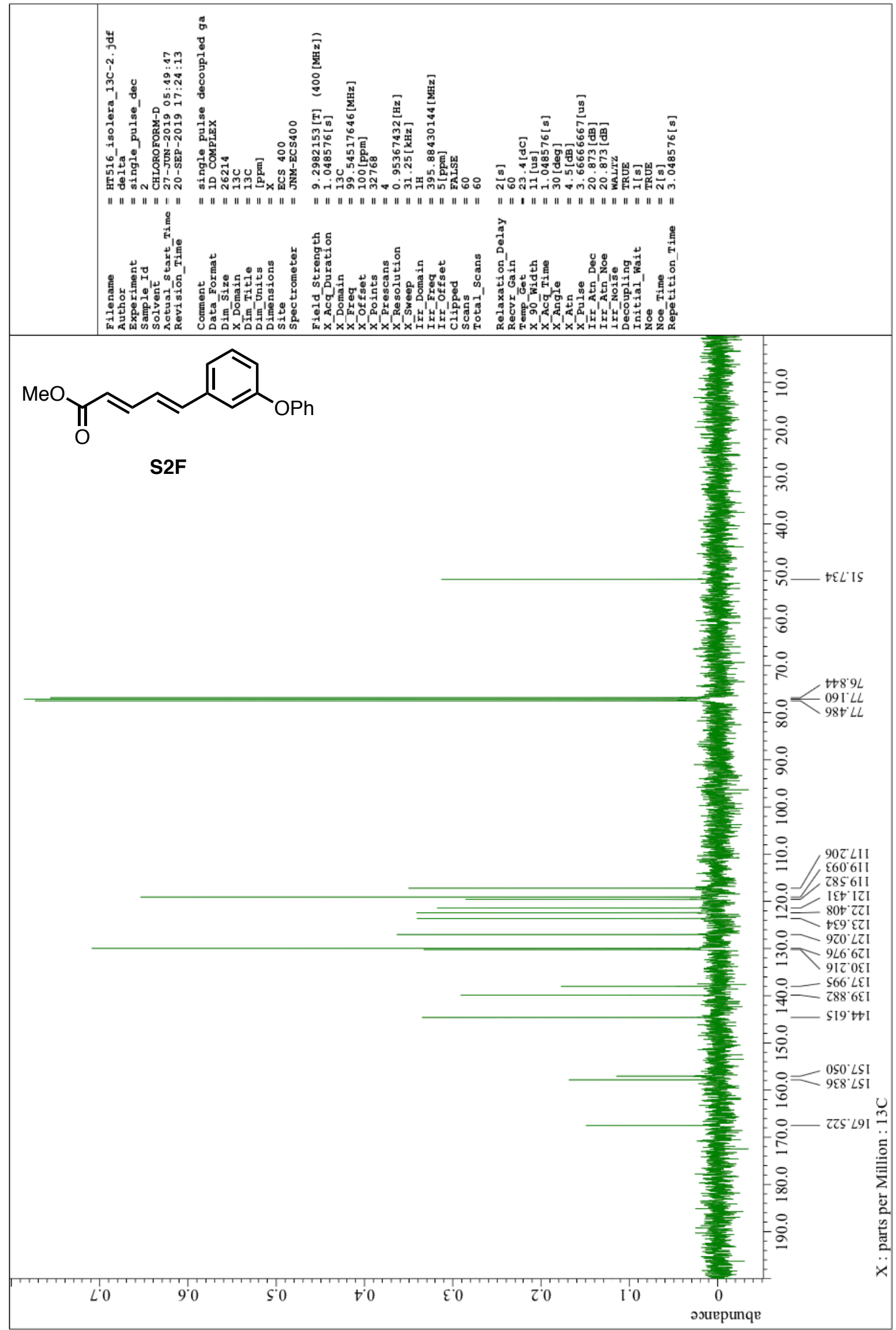


${ }^{1} \mathrm{H}$ NMR of S3A $\left(400 \mathrm{MHz}, \mathrm{CDCl}_{3}\right)$

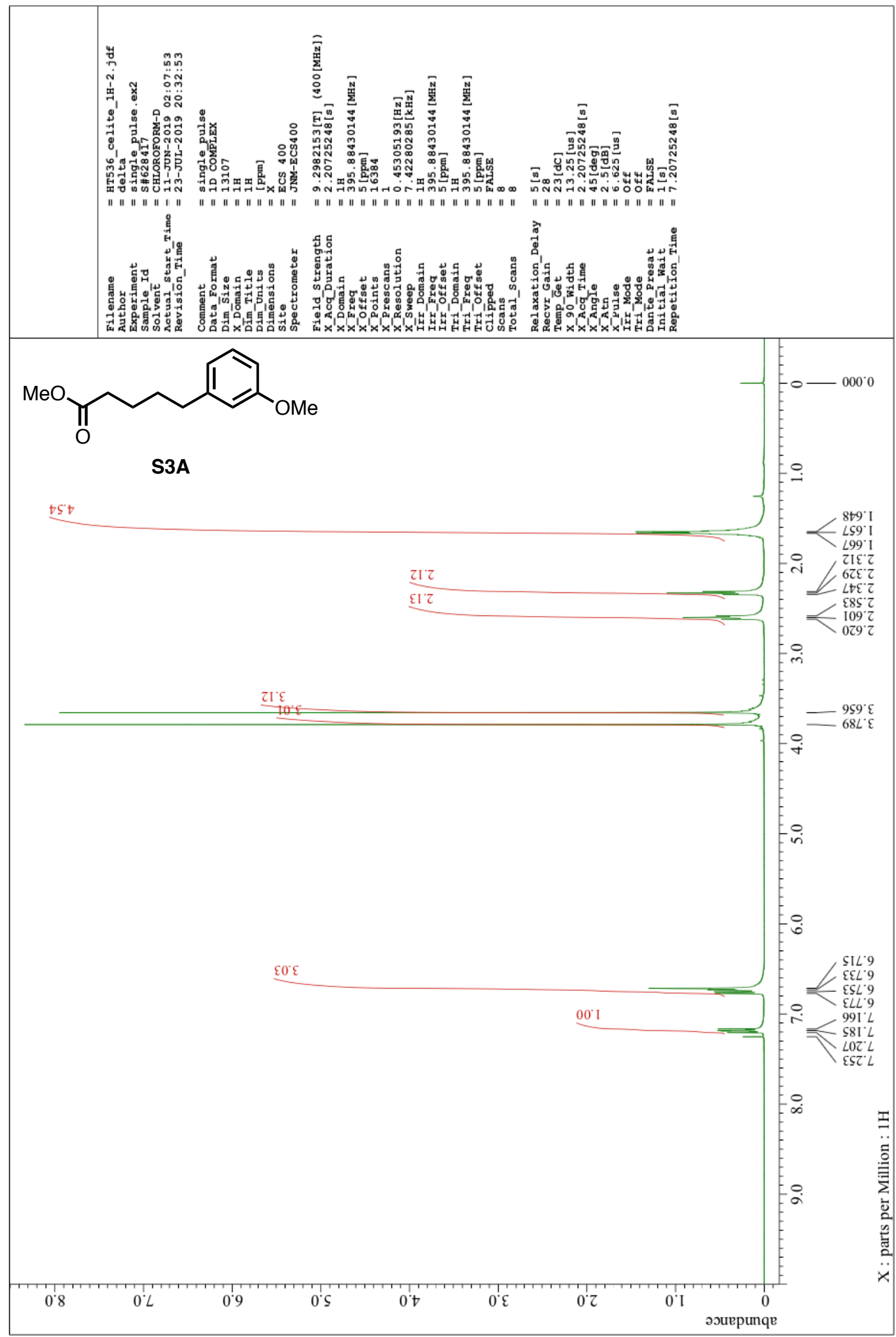


${ }^{13} \mathrm{C}$ NMR of $\mathbf{S 3 A}\left(101 \mathrm{MHz}, \mathrm{CDCl}_{3}\right)$

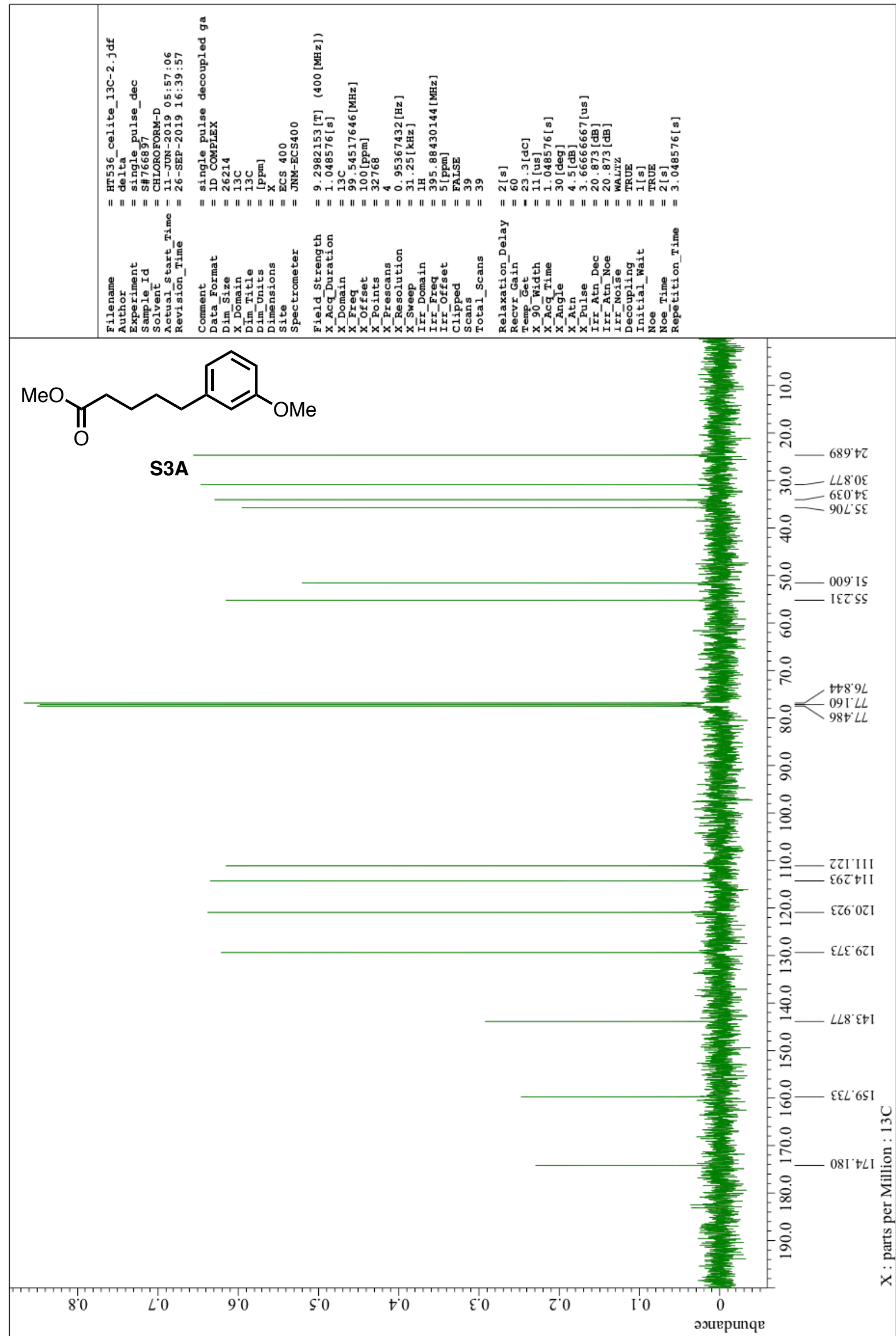


${ }^{1} \mathrm{H}$ NMR of S3B (400 MHz, $\mathrm{CDCl}_{3}$ )

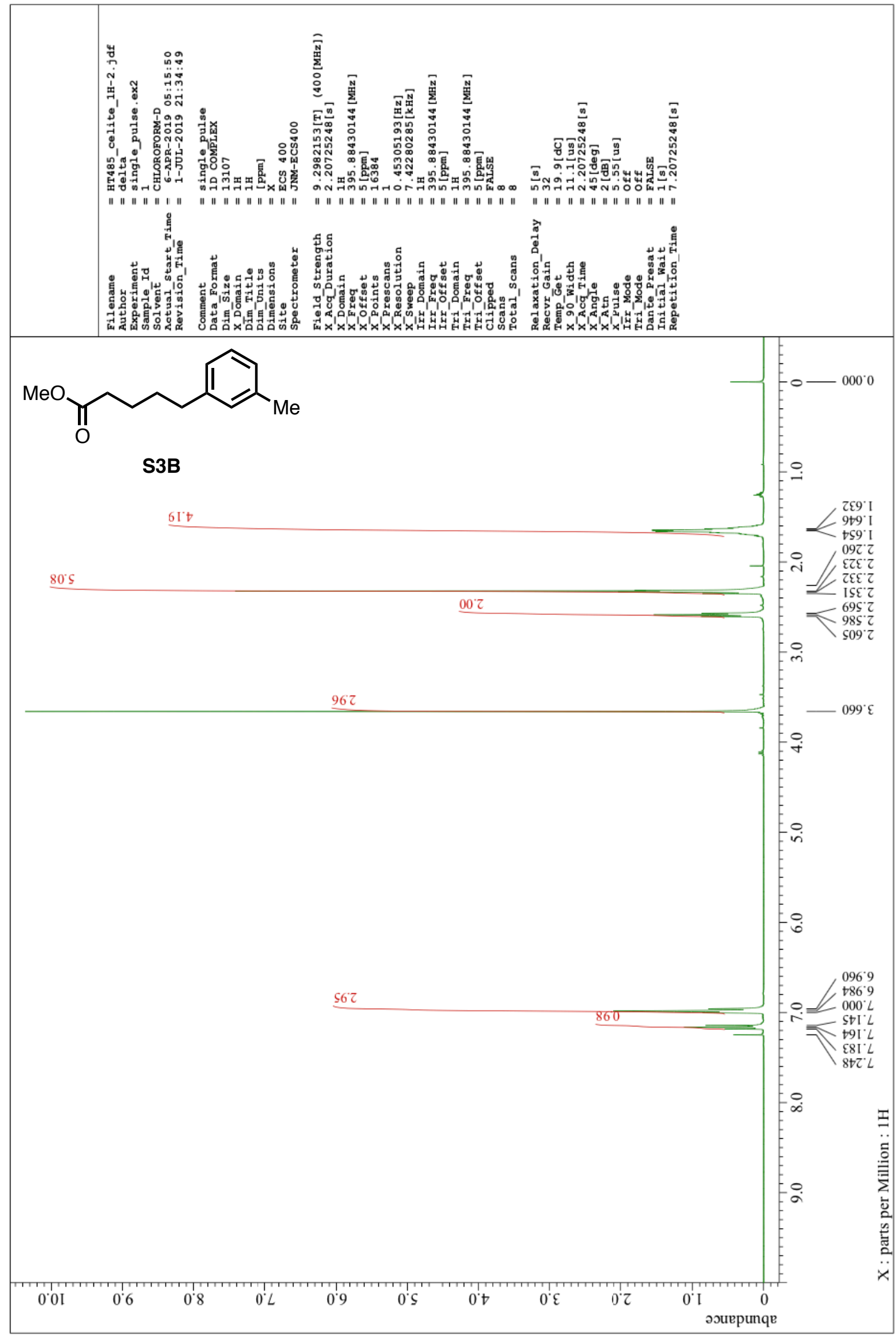


${ }^{13} \mathrm{C}$ NMR of $\mathbf{S 3 B}\left(101 \mathrm{MHz}, \mathrm{CDCl}_{3}\right)$

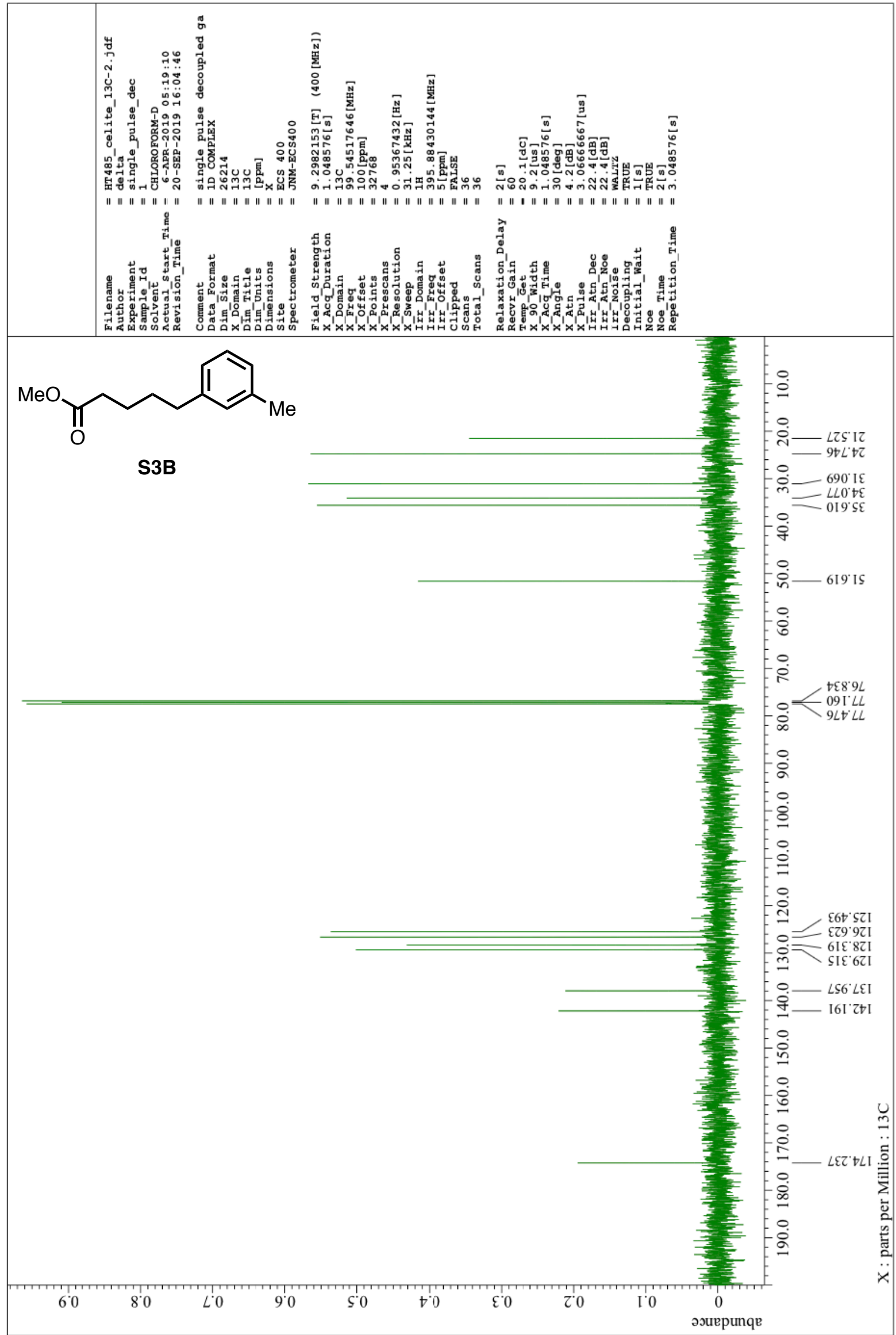


${ }^{1} \mathrm{H}$ NMR of $\mathbf{S 3 C}\left(400 \mathrm{MHz}, \mathrm{CDCl}_{3}\right)$

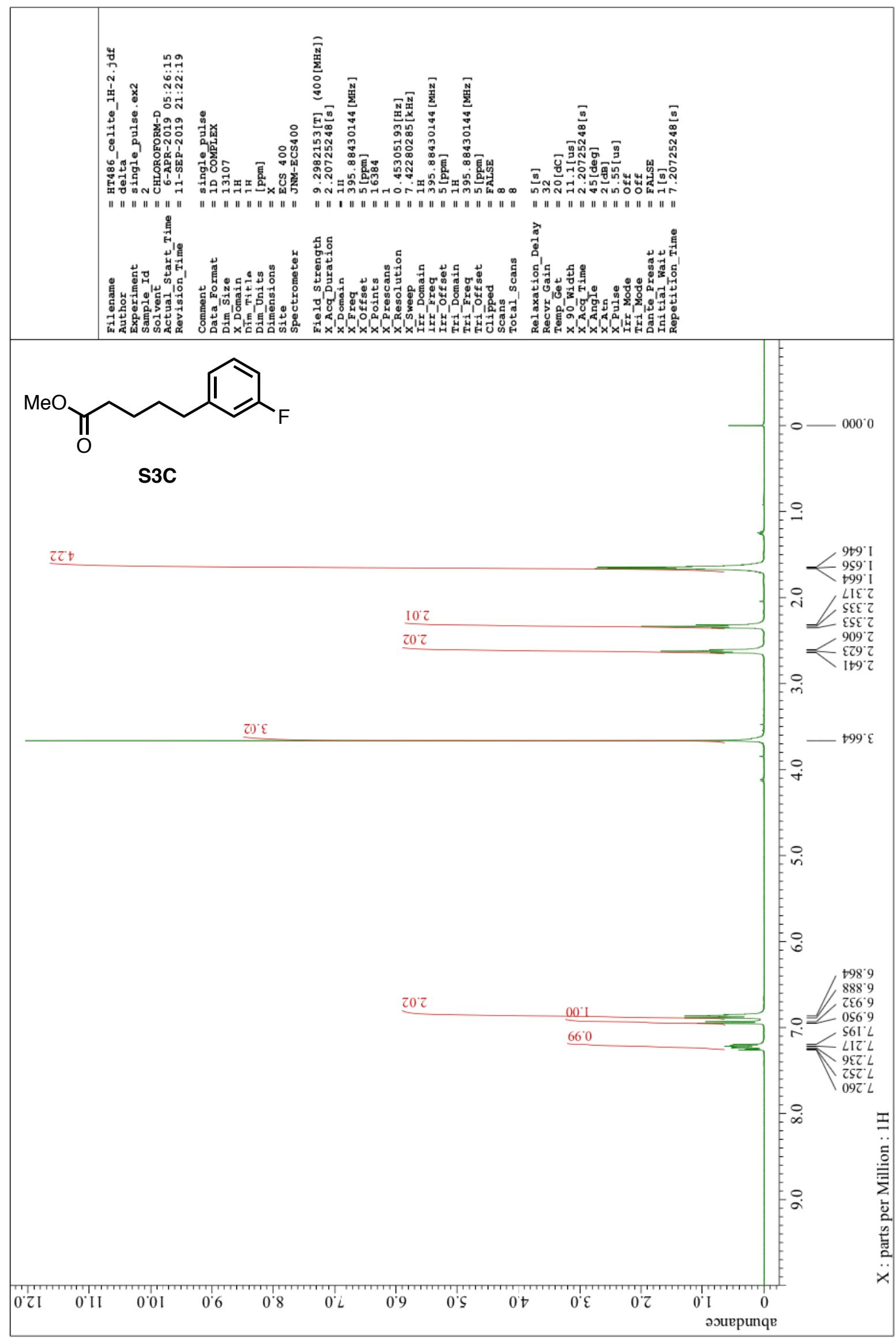


${ }^{13} \mathrm{C}$ NMR of $\mathbf{S 3 C}\left(101 \mathrm{MHz}, \mathrm{CDCl}_{3}\right)$

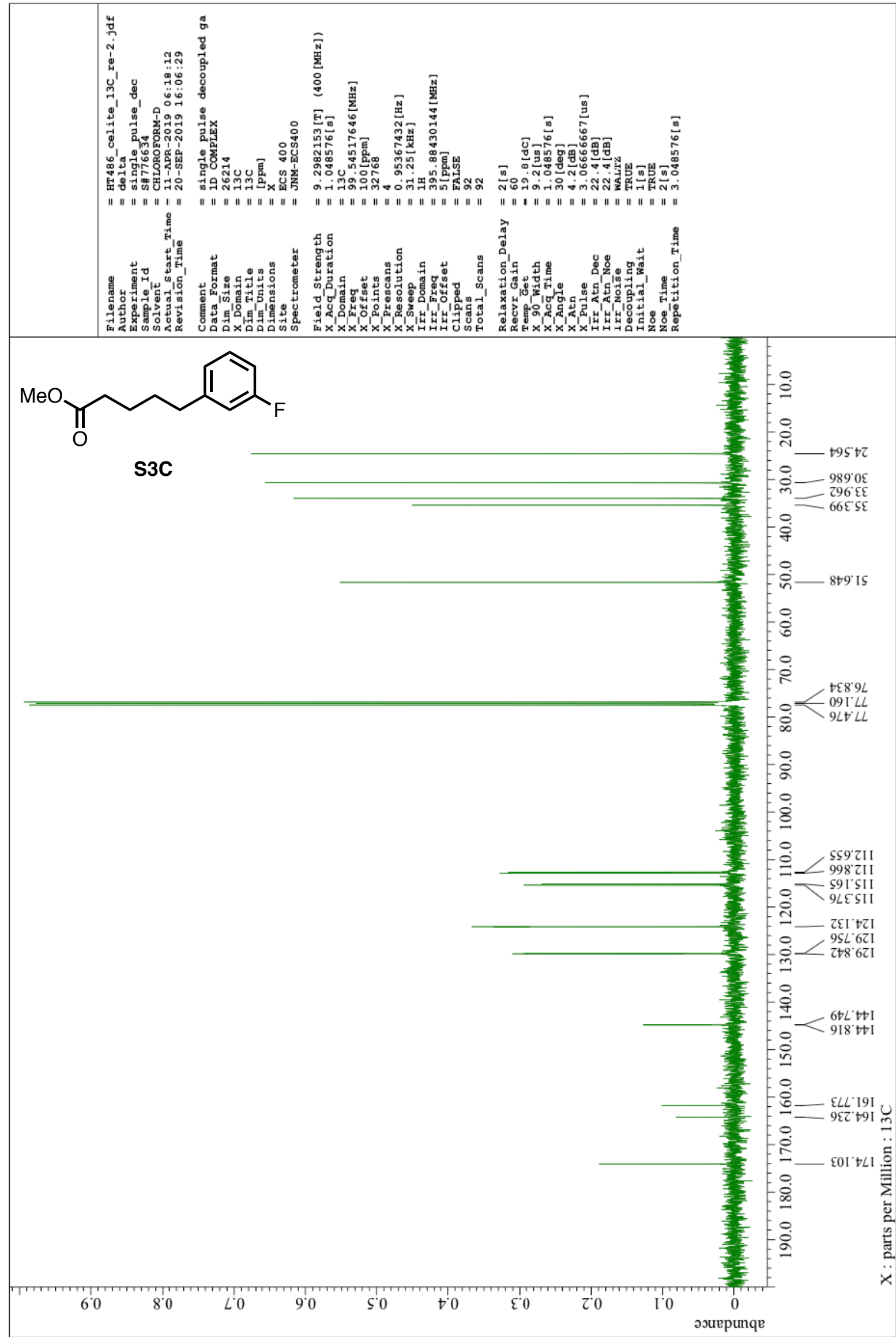


${ }^{1} \mathrm{H}$ NMR of S3D $\left(400 \mathrm{MHz}, \mathrm{CDCl}_{3}\right)$

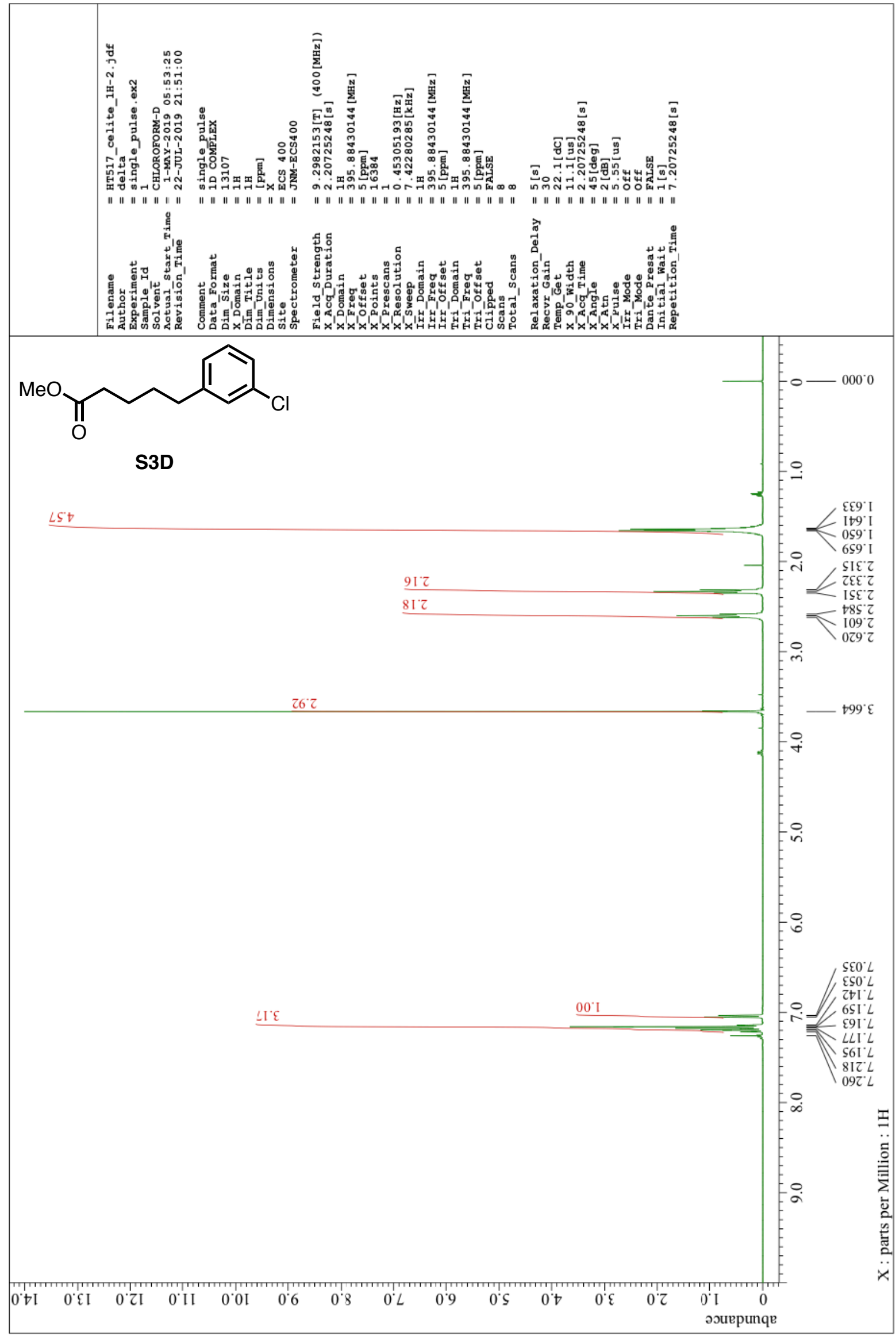


${ }^{13} \mathrm{C}$ NMR of $\mathbf{S 3 D}\left(101 \mathrm{MHz}, \mathrm{CDCl}_{3}\right)$

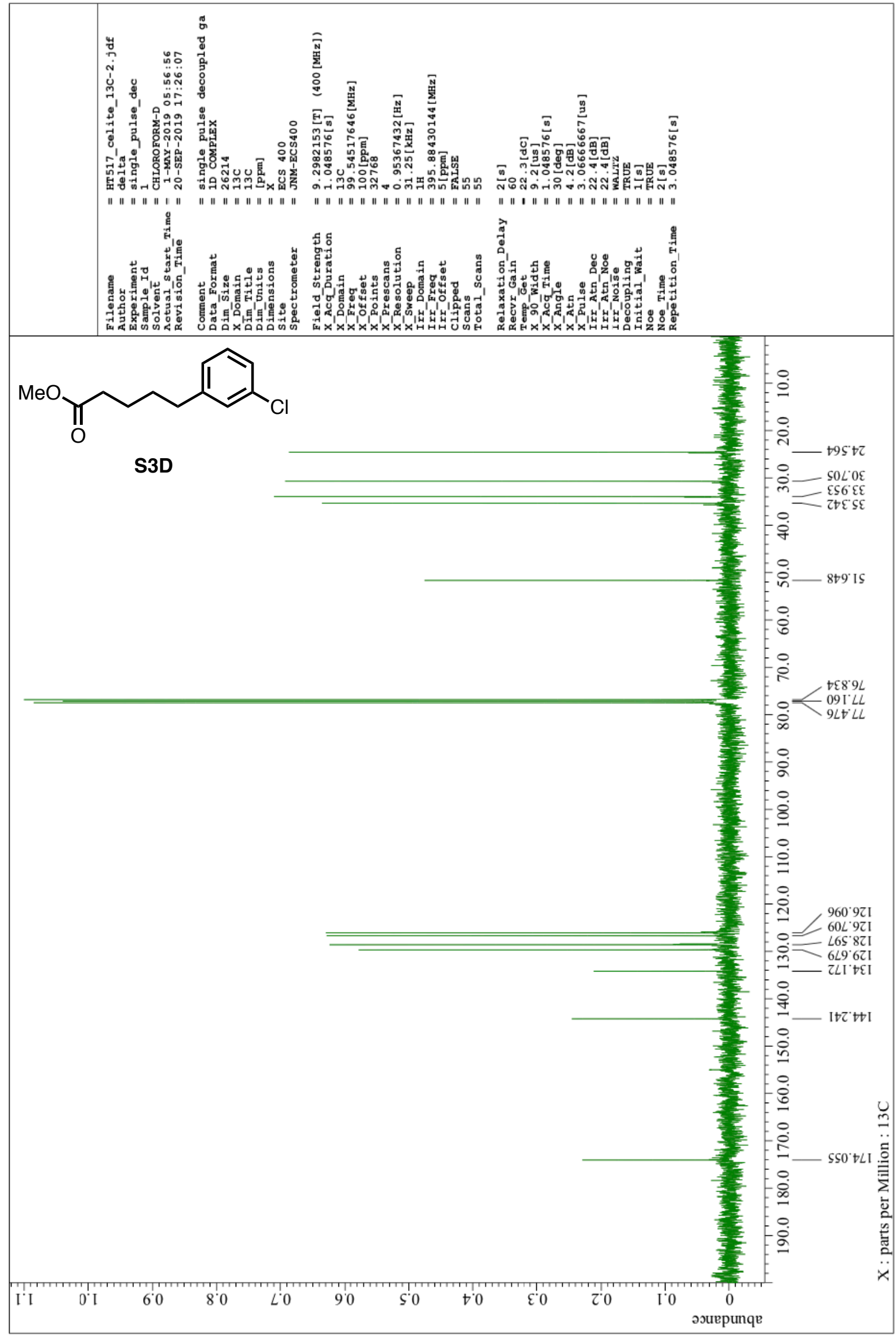


${ }^{1} \mathrm{H}$ NMR of $\mathbf{S 3 E}\left(400 \mathrm{MHz}, \mathrm{CDCl}_{3}\right.$ )

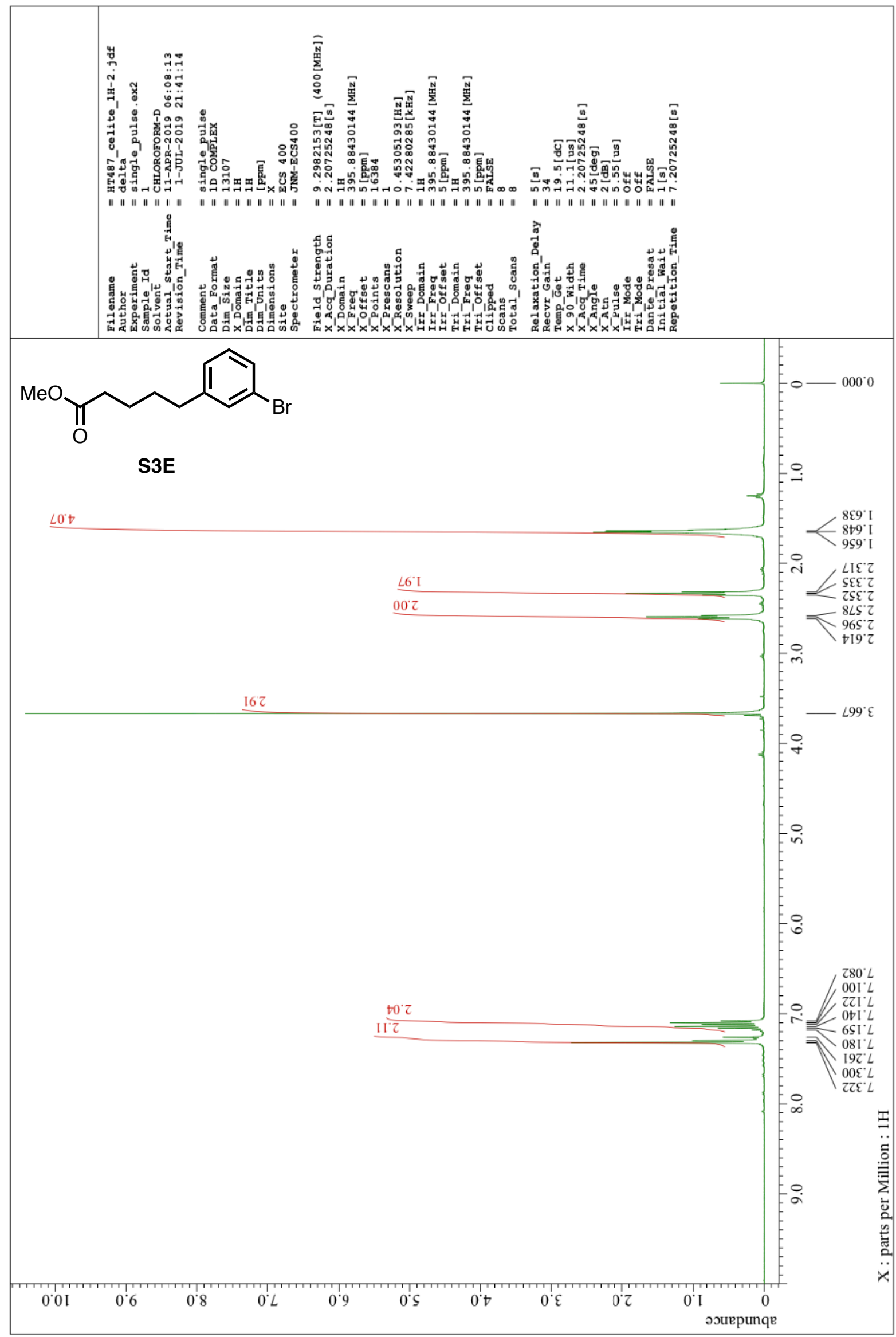


${ }^{13} \mathrm{C}$ NMR of $\mathbf{S 3 E}\left(101 \mathrm{MHz}, \mathrm{CDCl}_{3}\right)$

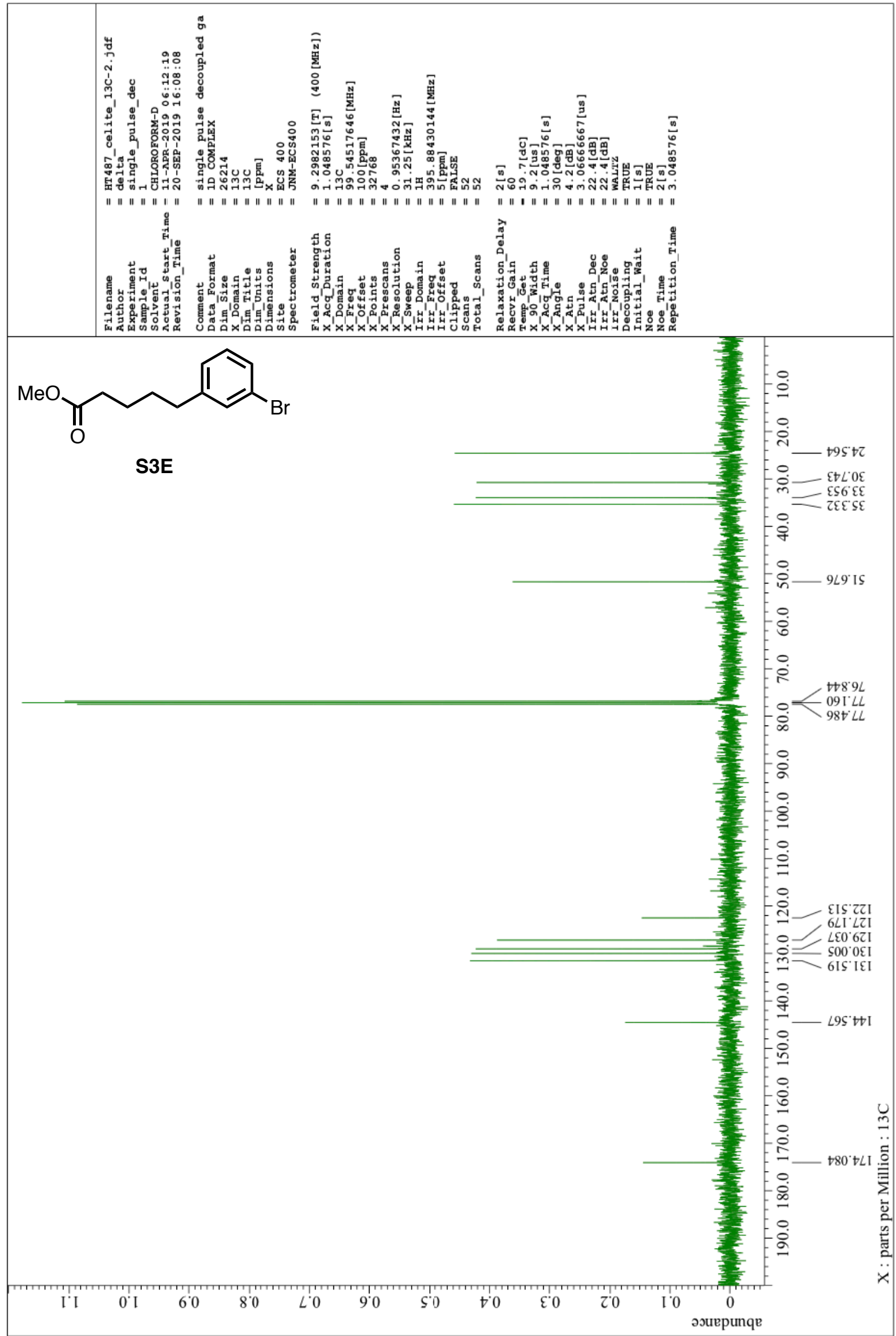


${ }^{1} \mathrm{H}$ NMR of $\mathbf{S 3 F}\left(400 \mathrm{MHz}, \mathrm{CDCl}_{3}\right)$

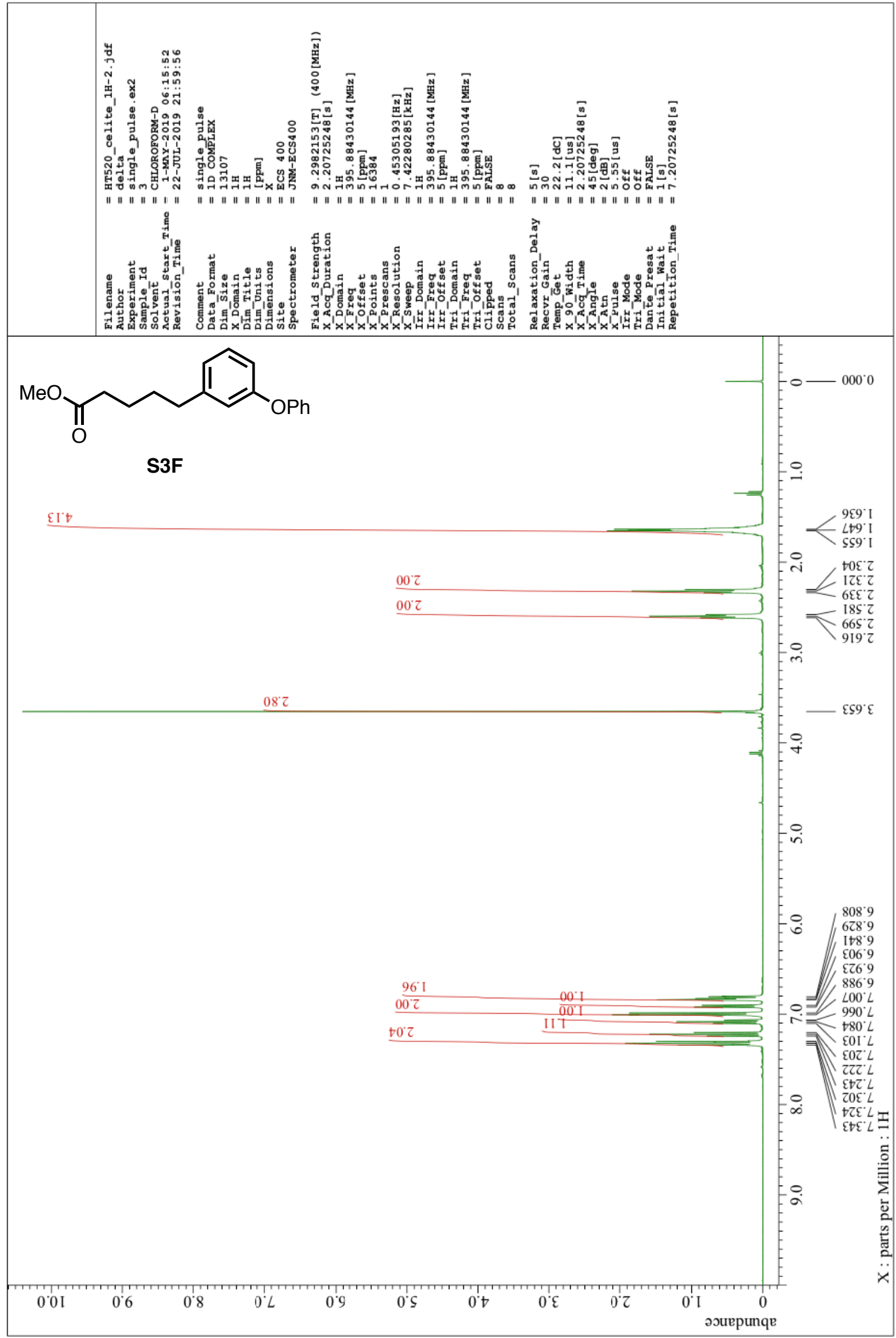


${ }^{13} \mathrm{C}$ NMR of $\mathbf{S 3 F}\left(101 \mathrm{MHz}, \mathrm{CDCl}_{3}\right)$

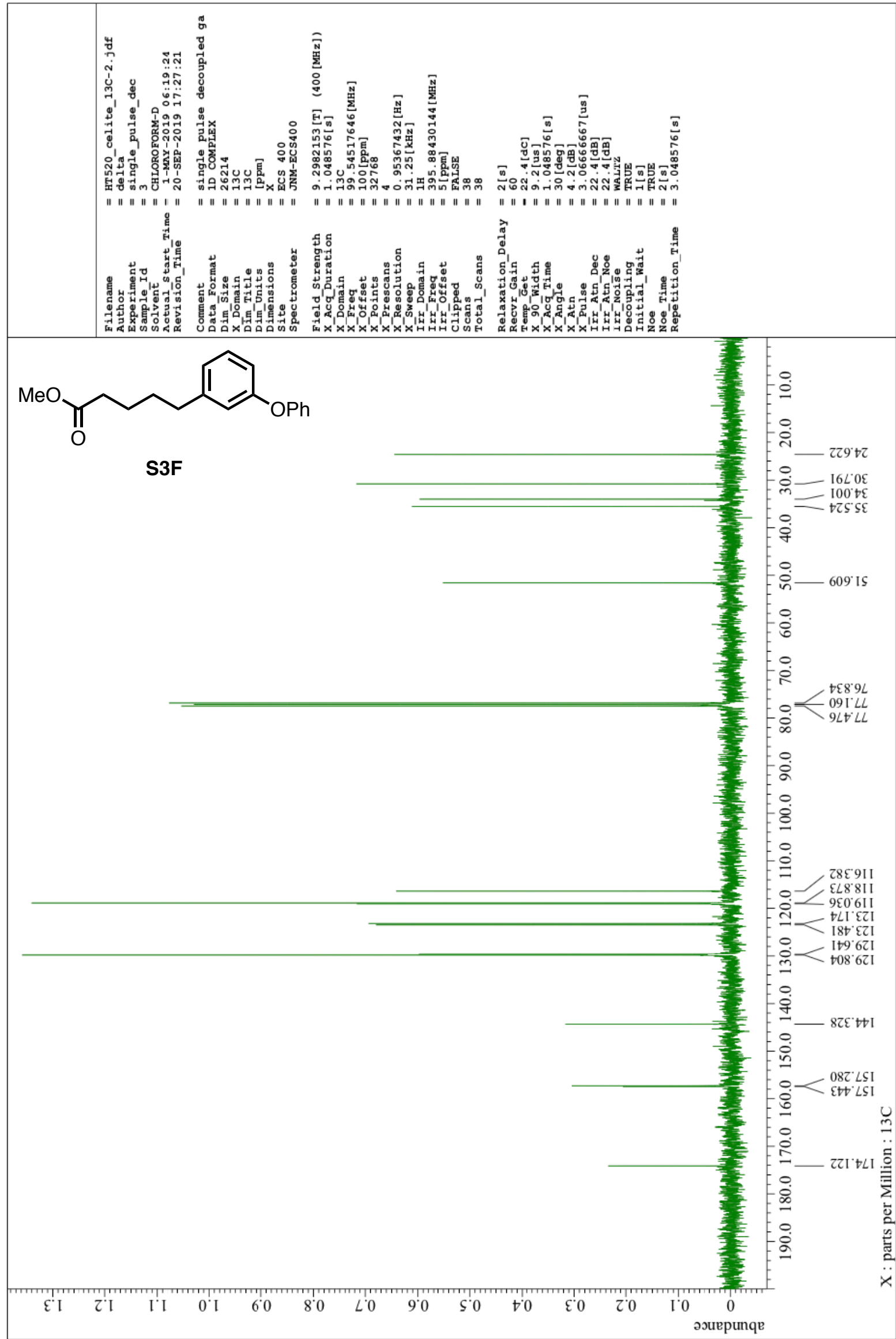


${ }^{1} \mathrm{H}$ NMR of S3G $\left(400 \mathrm{MHz}, \mathrm{CDCl}_{3}\right)$

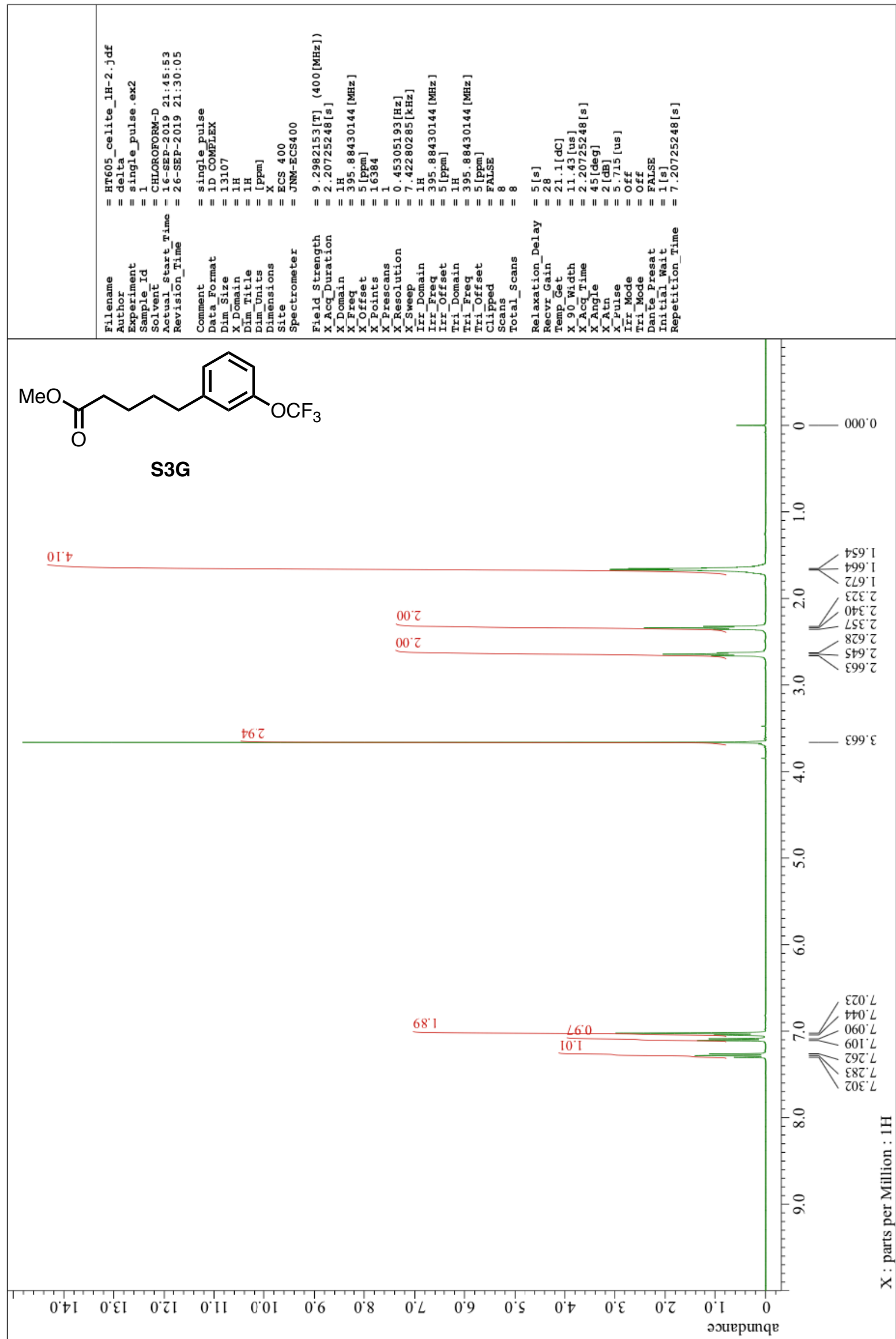


${ }^{13} \mathrm{C}$ NMR of $\mathbf{S 3 G}\left(101 \mathrm{MHz}, \mathrm{CDCl}_{3}\right)$

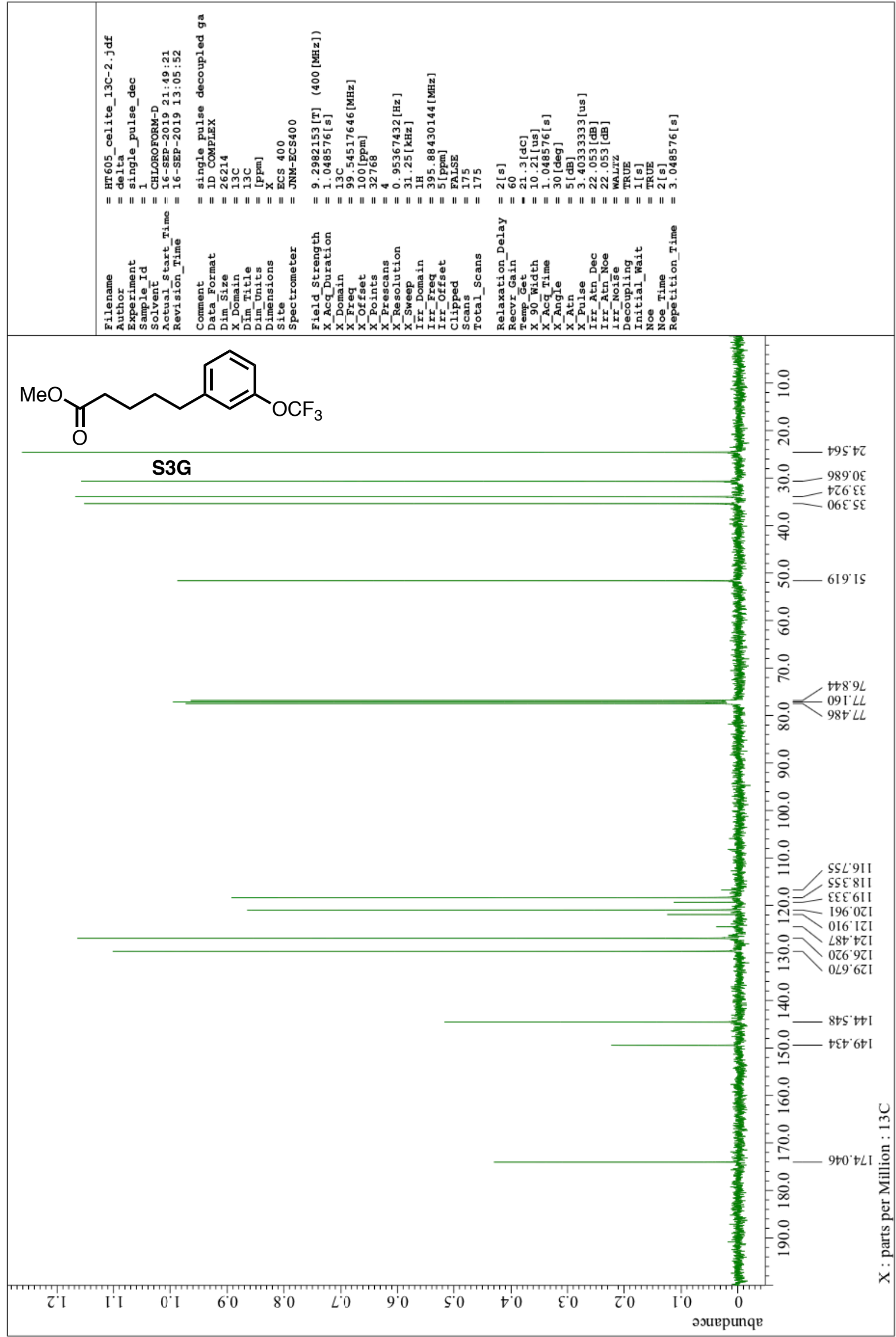


${ }^{1} \mathrm{H}$ NMR of $\mathbf{S 3 H}\left(400 \mathrm{MHz}, \mathrm{CDCl}_{3}\right)$

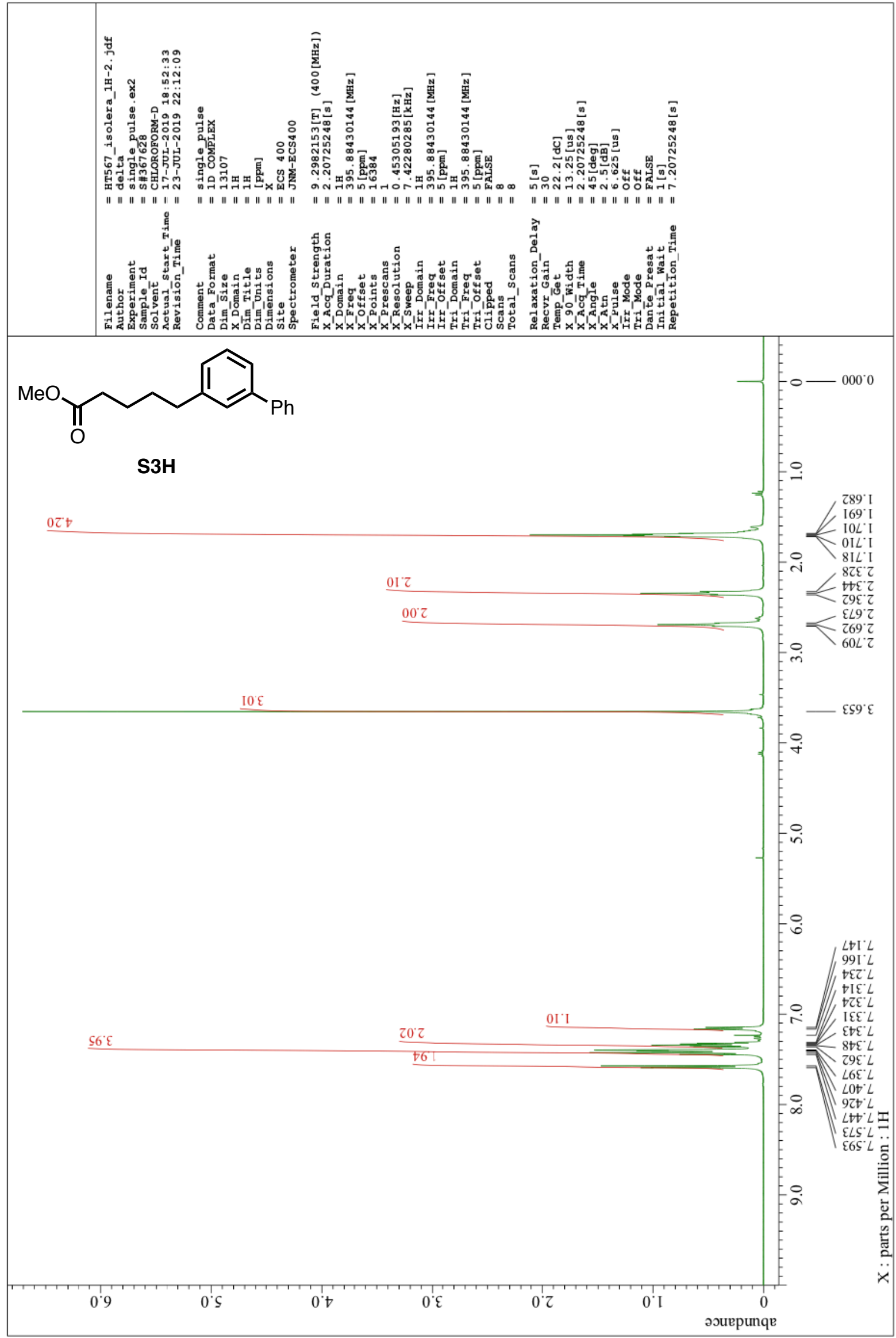


${ }^{13} \mathrm{C}$ NMR of $\mathbf{S 3 H}\left(101 \mathrm{MHz}, \mathrm{CDCl}_{3}\right)$

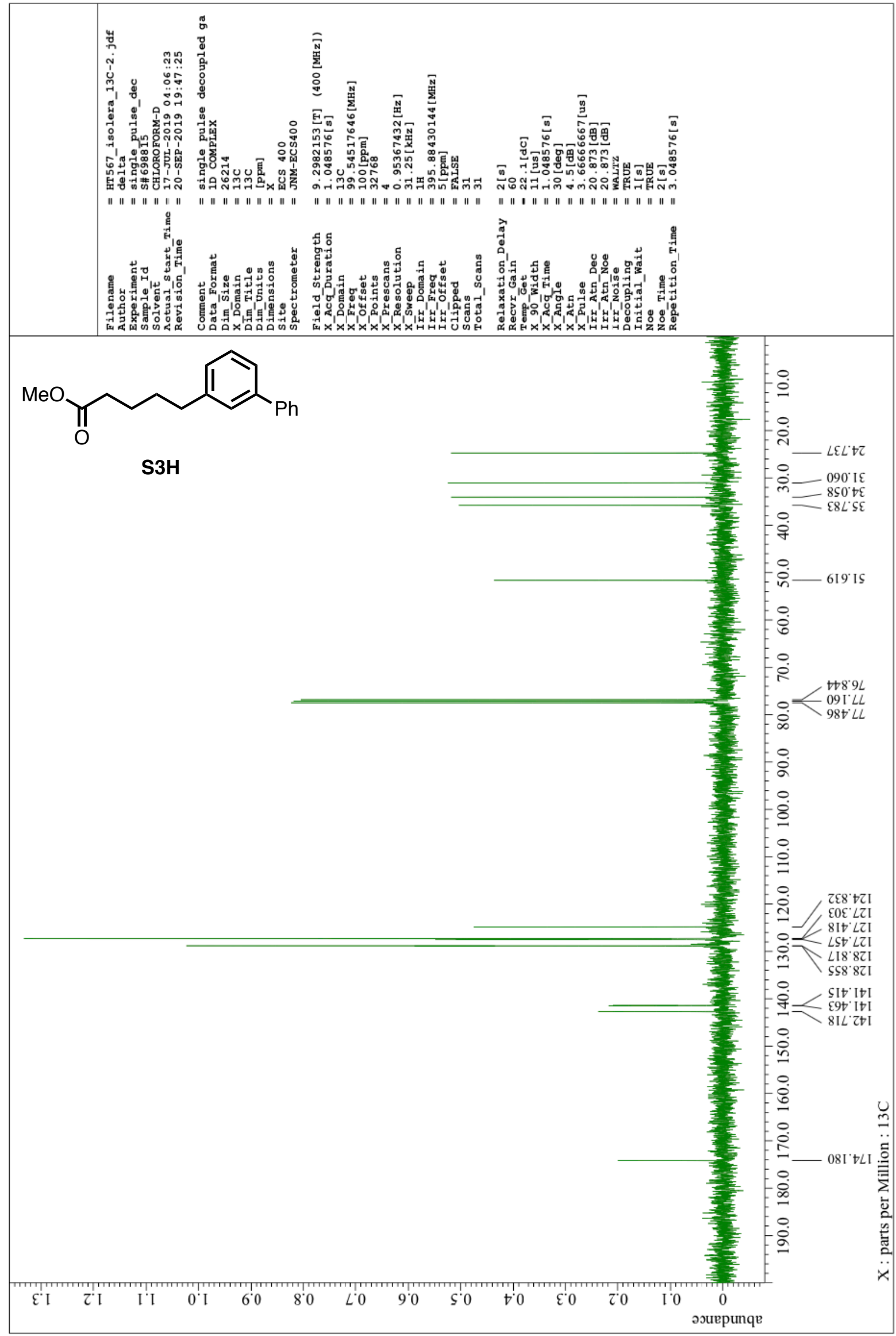


${ }^{1} \mathrm{H}$ NMR of S3I (400 MHz, $\left.\mathrm{CDCl}_{3}\right)$

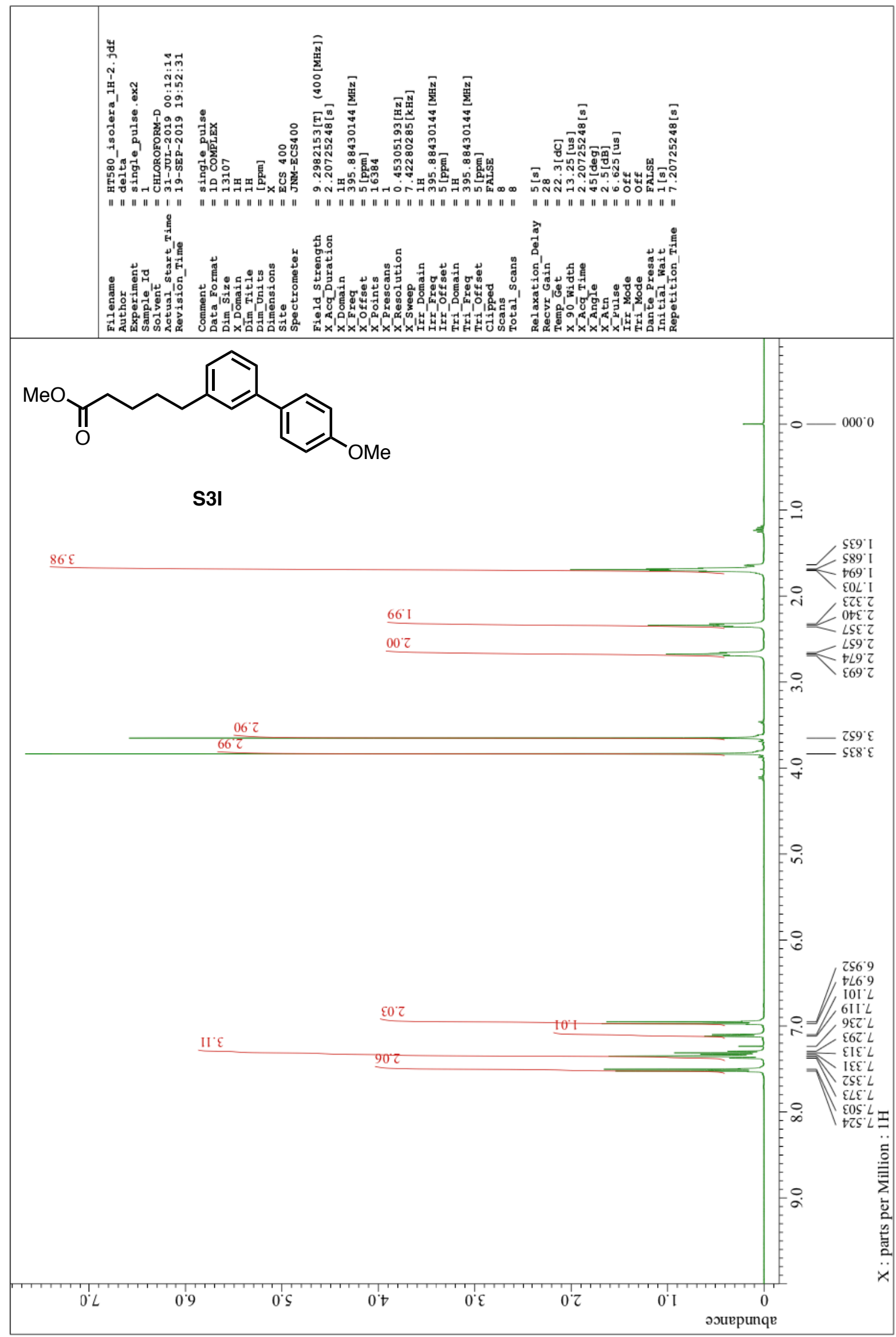


${ }^{13} \mathrm{C}$ NMR of S3I $\left(101 \mathrm{MHz}, \mathrm{CDCl}_{3}\right)$

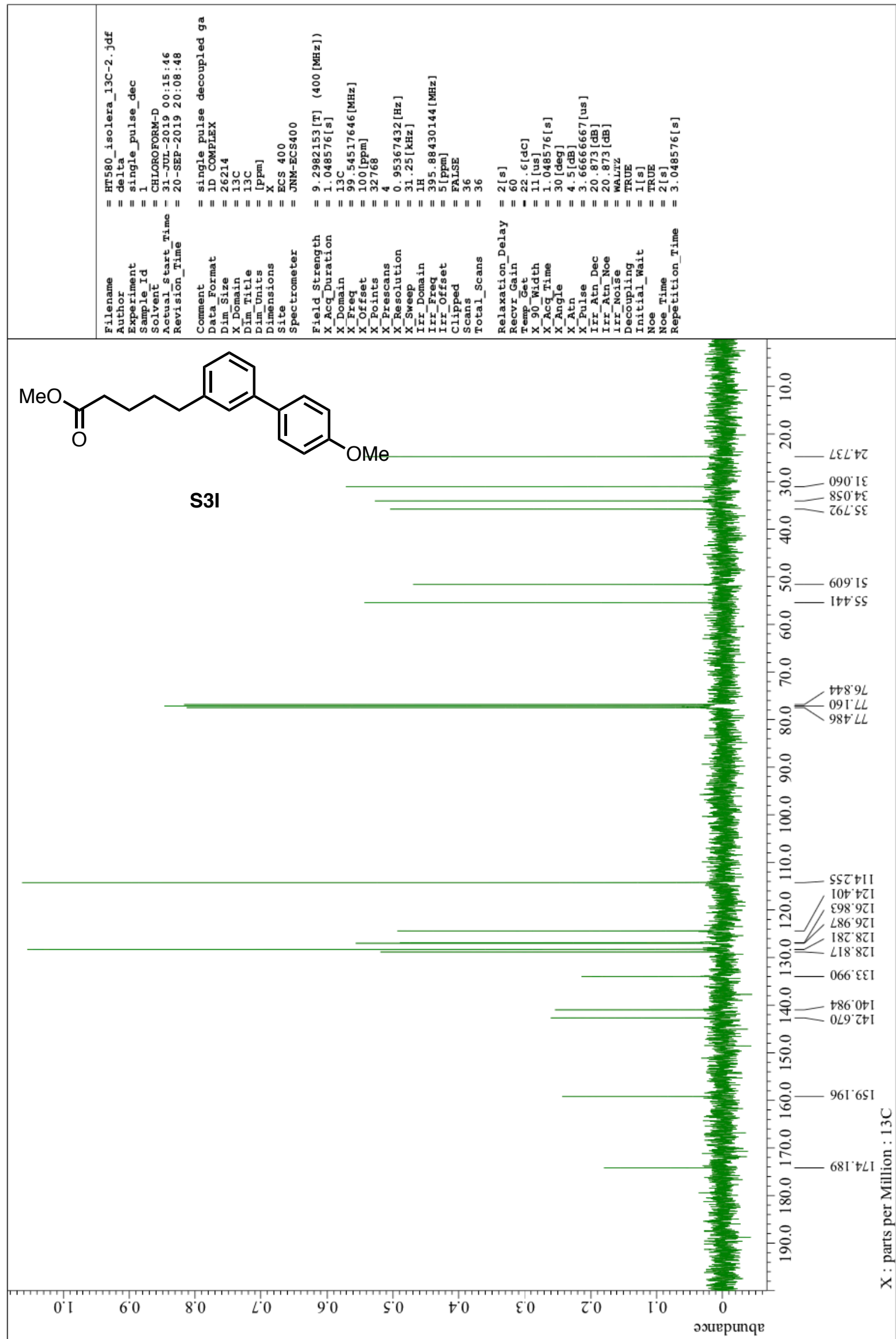


${ }^{1} \mathrm{H}$ NMR of $\mathbf{S 3 J}\left(400 \mathrm{MHz}, \mathrm{CDCl}_{3}\right.$ )

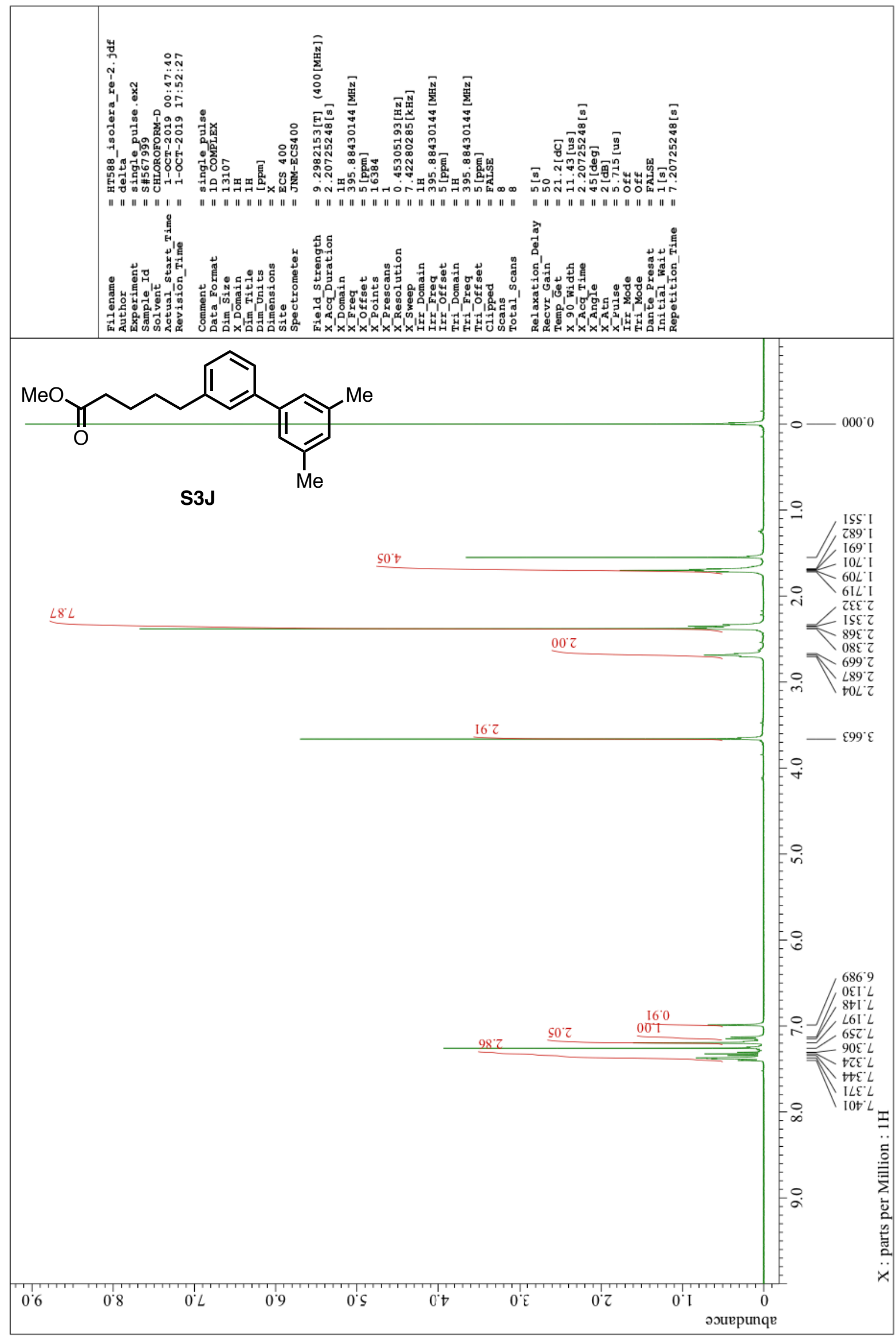


${ }^{13} \mathrm{C}$ NMR of S3J $\left(101 \mathrm{MHz}, \mathrm{CDCl}_{3}\right)$

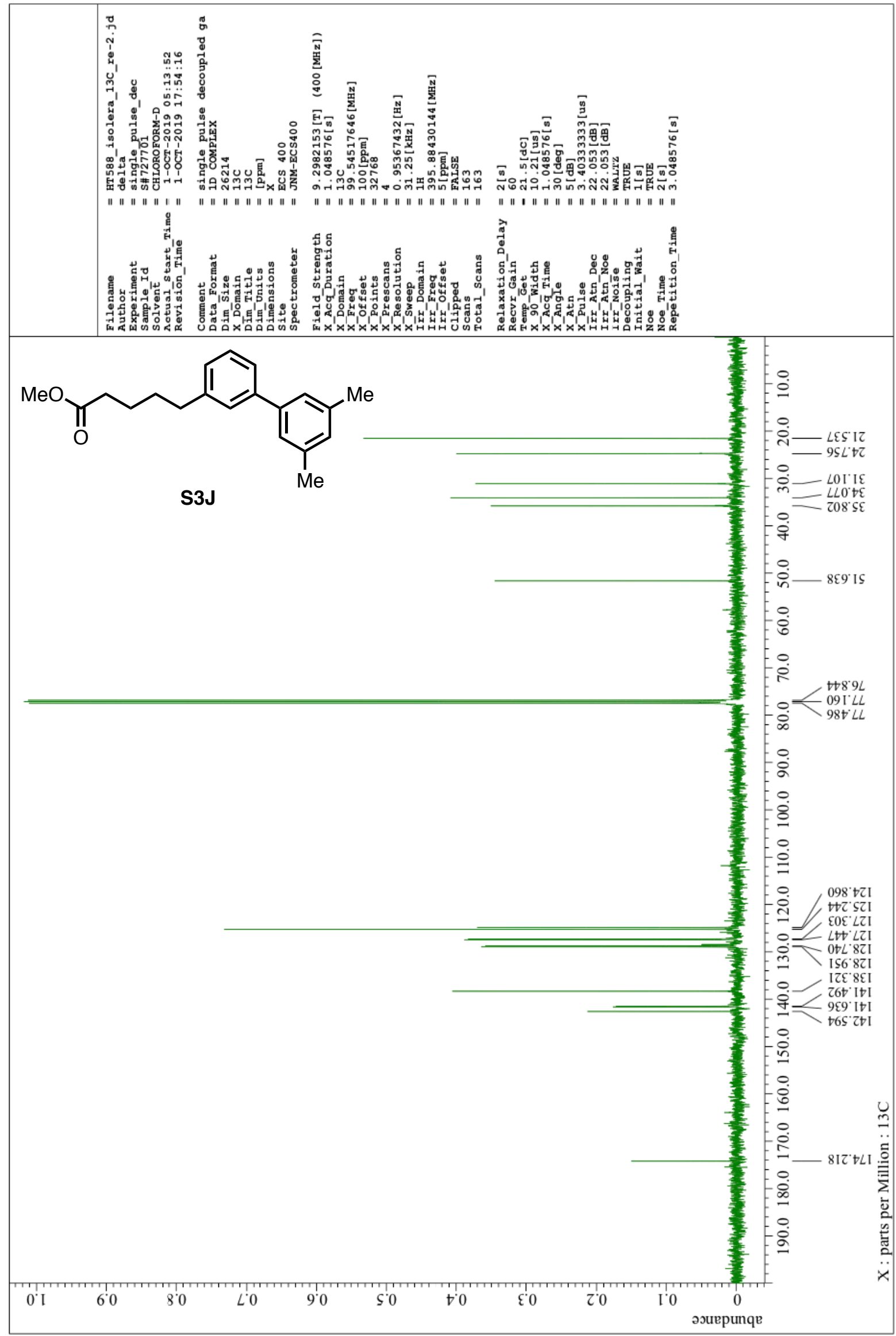


${ }^{1} \mathrm{H}$ NMR of $\mathbf{S 3 K}\left(400 \mathrm{MHz}, \mathrm{CDCl}_{3}\right)$

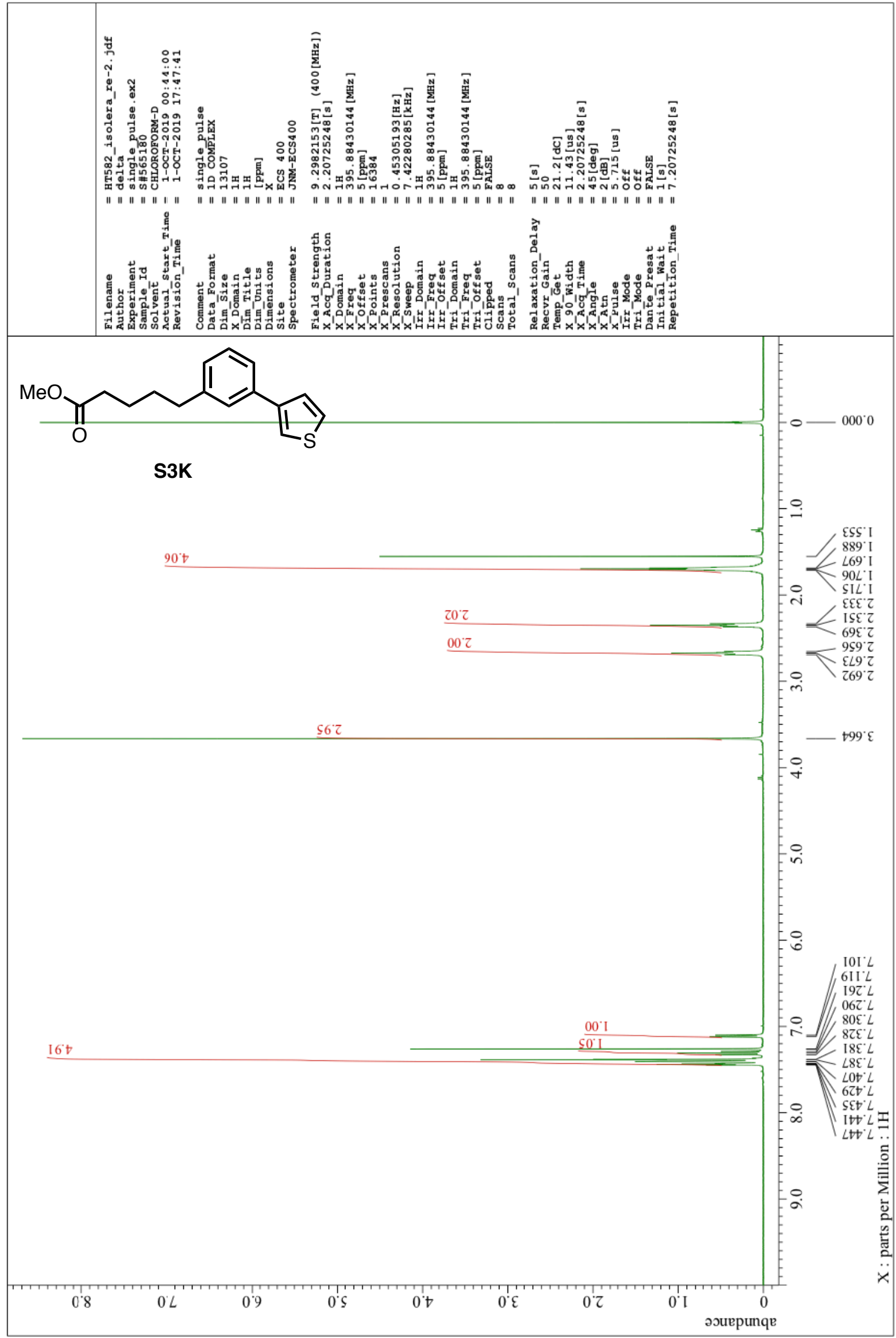


${ }^{13} \mathrm{C}$ NMR of $\mathbf{S 3 K}\left(101 \mathrm{MHz}, \mathrm{CDCl}_{3}\right)$

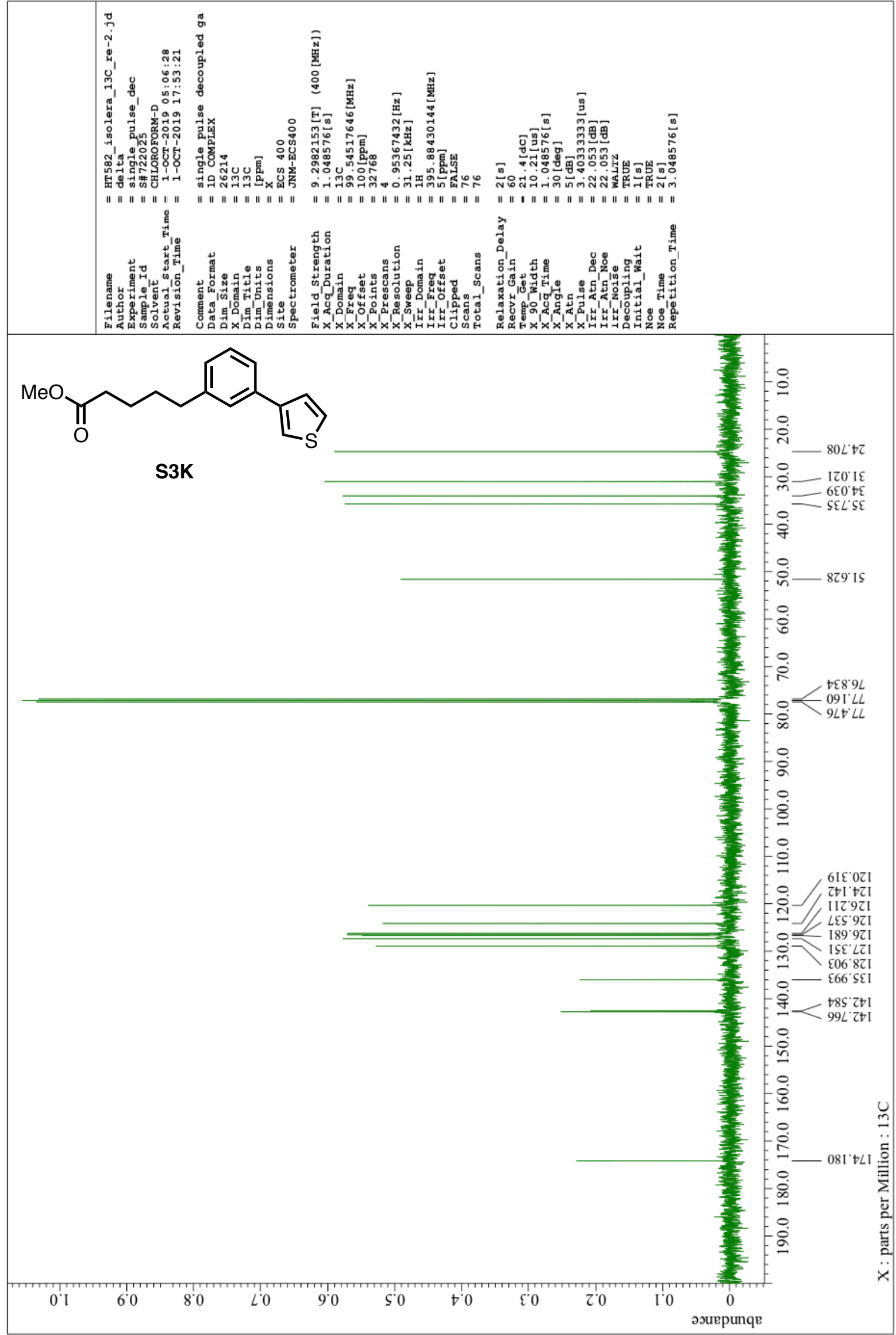


${ }^{1} \mathrm{H}$ NMR of $1 \mathbf{B}\left(400 \mathrm{MHz}, \mathrm{CDCl}_{3}\right)$

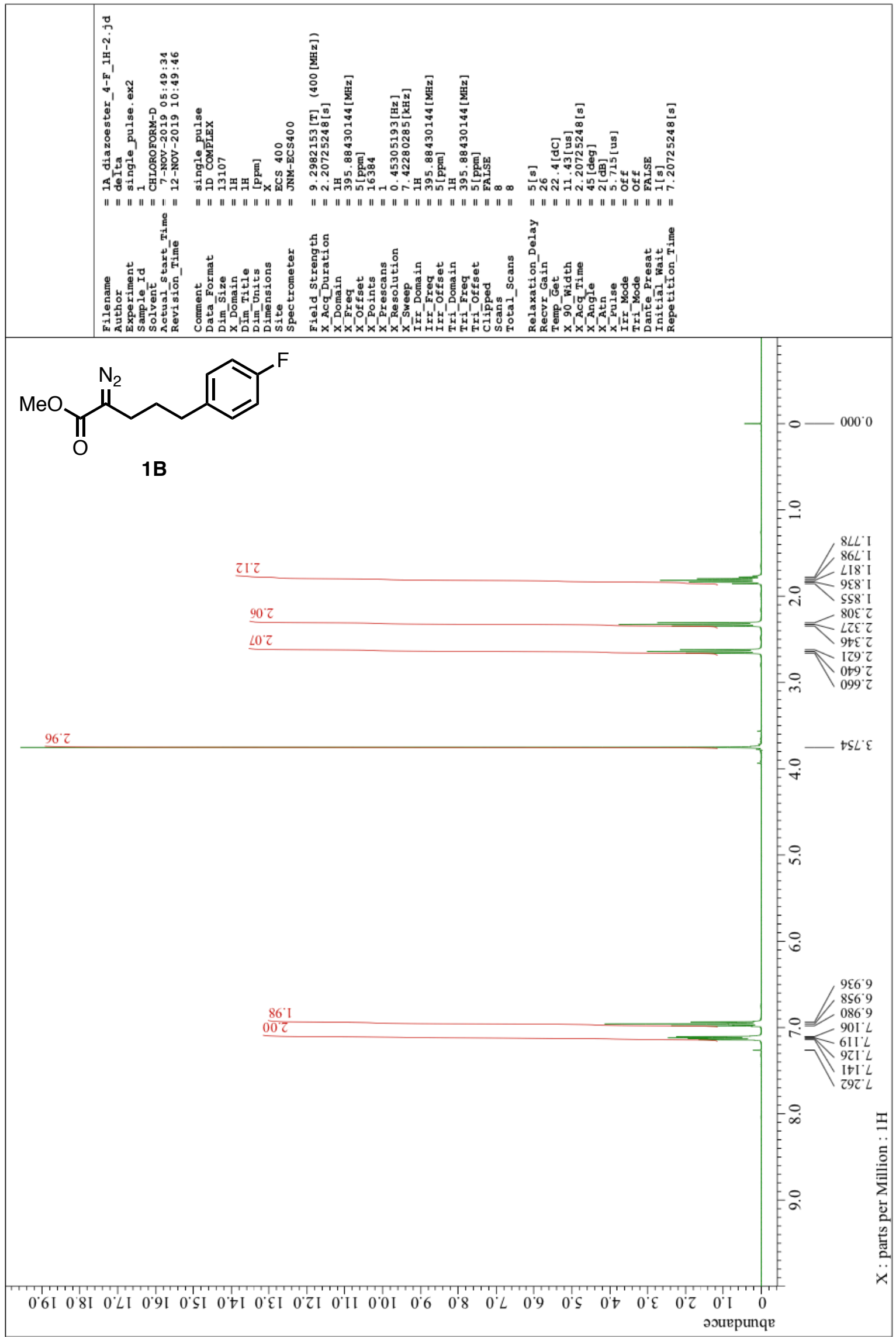


${ }^{13} \mathrm{C}$ NMR of $1 \mathbf{B}\left(101 \mathrm{MHz}, \mathrm{CDCl}_{3}\right)$

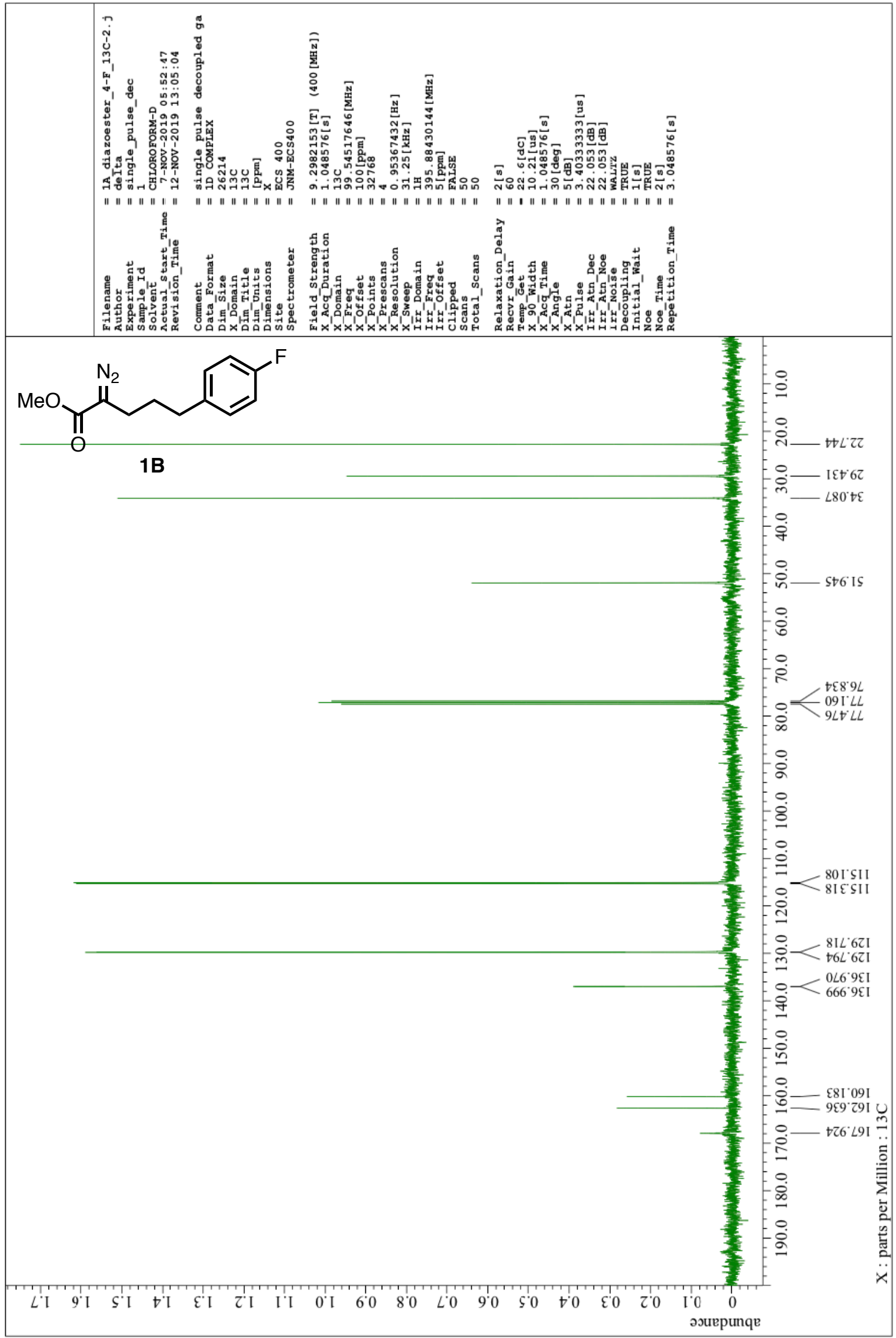


${ }^{1} \mathrm{H}$ NMR of $1 \mathrm{C}\left(400 \mathrm{MHz}, \mathrm{CDCl}_{3}\right)$

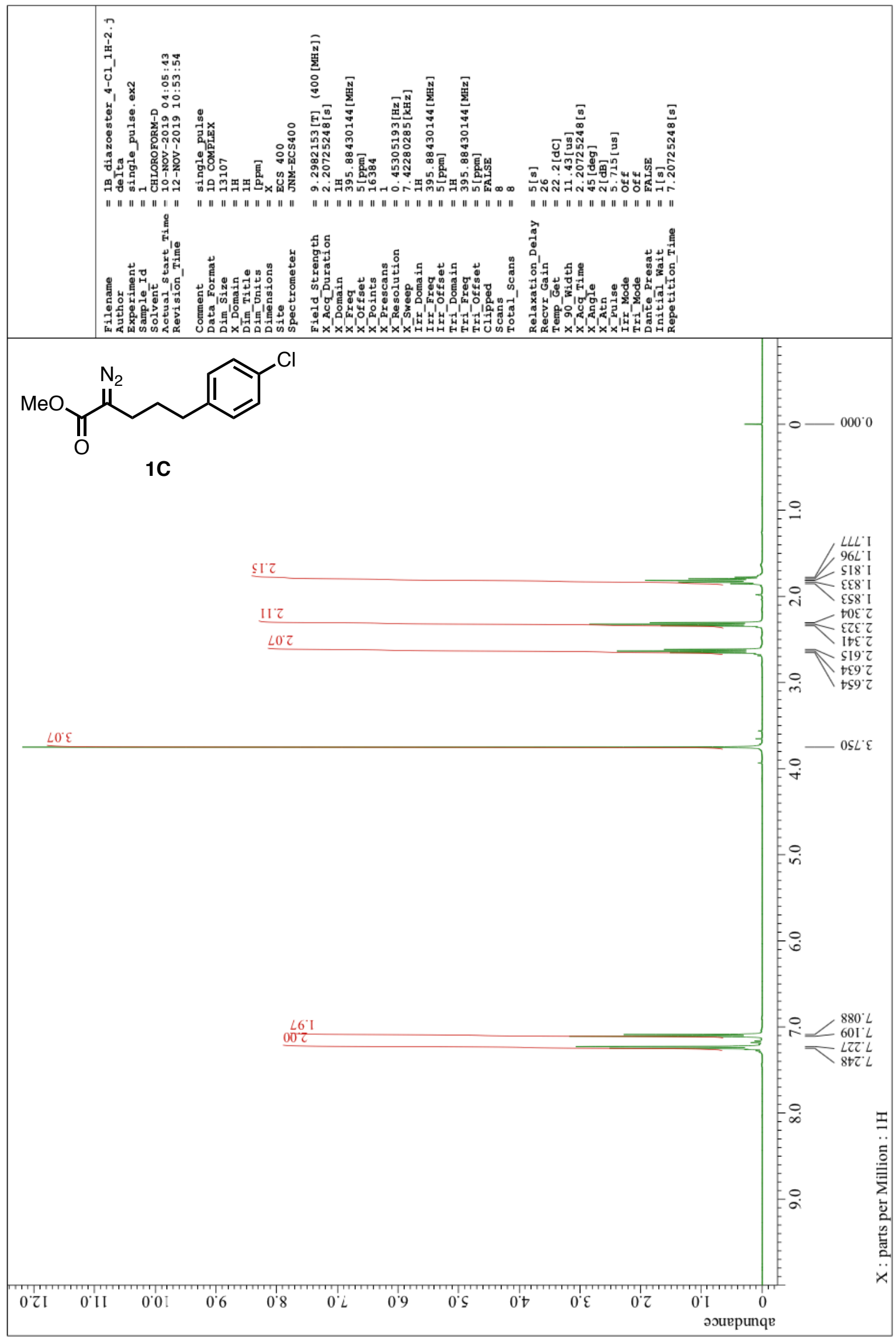


${ }^{13} \mathrm{C}$ NMR of $1 \mathrm{C}\left(101 \mathrm{MHz}, \mathrm{CDCl}_{3}\right)$

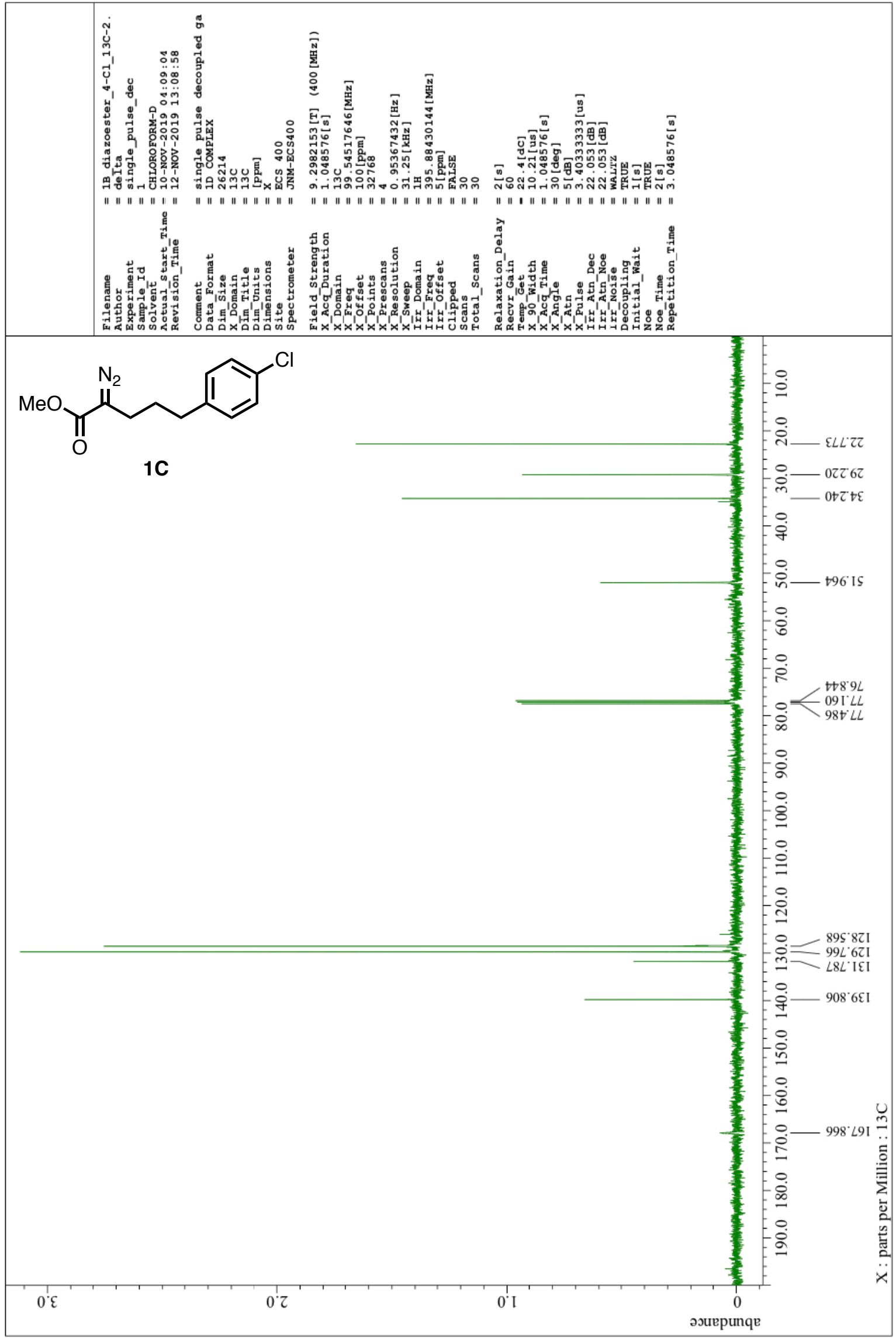


${ }^{1} \mathrm{H}$ NMR of $1 D\left(400 \mathrm{MHz}, \mathrm{CDCl}_{3}\right)$

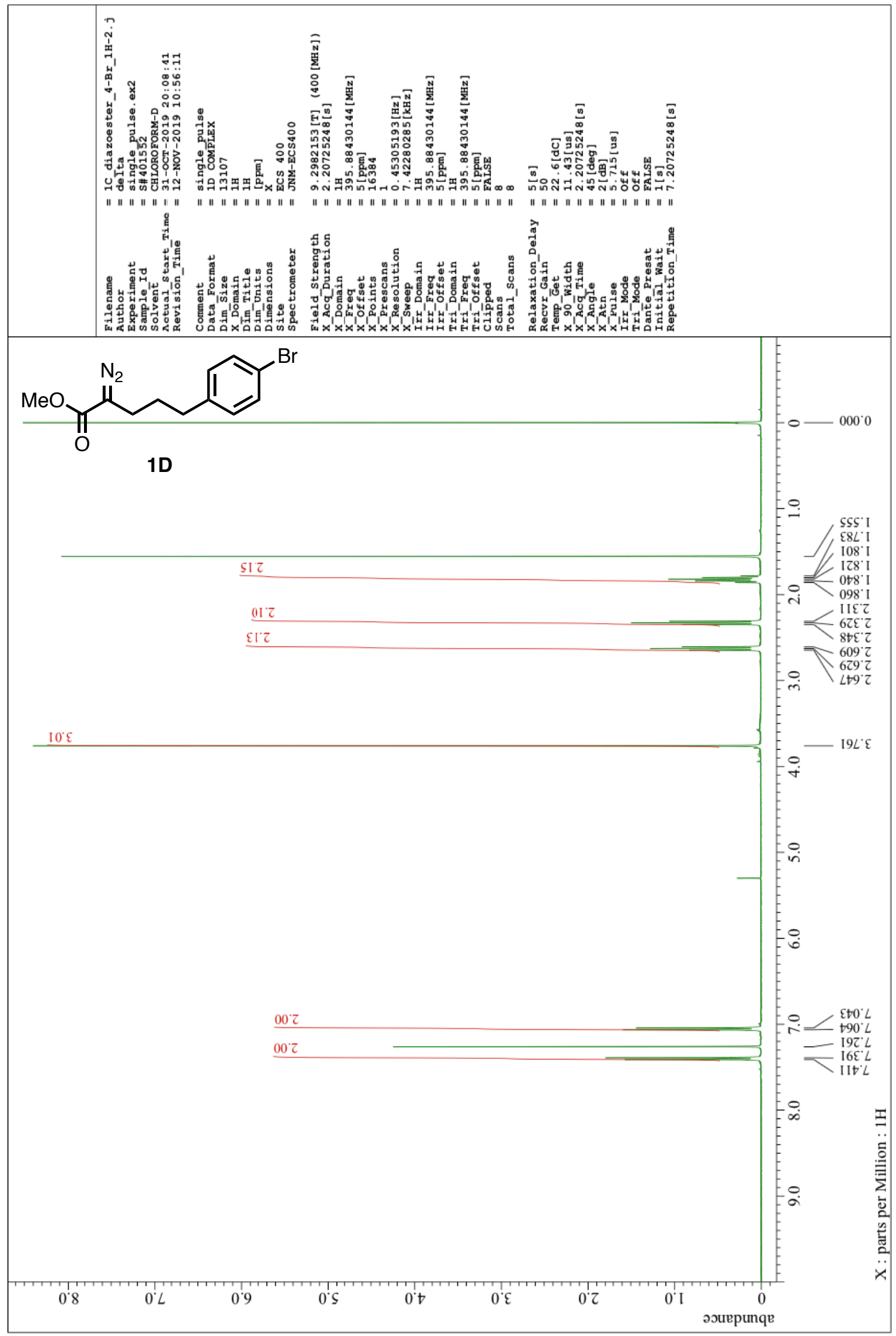


${ }^{13} \mathrm{C}$ NMR of $1 D\left(101 \mathrm{MHz}, \mathrm{CDCl}_{3}\right)$

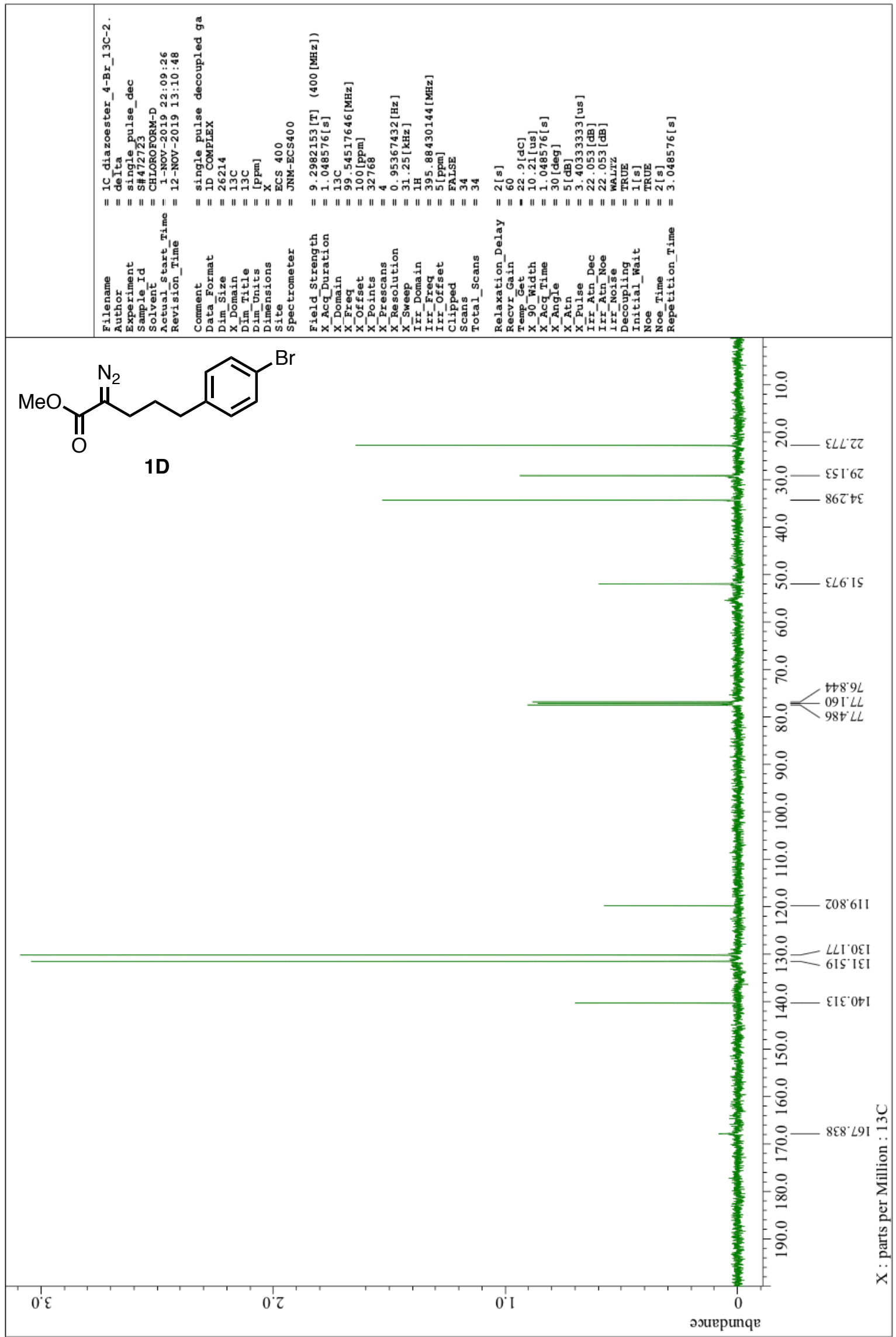


${ }^{1} \mathrm{H}$ NMR of $1 \mathbf{E}\left(400 \mathrm{MHz}, \mathrm{CDCl}_{3}\right)$

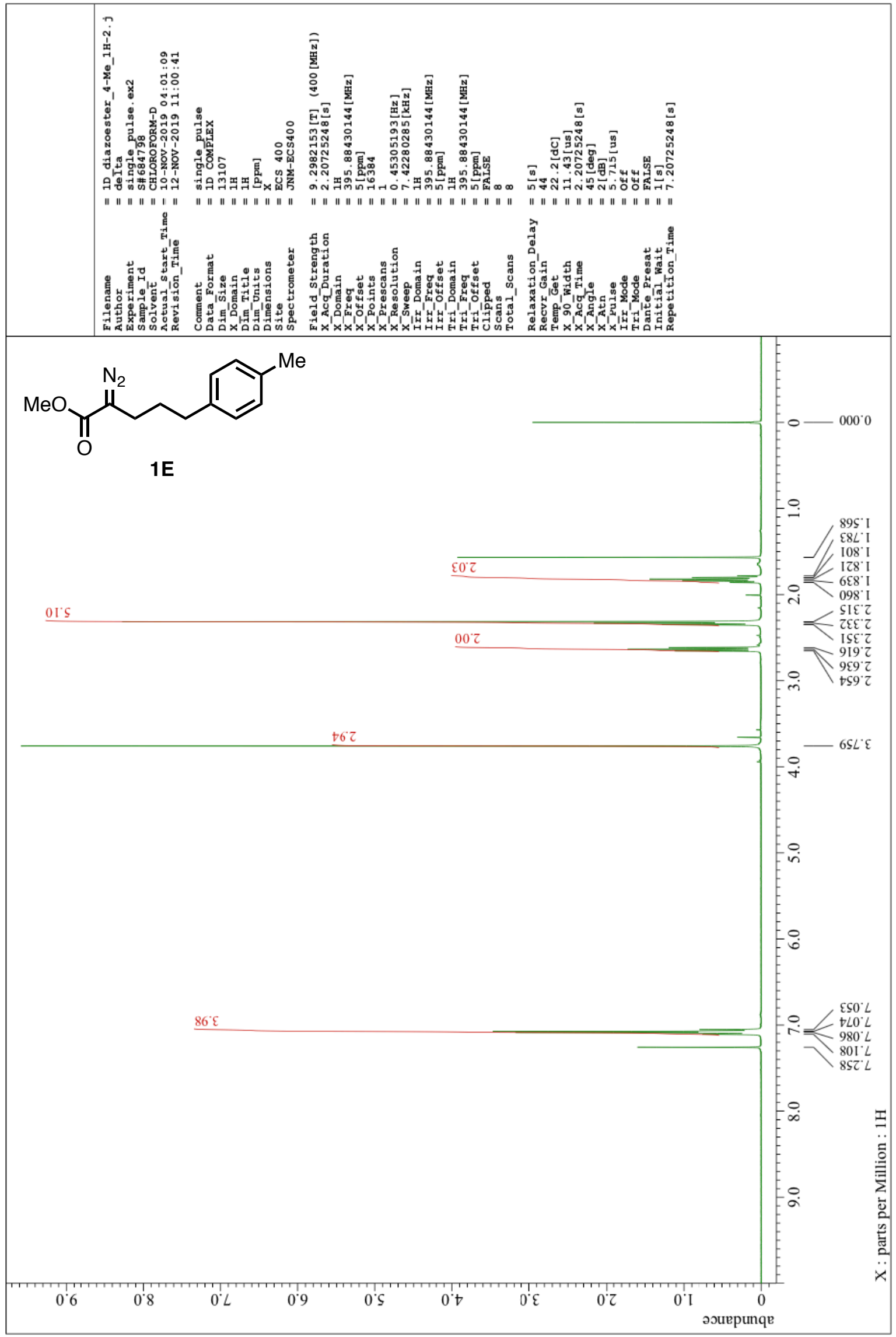


${ }^{13} \mathrm{C}$ NMR of 1E $\left(101 \mathrm{MHz}, \mathrm{CDCl}_{3}\right)$

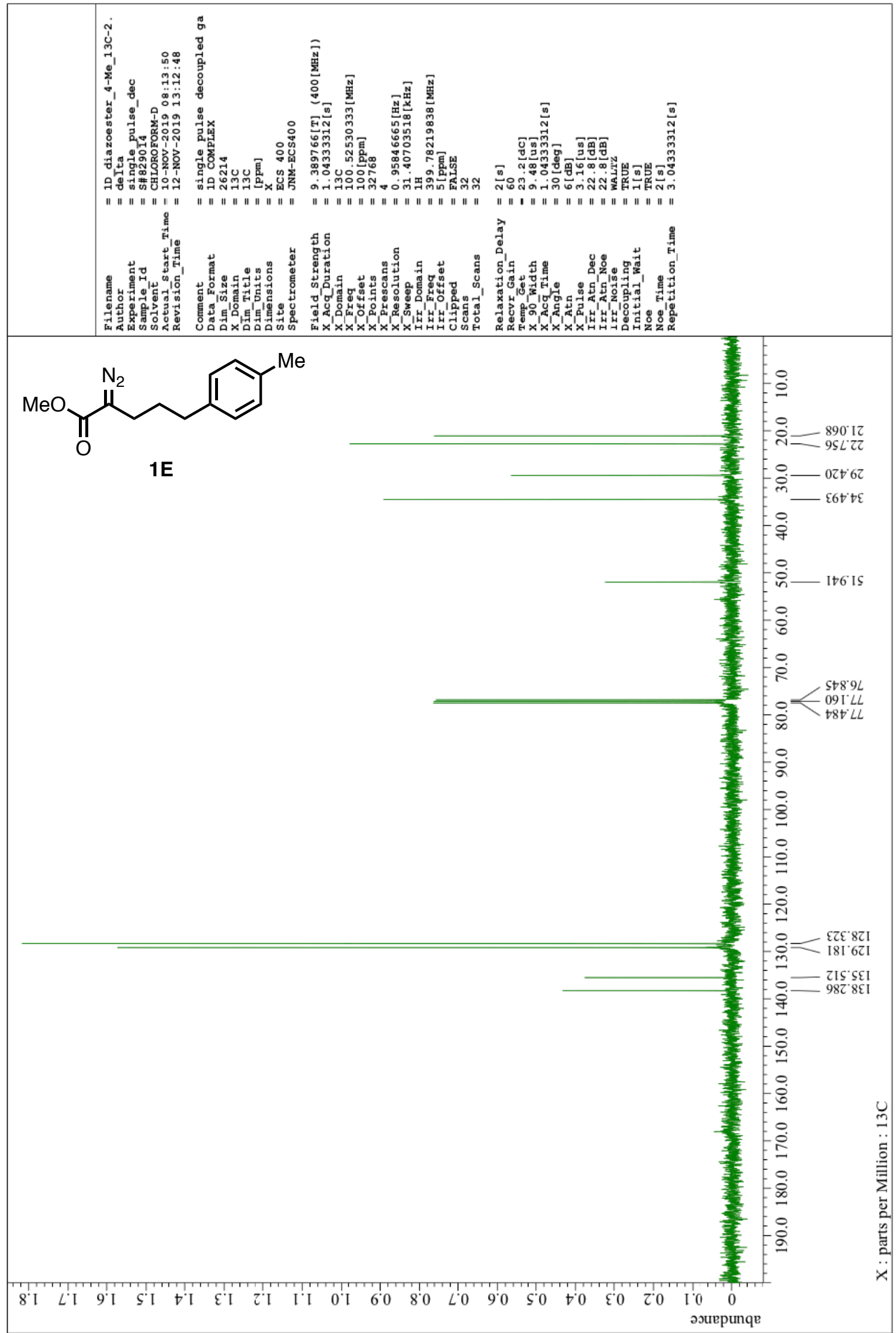


${ }^{1} \mathrm{H}$ NMR of $\mathbf{1 F}\left(400 \mathrm{MHz}, \mathrm{CDCl}_{3}\right)$

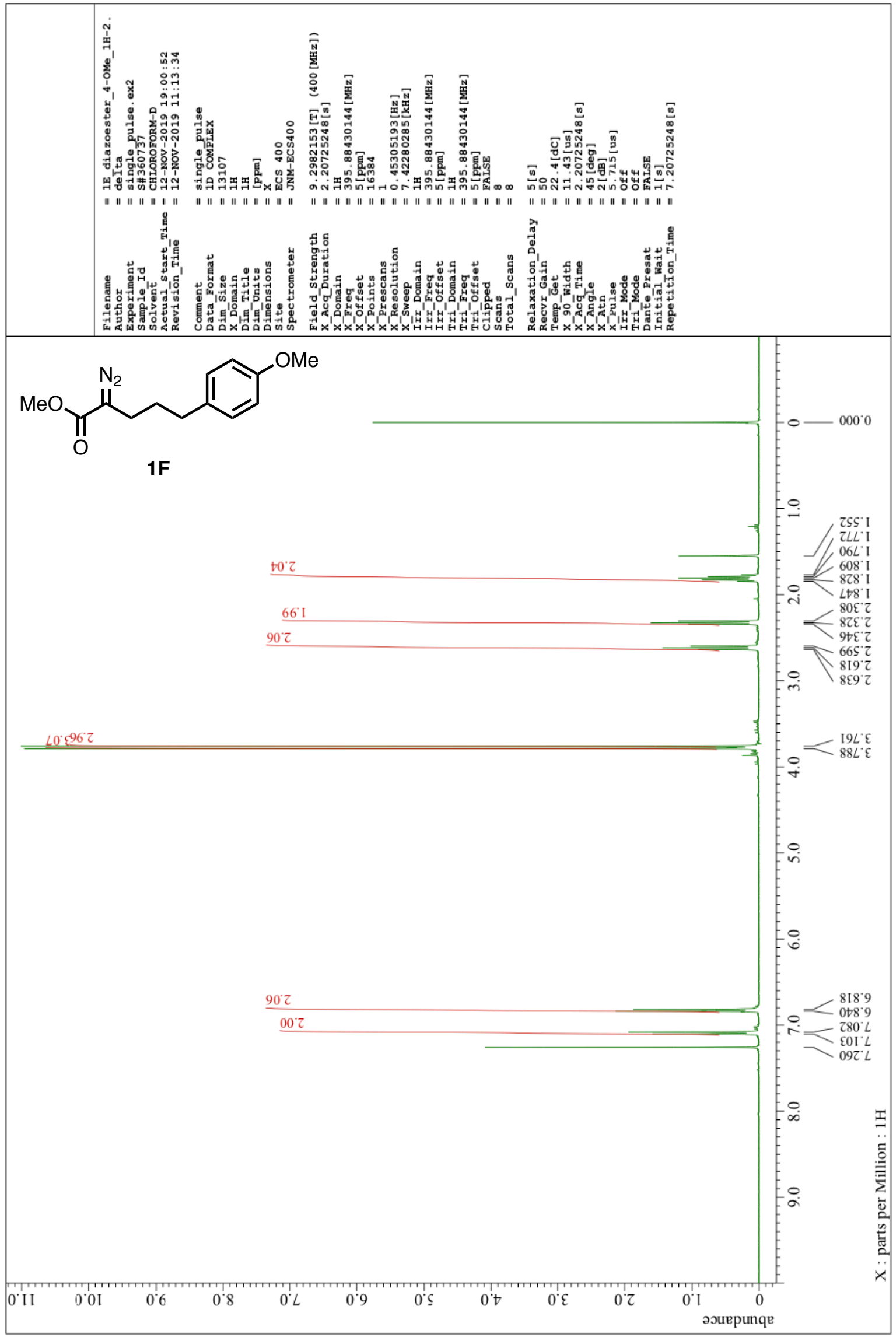


${ }^{13} \mathrm{C}$ NMR of $\mathbf{1 F}\left(101 \mathrm{MHz}, \mathrm{CDCl}_{3}\right)$

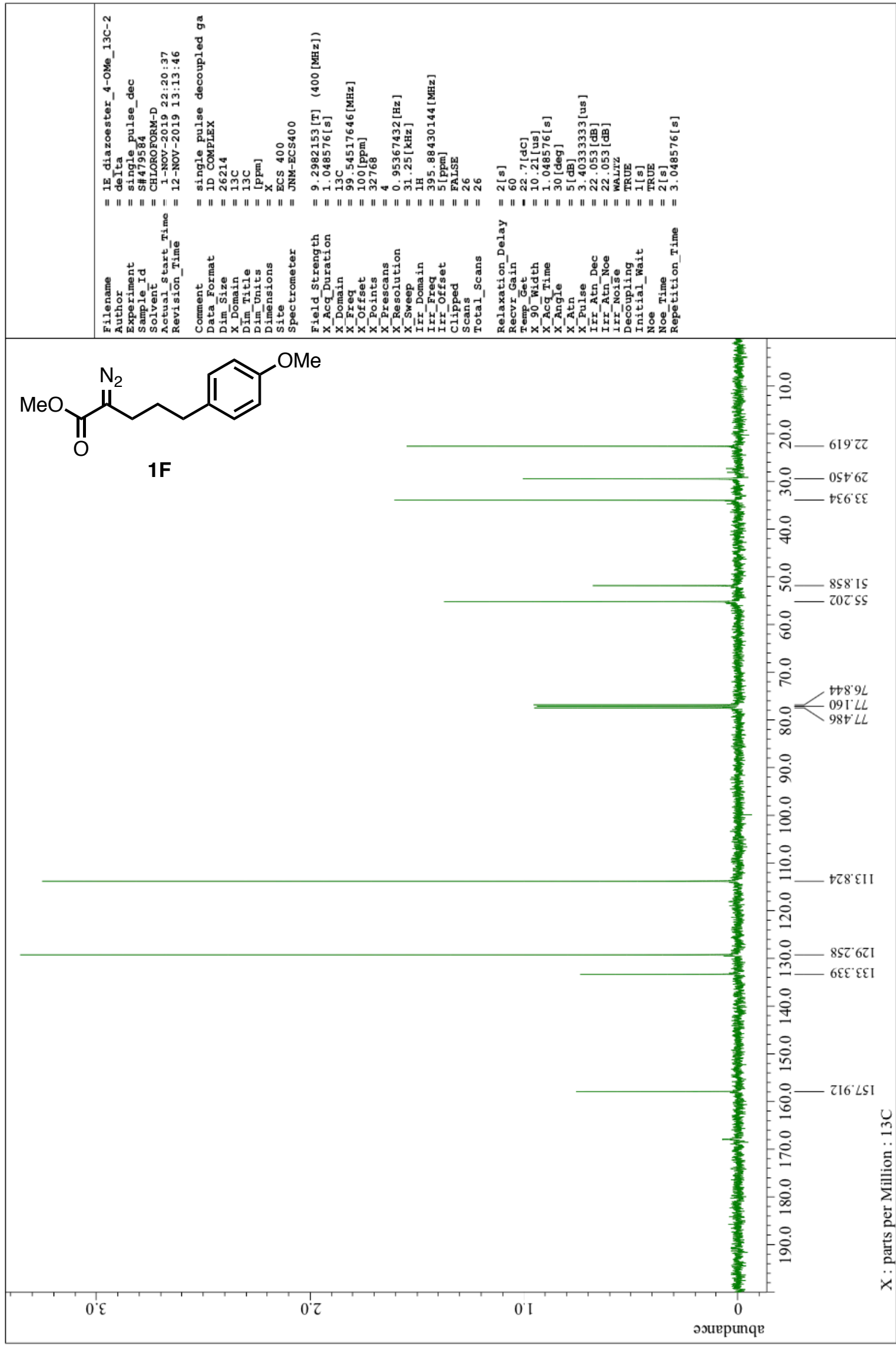


${ }^{1} \mathrm{H}$ NMR of $4\left(400 \mathrm{MHz}, \mathrm{CDCl}_{3}\right)$

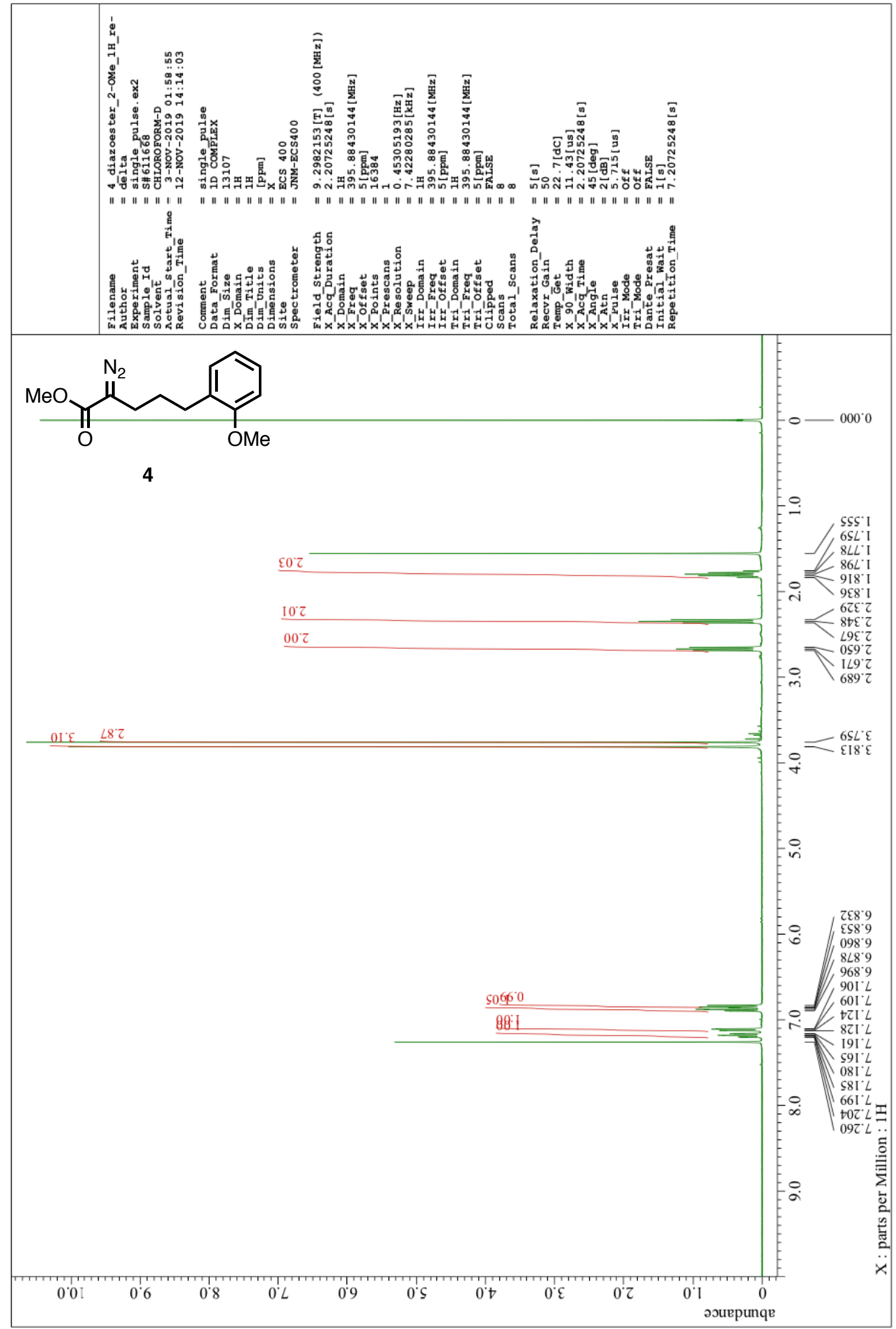


${ }^{13} \mathrm{C}$ NMR of $4\left(101 \mathrm{MHz}, \mathrm{CDCl}_{3}\right)$

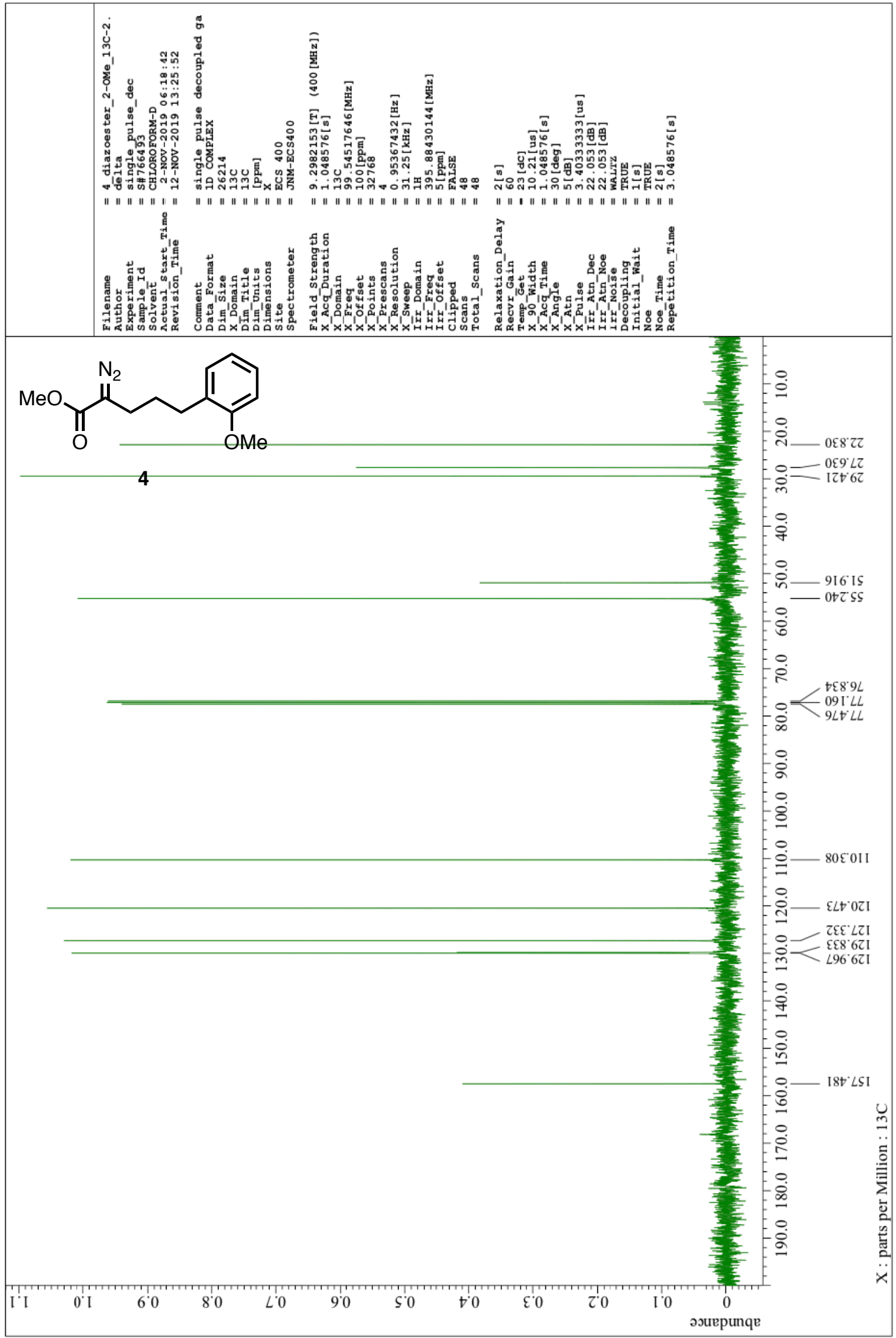


${ }^{1} \mathrm{H}$ NMR of 7A $\left(400 \mathrm{MHz}, \mathrm{CDCl}_{3}\right)$

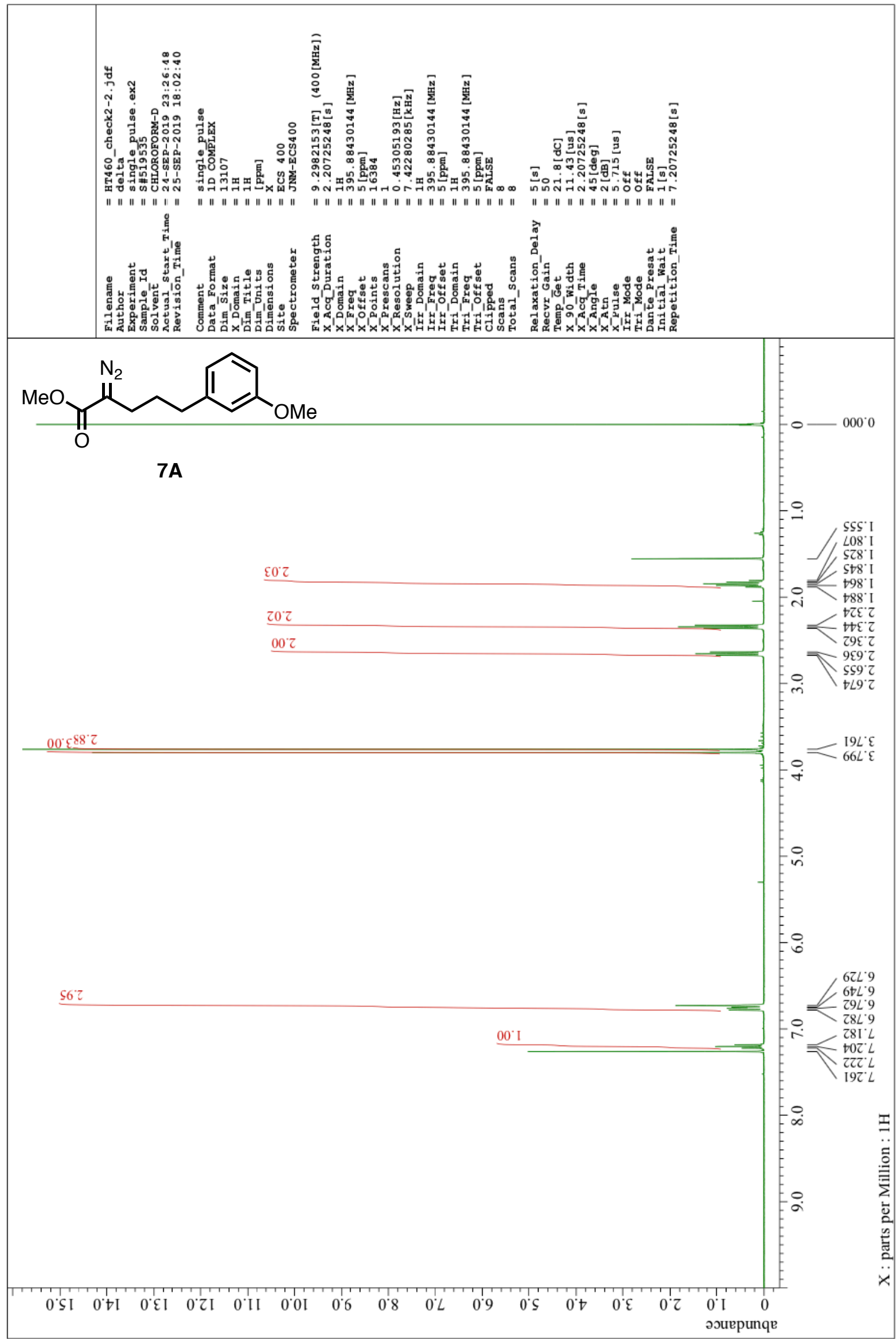


${ }^{13} \mathrm{C}$ NMR of $7 \mathbf{A}\left(101 \mathrm{MHz}, \mathrm{CDCl}_{3}\right)$

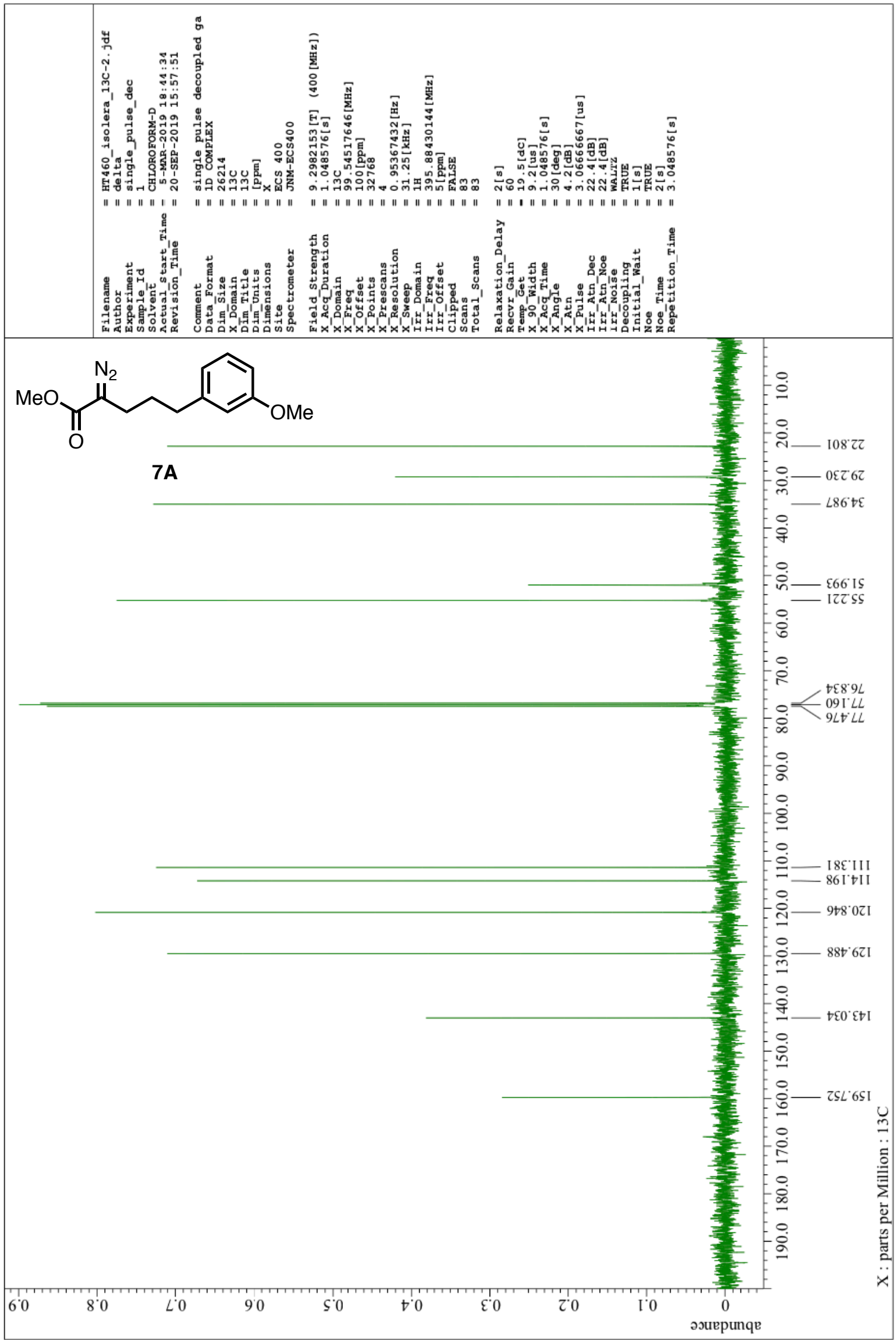


${ }^{1} \mathrm{H}$ NMR of 7B $\left(400 \mathrm{MHz}, \mathrm{CDCl}_{3}\right)$

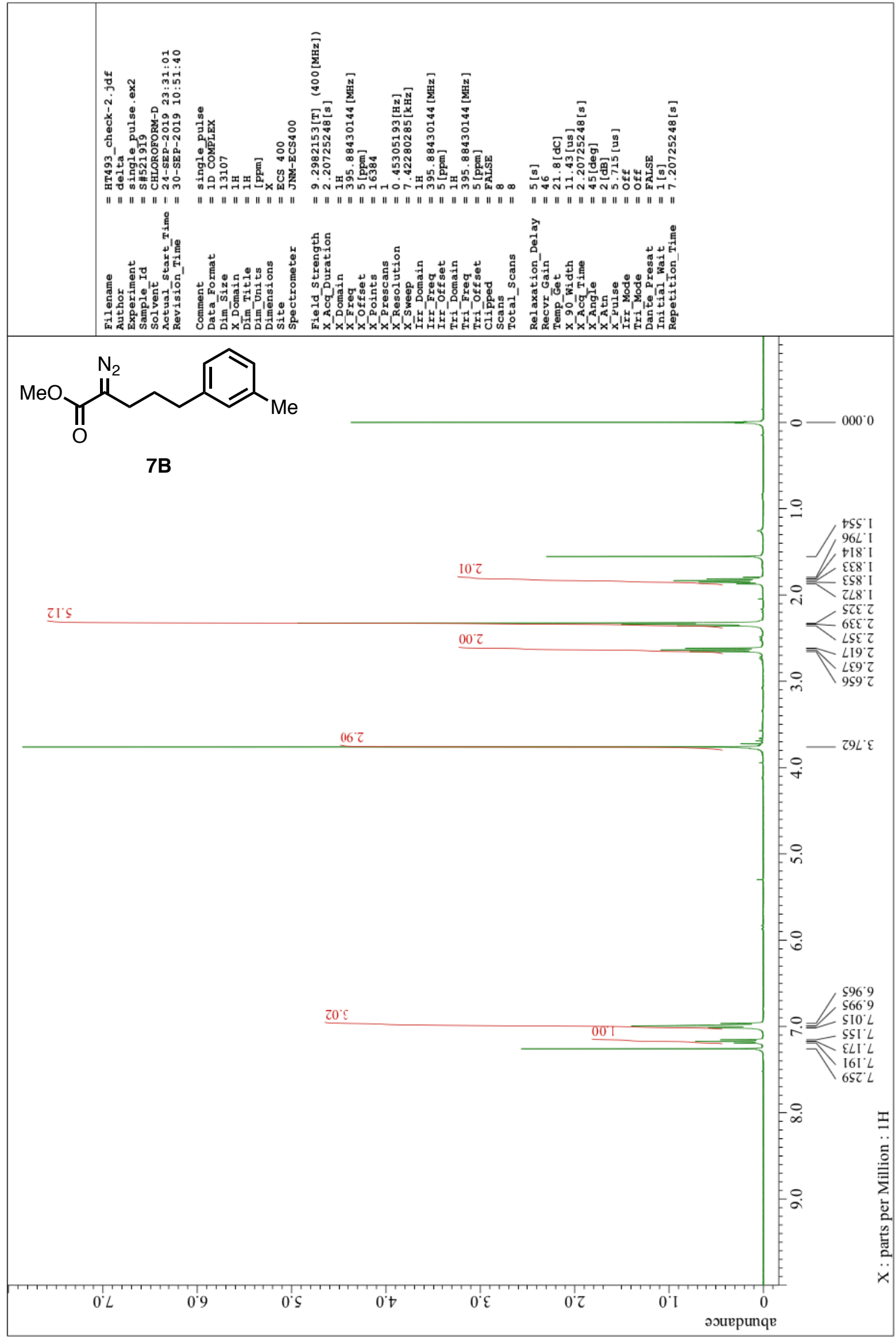


${ }^{13} \mathrm{C}$ NMR of 7B $\left(101 \mathrm{MHz}, \mathrm{CDCl}_{3}\right)$

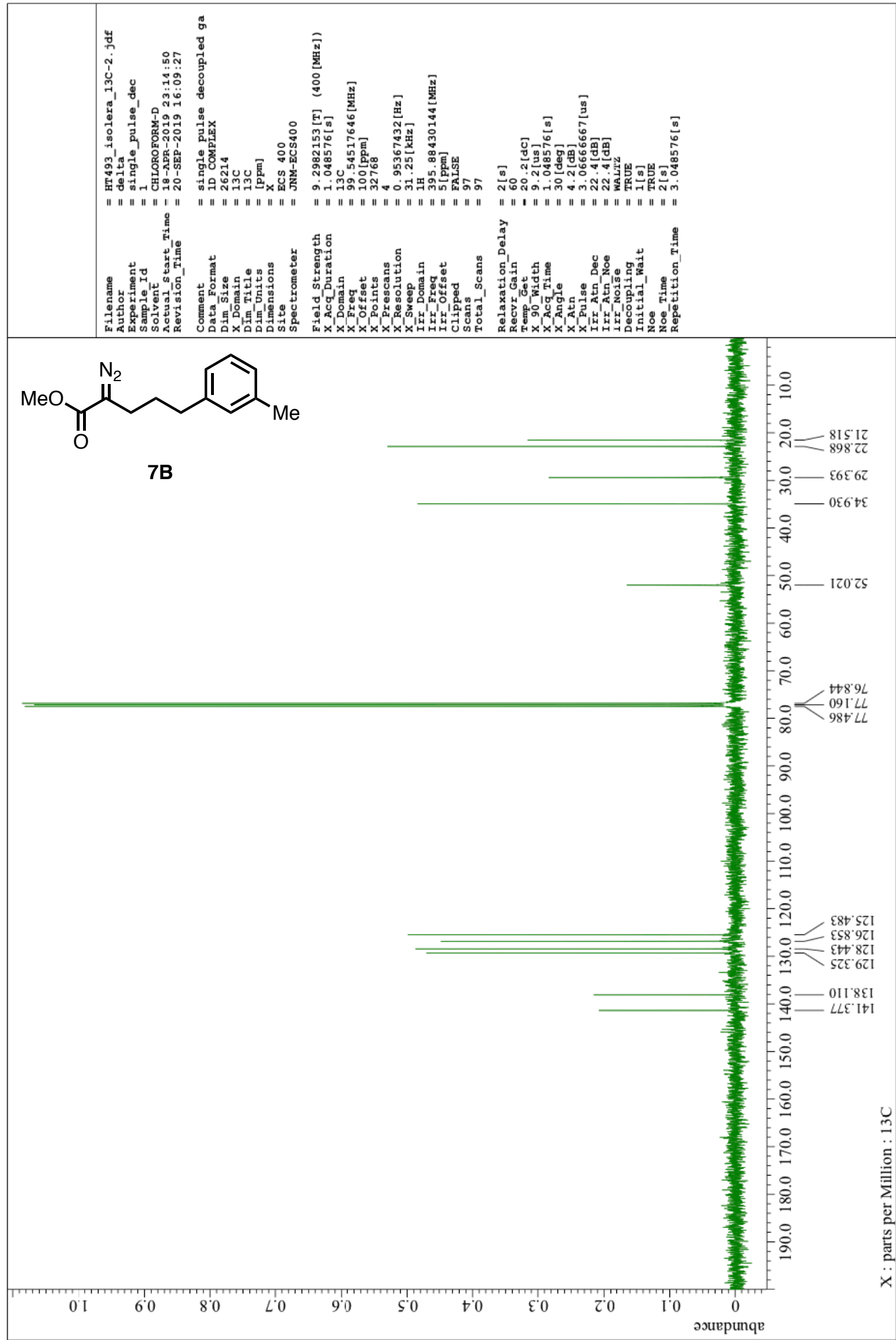


${ }^{1} \mathrm{H}$ NMR of $7 \mathrm{C}\left(400 \mathrm{MHz}, \mathrm{CDCl}_{3}\right)$

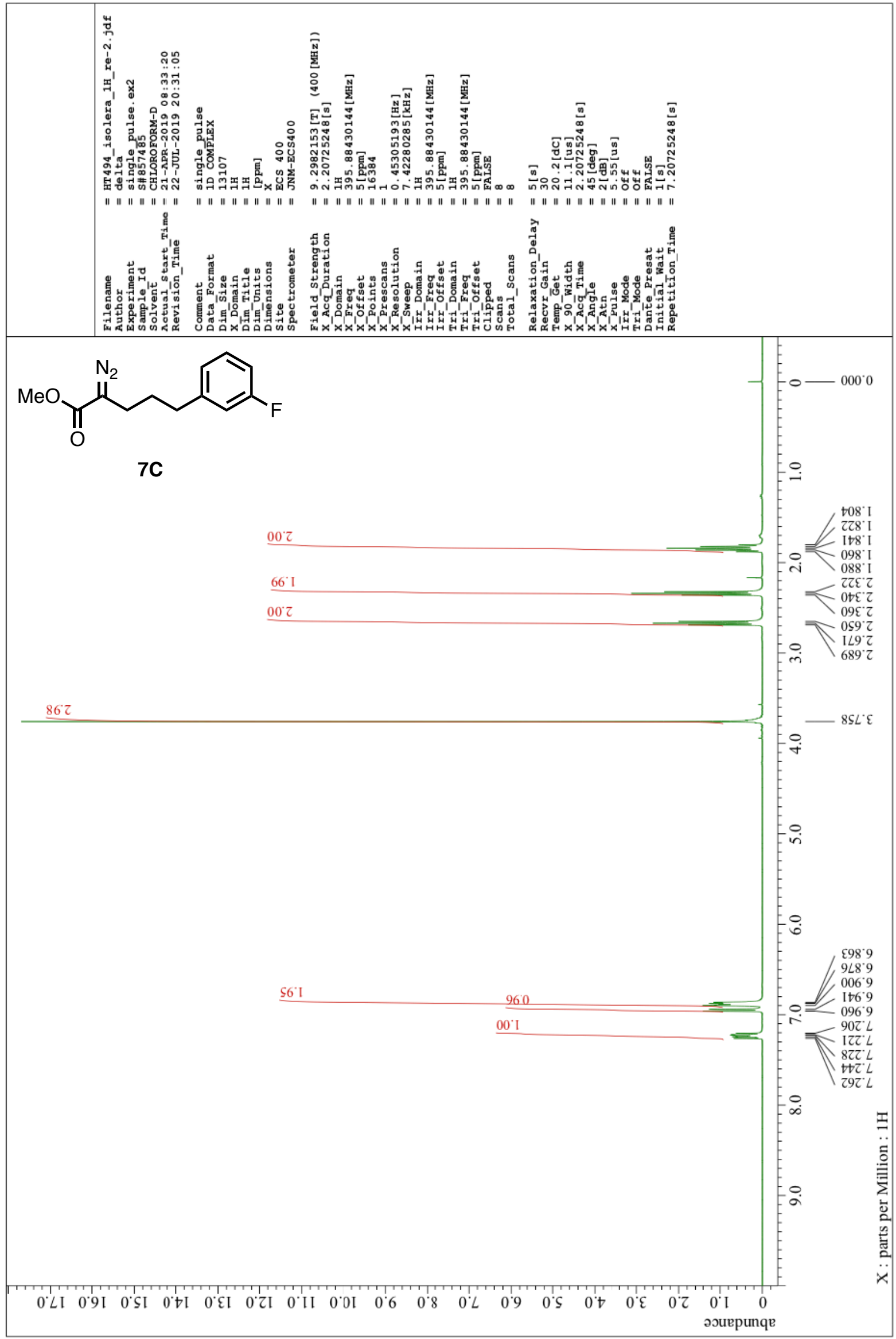


${ }^{13} \mathrm{C}$ NMR of $7 \mathrm{C}\left(101 \mathrm{MHz}, \mathrm{CDCl}_{3}\right)$

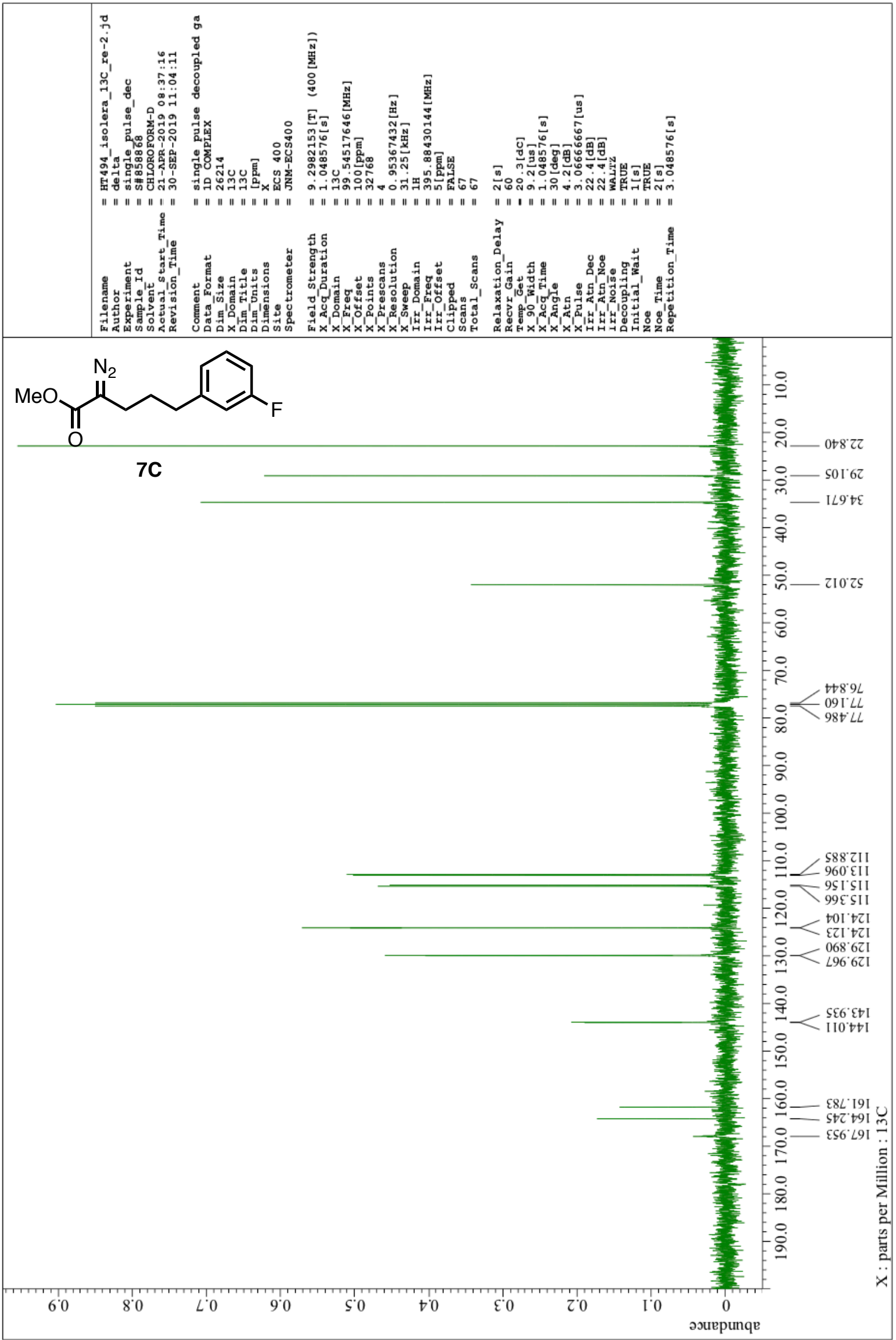


${ }^{1} \mathrm{H}$ NMR of 7D $\left(400 \mathrm{MHz}, \mathrm{CDCl}_{3}\right)$

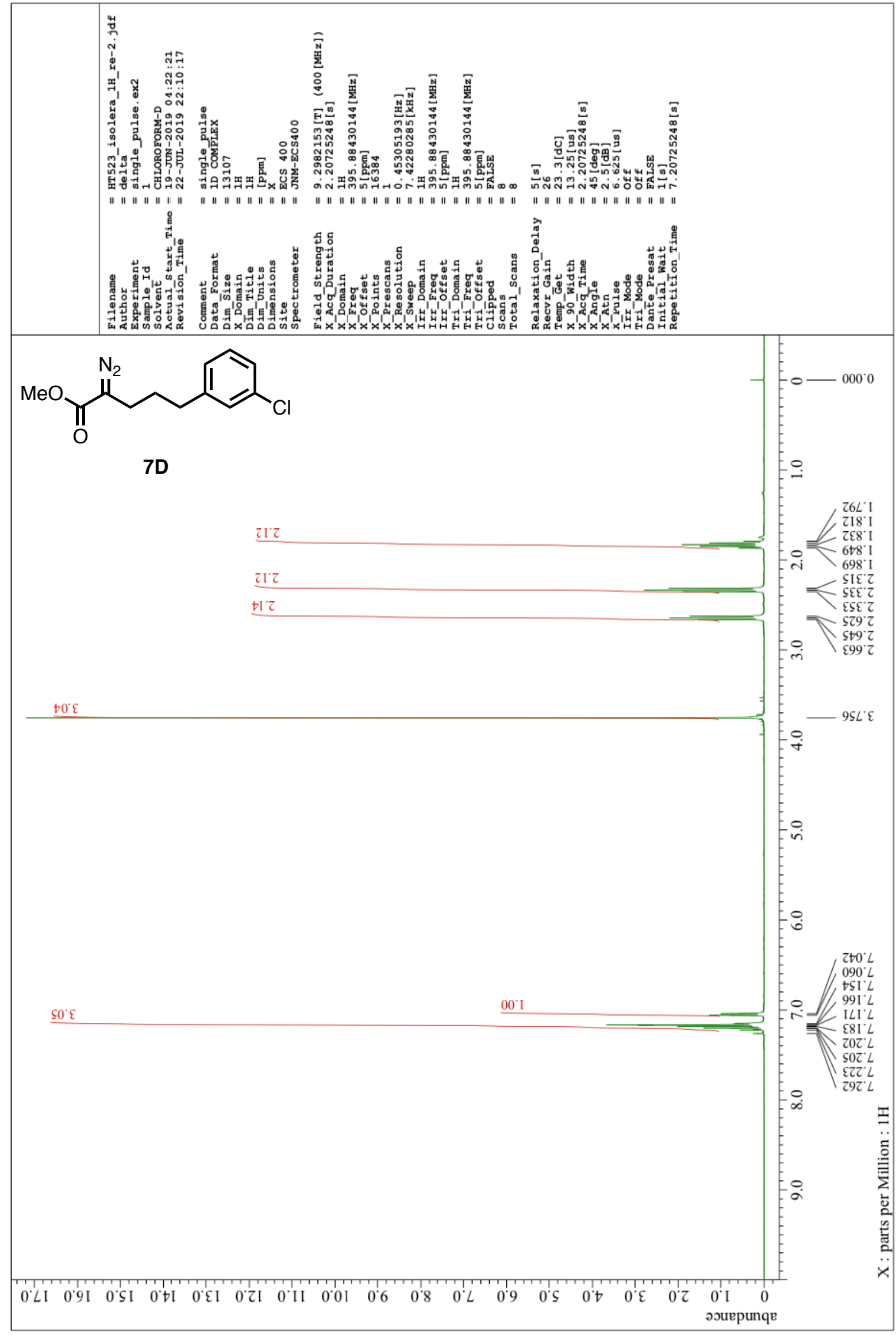


${ }^{13} \mathrm{C}$ NMR of 7D $\left(101 \mathrm{MHz}, \mathrm{CDCl}_{3}\right)$

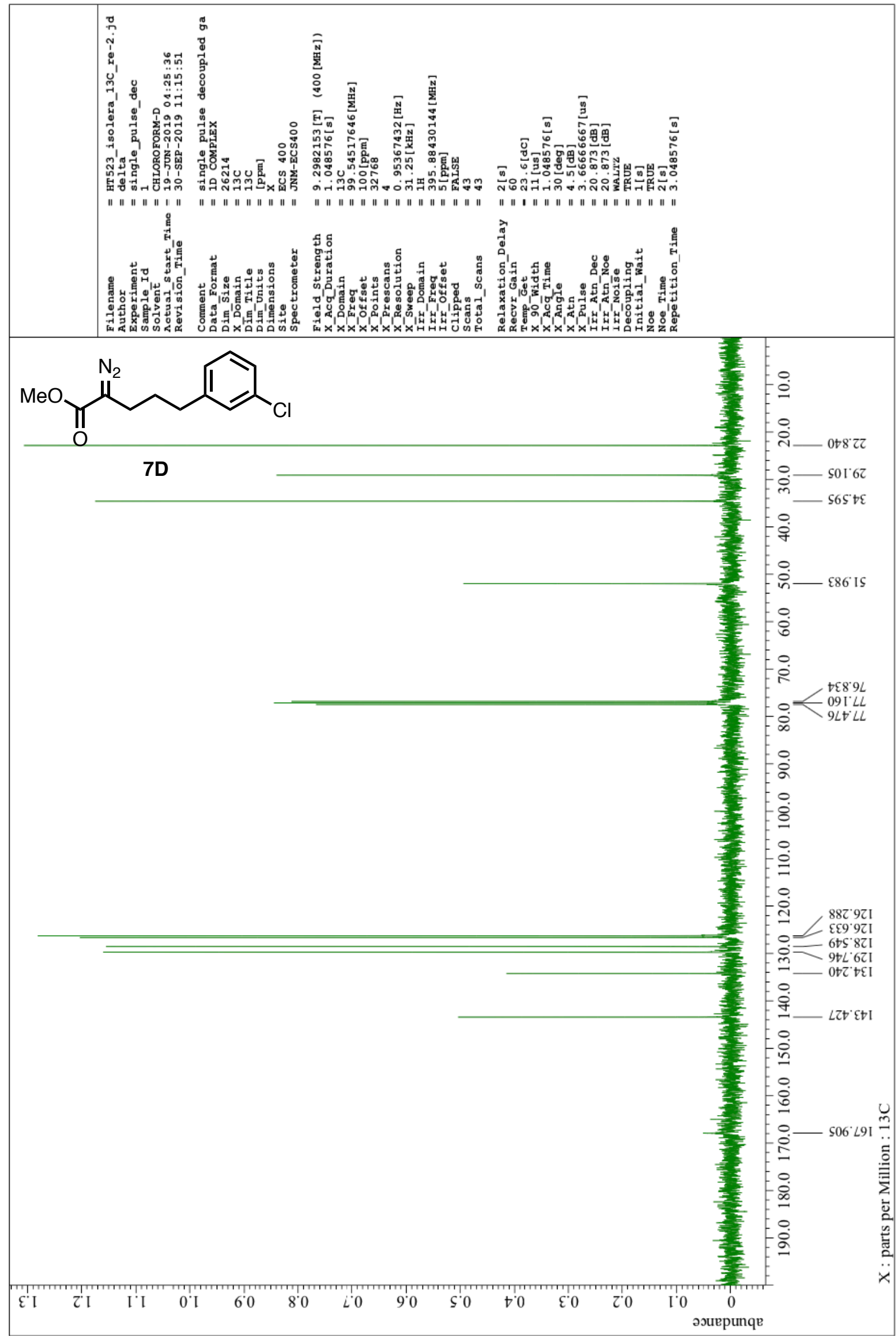


${ }^{1} \mathrm{H}$ NMR of $7 \mathbf{E}\left(400 \mathrm{MHz}, \mathrm{CDCl}_{3}\right)$

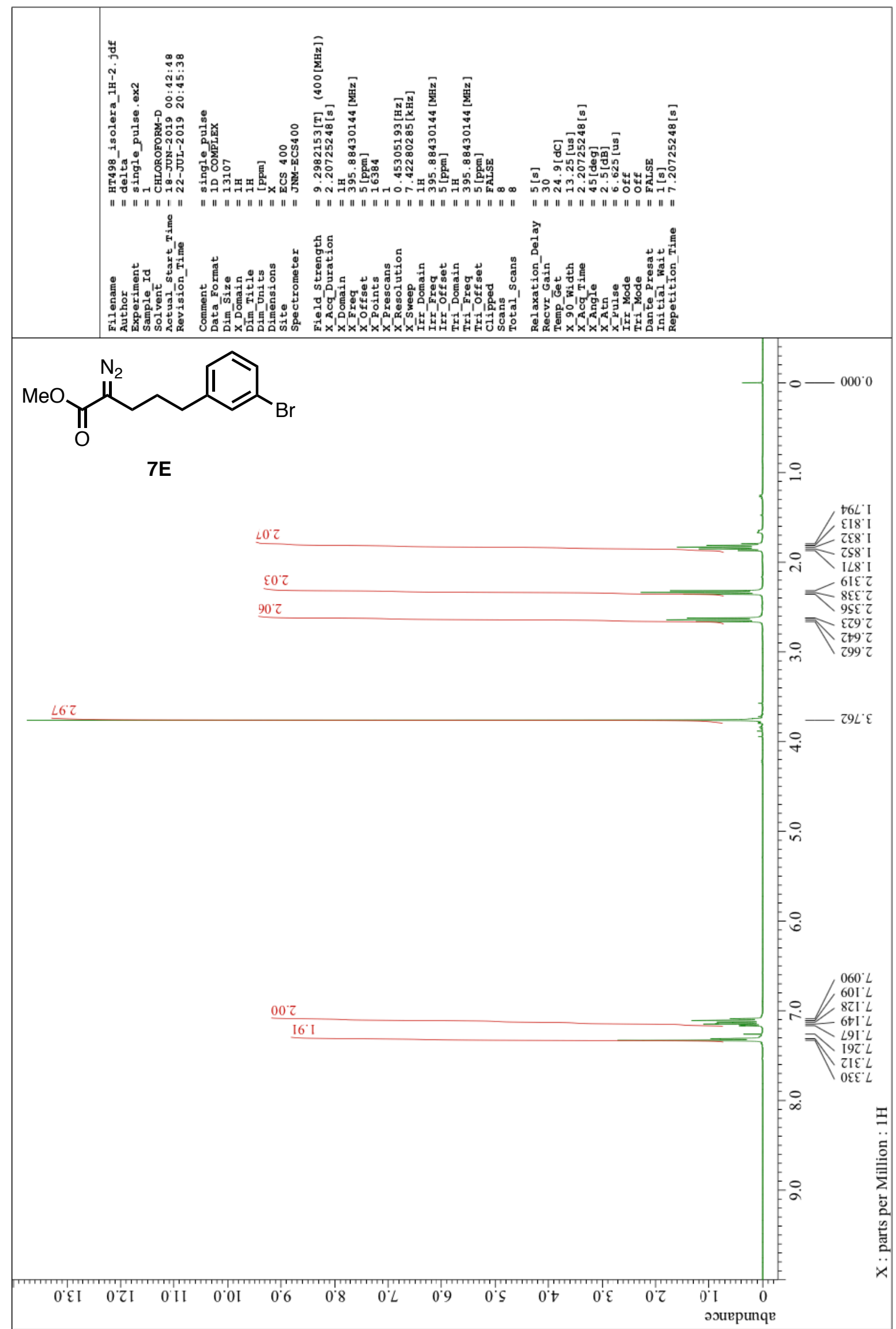


${ }^{13} \mathrm{C}$ NMR of $7 \mathbf{E}\left(101 \mathrm{MHz}, \mathrm{CDCl}_{3}\right)$

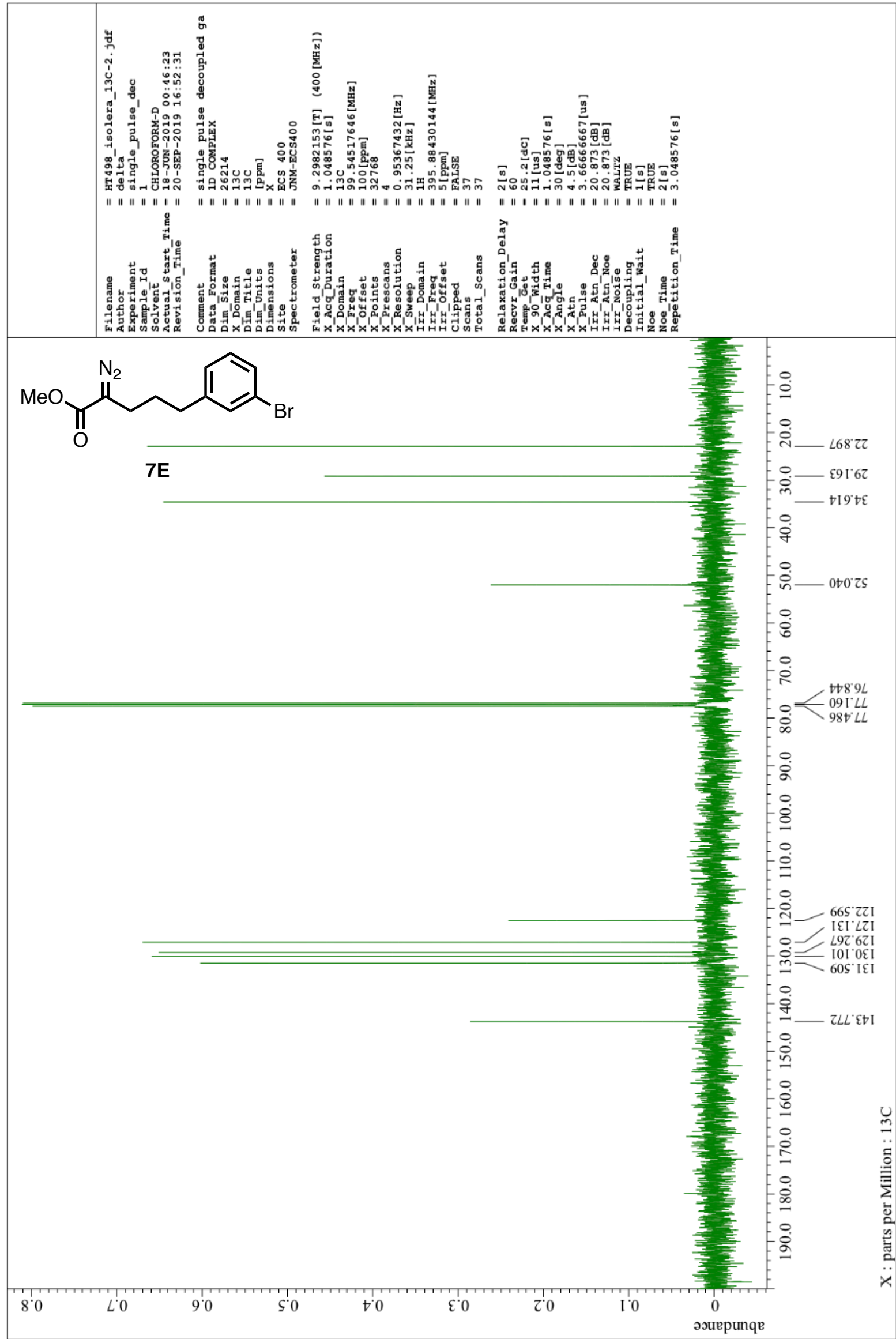


${ }^{1} \mathrm{H}$ NMR of $7 \mathbf{F}\left(400 \mathrm{MHz}, \mathrm{CDCl}_{3}\right)$

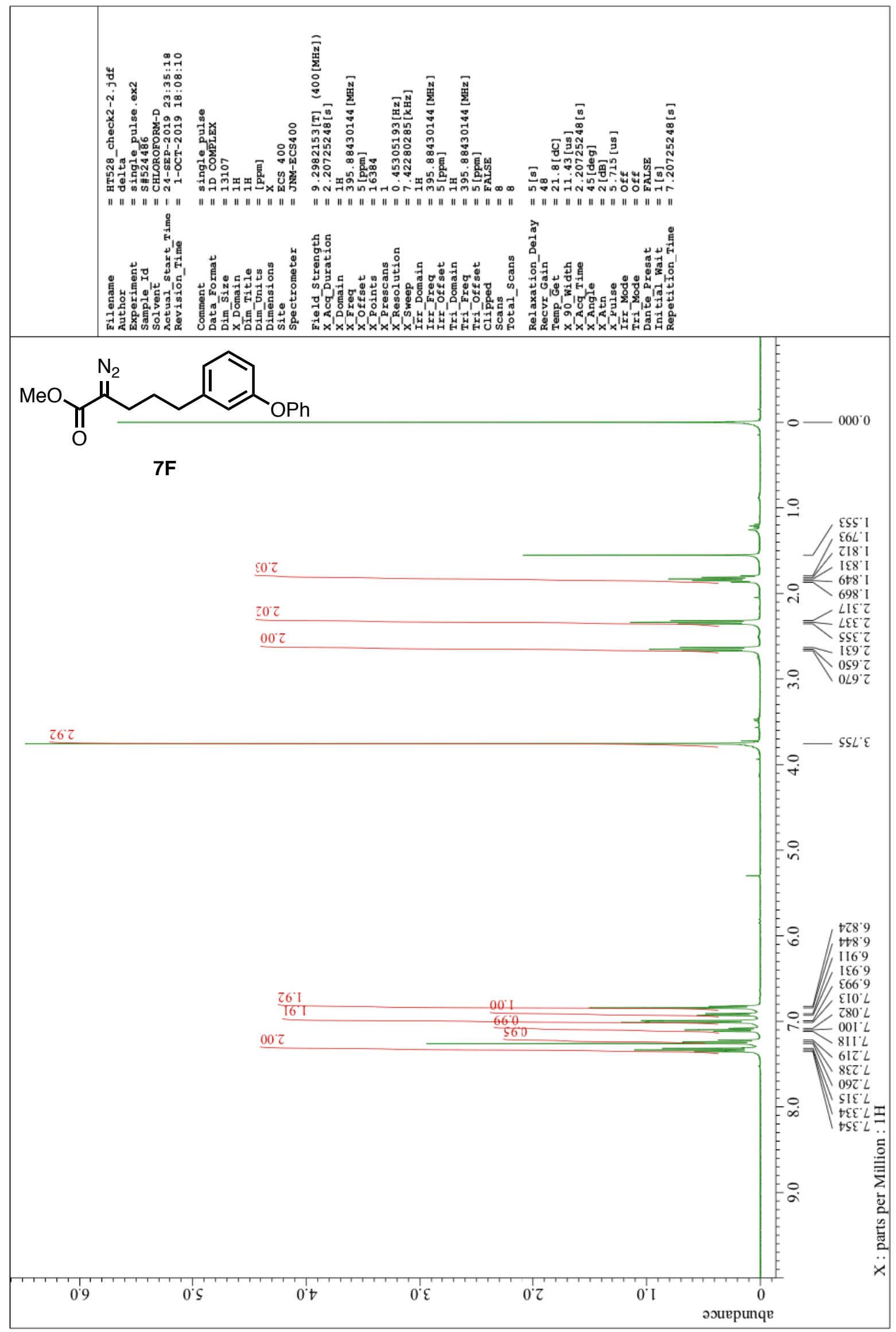


${ }^{13} \mathrm{C}$ NMR of $7 \mathbf{F}\left(101 \mathrm{MHz}, \mathrm{CDCl}_{3}\right)$

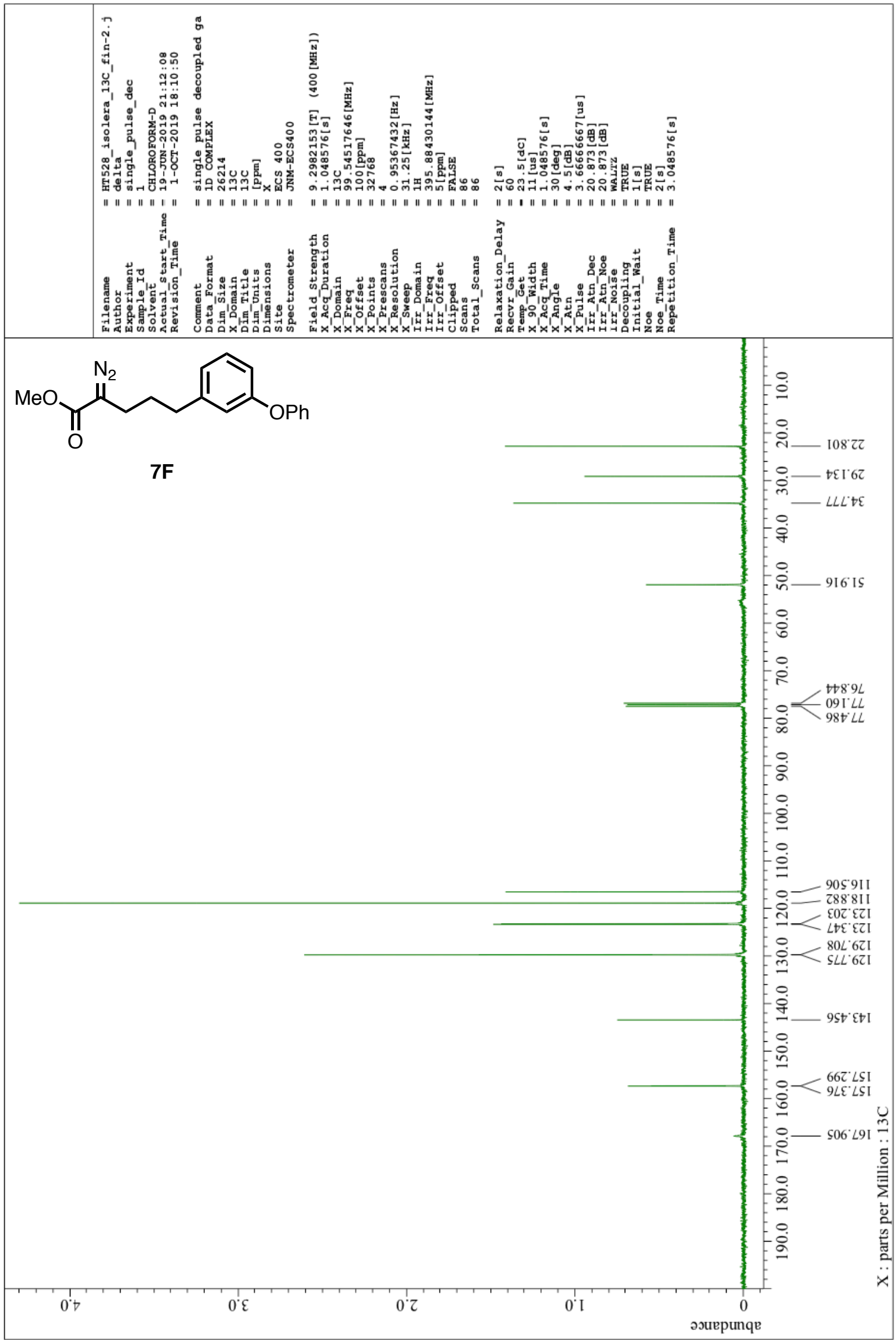


${ }^{1} \mathrm{H}$ NMR of $7 \mathbf{G}\left(400 \mathrm{MHz}, \mathrm{CDCl}_{3}\right)$

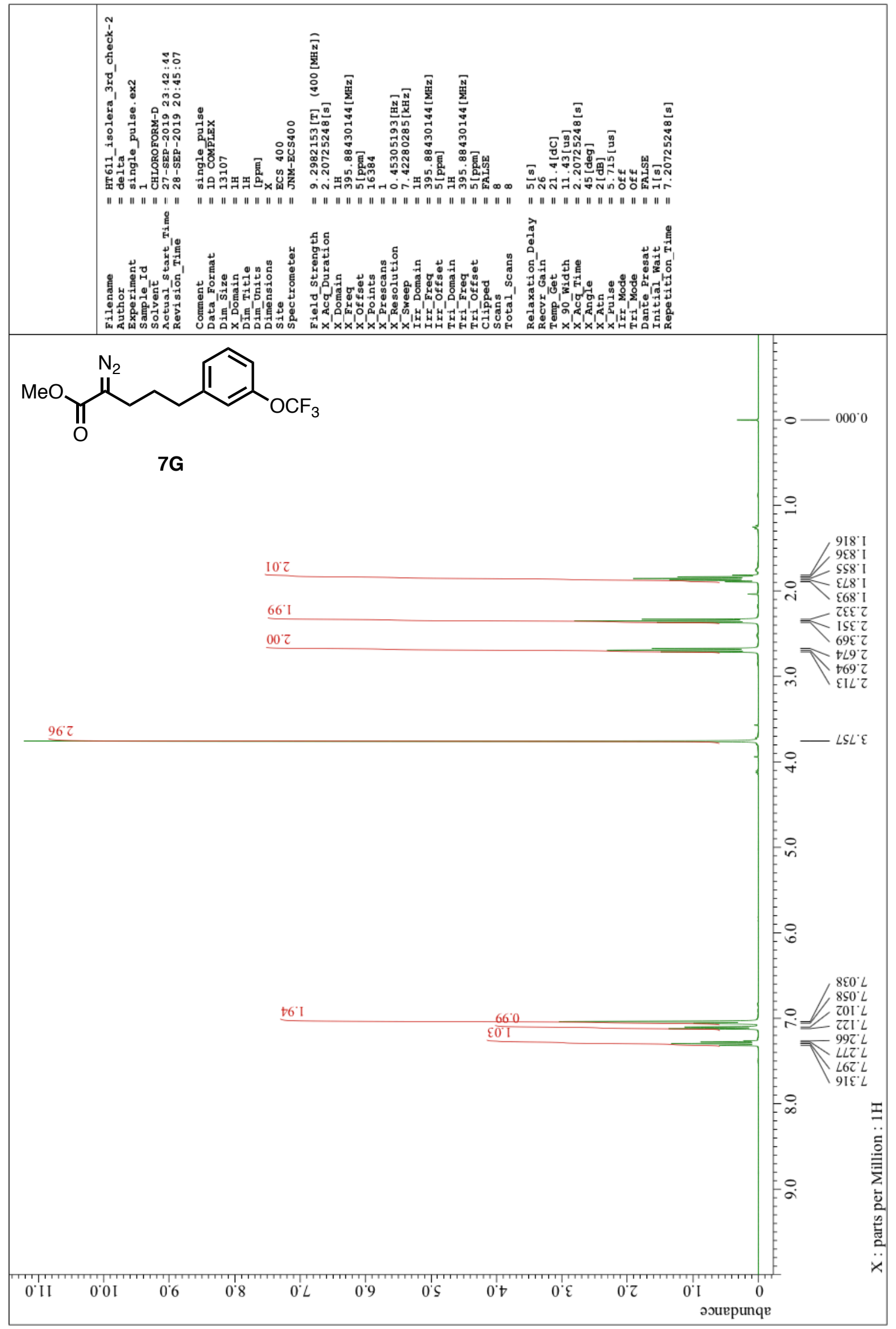


${ }^{13} \mathrm{C}$ NMR of $7 \mathbf{G}\left(101 \mathrm{MHz}, \mathrm{CDCl}_{3}\right)$

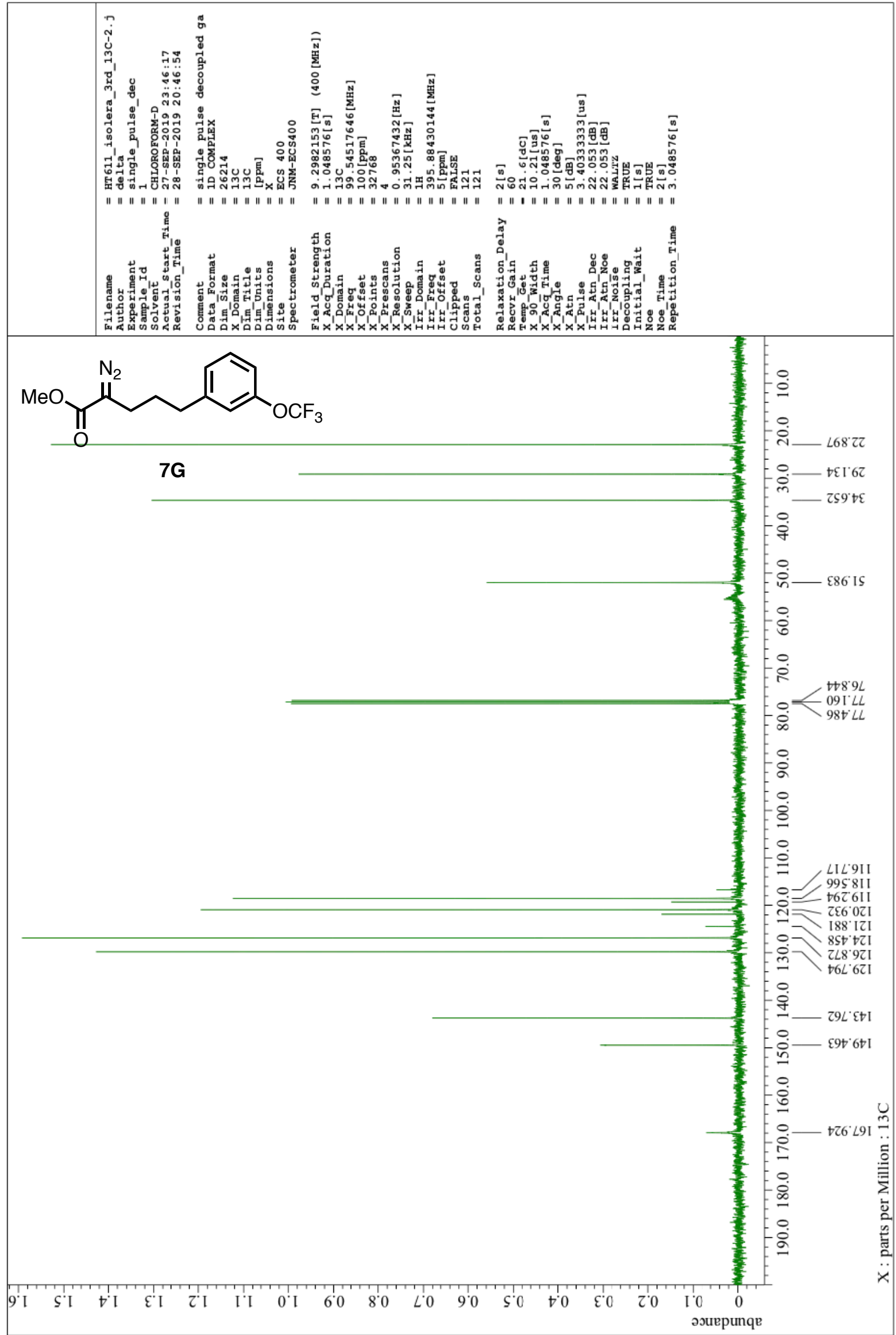


${ }^{1} \mathrm{H}$ NMR of $7 \mathbf{H}\left(400 \mathrm{MHz}, \mathrm{CDCl}_{3}\right)$

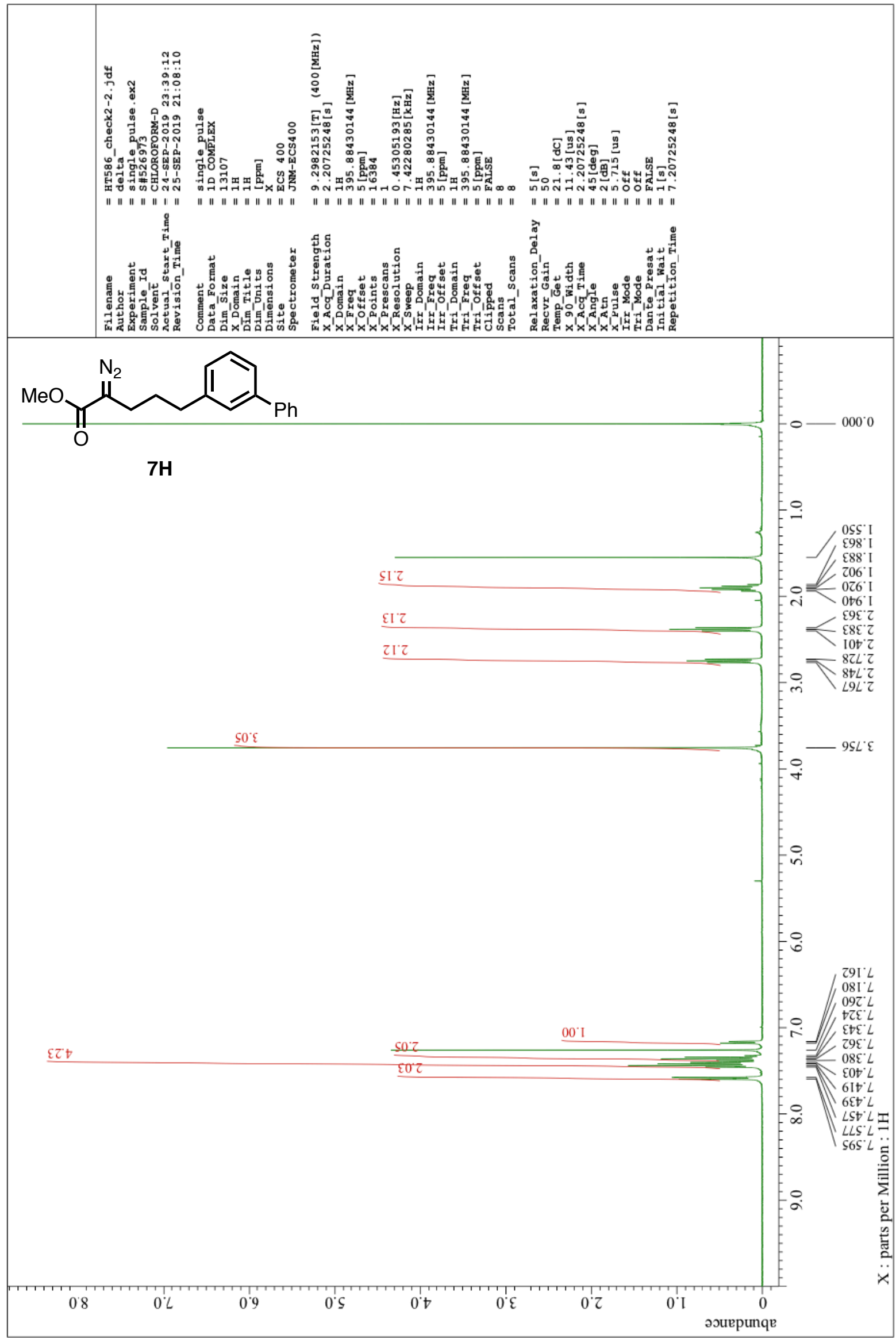


${ }^{13} \mathrm{C}$ NMR of $7 \mathbf{H}\left(101 \mathrm{MHz}, \mathrm{CDCl}_{3}\right)$

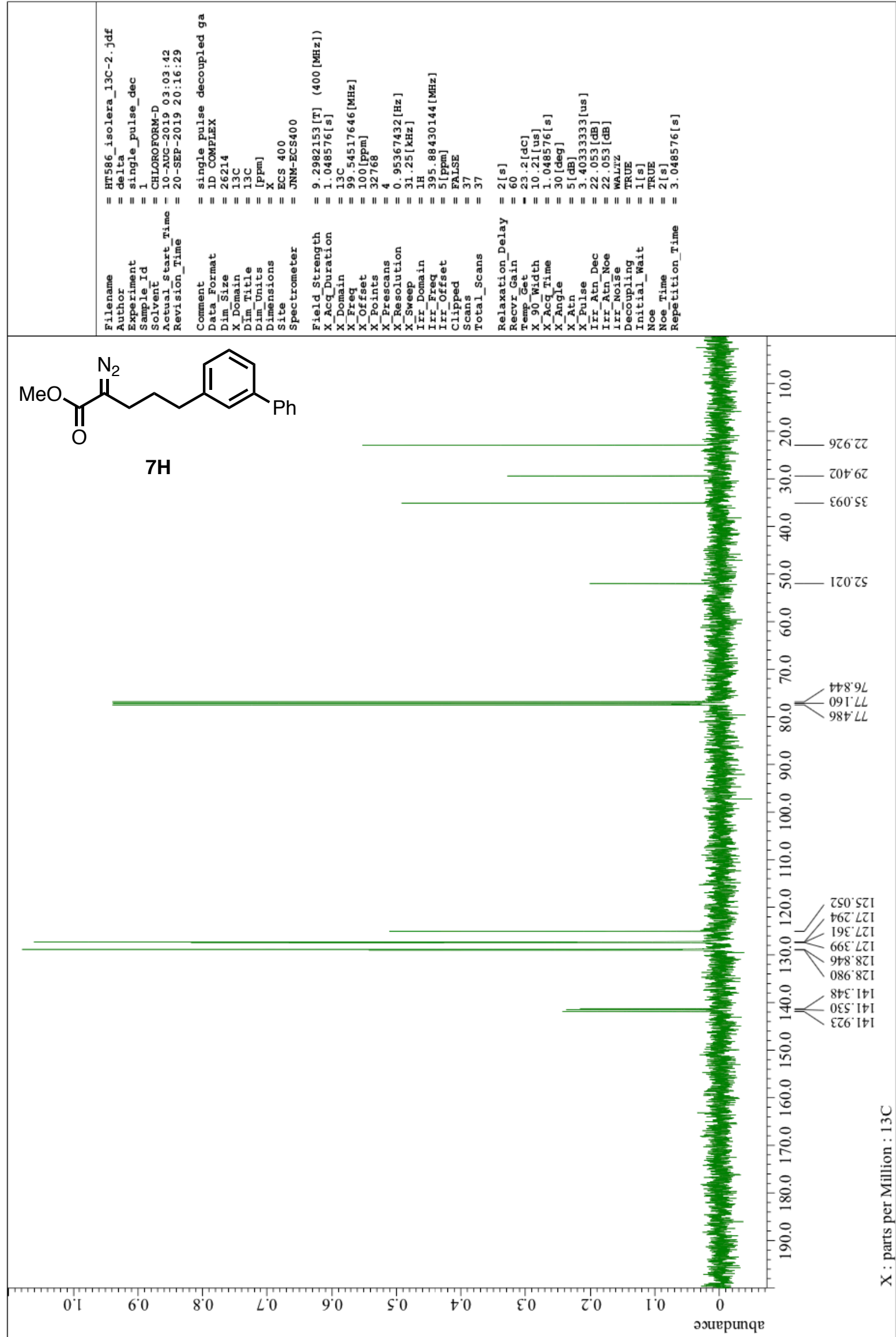


${ }^{1} \mathrm{H}$ NMR of $7 \mathbf{I}\left(400 \mathrm{MHz}, \mathrm{CDCl}_{3}\right)$

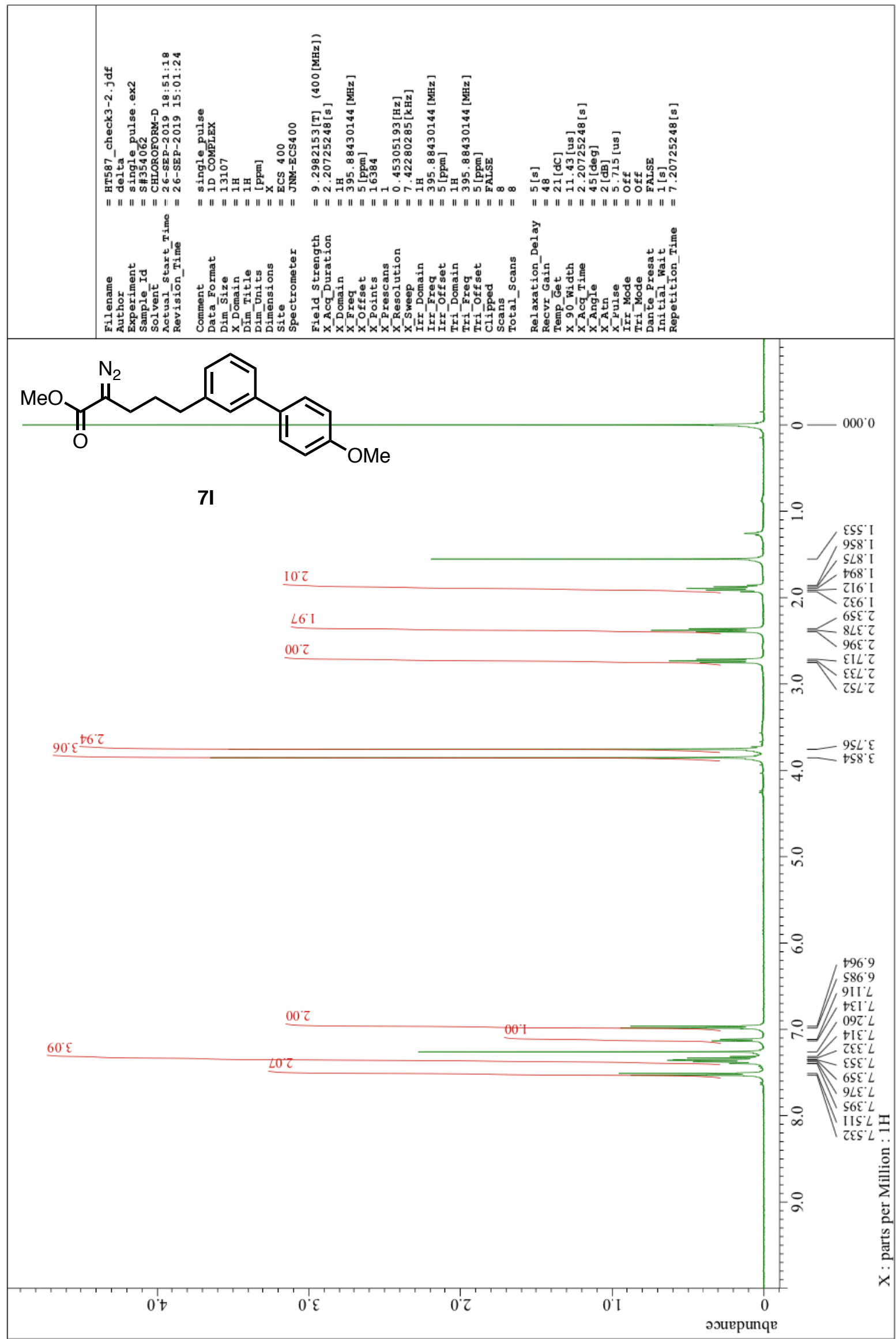


${ }^{13} \mathrm{C}$ NMR of $7 \mathbf{I}\left(101 \mathrm{MHz}, \mathrm{CDCl}_{3}\right)$

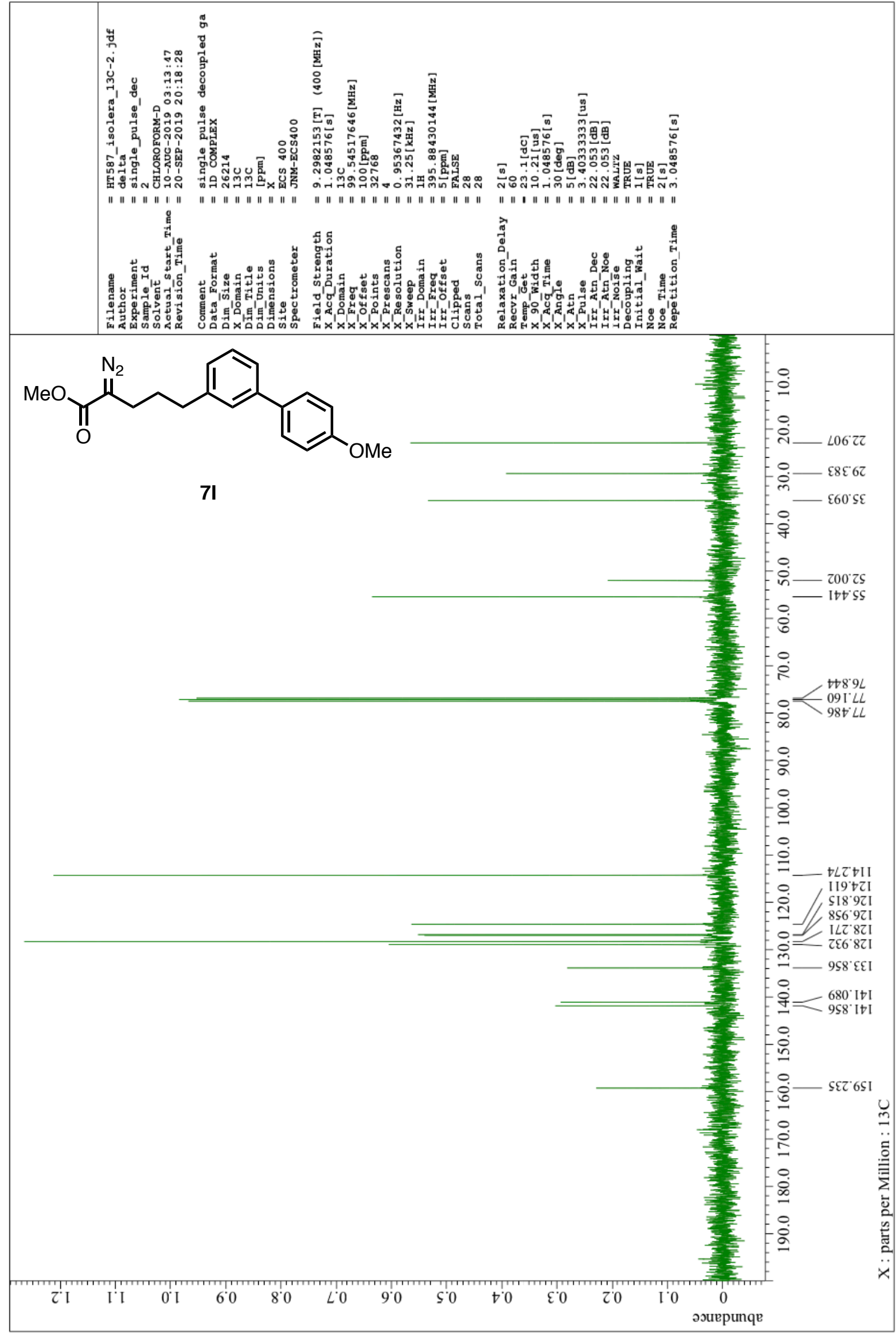


${ }^{1} \mathrm{H} \mathrm{NMR}$ of $7 \mathbf{J}\left(400 \mathrm{MHz}, \mathrm{CDCl}_{3}\right)$

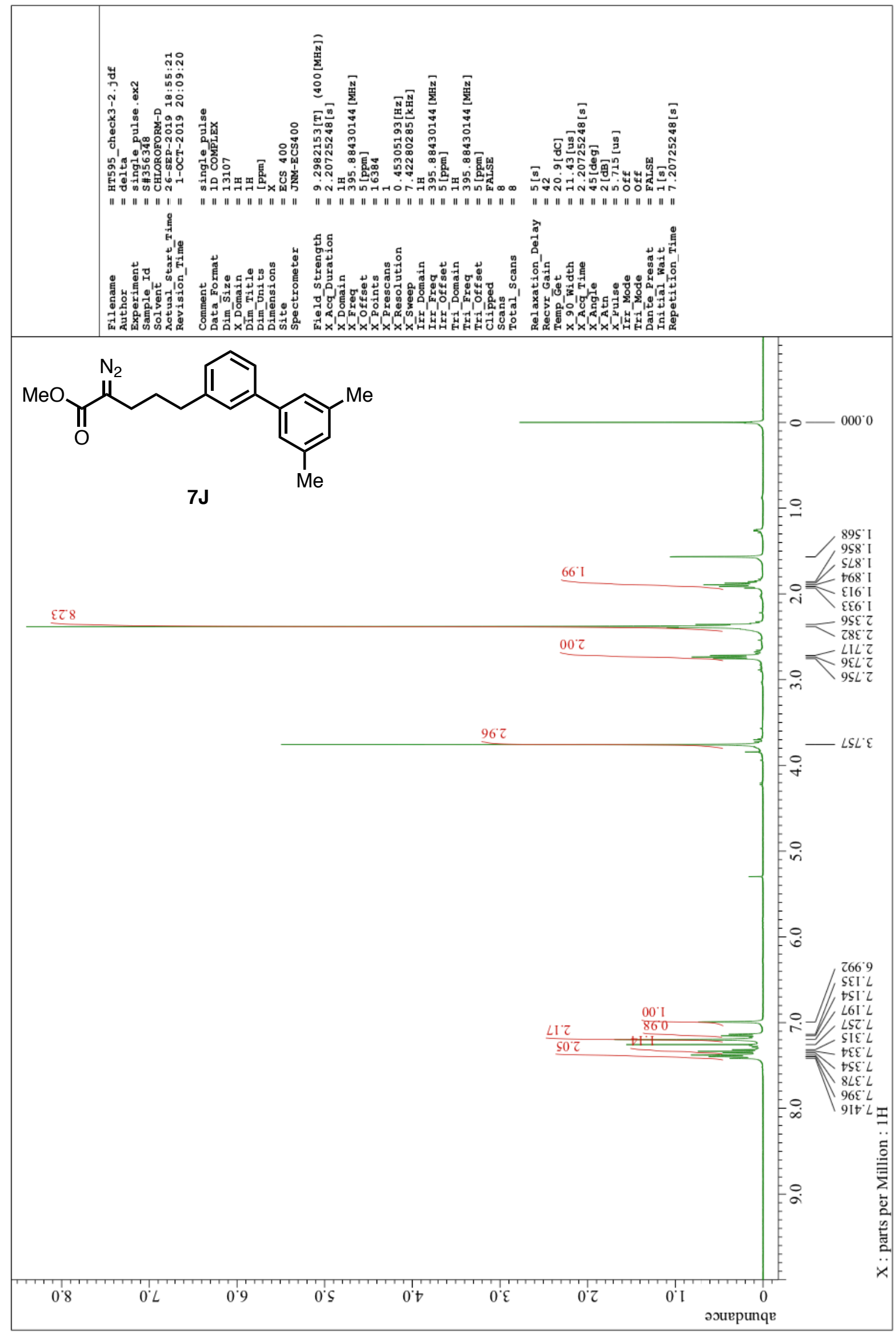


${ }^{13} \mathrm{C}$ NMR of $7 \mathbf{J}\left(101 \mathrm{MHz}, \mathrm{CDCl}_{3}\right)$

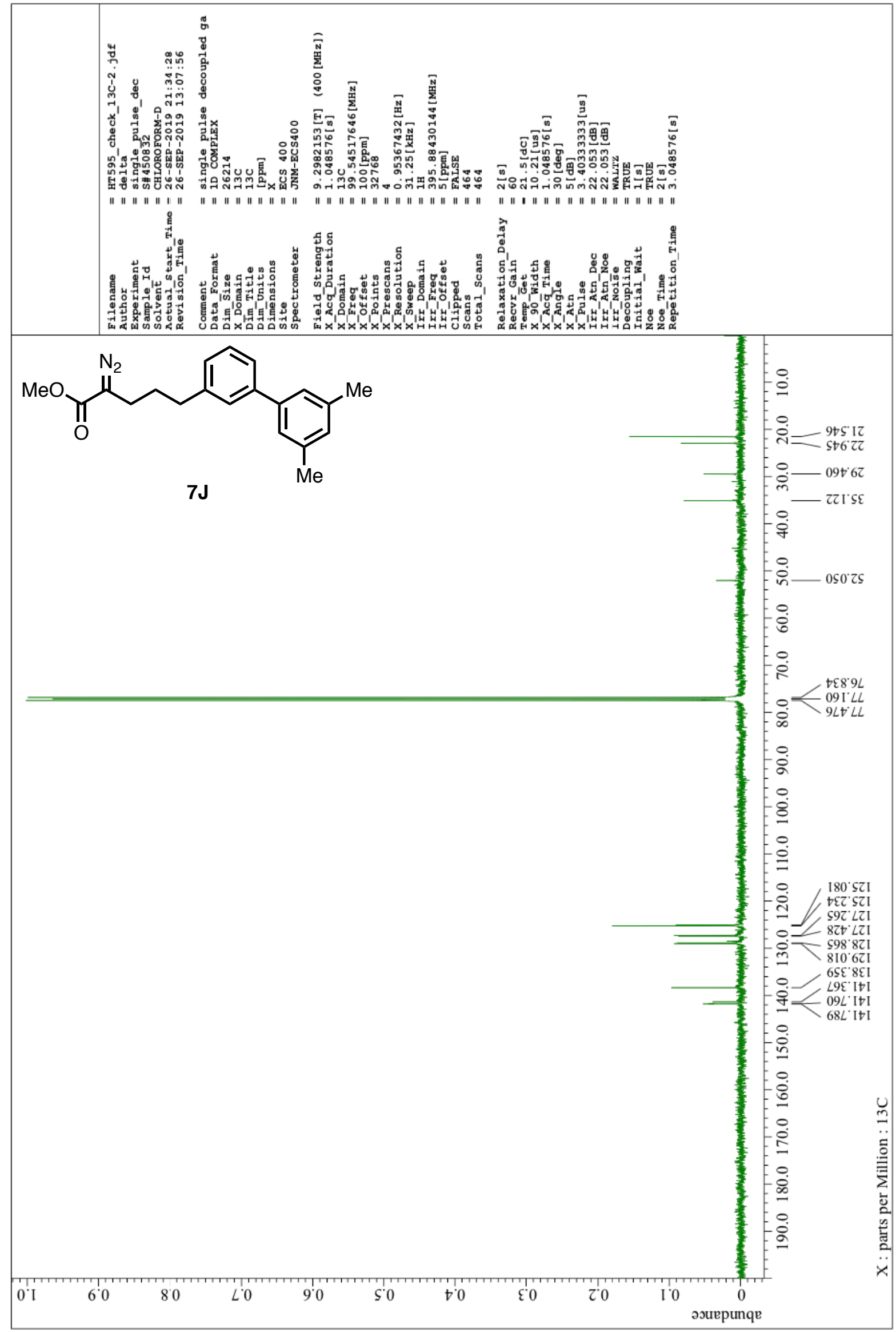


${ }^{1} \mathrm{H}$ NMR of $7 \mathbf{K}\left(400 \mathrm{MHz}, \mathrm{CDCl}_{3}\right)$

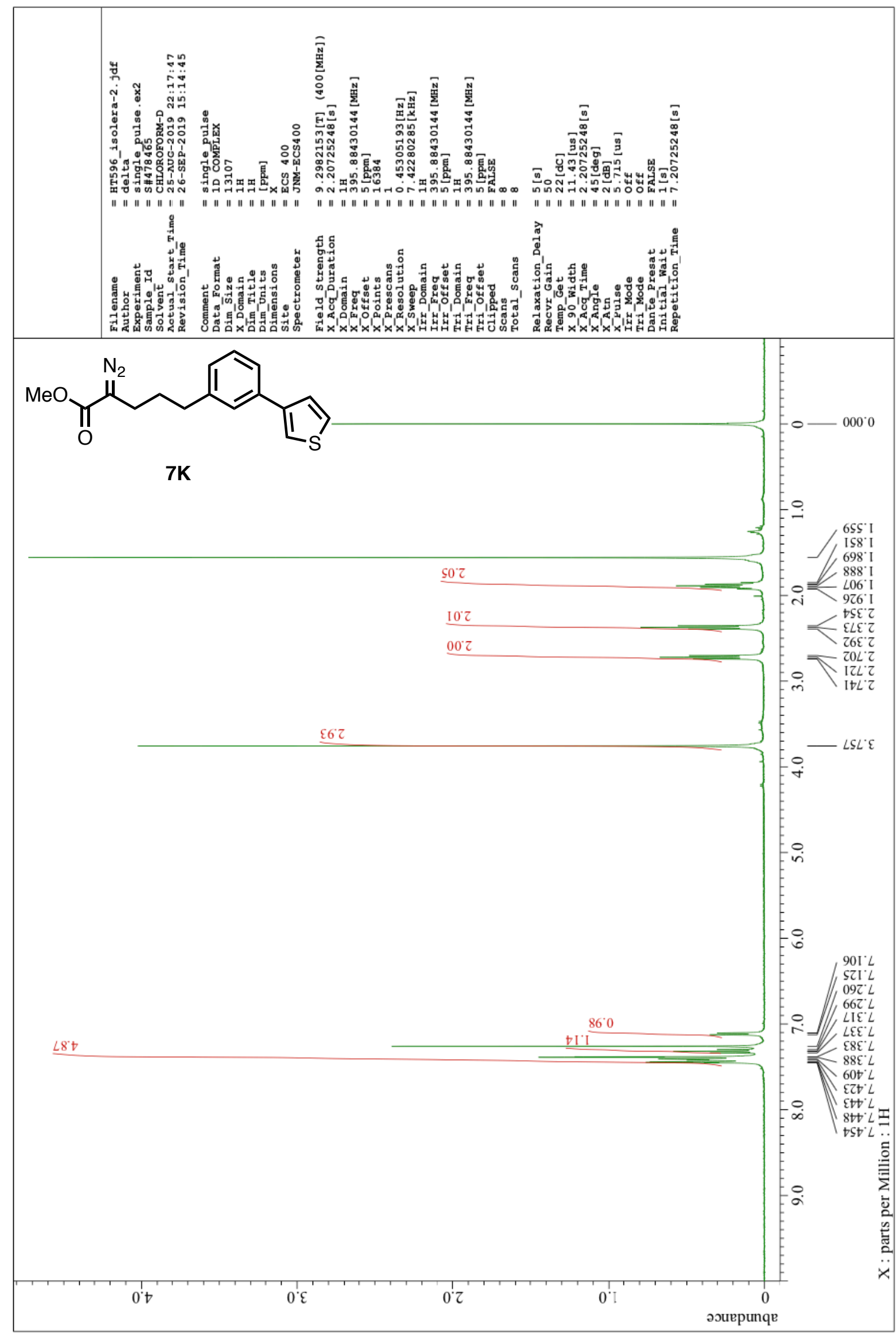


${ }^{13} \mathrm{C}$ NMR of $7 \mathbf{K}\left(101 \mathrm{MHz}, \mathrm{CDCl}_{3}\right)$

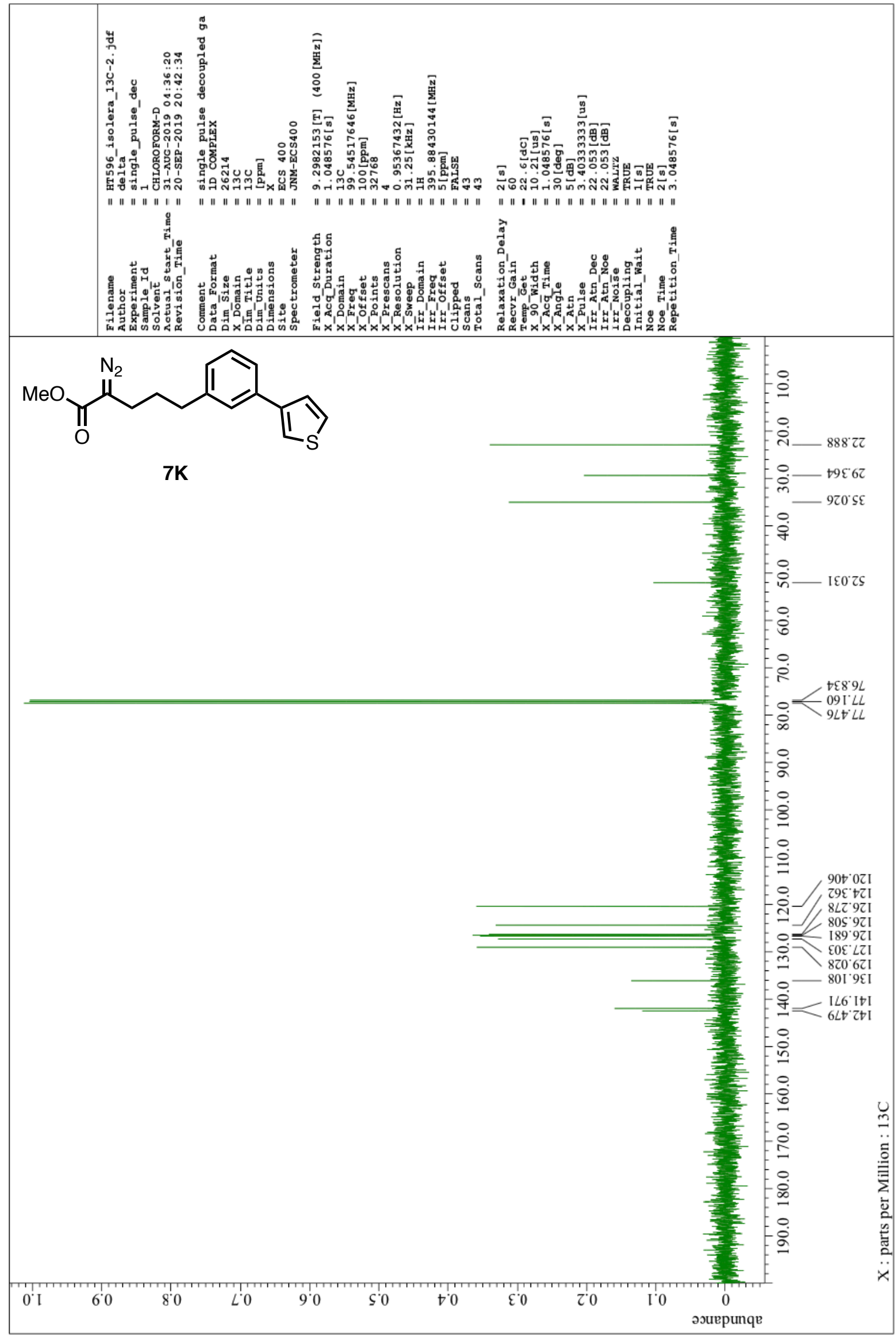


${ }^{1} \mathrm{H}$ NMR of $2 \mathrm{~A}\left(400 \mathrm{MHz}, \mathrm{CDCl}_{3}\right)$

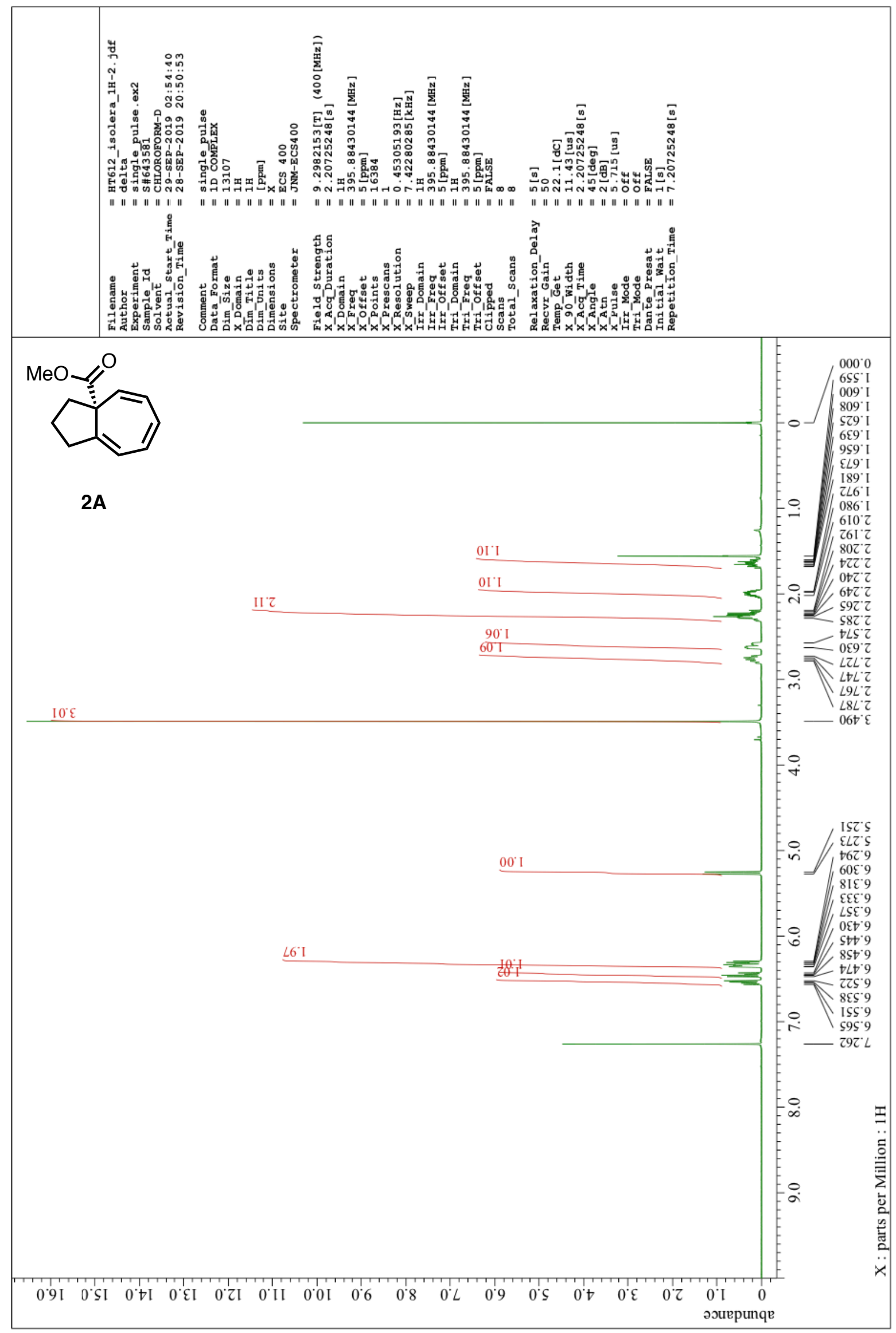


${ }^{13} \mathrm{C}$ NMR of $\mathbf{2 A}\left(101 \mathrm{MHz}, \mathrm{CDCl}_{3}\right)$

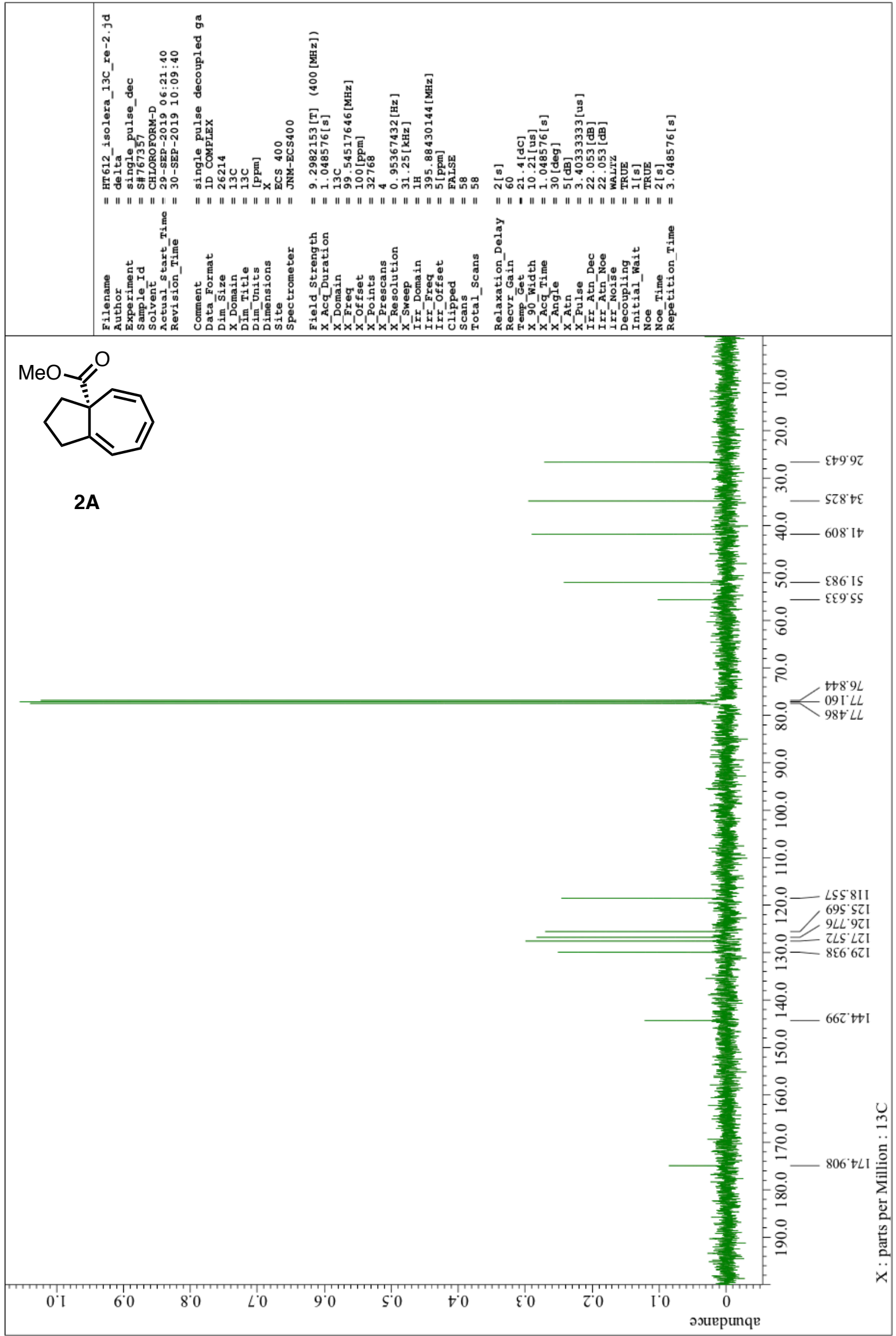


${ }^{1} \mathrm{H}$ NMR of $2 \mathbf{B}\left(400 \mathrm{MHz}, \mathrm{CDCl}_{3}\right)$

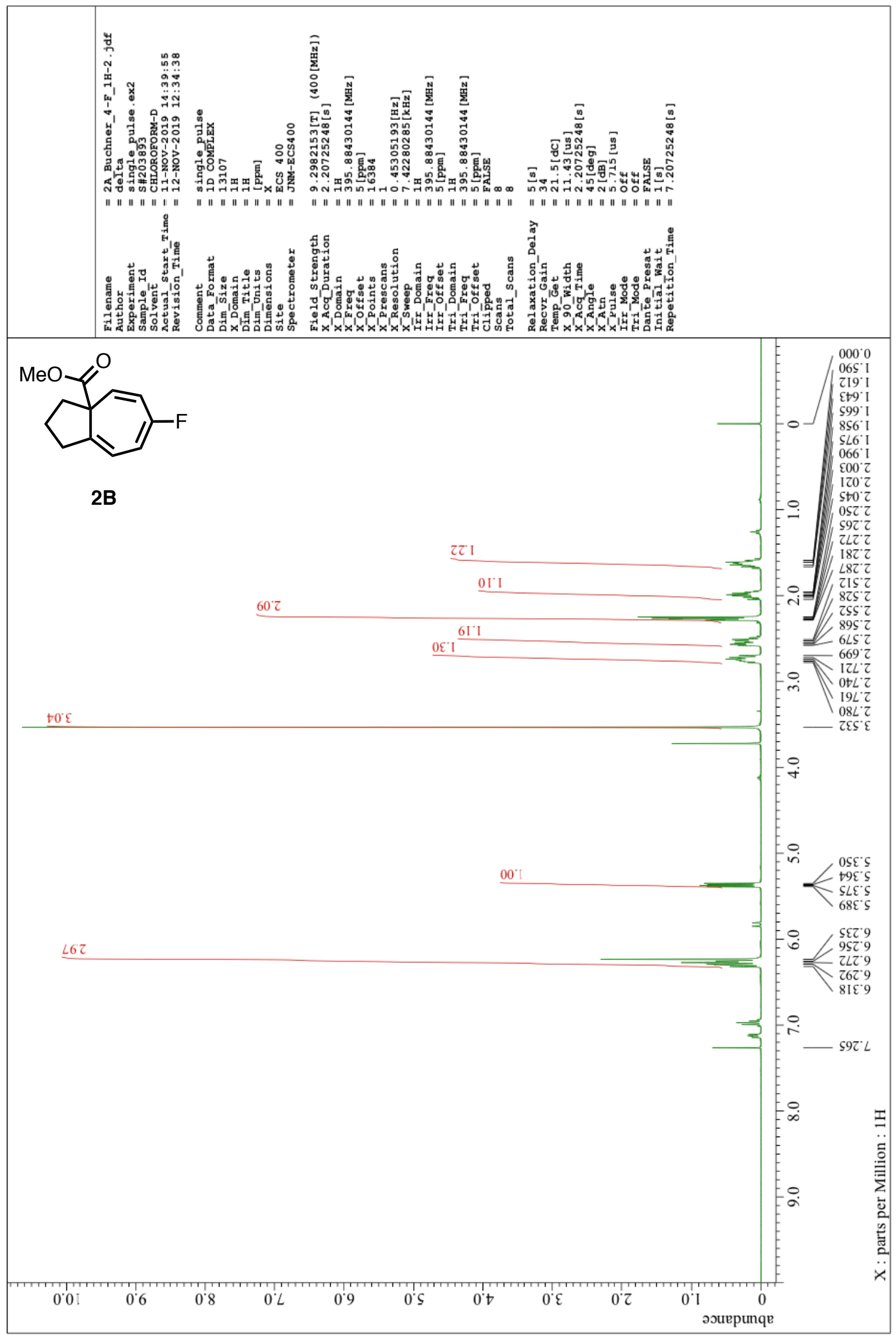


${ }^{13} \mathrm{C}$ NMR of 2B $\left(101 \mathrm{MHz}, \mathrm{CDCl}_{3}\right)$

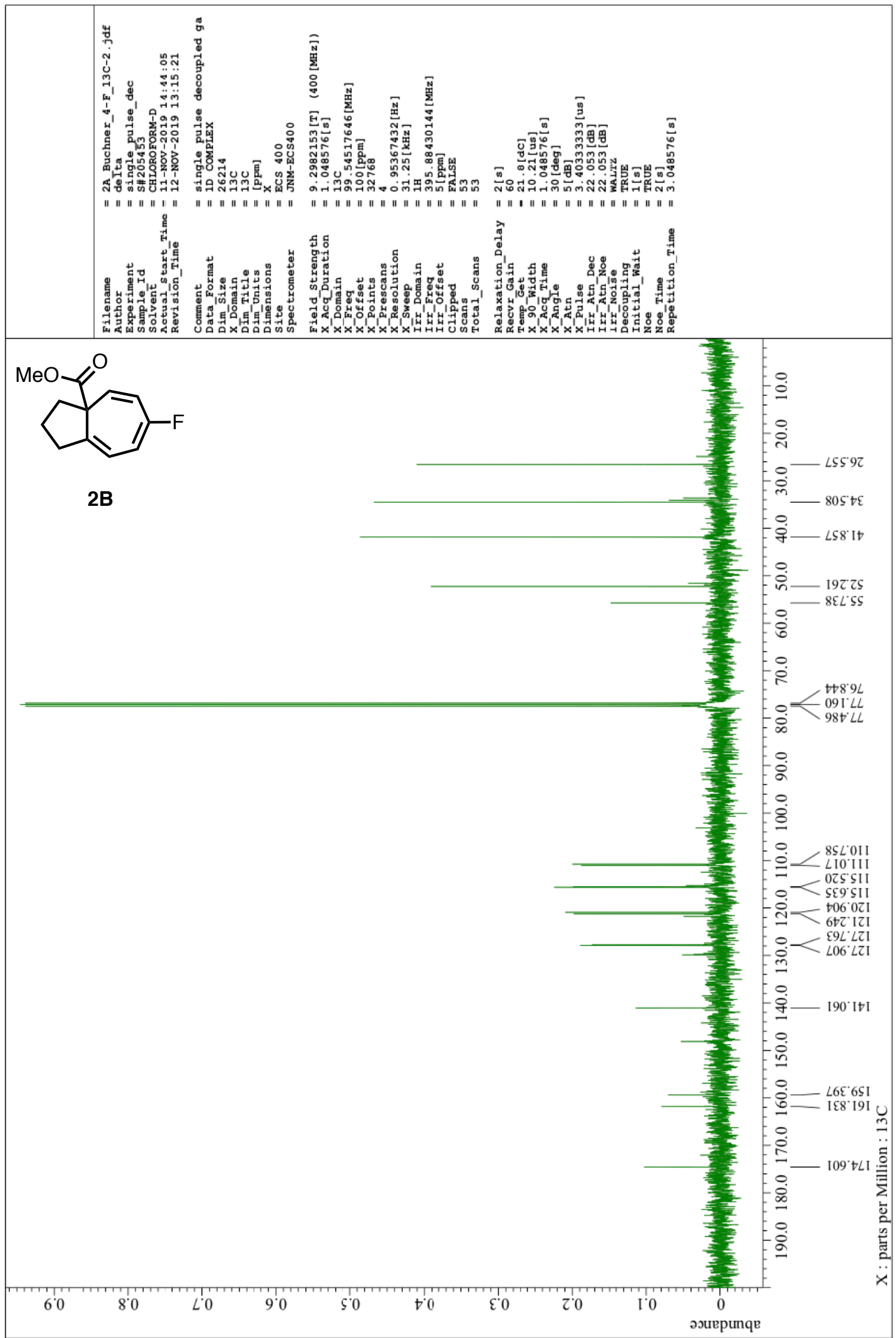


${ }^{1} \mathrm{H}$ NMR of $2 \mathrm{C}\left(400 \mathrm{MHz}, \mathrm{CDCl}_{3}\right)$

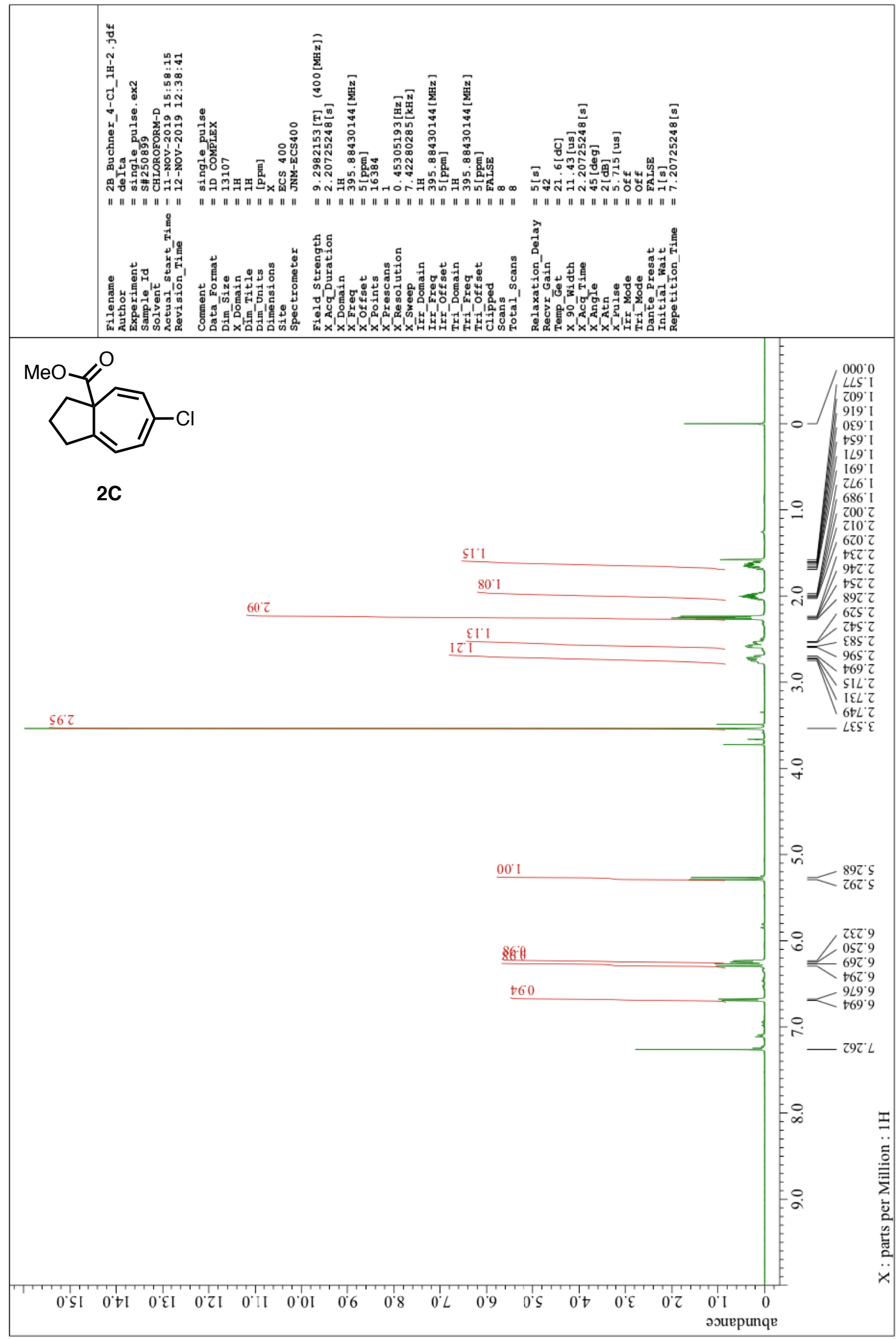


${ }^{13} \mathrm{C}$ NMR of $2 \mathrm{C}\left(101 \mathrm{MHz}, \mathrm{CDCl}_{3}\right)$

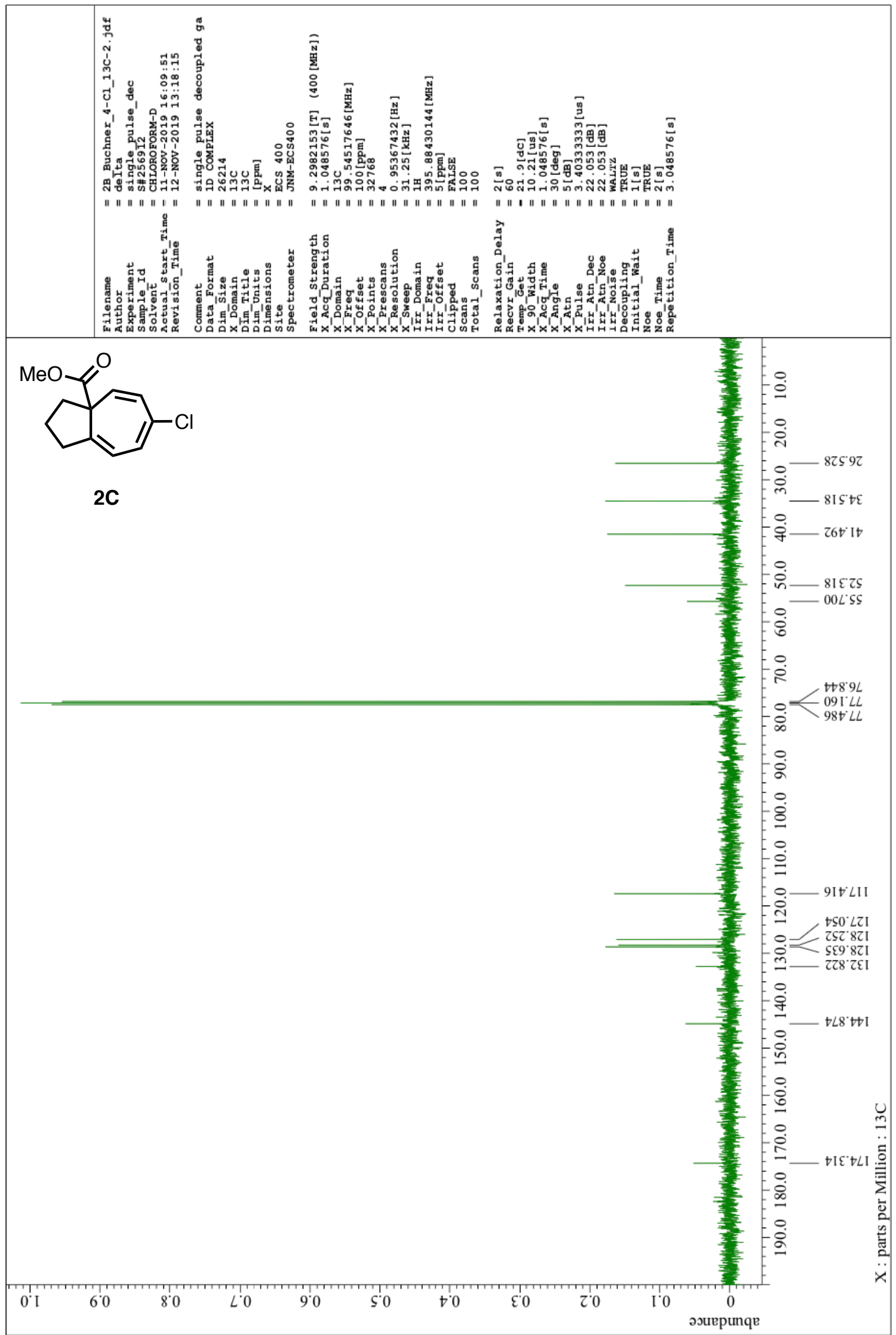


${ }^{1} \mathrm{H}$ NMR of $2 D\left(400 \mathrm{MHz}, \mathrm{CDCl}_{3}\right)$

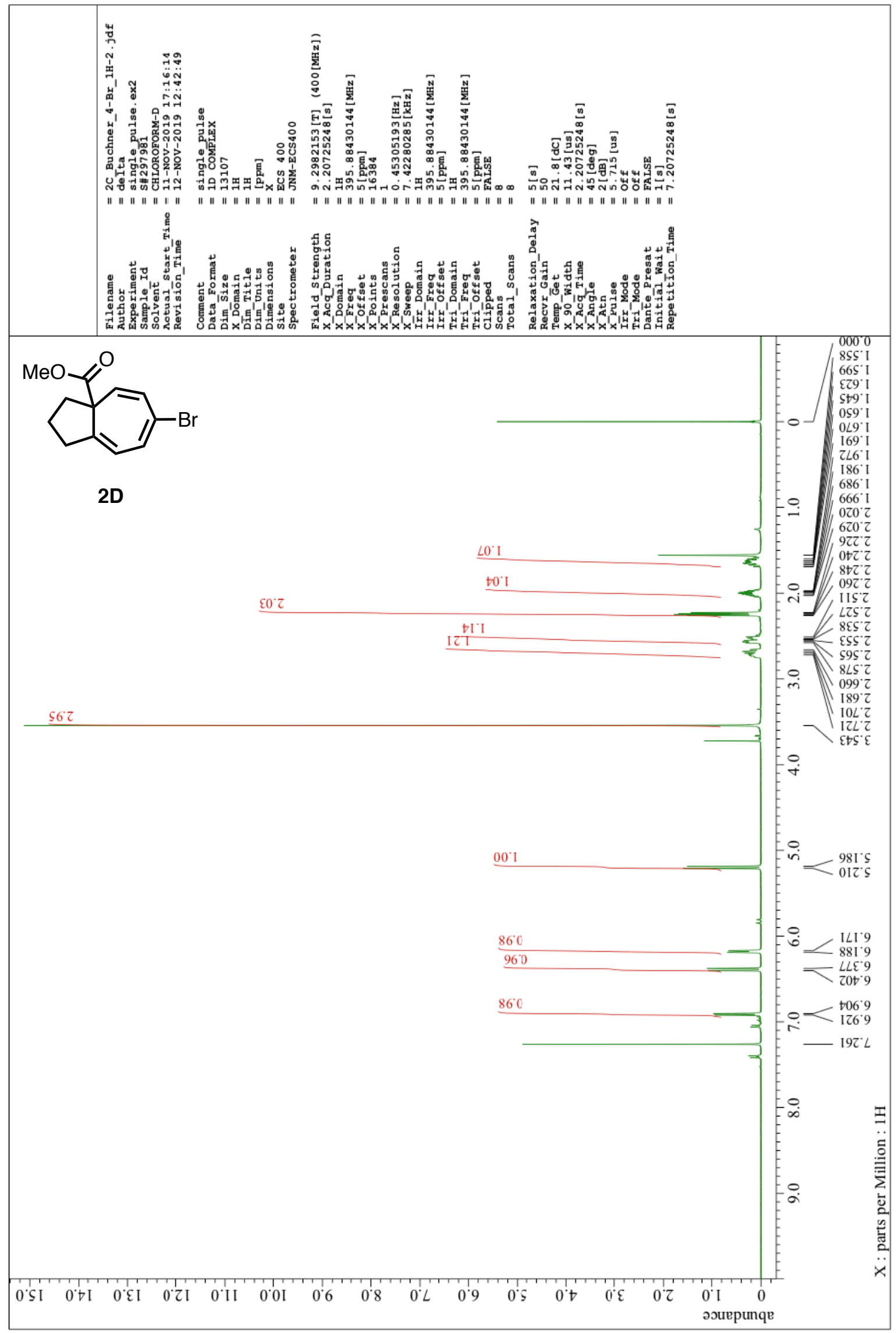


${ }^{13} \mathrm{C}$ NMR of 2D $\left(101 \mathrm{MHz}, \mathrm{CDCl}_{3}\right)$

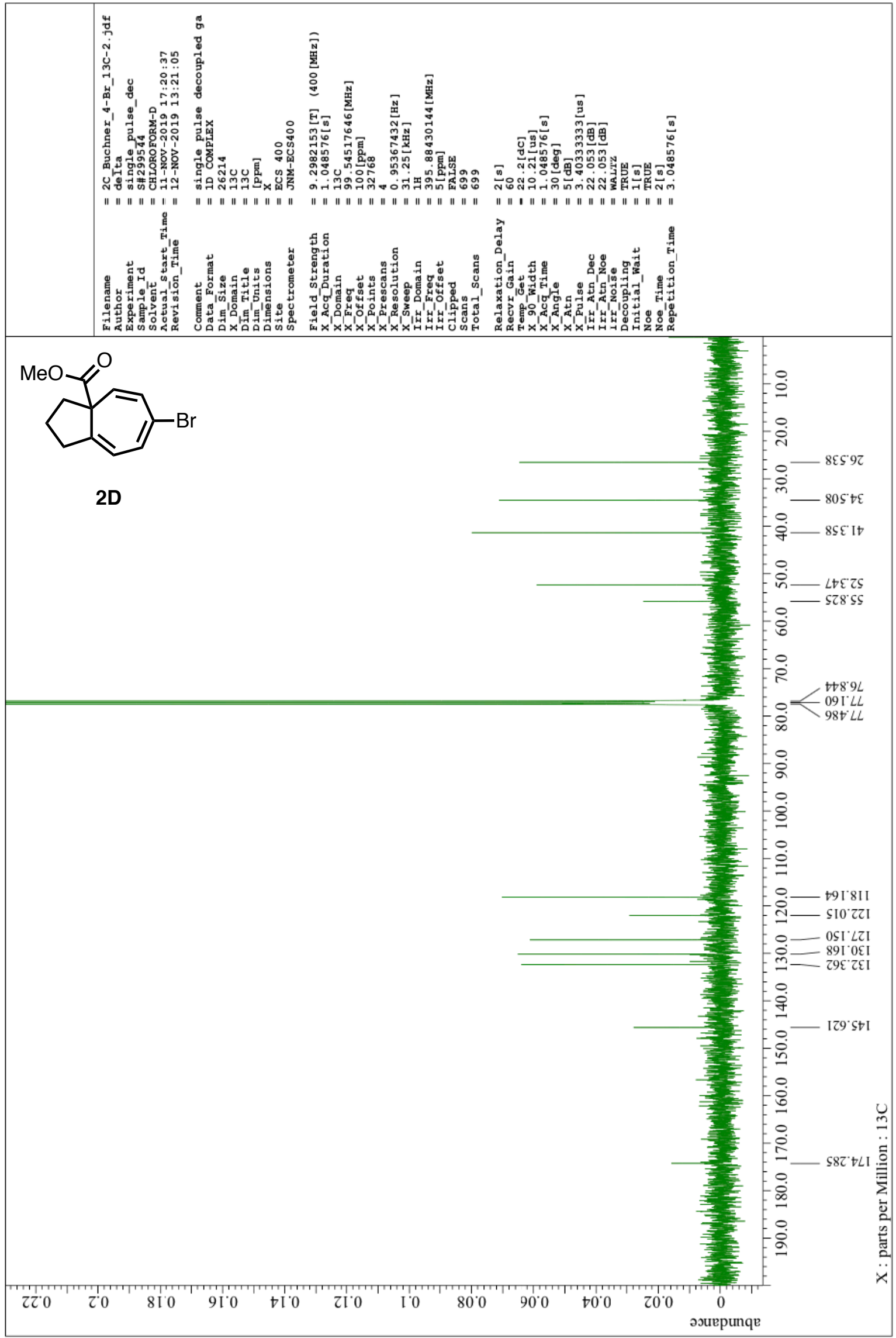


${ }^{1} \mathrm{H}$ NMR of $2 \mathbf{E}\left(400 \mathrm{MHz}, \mathrm{CDCl}_{3}\right)$

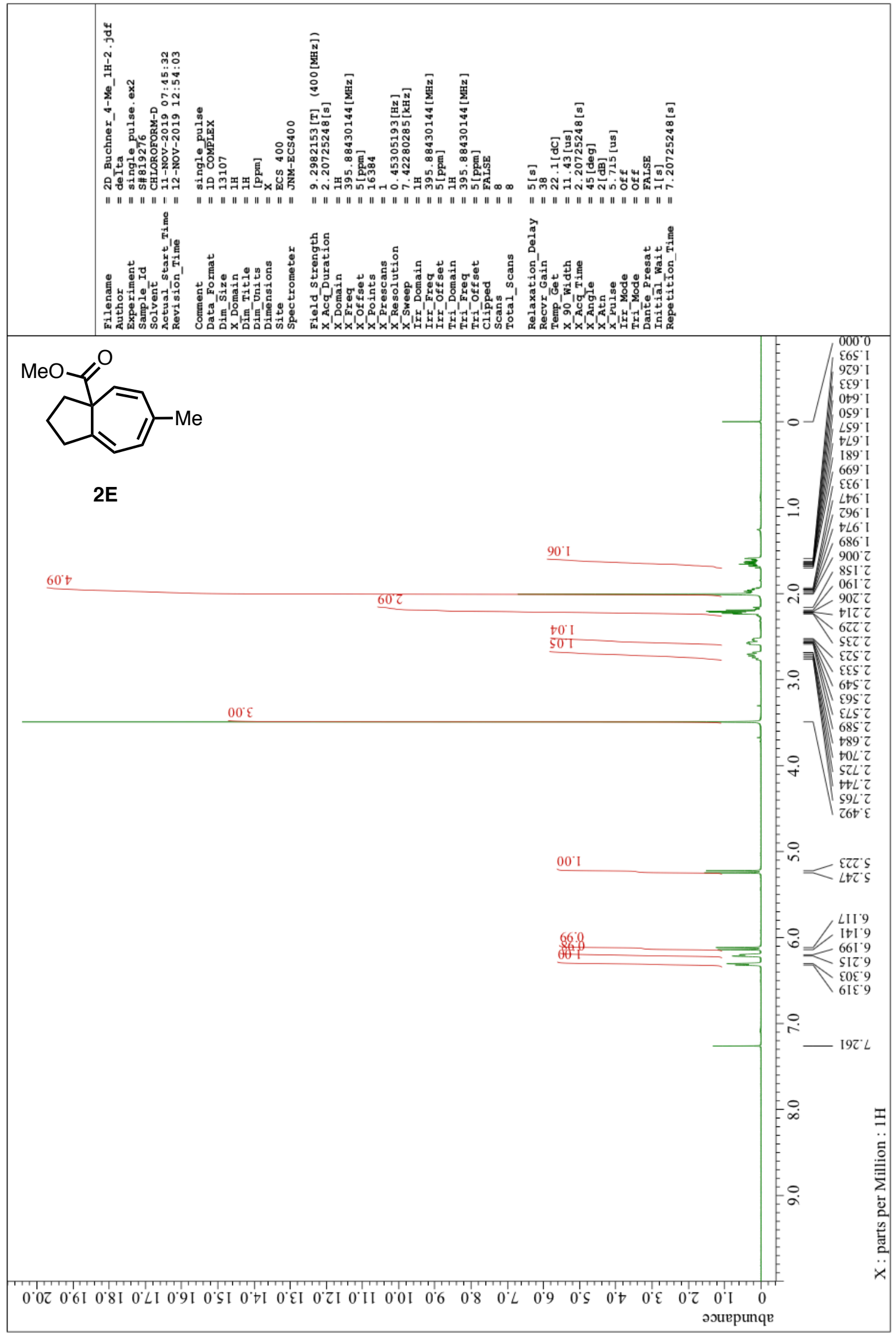


${ }^{13} \mathrm{C}$ NMR of $2 \mathbf{E}\left(101 \mathrm{MHz}, \mathrm{CDCl}_{3}\right)$

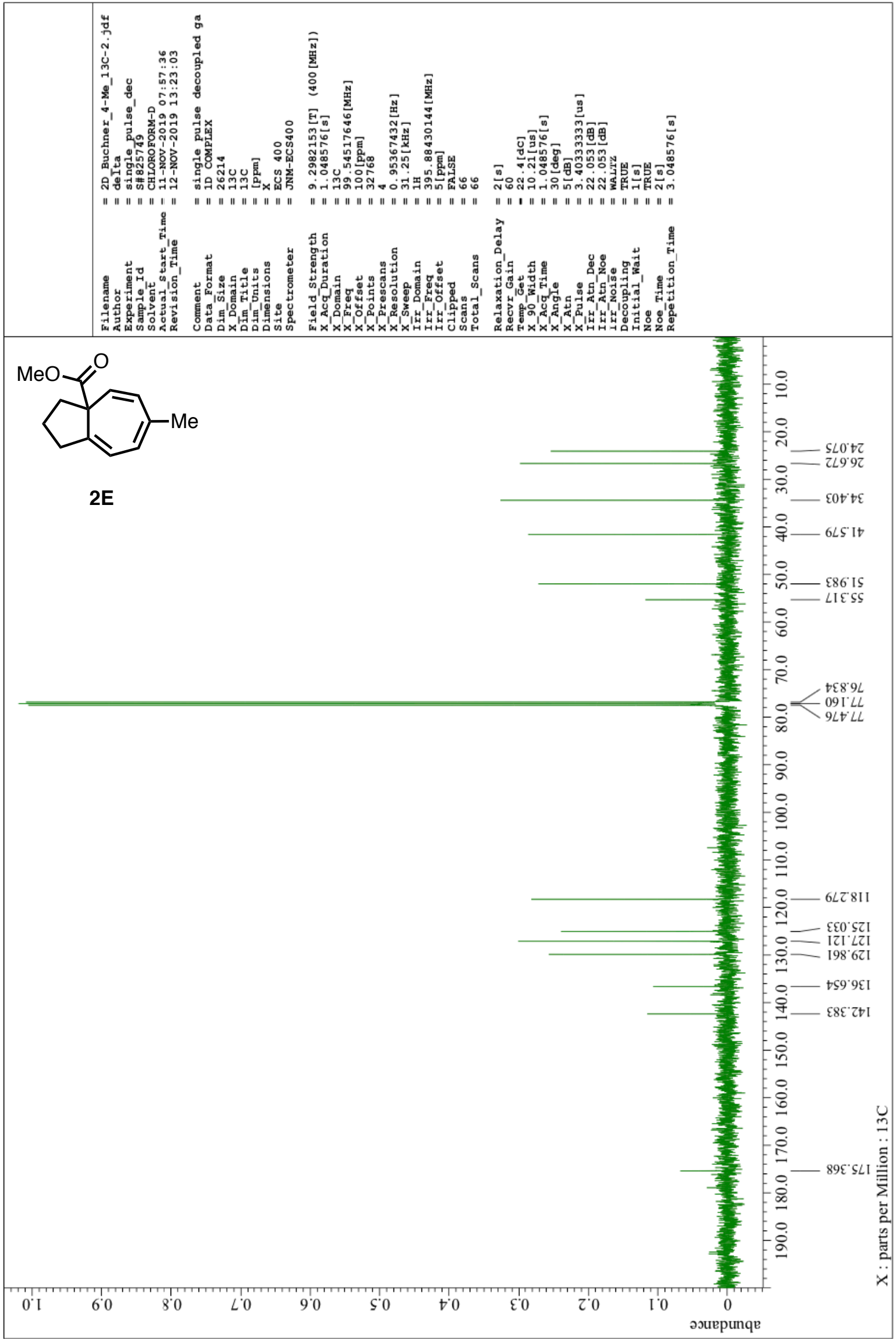


${ }^{1} \mathrm{H}$ NMR of $2 \mathbf{F}\left(400 \mathrm{MHz}, \mathrm{CDCl}_{3}\right)$

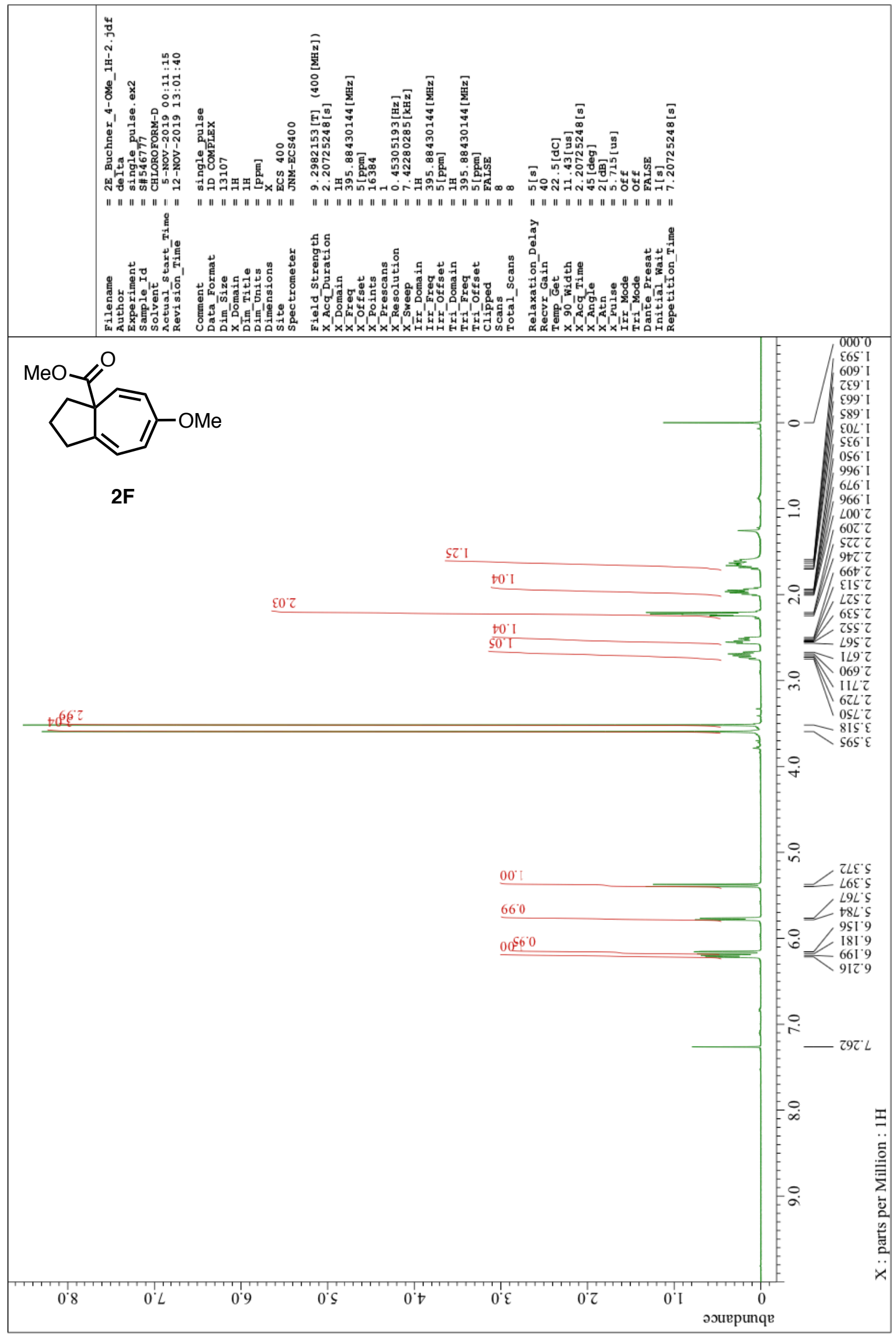


${ }^{13} \mathrm{C}$ NMR of $\mathbf{2 F}\left(101 \mathrm{MHz}, \mathrm{CDCl}_{3}\right)$

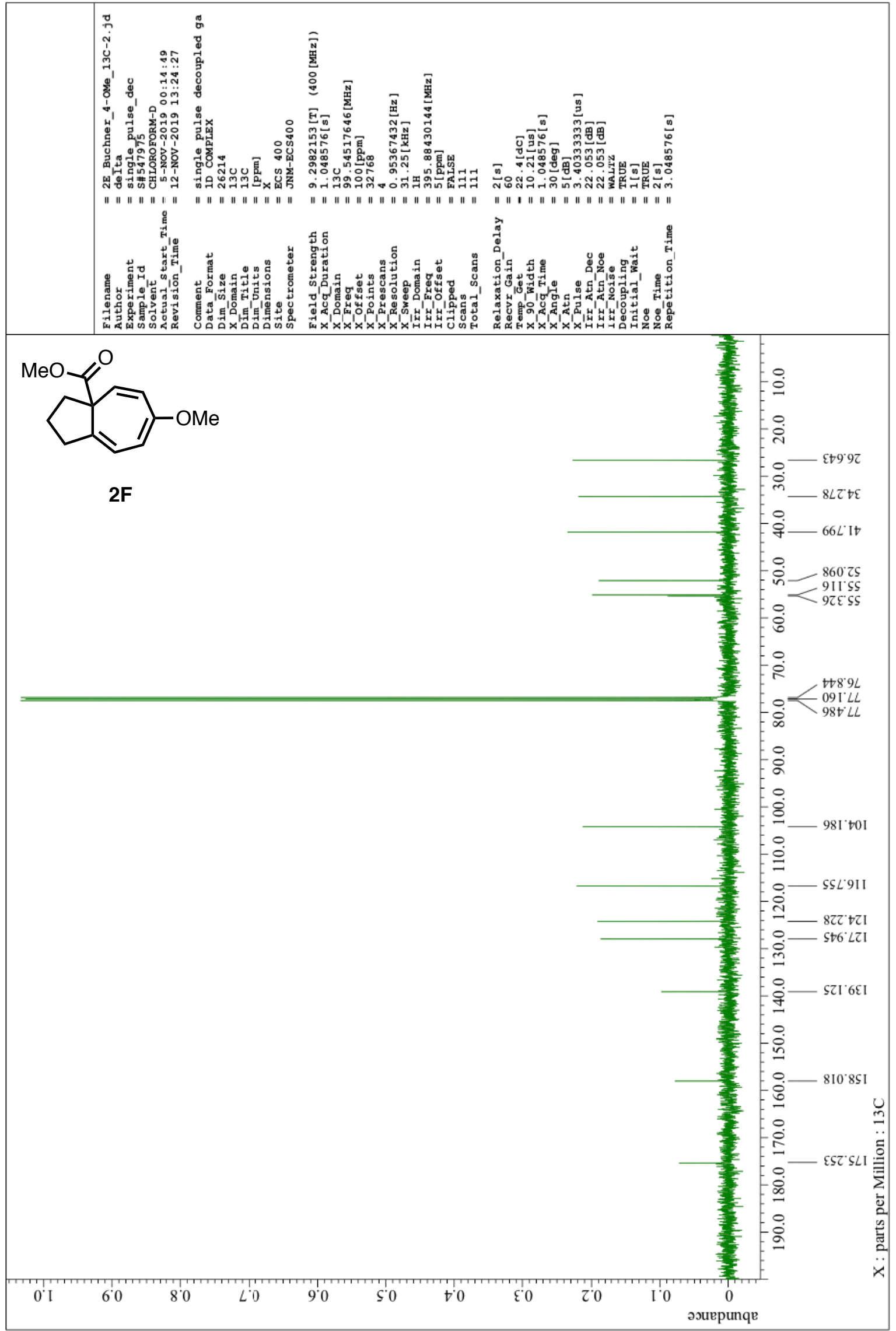


${ }^{1} \mathrm{H}$ NMR of $5\left(400 \mathrm{MHz}, \mathrm{CDCl}_{3}\right)$

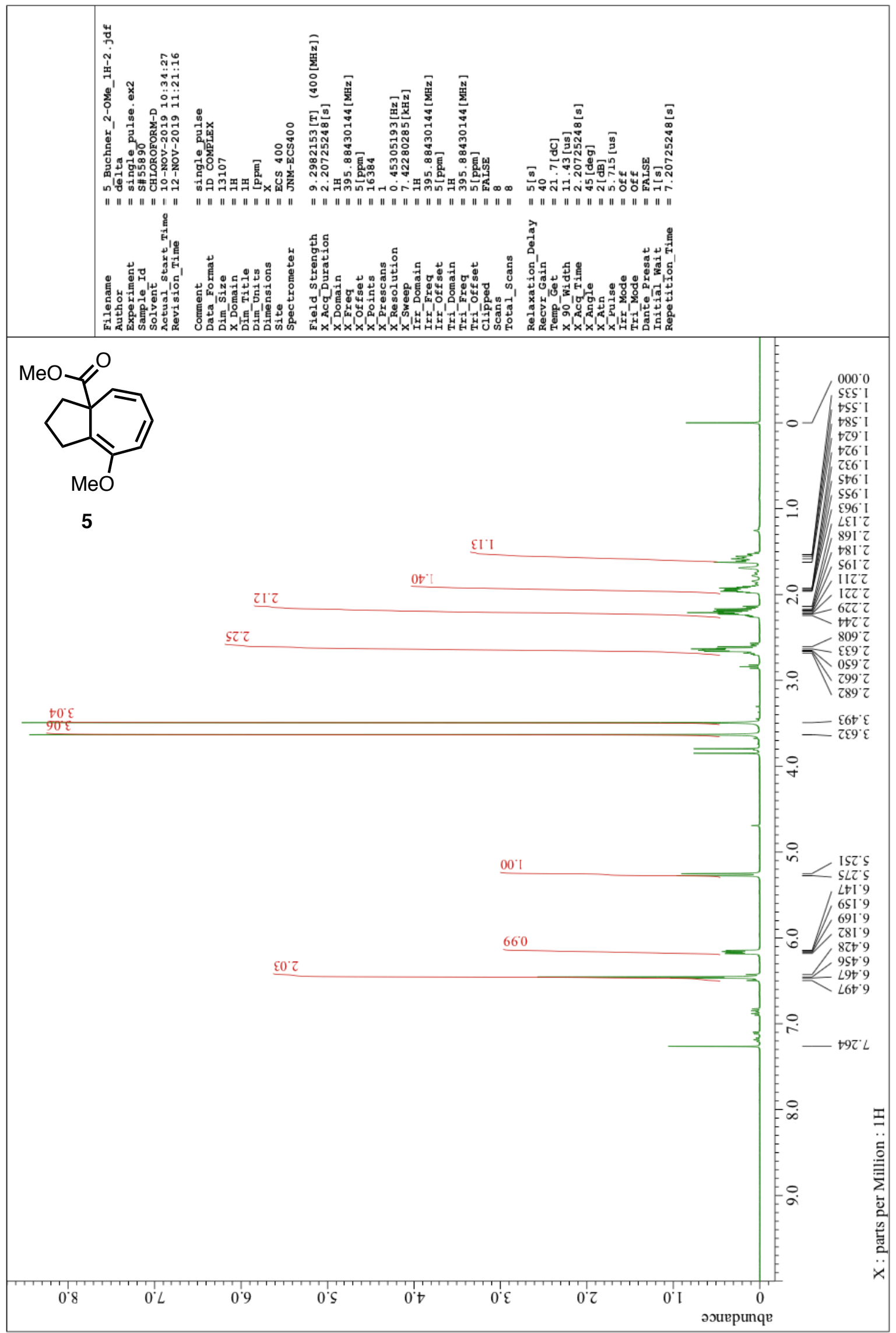


${ }^{13} \mathrm{C}$ NMR of $5\left(101 \mathrm{MHz}, \mathrm{CDCl}_{3}\right)$

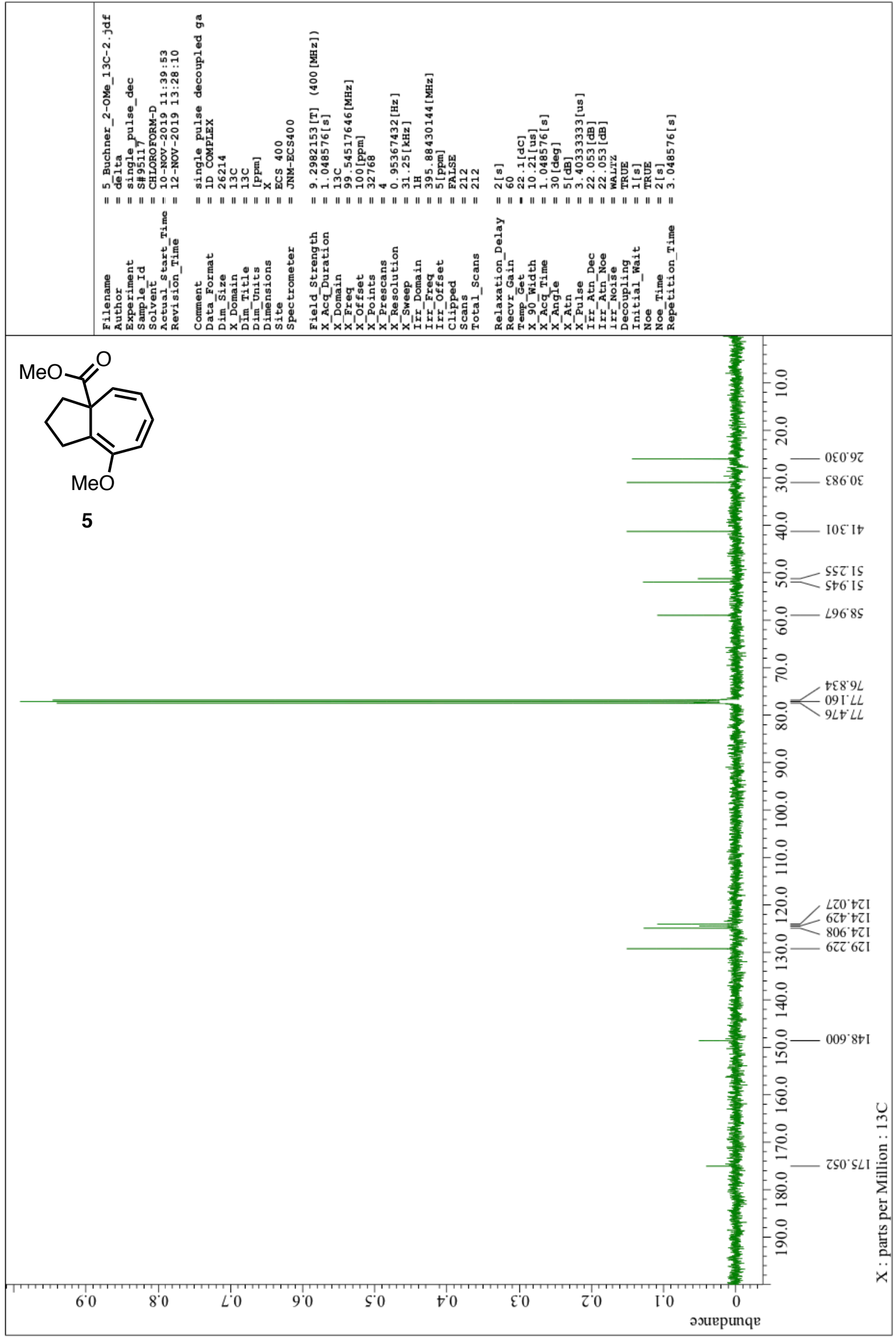


${ }^{1} \mathrm{H}$ NMR of $\mathbf{8 A}\left(400 \mathrm{MHz}, \mathrm{CDCl}_{3}\right)$

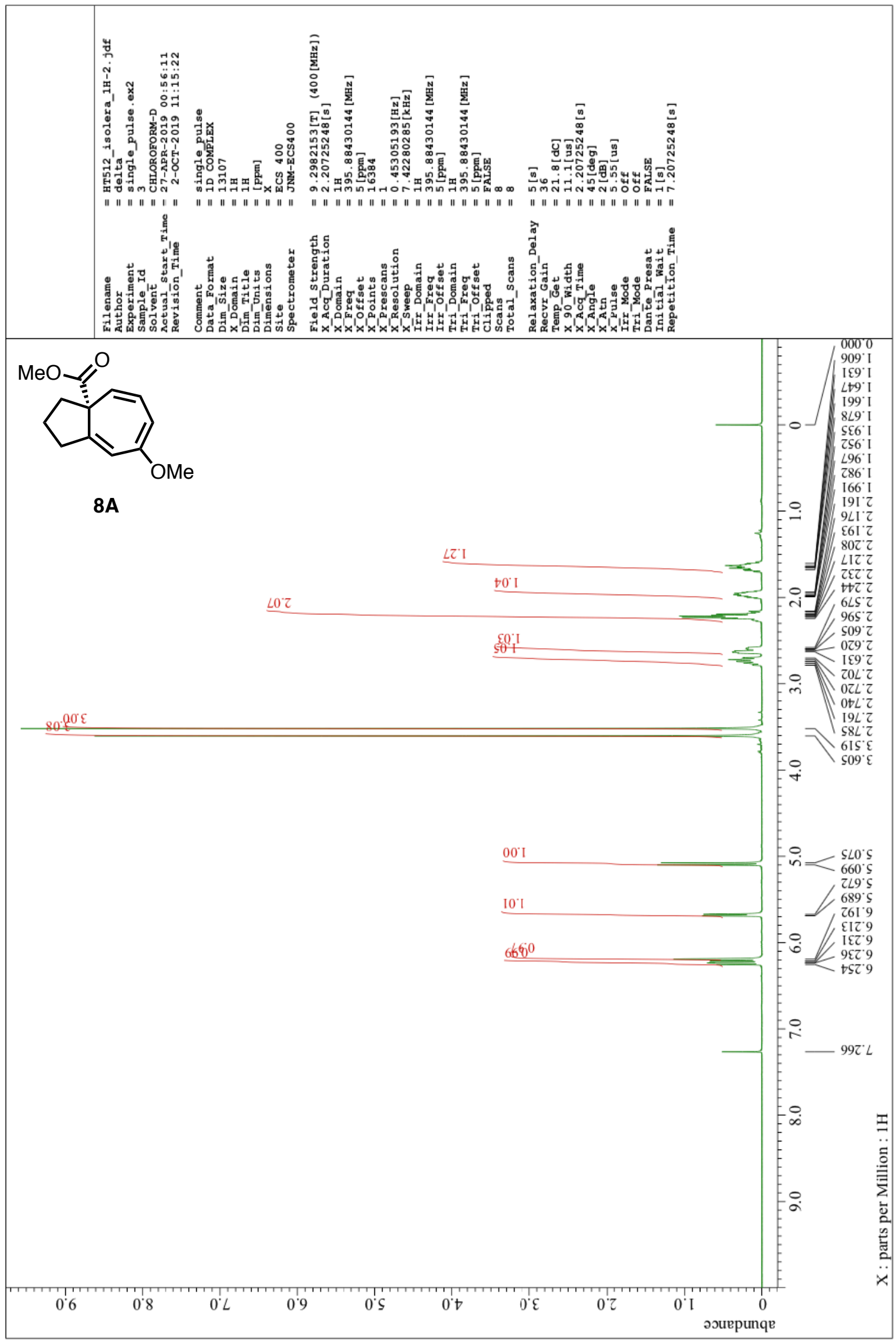


${ }^{13} \mathrm{C}$ NMR of $\mathbf{8 A}\left(101 \mathrm{MHz}, \mathrm{CDCl}_{3}\right)$

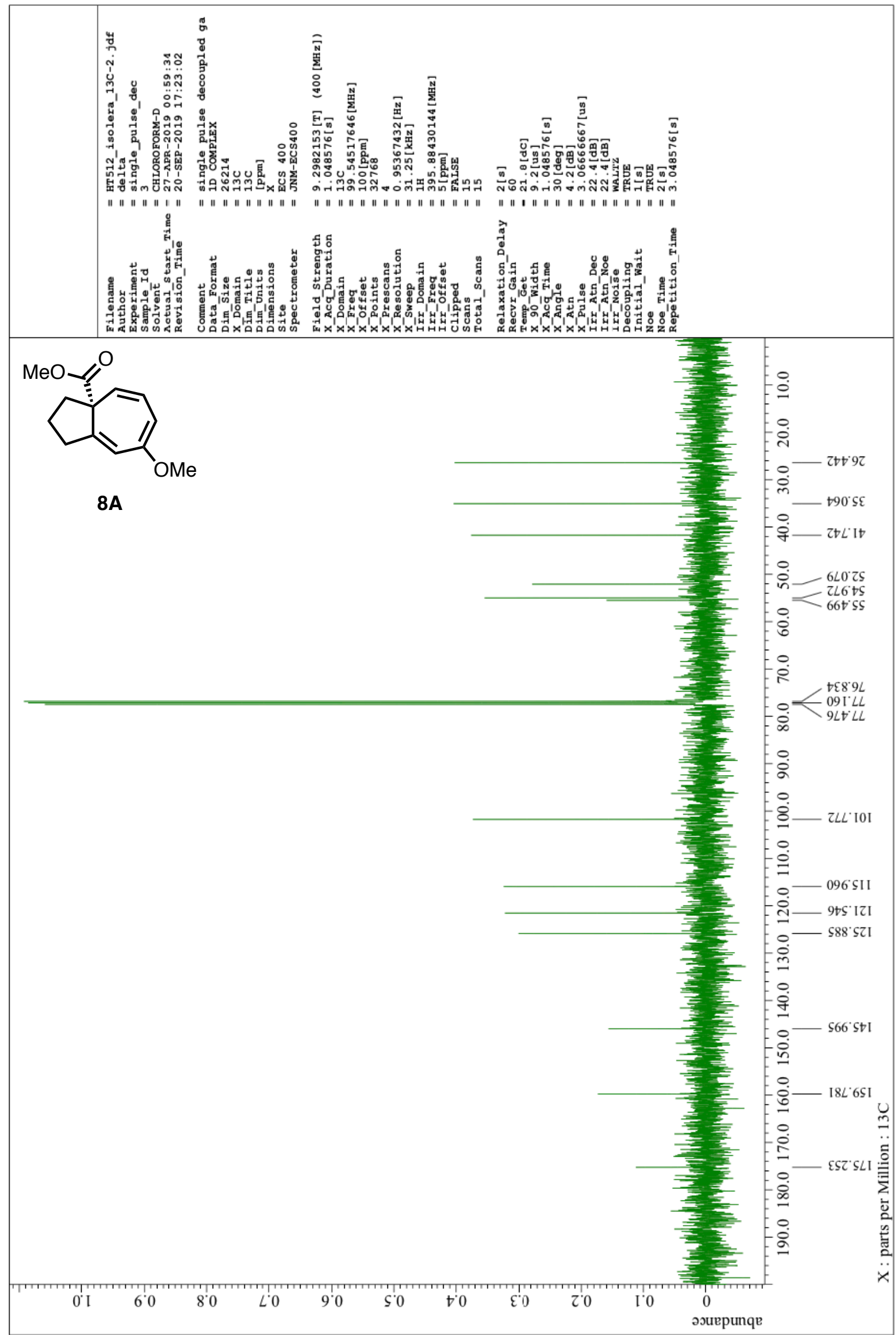


${ }^{1} \mathrm{H}$ NMR of $\mathbf{8 B}\left(400 \mathrm{MHz}, \mathrm{CDCl}_{3}\right)$

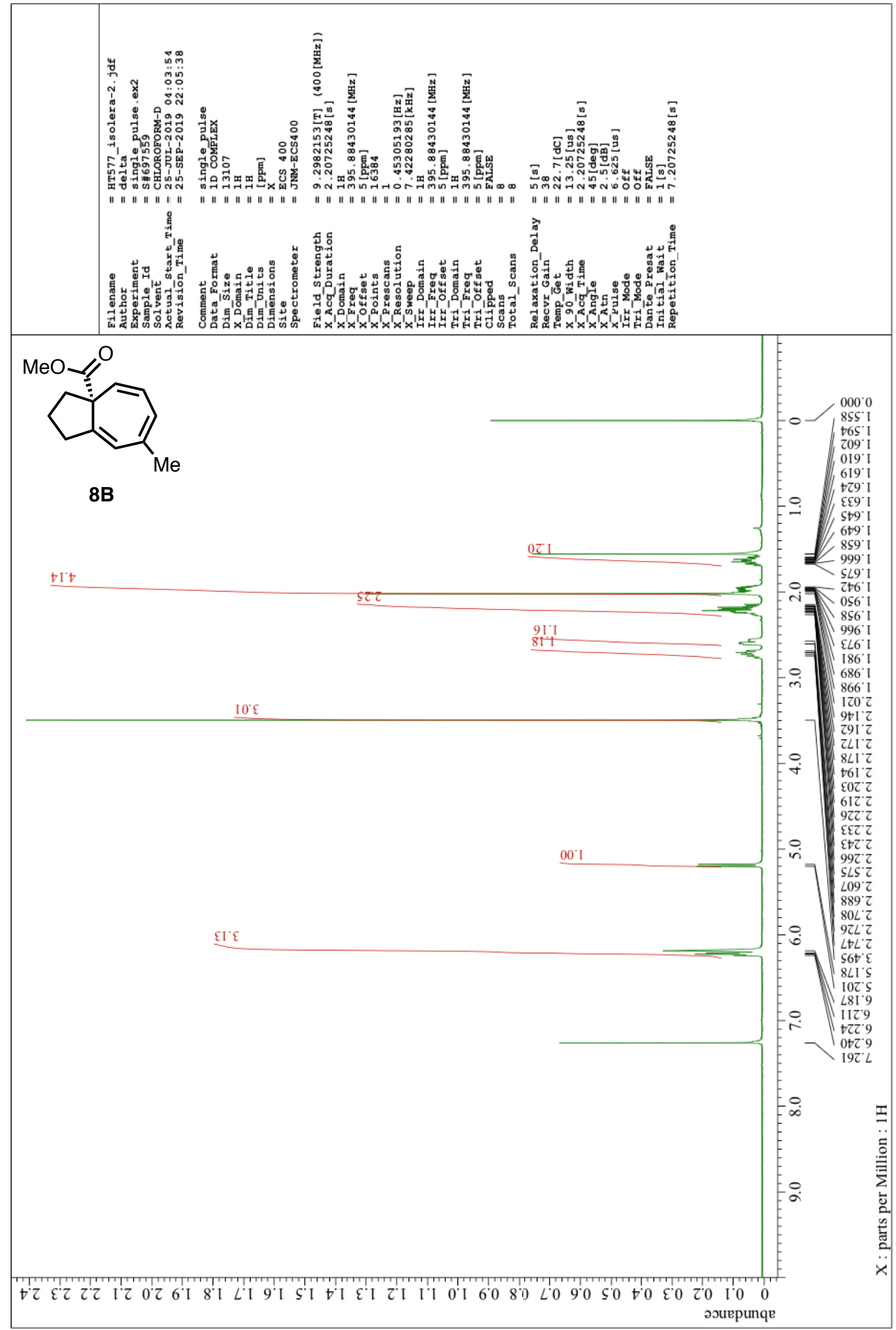


${ }^{13} \mathrm{C}$ NMR of 8 B $\left(101 \mathrm{MHz}, \mathrm{CDCl}_{3}\right)$

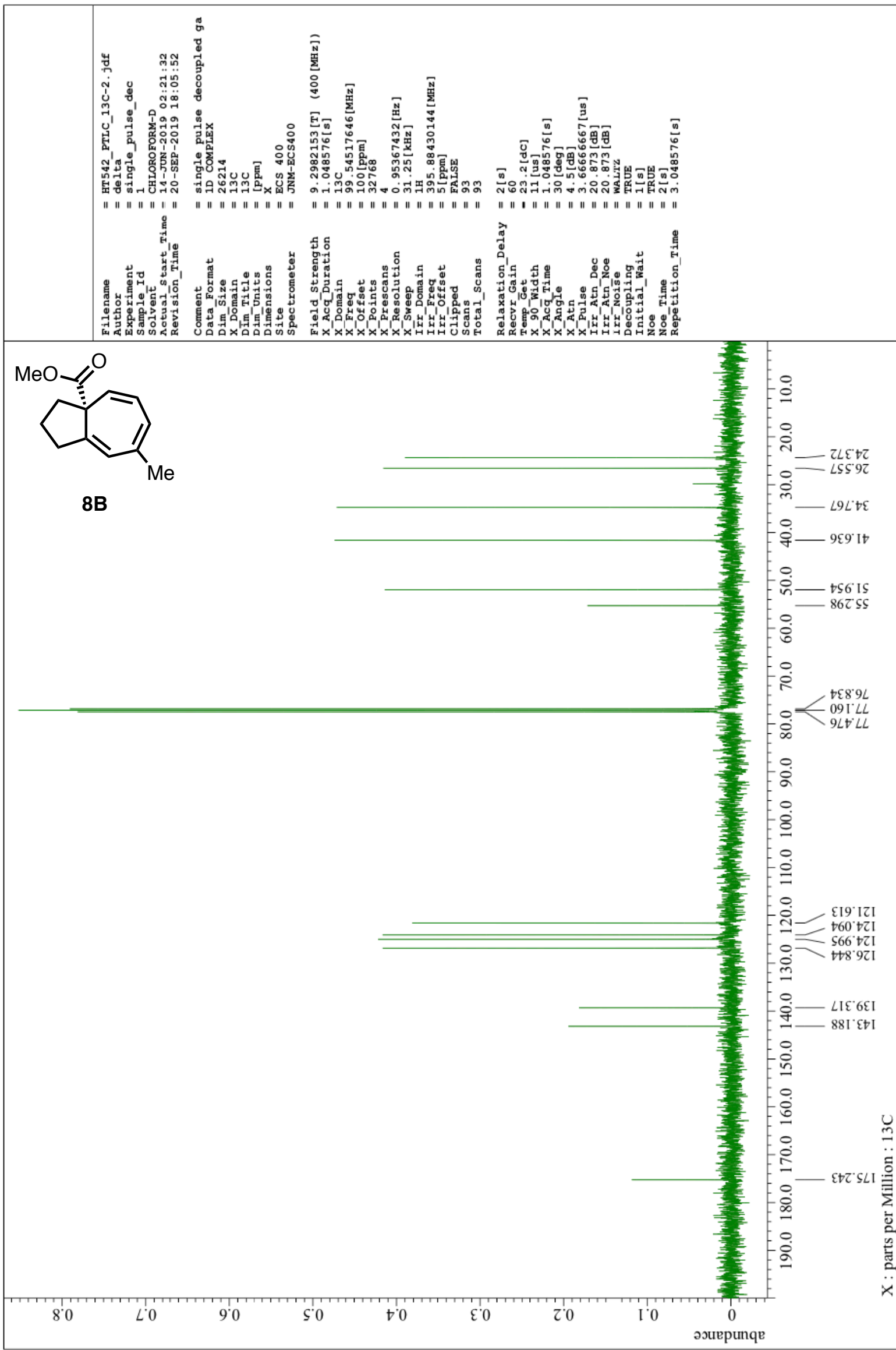


${ }^{1} \mathrm{H}$ NMR of $8 \mathrm{C}\left(400 \mathrm{MHz}, \mathrm{CDCl}_{3}\right)$

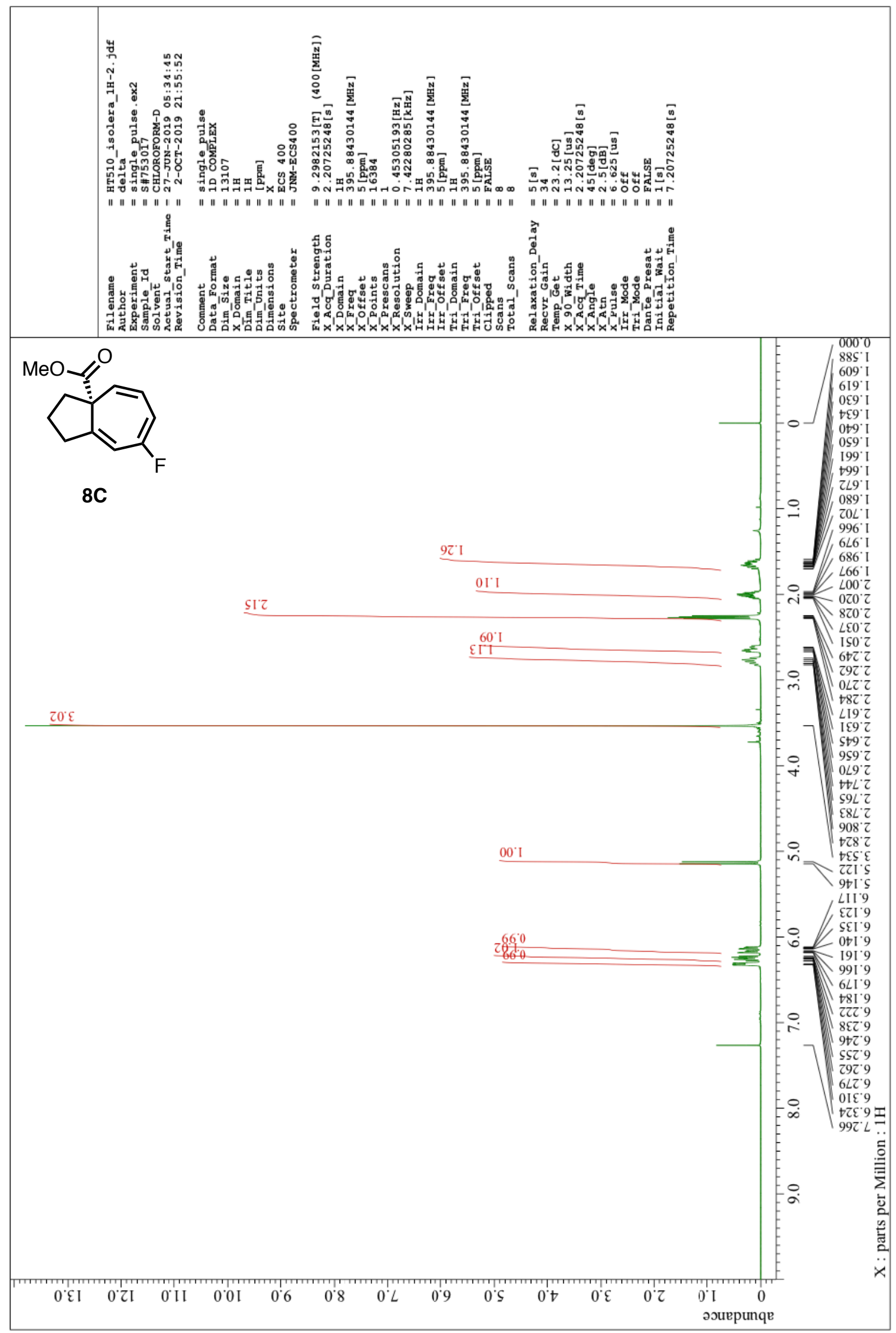


${ }^{13} \mathrm{C}$ NMR of $8 \mathrm{C}\left(101 \mathrm{MHz}, \mathrm{CDCl}_{3}\right)$

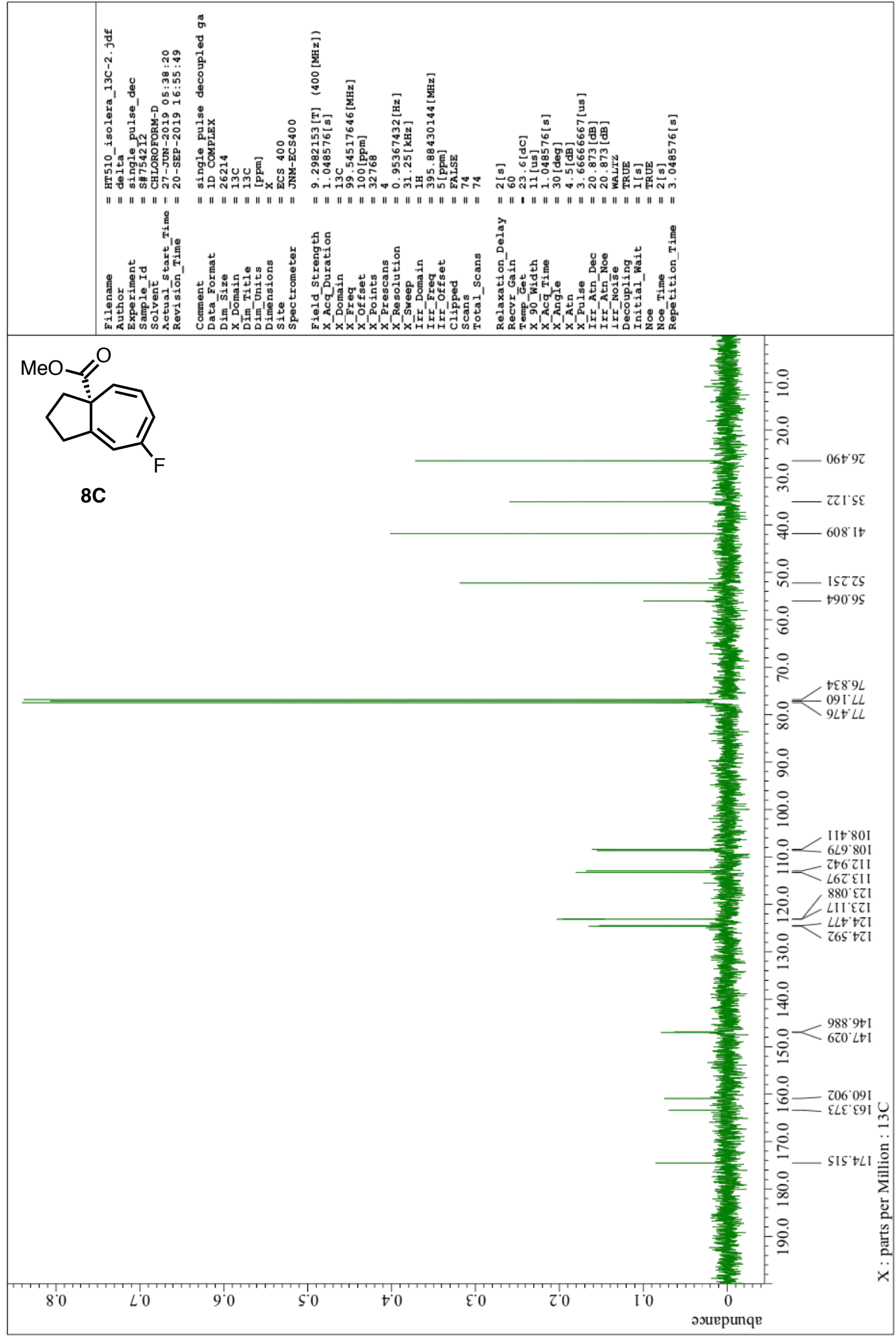


${ }^{1} \mathrm{H}$ NMR of $8 D\left(400 \mathrm{MHz}, \mathrm{CDCl}_{3}\right)$

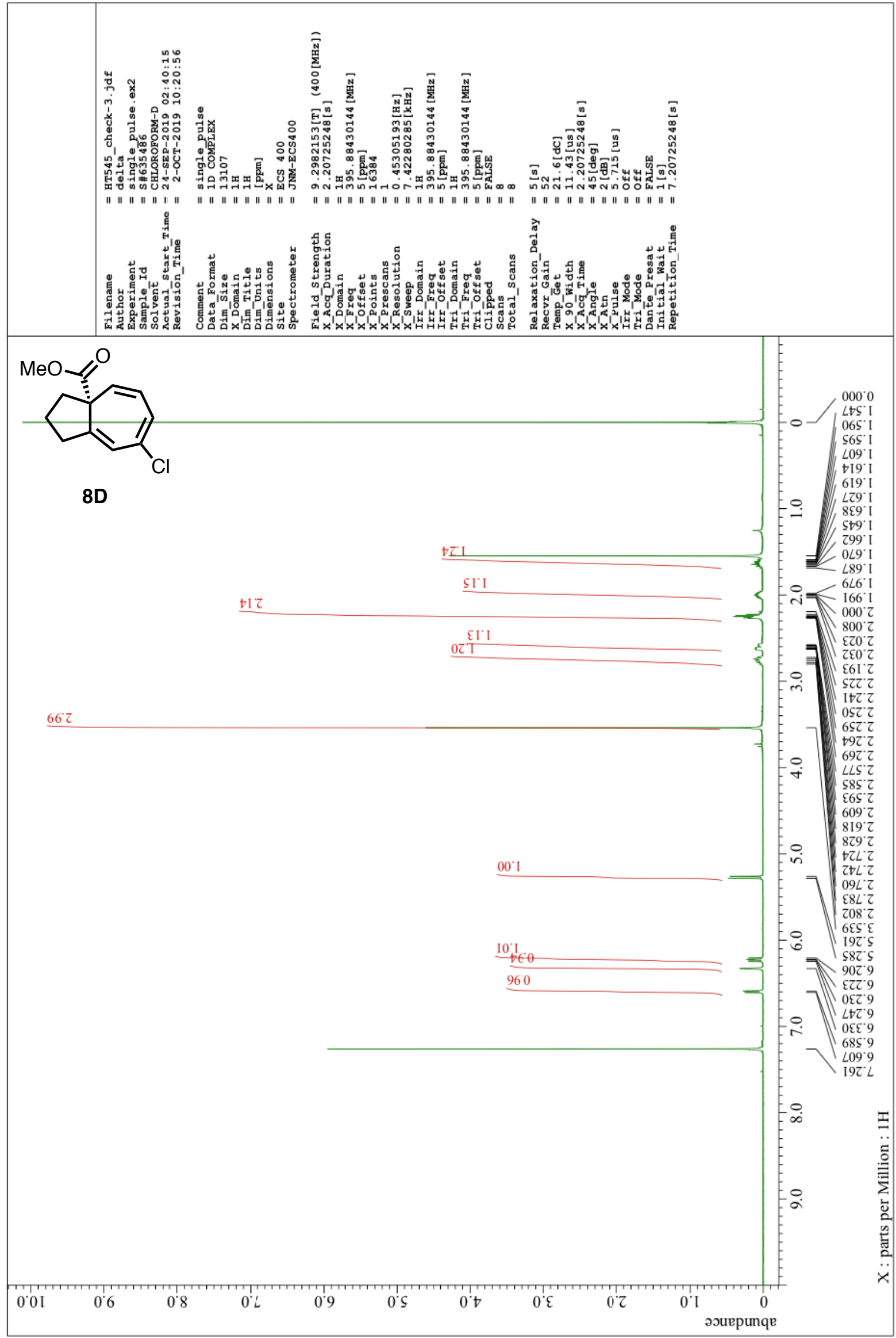


${ }^{13} \mathrm{C}$ NMR of $8 D\left(101 \mathrm{MHz}, \mathrm{CDCl}_{3}\right)$

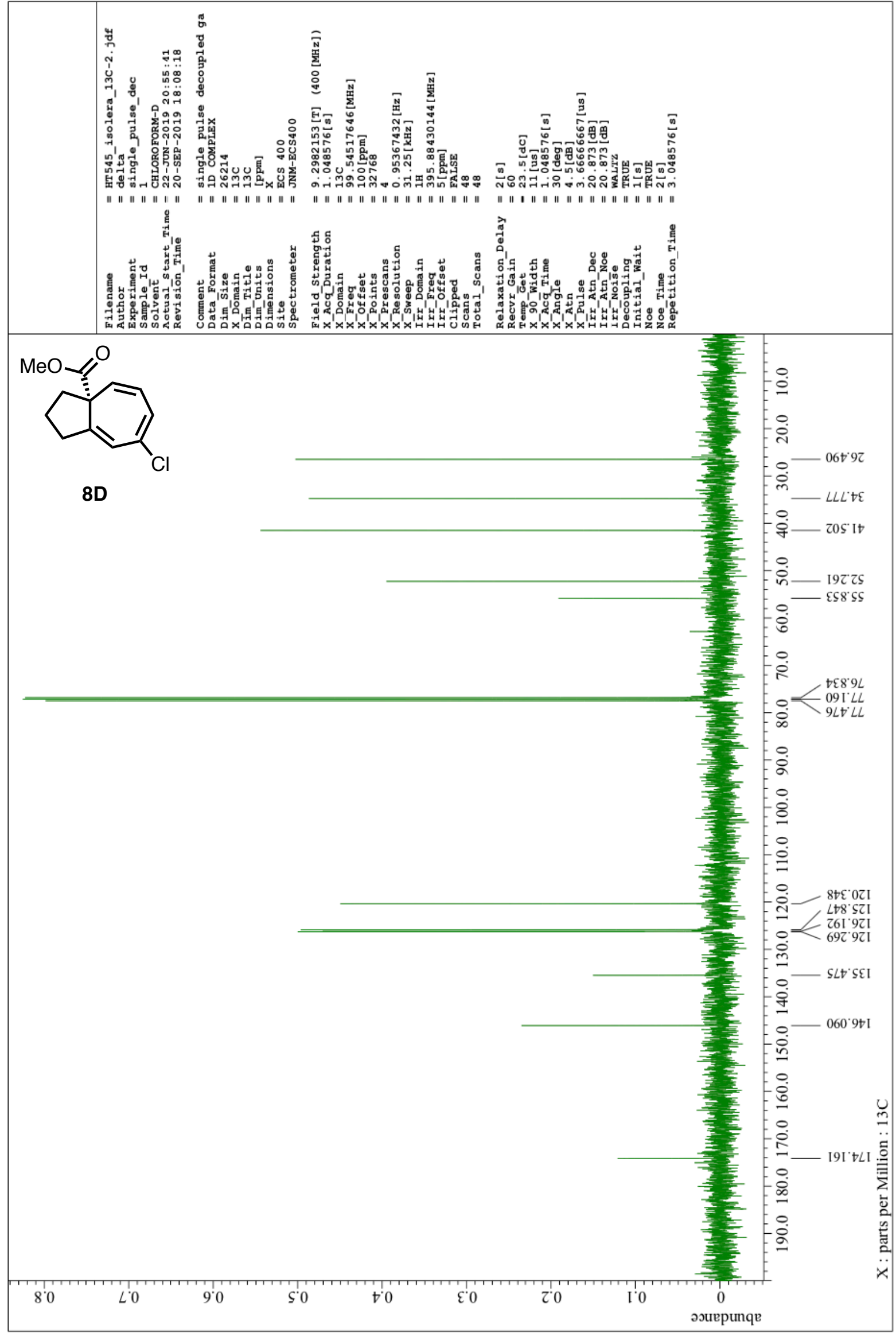


${ }^{1} \mathrm{H}$ NMR of $\mathbf{8 E}\left(400 \mathrm{MHz}, \mathrm{CDCl}_{3}\right)$

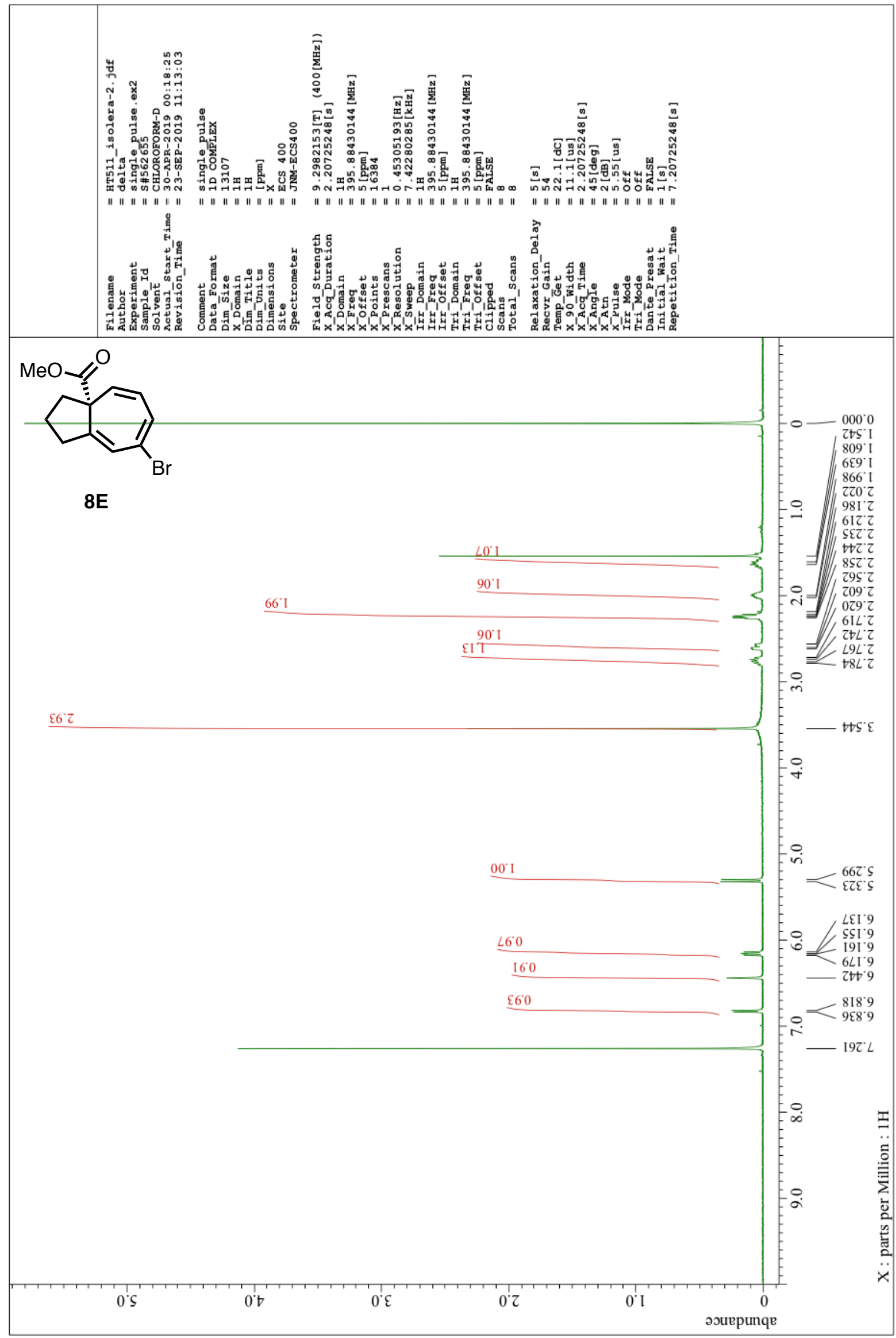


${ }^{13} \mathrm{C}$ NMR of $\mathbf{8 E}\left(101 \mathrm{MHz}, \mathrm{CDCl}_{3}\right)$

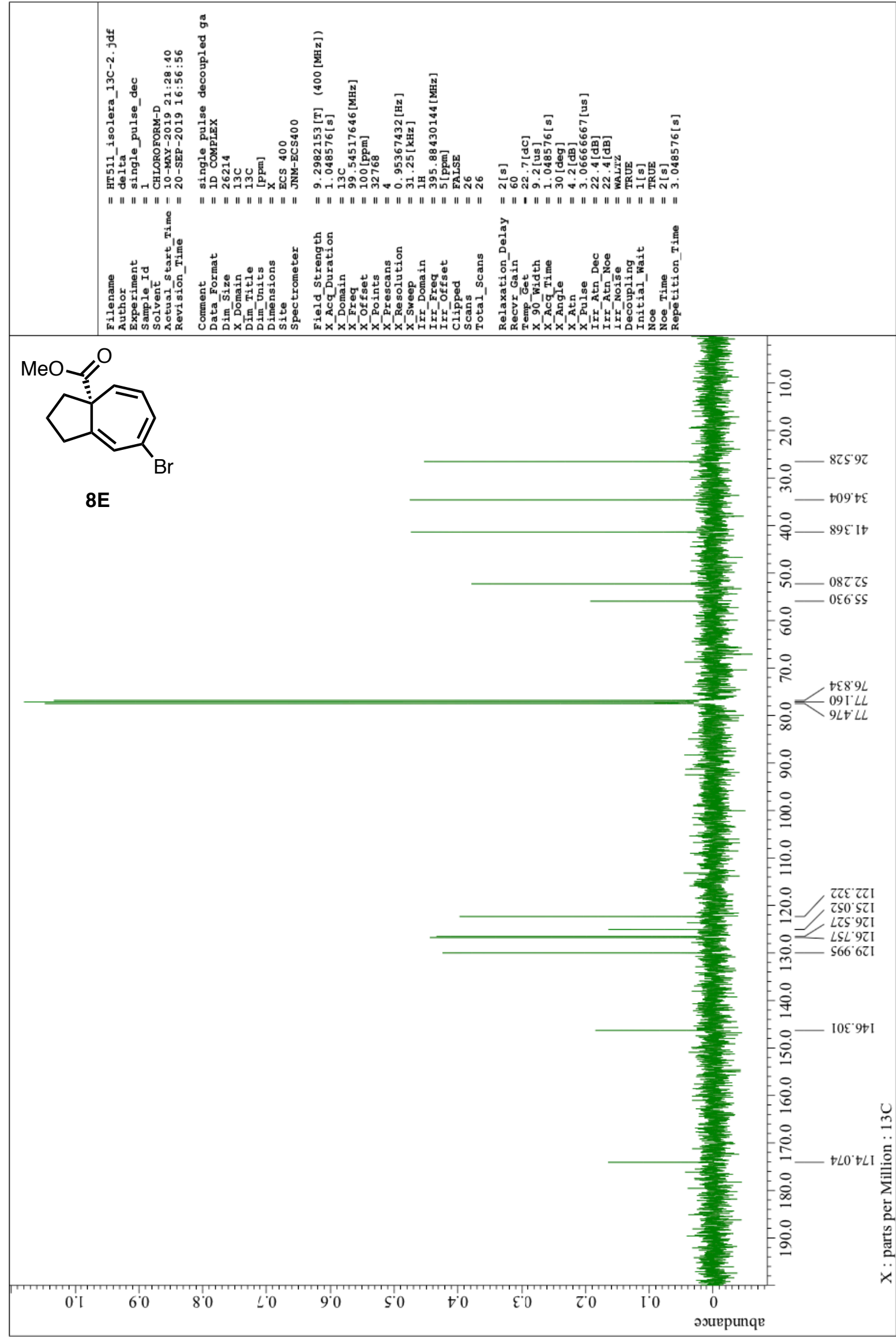


${ }^{1} \mathrm{H}$ NMR of $\mathbf{8 F}\left(400 \mathrm{MHz}, \mathrm{CDCl}_{3}\right)$

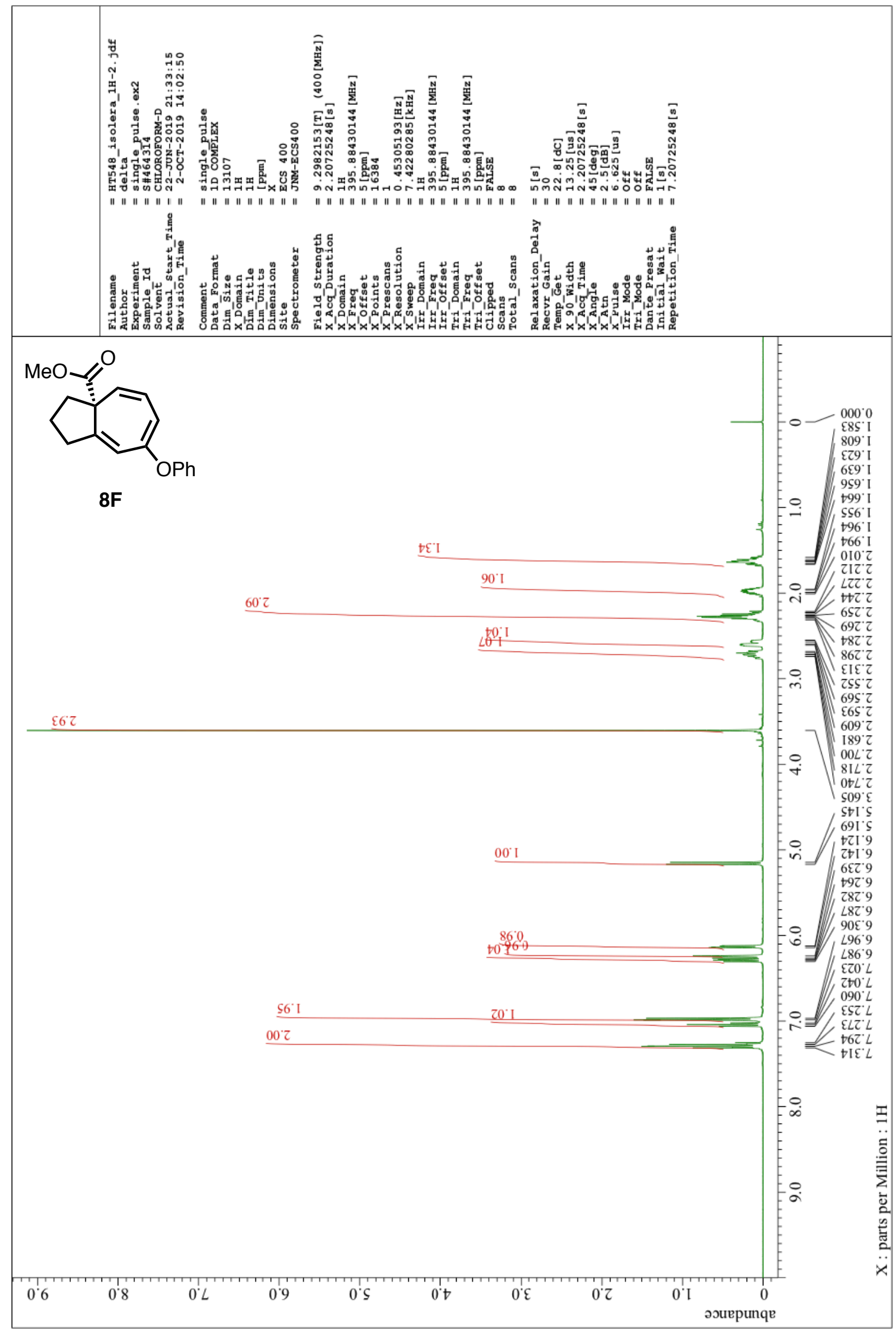


${ }^{13} \mathrm{C}$ NMR of $\mathbf{8 F}\left(101 \mathrm{MHz}, \mathrm{CDCl}_{3}\right)$

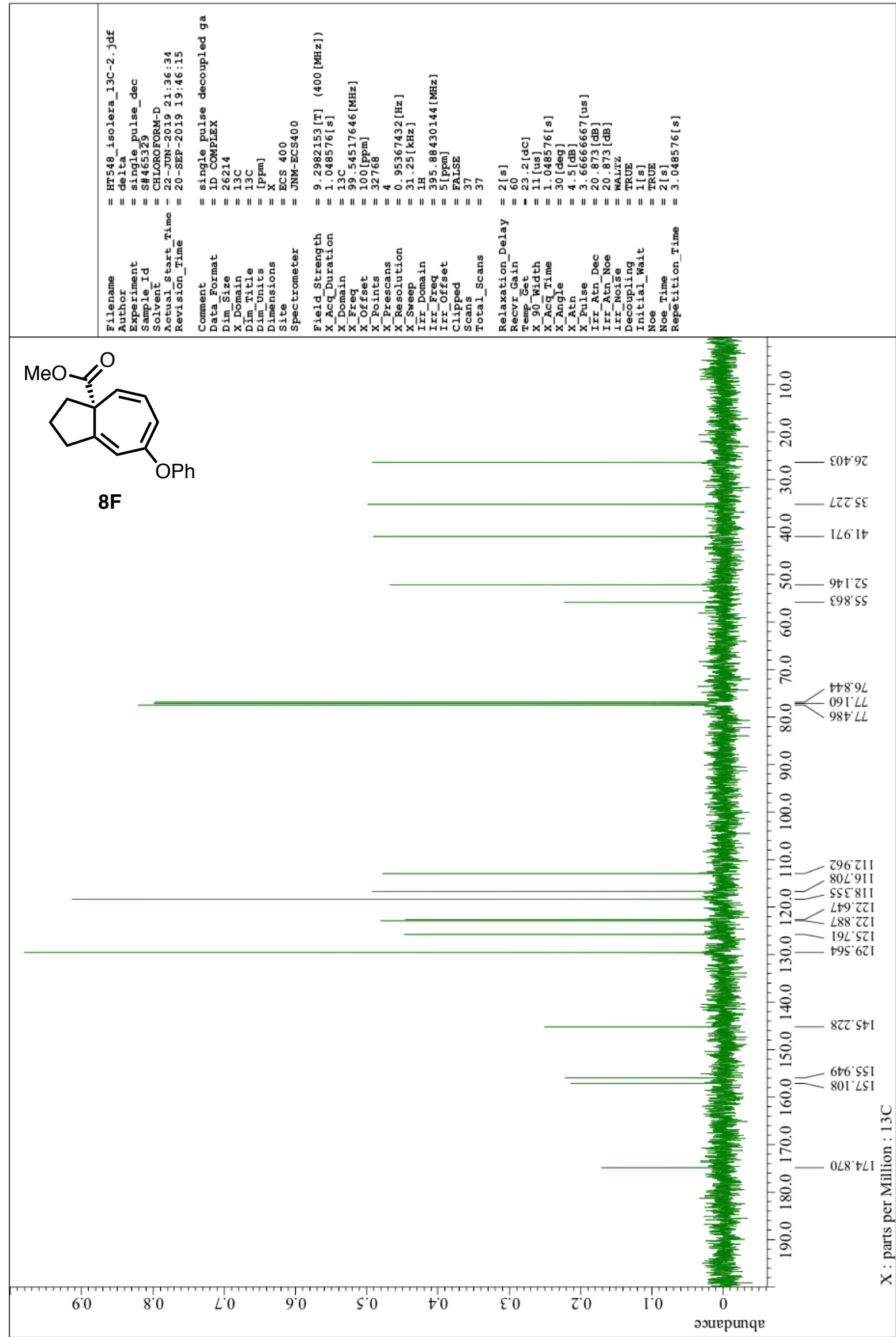


${ }^{1} \mathrm{H}$ NMR of $8 \mathbf{G}\left(400 \mathrm{MHz}, \mathrm{CDCl}_{3}\right)$

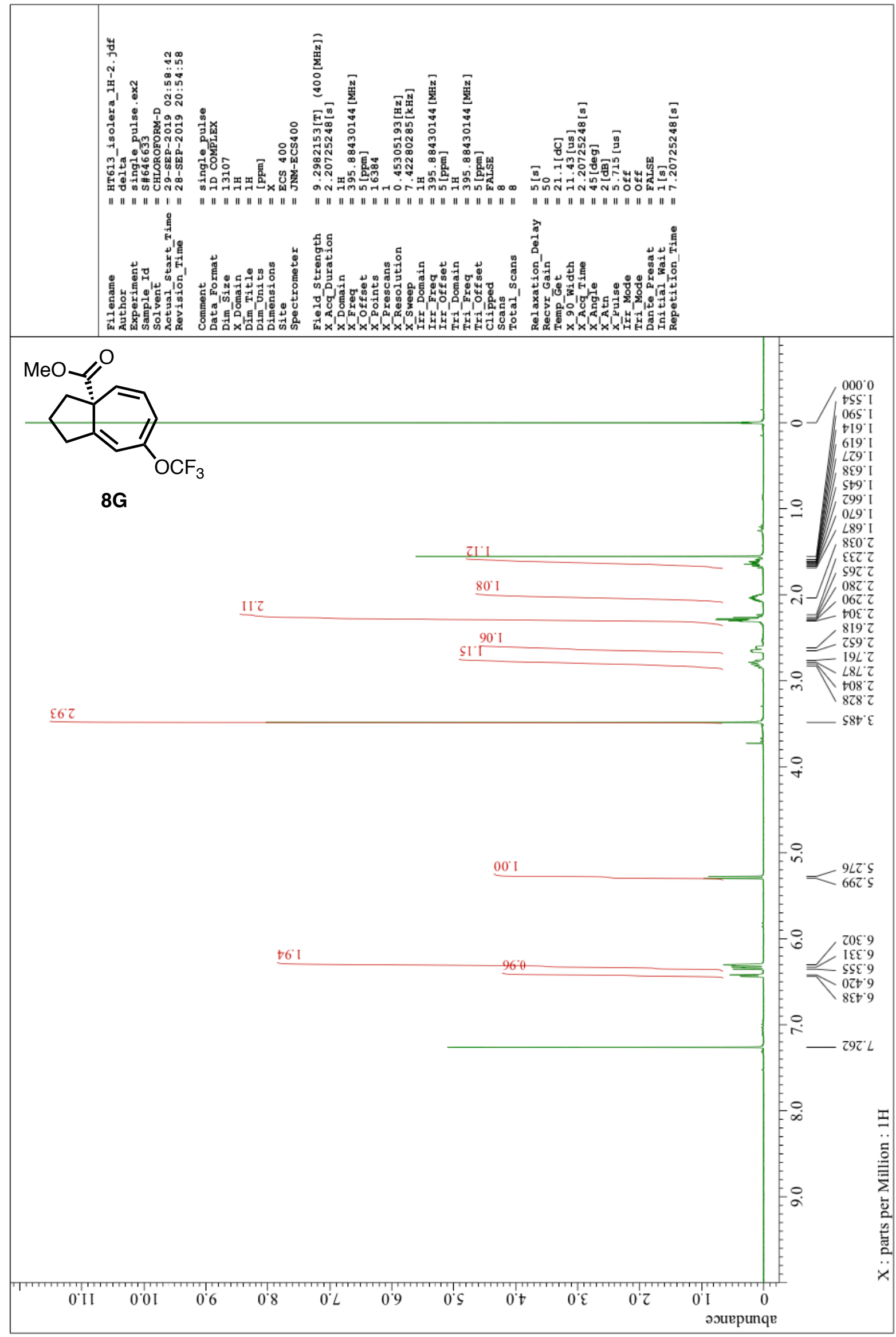


${ }^{13} \mathrm{C}$ NMR of $8 \mathbf{G}\left(101 \mathrm{MHz}, \mathrm{CDCl}_{3}\right)$

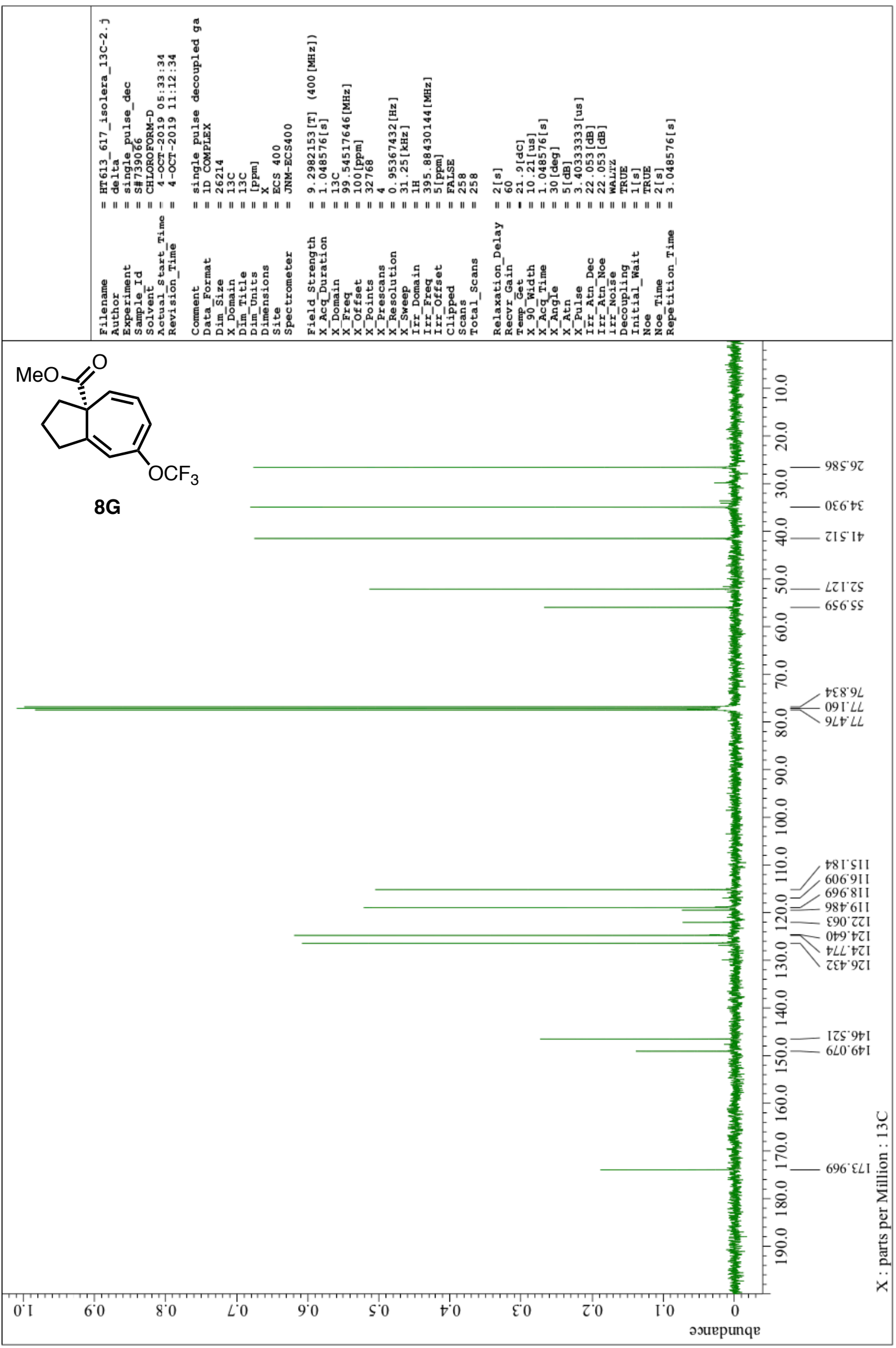


${ }^{1} \mathrm{H}$ NMR of $\mathbf{8 H}\left(400 \mathrm{MHz}, \mathrm{CDCl}_{3}\right)$

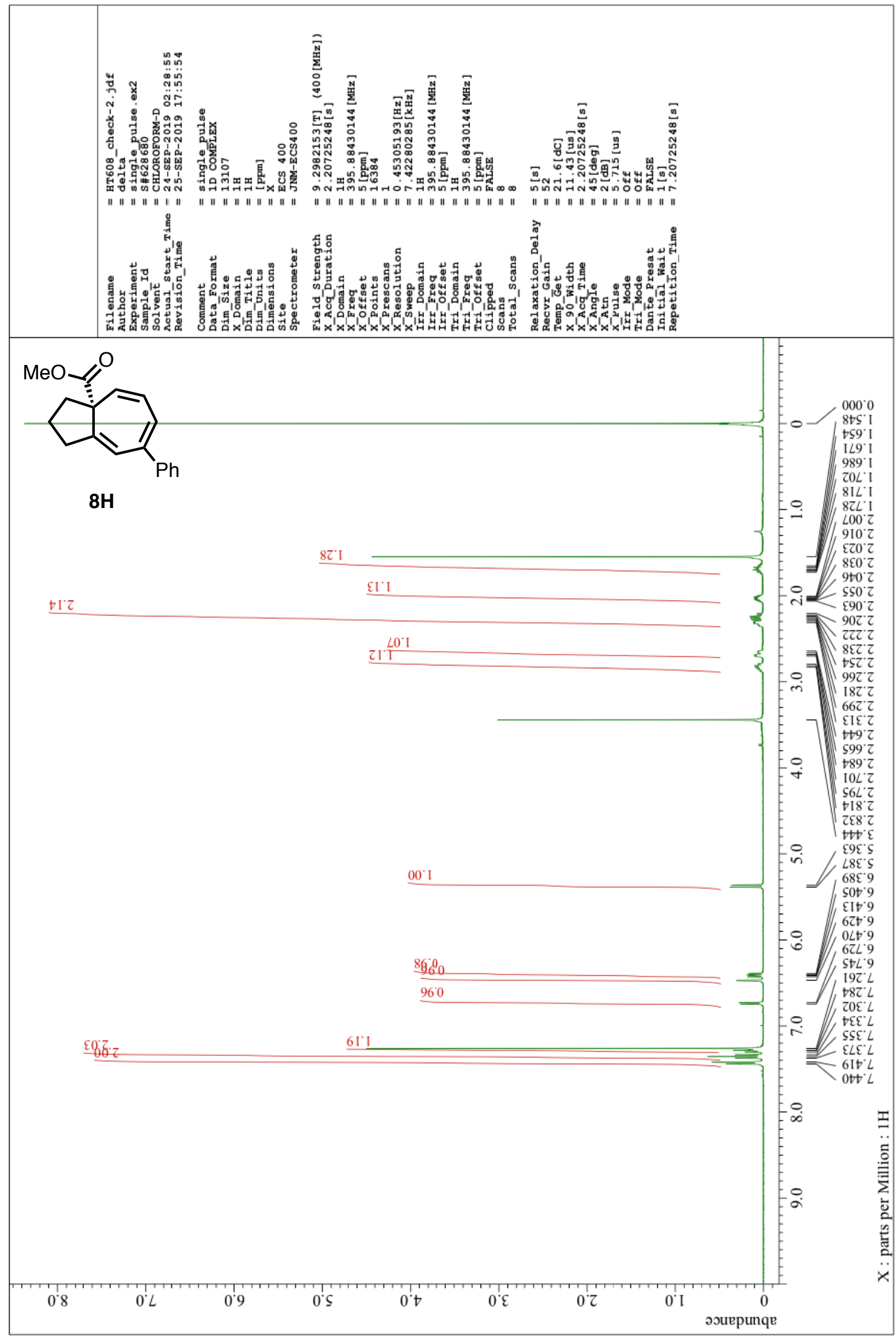


${ }^{13} \mathrm{C}$ NMR of $\mathbf{8 H}\left(101 \mathrm{MHz}, \mathrm{CDCl}_{3}\right)$

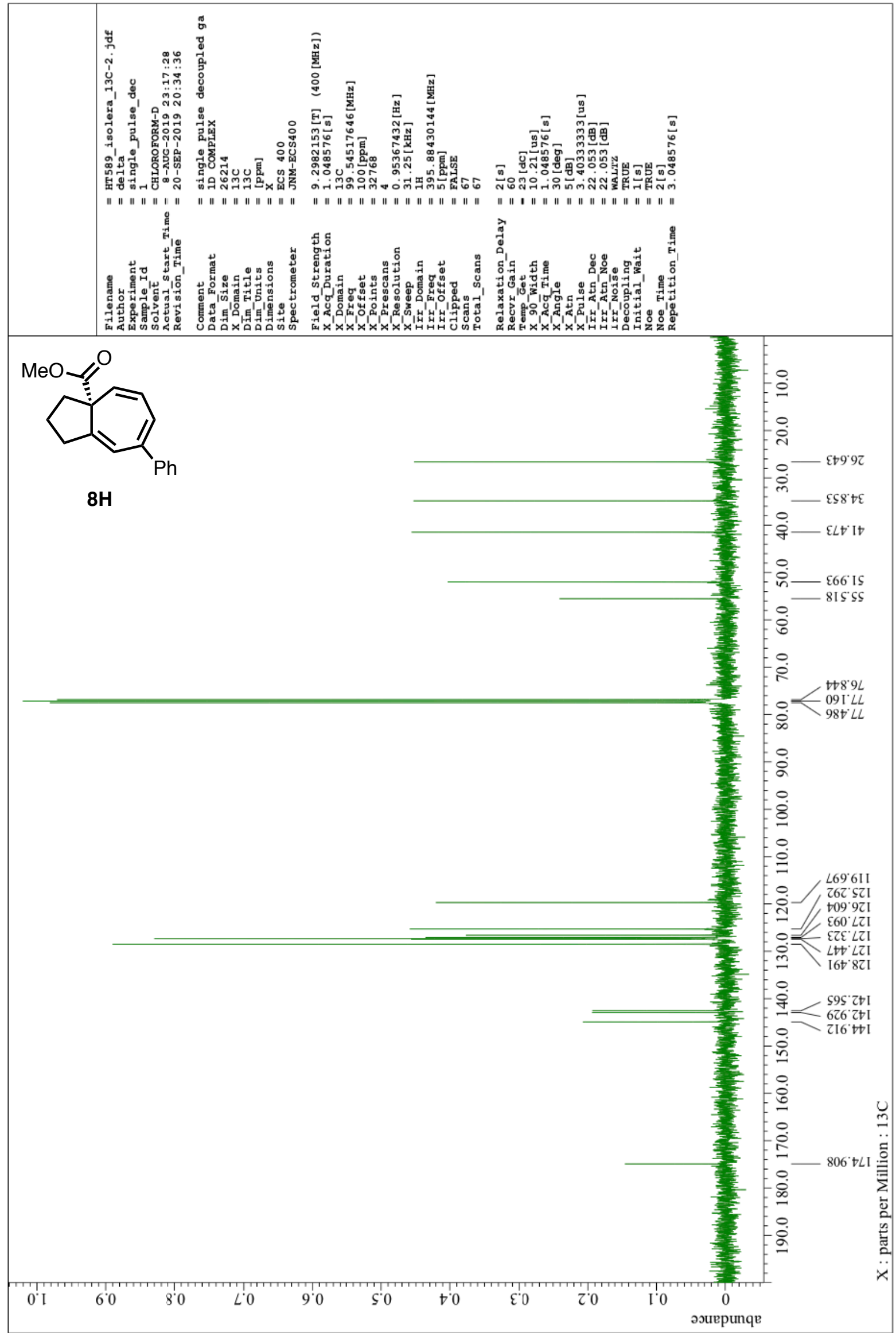


${ }^{1} \mathrm{H}$ NMR of $8 \mathbf{I}\left(400 \mathrm{MHz}, \mathrm{CDCl}_{3}\right)$

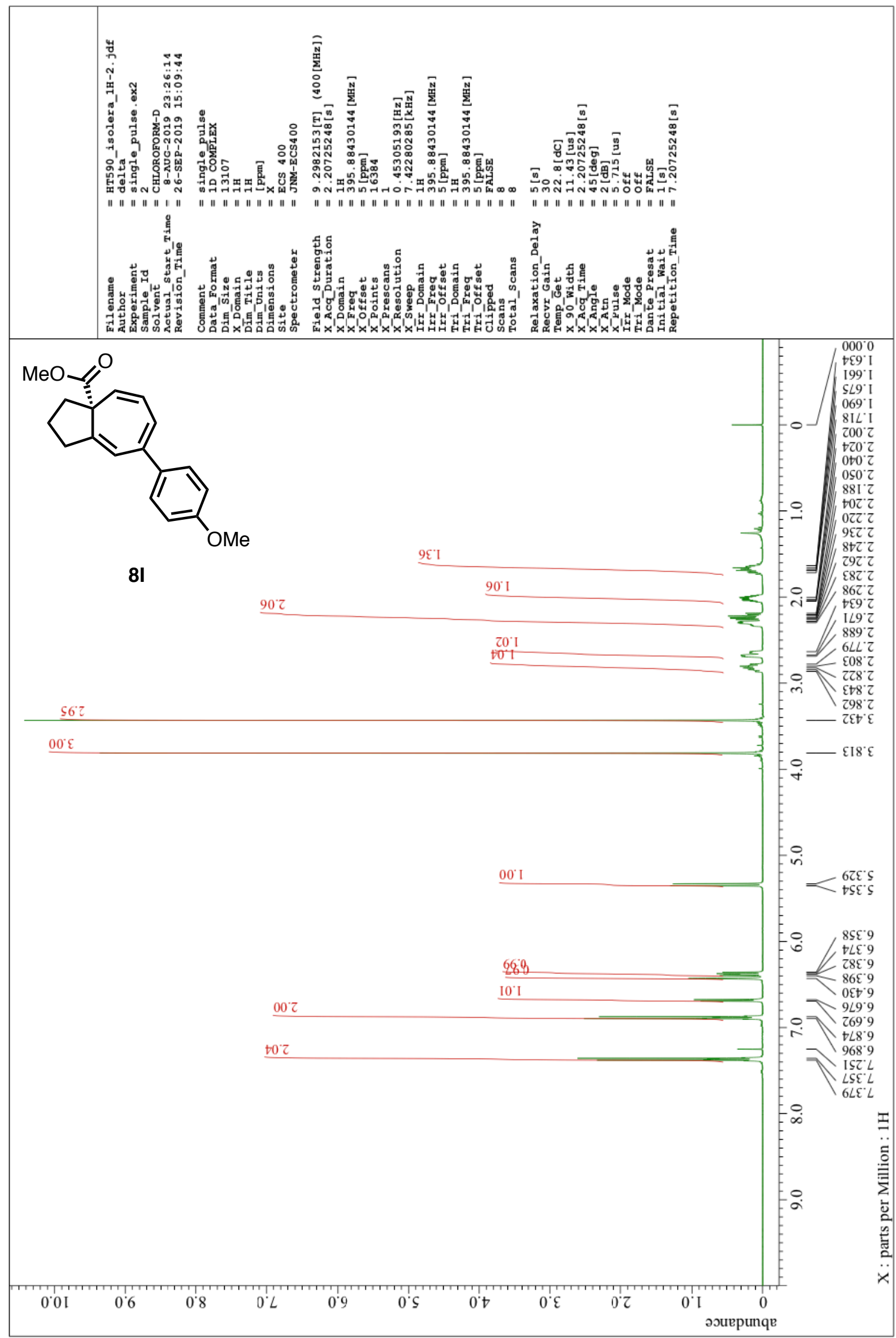


${ }^{13} \mathrm{C}$ NMR of $8 \mathbf{I}\left(101 \mathrm{MHz}, \mathrm{CDCl}_{3}\right)$

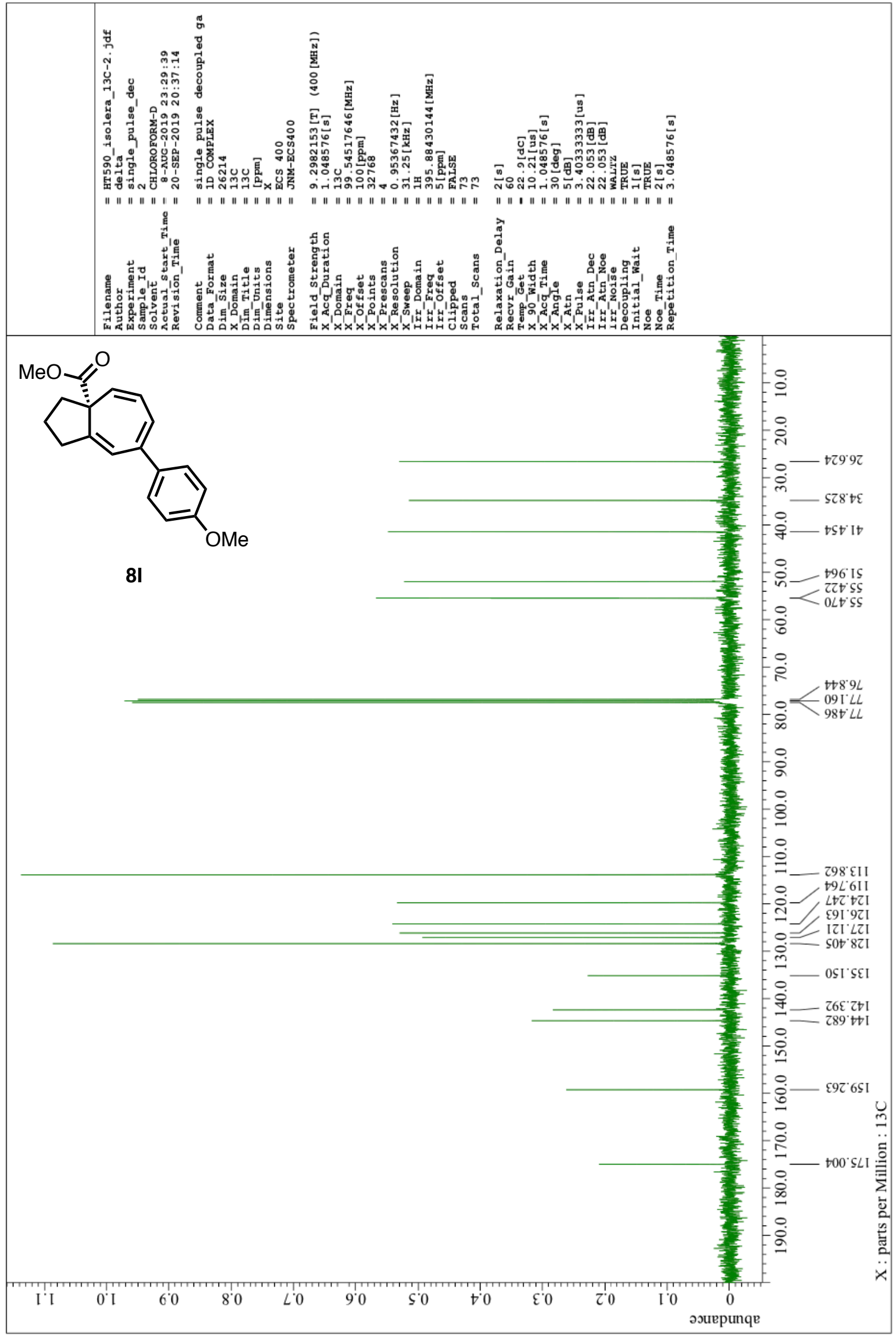


${ }^{1} \mathrm{H}$ NMR of $8 \mathbf{J}\left(400 \mathrm{MHz}, \mathrm{CDCl}_{3}\right)$

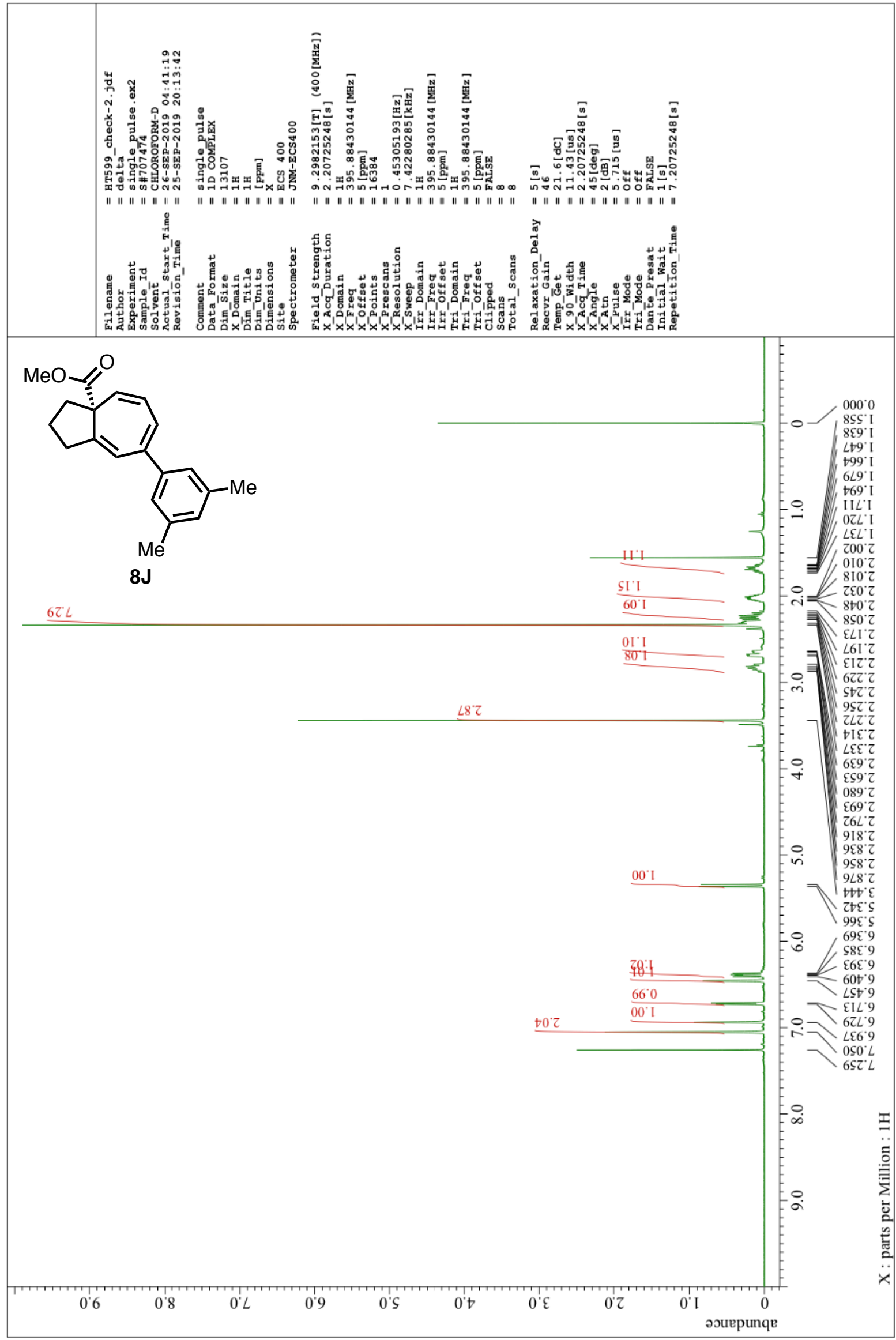


${ }^{13} \mathrm{C}$ NMR of $\mathbf{8 J}\left(101 \mathrm{MHz}, \mathrm{CDCl}_{3}\right)$

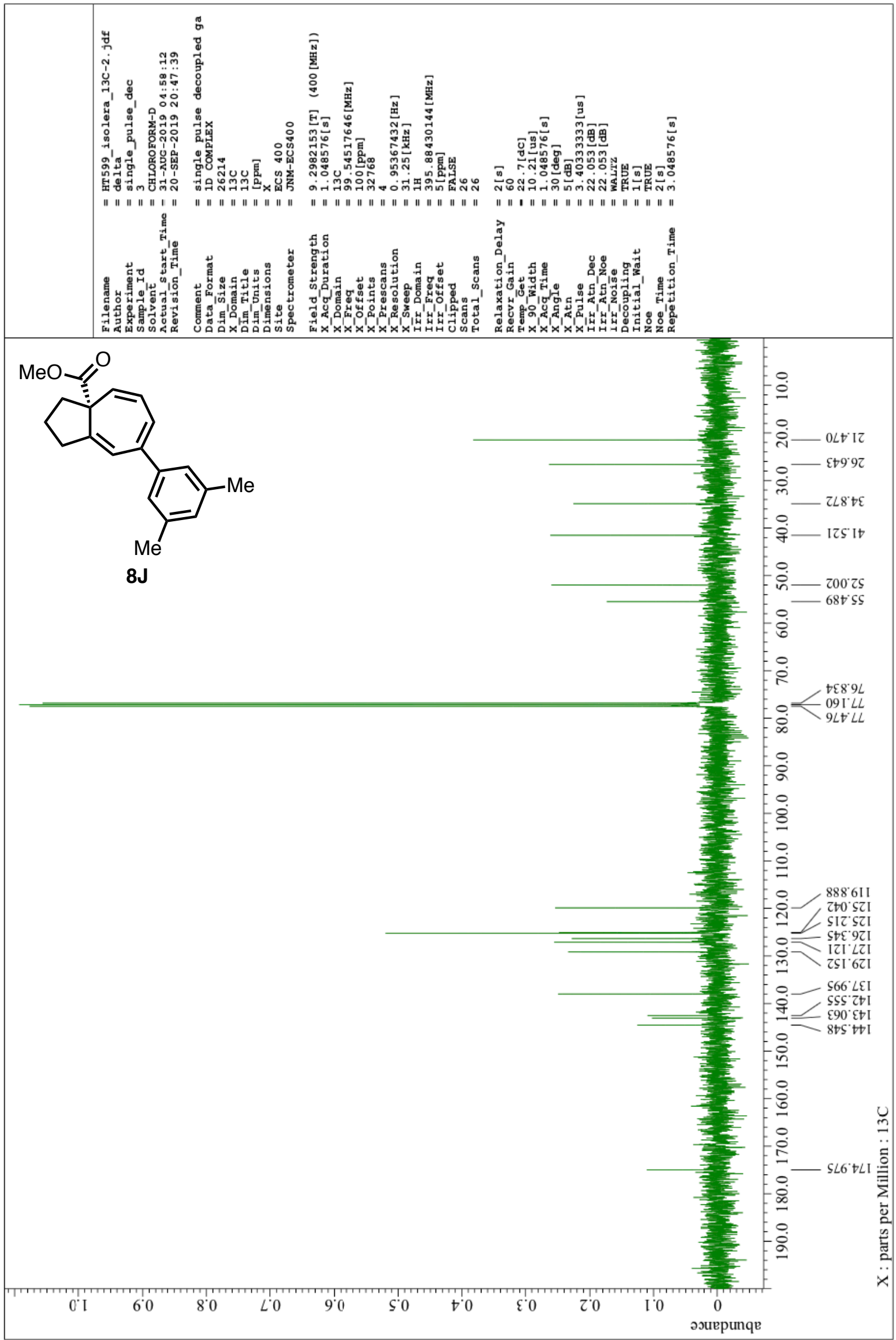


${ }^{1} \mathrm{H}$ NMR of $\mathbf{8 K}\left(400 \mathrm{MHz}, \mathrm{CDCl}_{3}\right)$

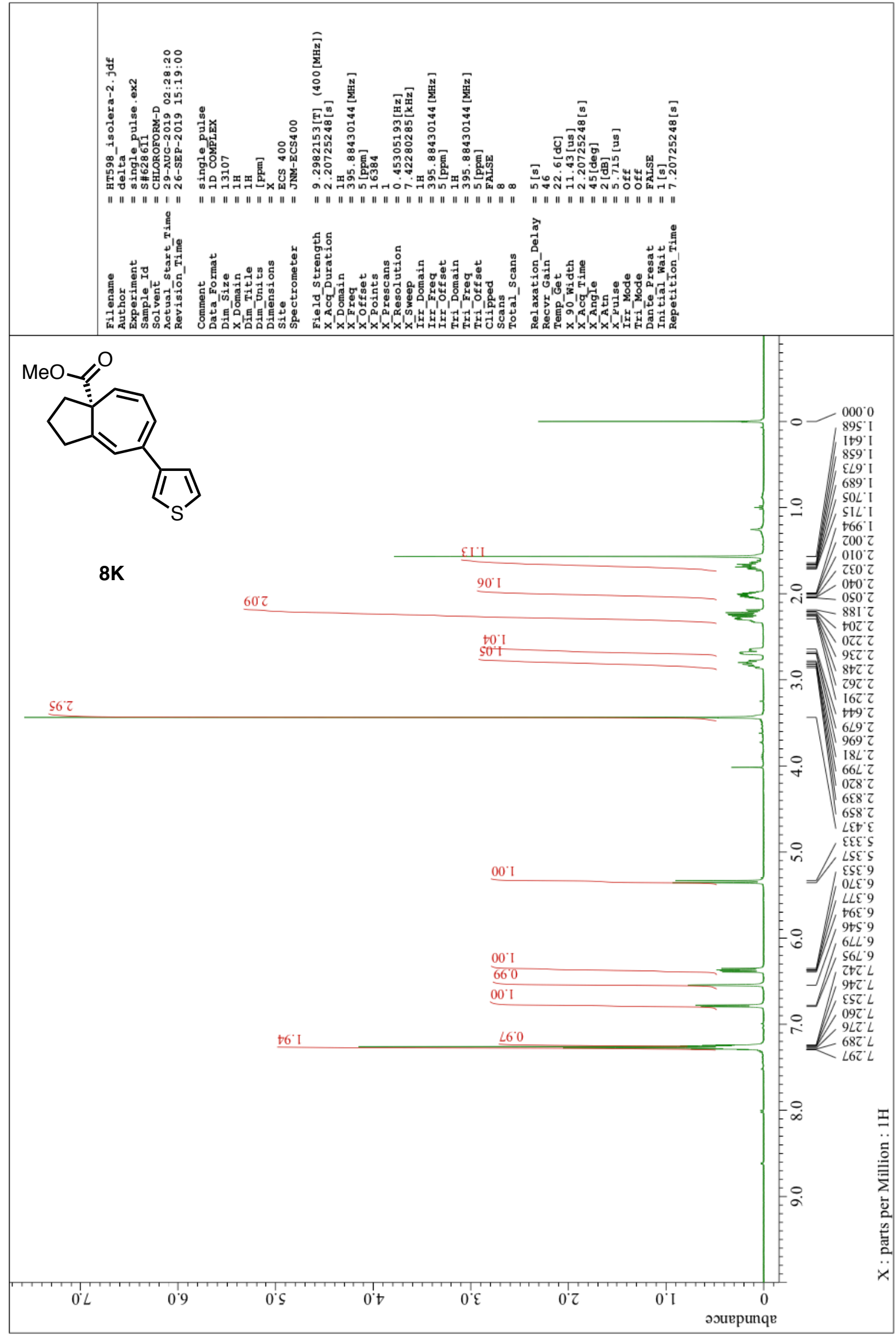


${ }^{13} \mathrm{C}$ NMR of $\mathbf{8 K}\left(101 \mathrm{MHz}, \mathrm{CDCl}_{3}\right)$

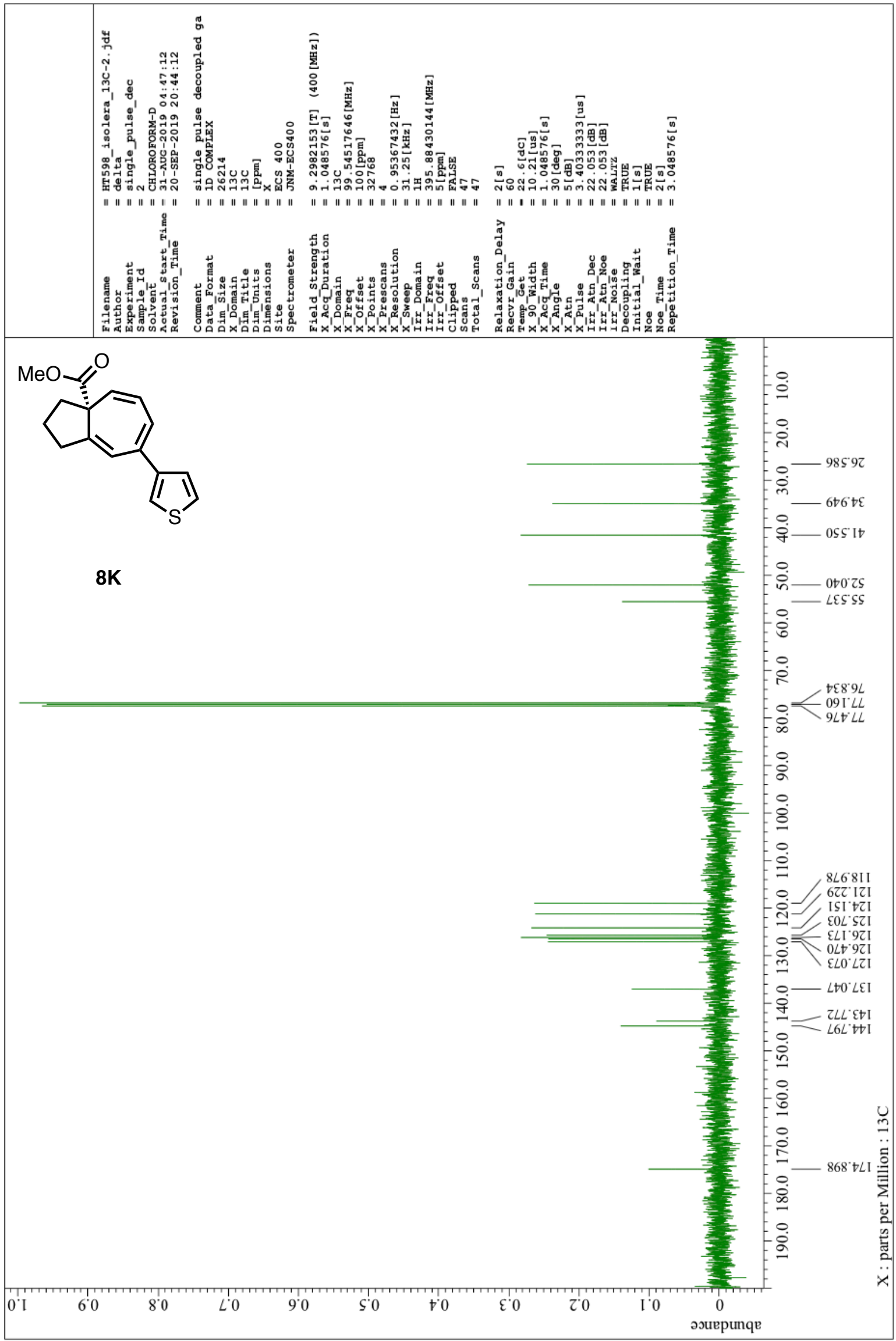




\section{HPLC spectra}

\section{Chromatogram Report}

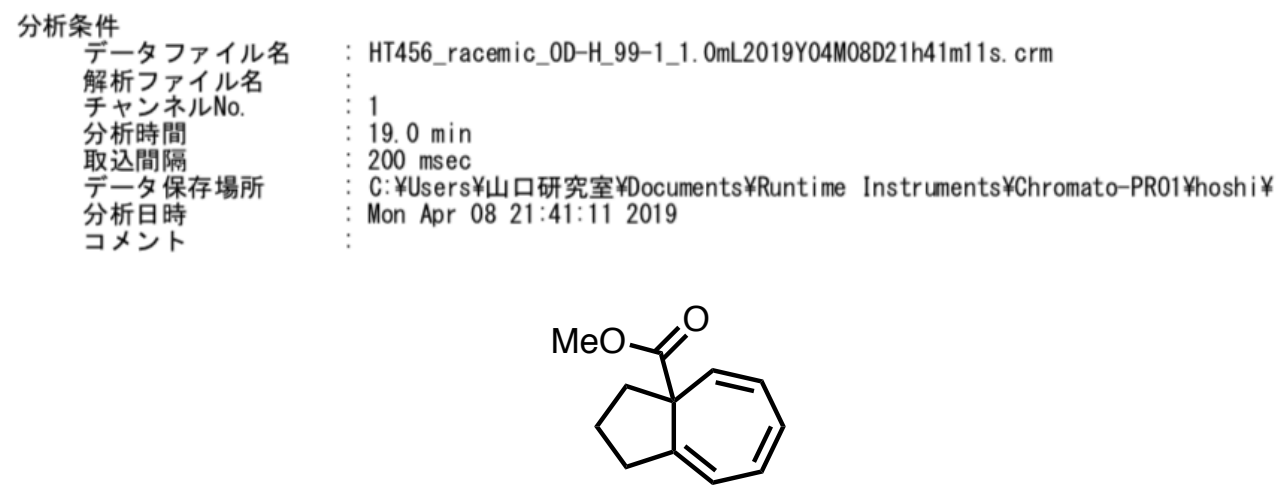

racemic $2 \mathrm{~A}$

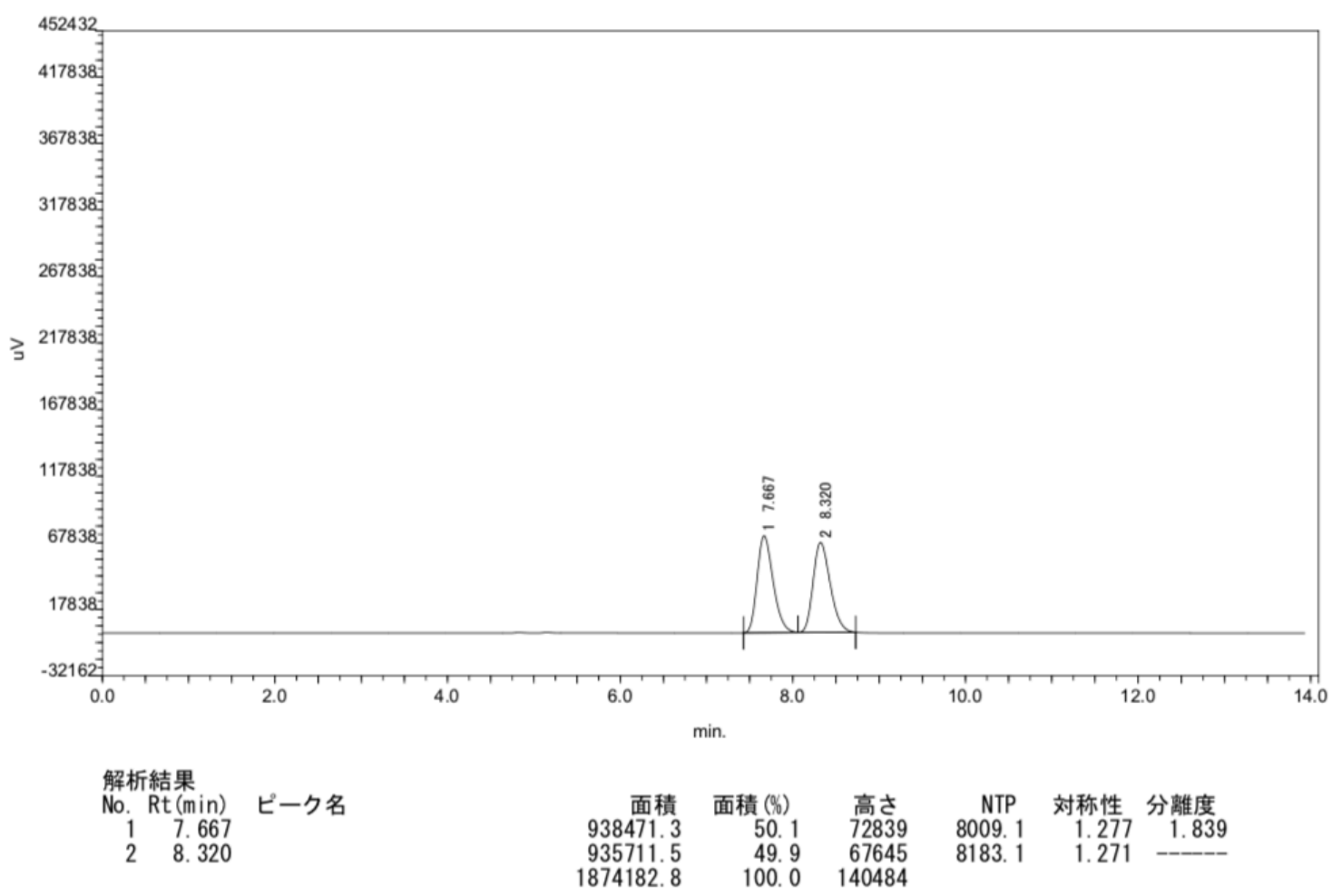




\section{Chromatogram Report}

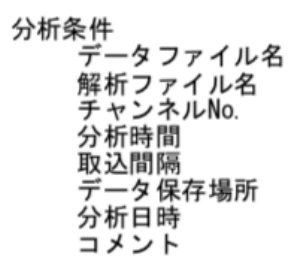

: HT489_S-TCPTAD_0D-H_99-1_1. OmL_check2019Y04M11D14h52m08s.crm

14. $0 \mathrm{~min}$

$200 \mathrm{msec}$

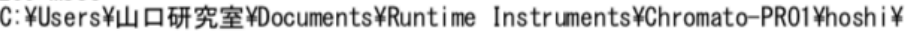

Thu Apr 11 14:52:08 2019

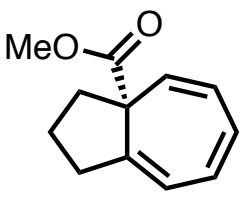

$2 A$

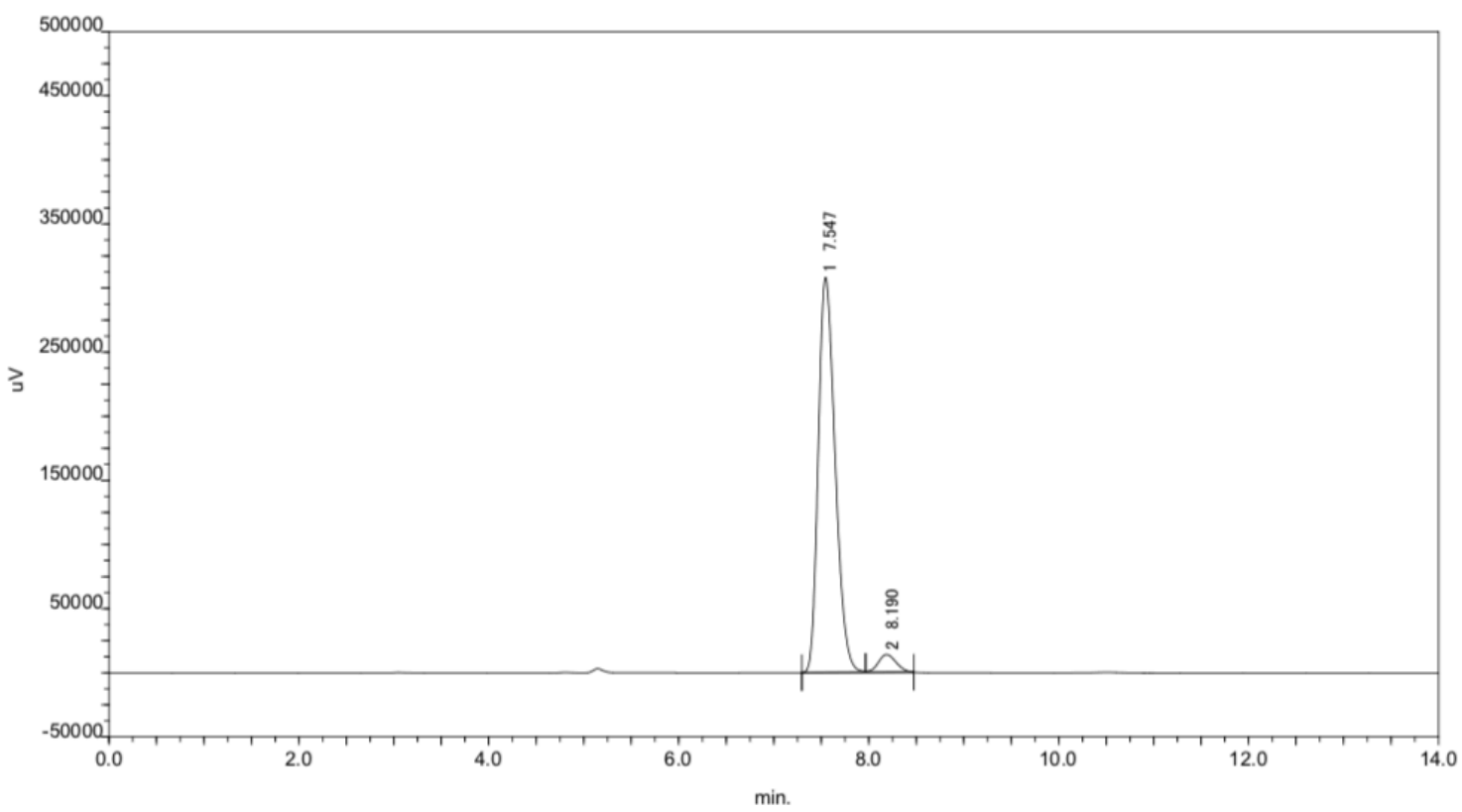

\begin{tabular}{rrr} 
面積 & \multicolumn{1}{c}{ 面積 $(\%)$} & \multicolumn{1}{c}{ 高さ } \\
3800424.2 & 95.4 & 308066 \\
182317.1 & 4.6 & 13878
\end{tabular}

$\begin{array}{rrr}182317.1 & 4.6 & 13878 \\ 3982741.3 & 100.0 & 321944\end{array}$
NTP 対称性 分離度

$\begin{array}{lll}8464.8 & 1.271 & 1.899\end{array}$
17.547 


\section{Chromatogram Report}

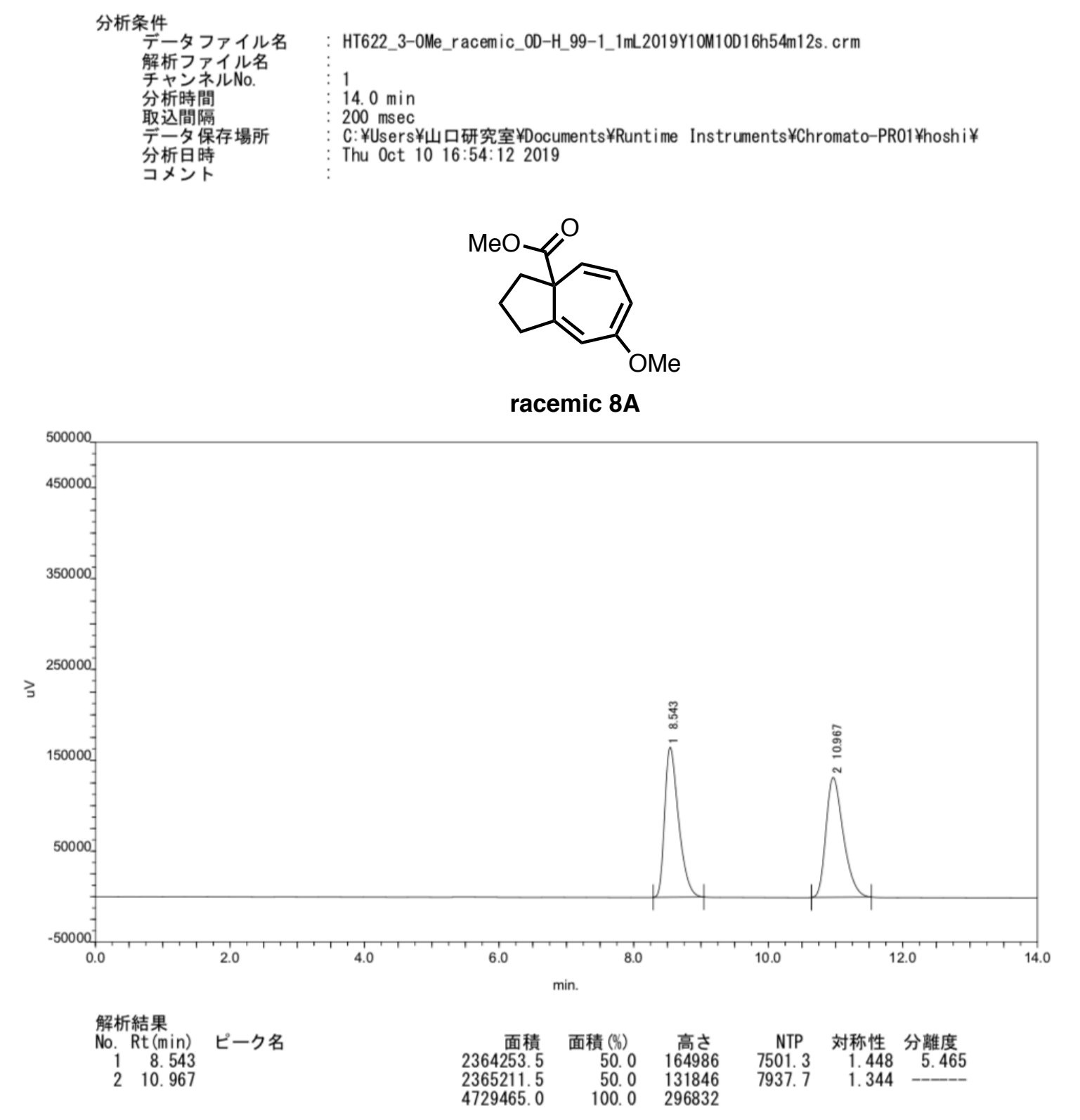




\section{Chromatogram Report}

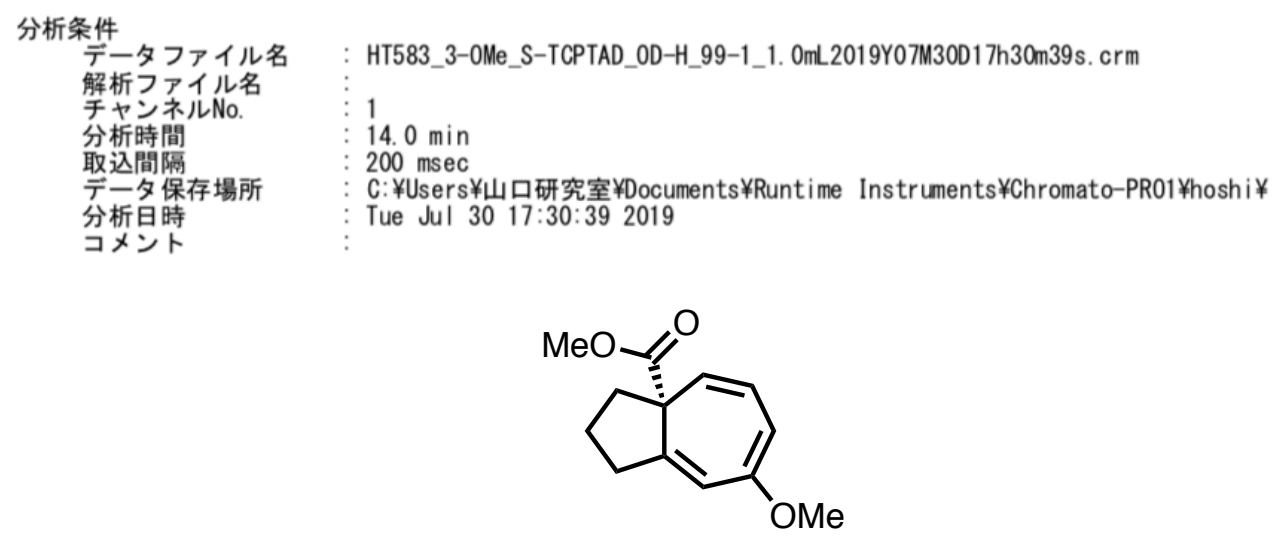

8A

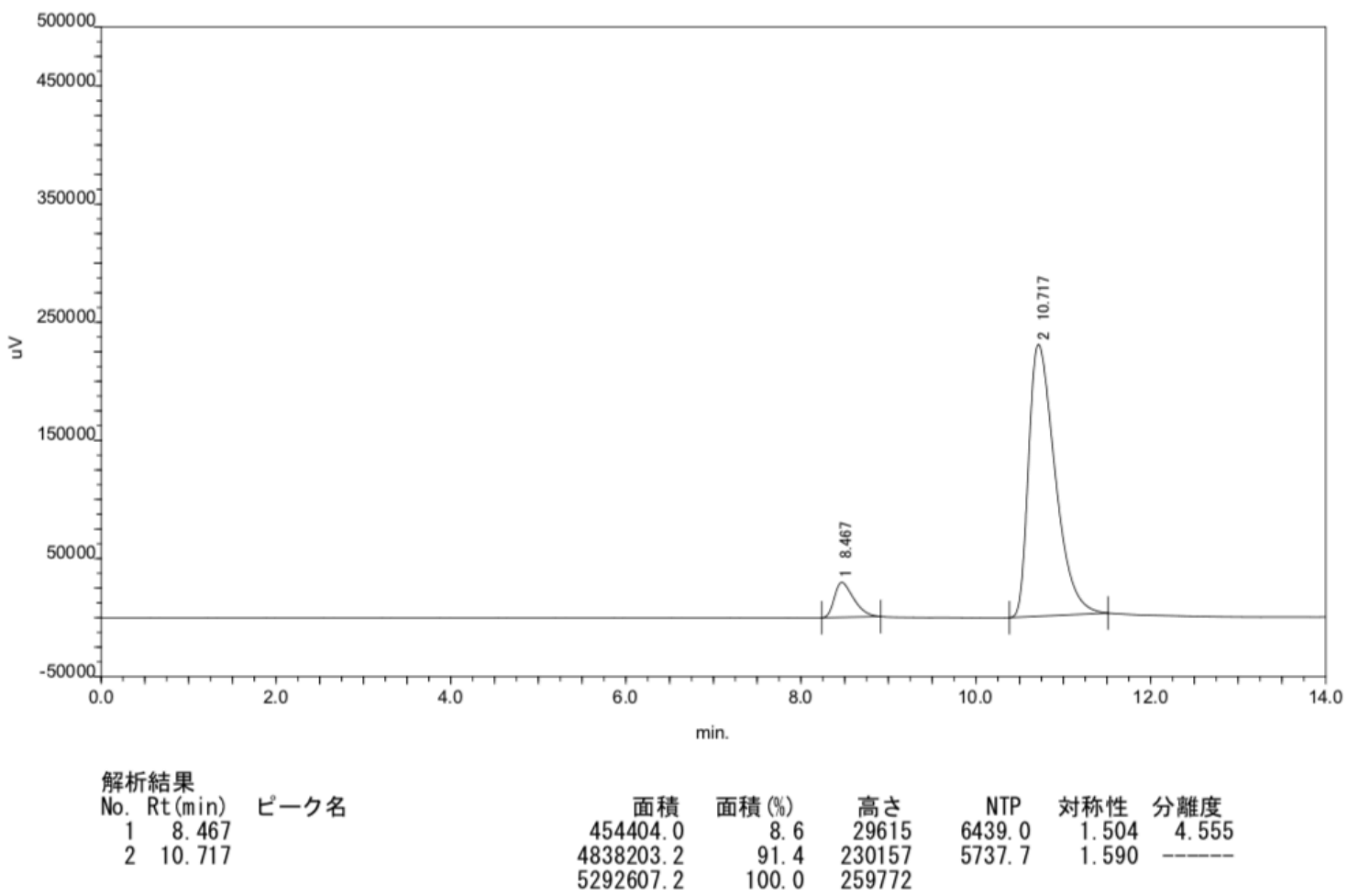




\section{Chromatogram Report}
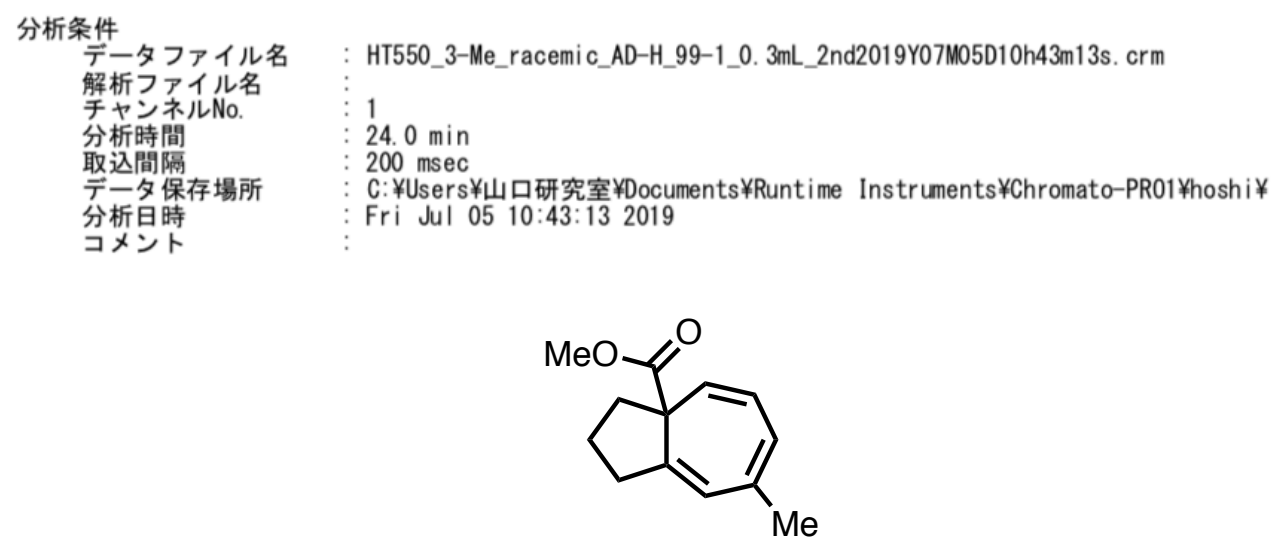

racemic $8 B$

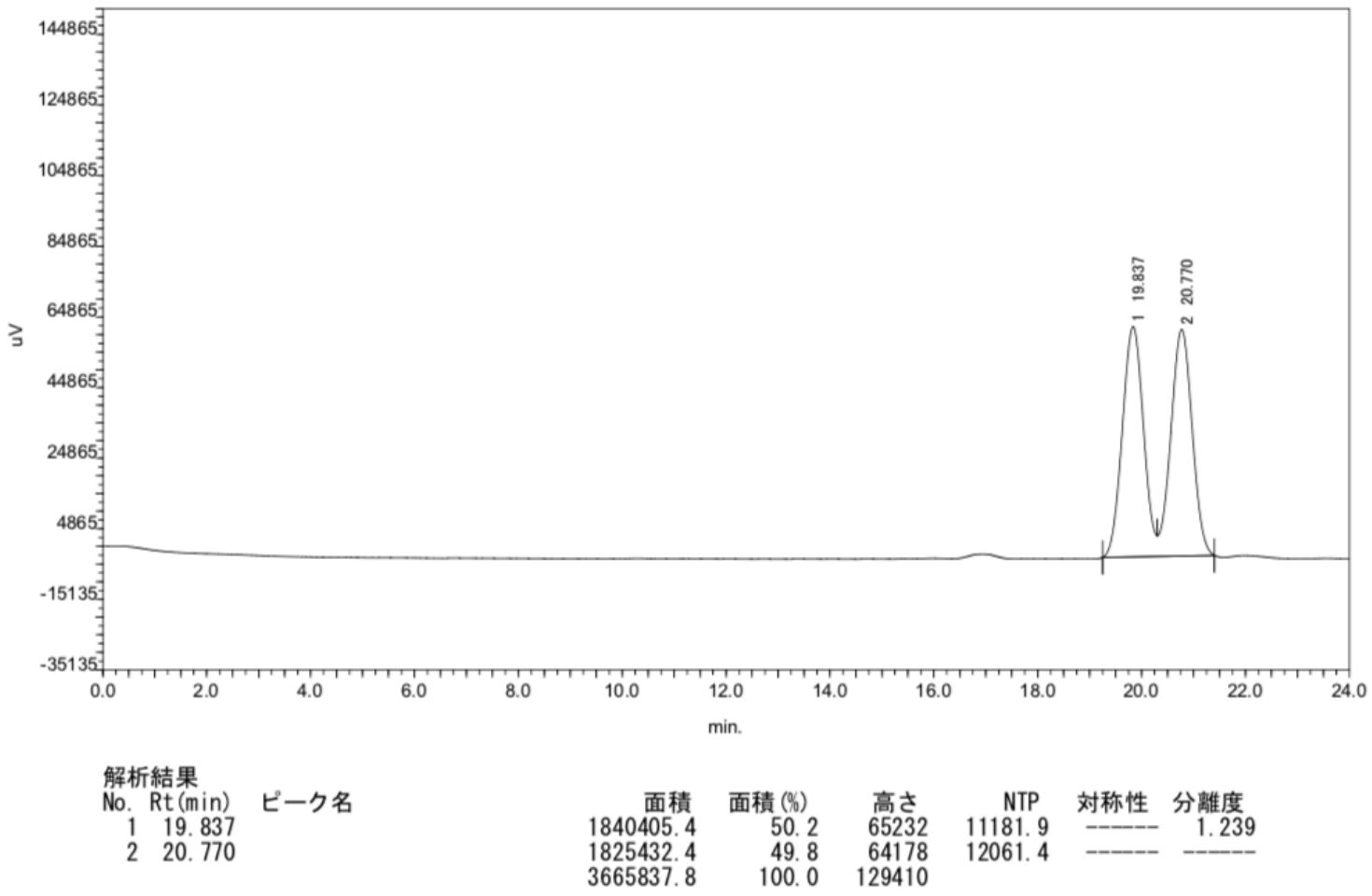




\section{Chromatogram Report}
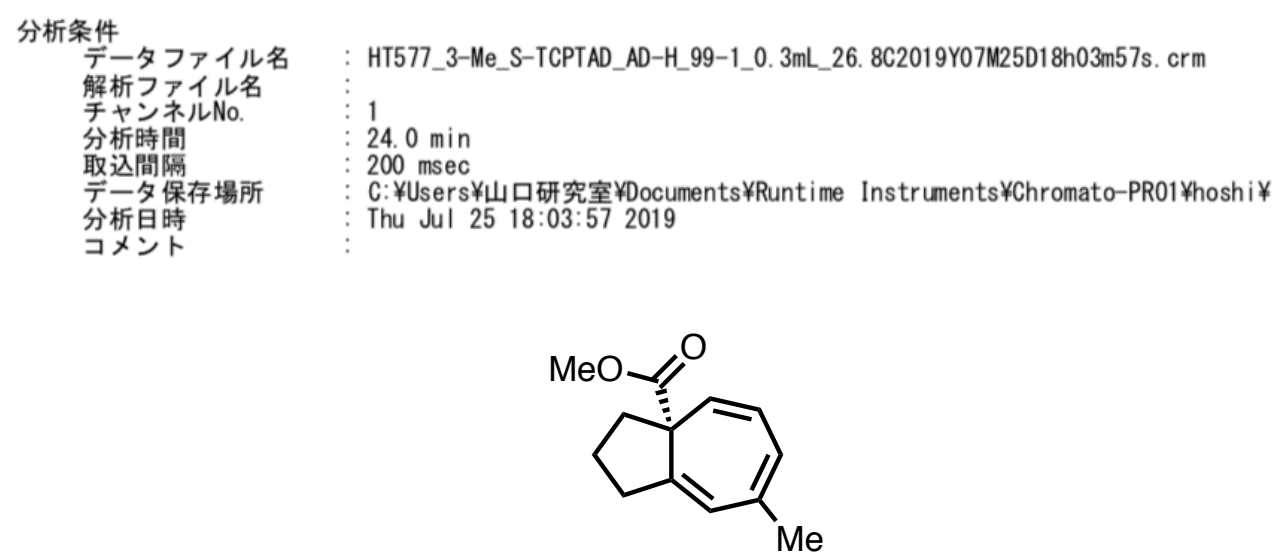

8B

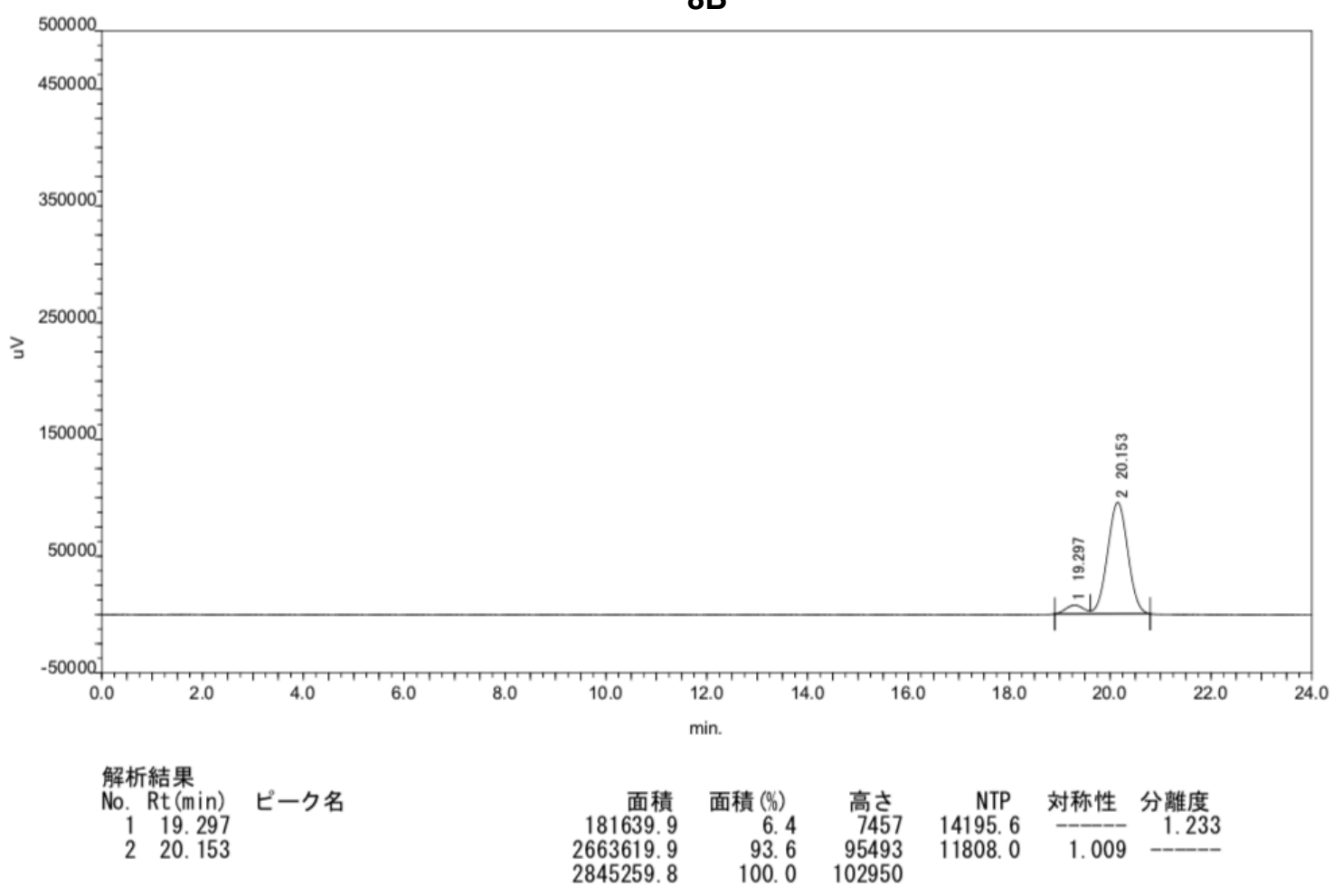




\section{Chromatogram Report}
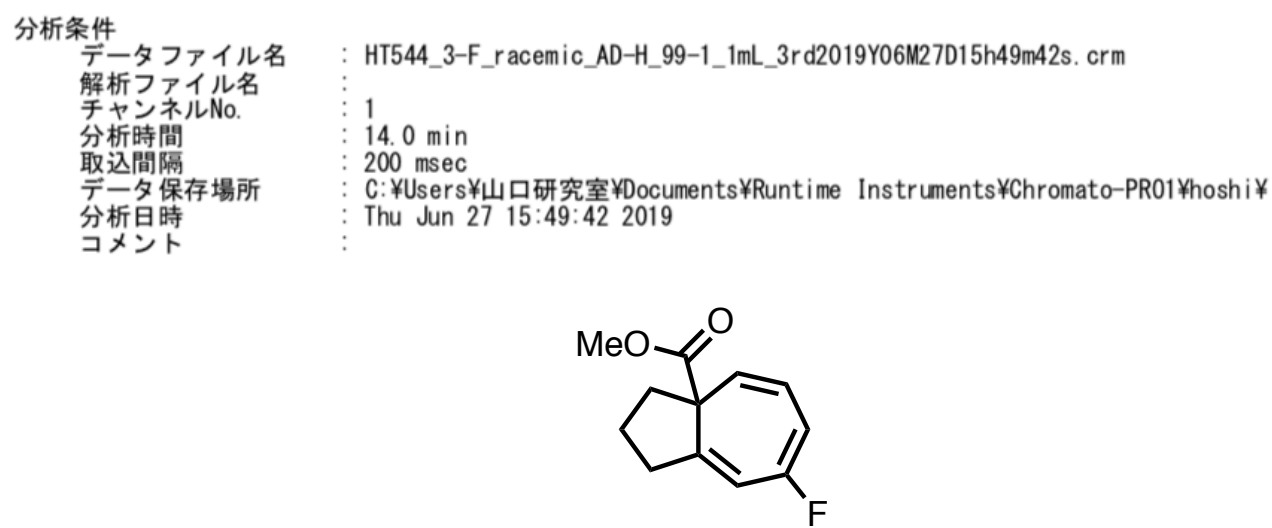

racemic $8 \mathrm{C}$

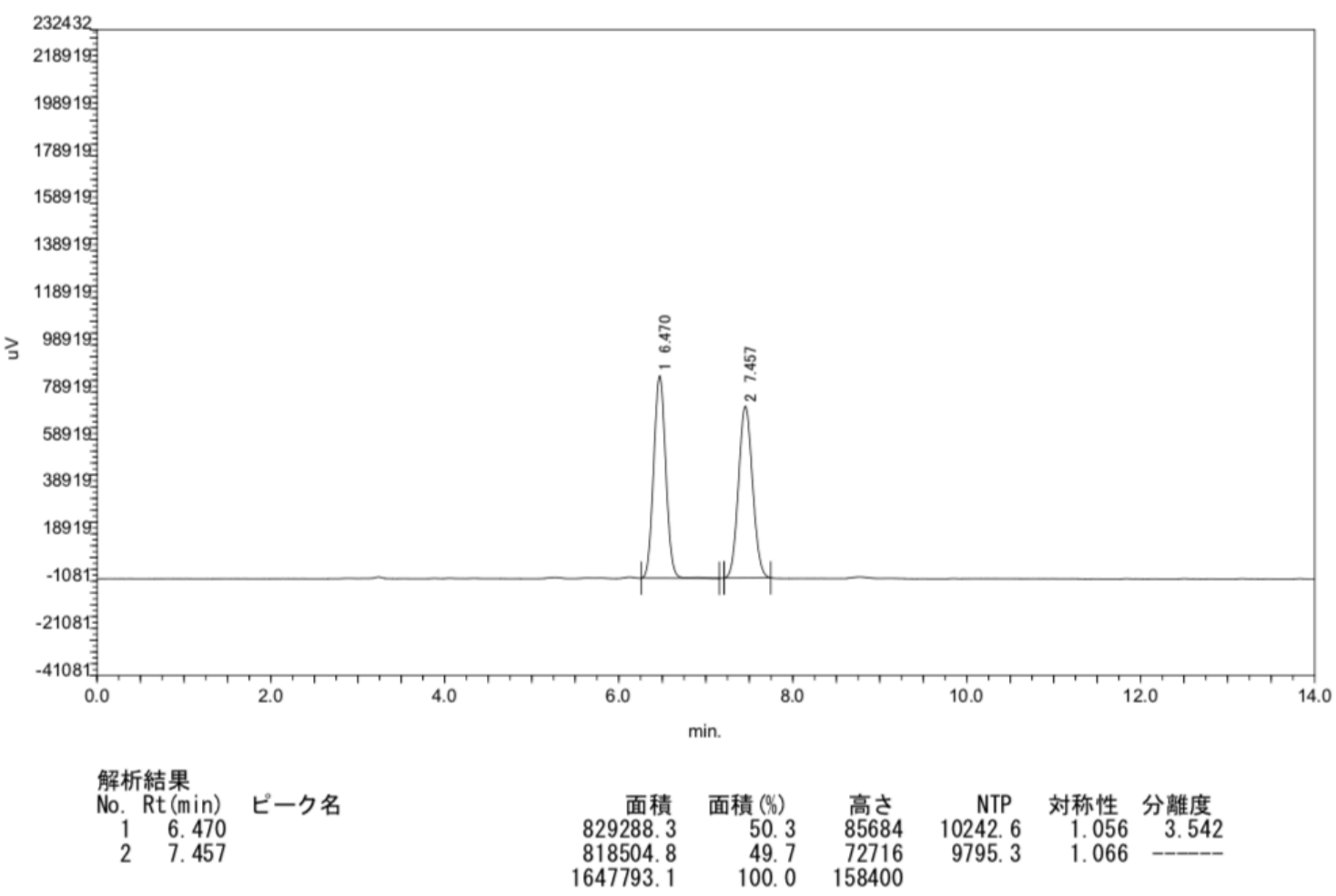




\section{Chromatogram Report}
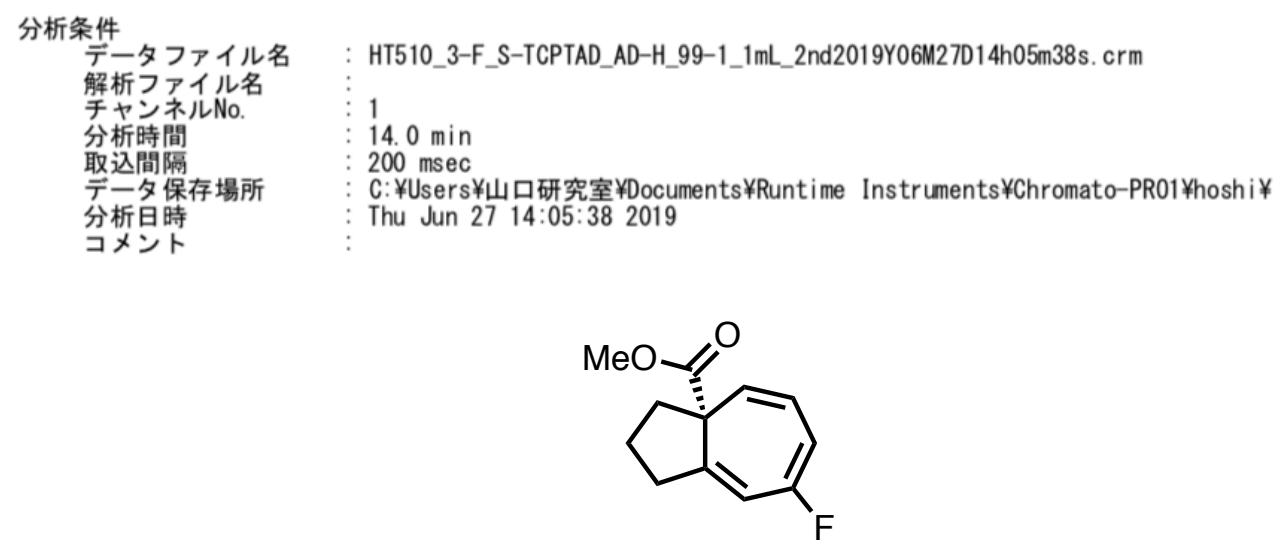

$8 \mathrm{C}$

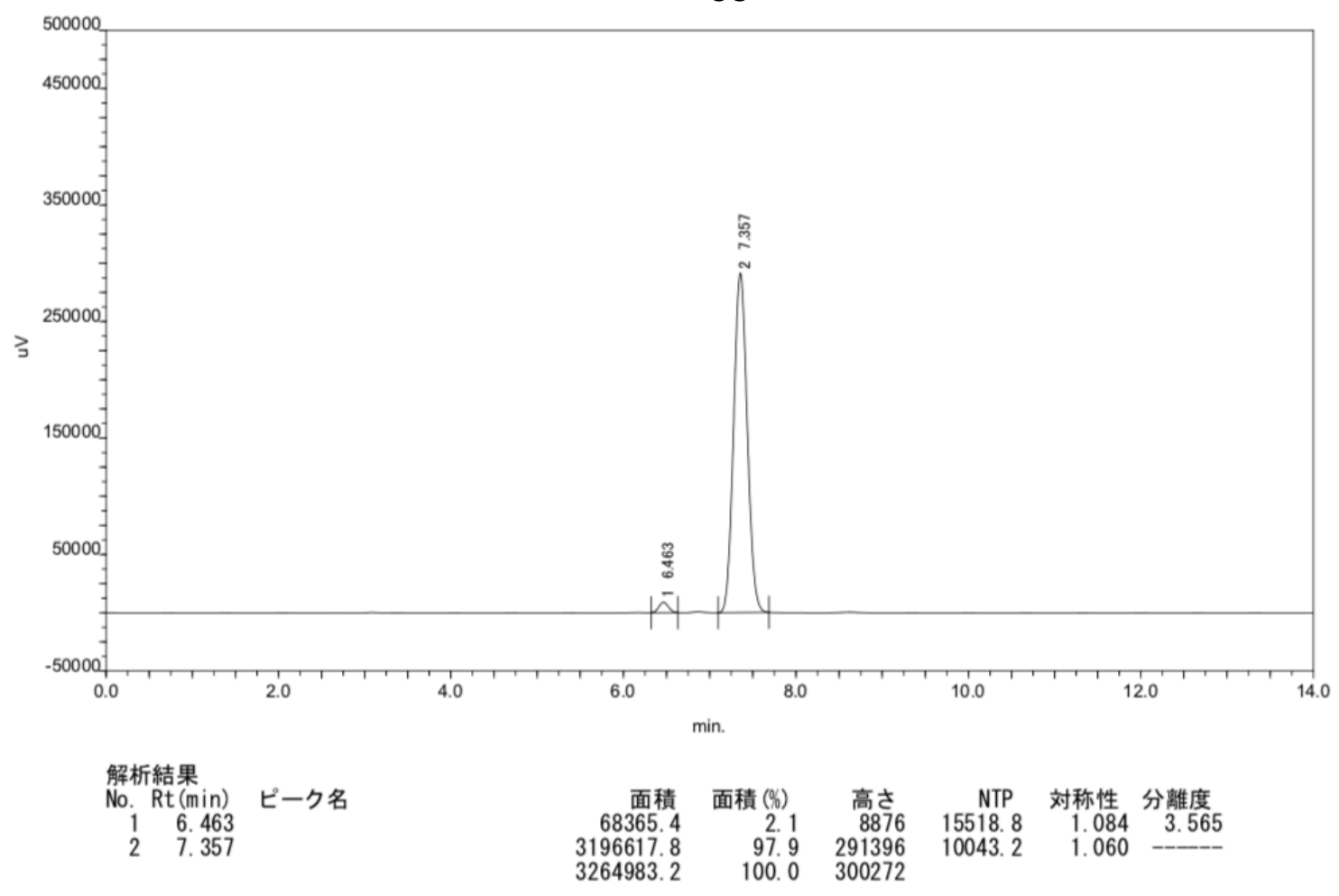




\section{Chromatogram Report}

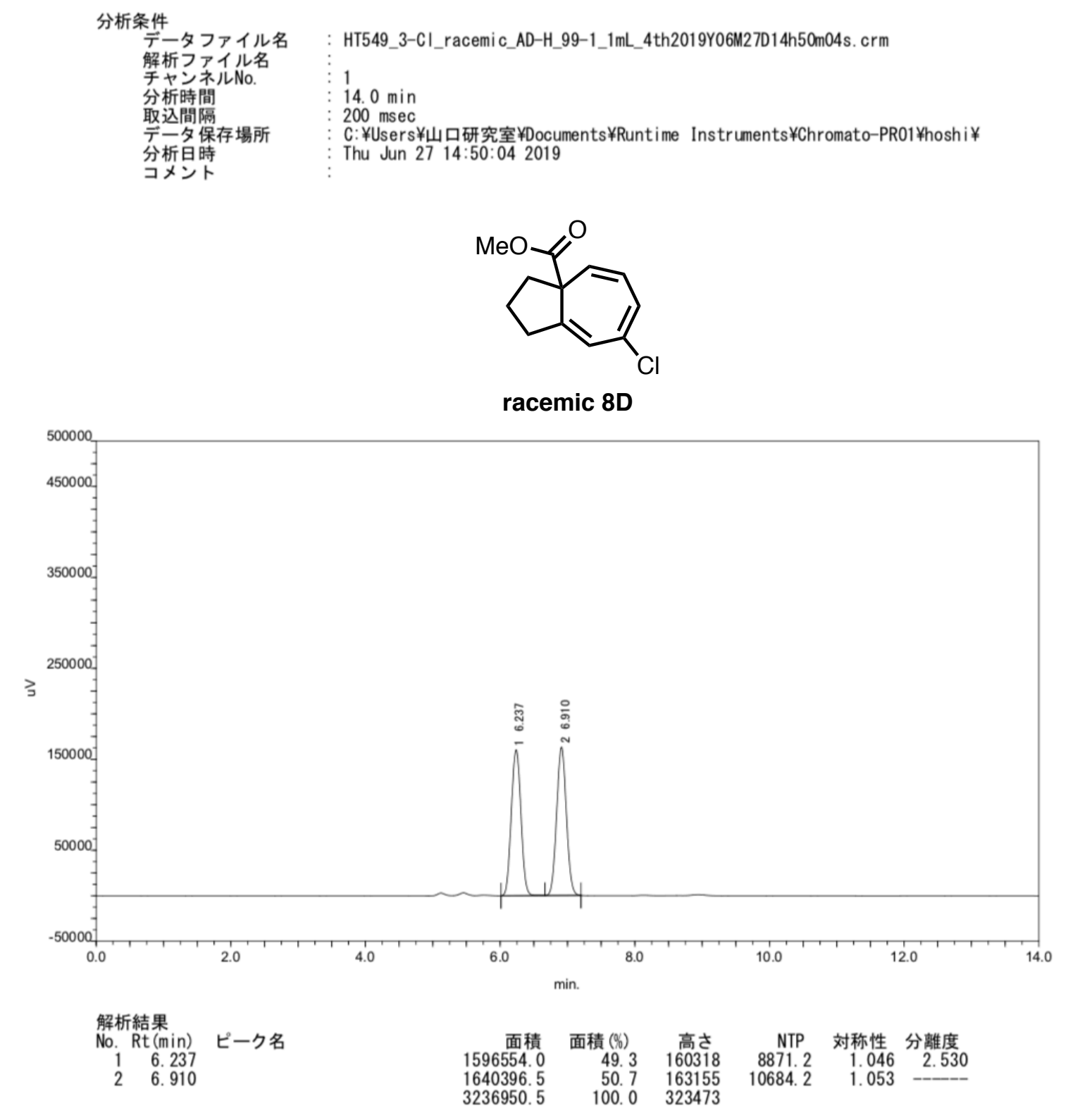




\section{Chromatogram Report}

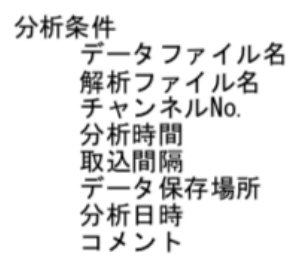

: HT545_3-CI_S-TCPTAD_AD-H_99-1_1mL2019Y06M26D17h56m49s.crm $14.0 \mathrm{~min}$ $200 \mathrm{msec}$

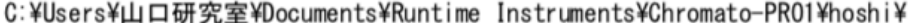
Wed Jun 26 17:56:49 2019

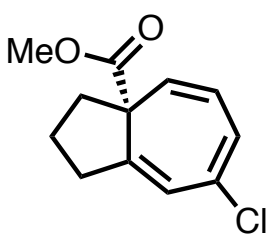

8D

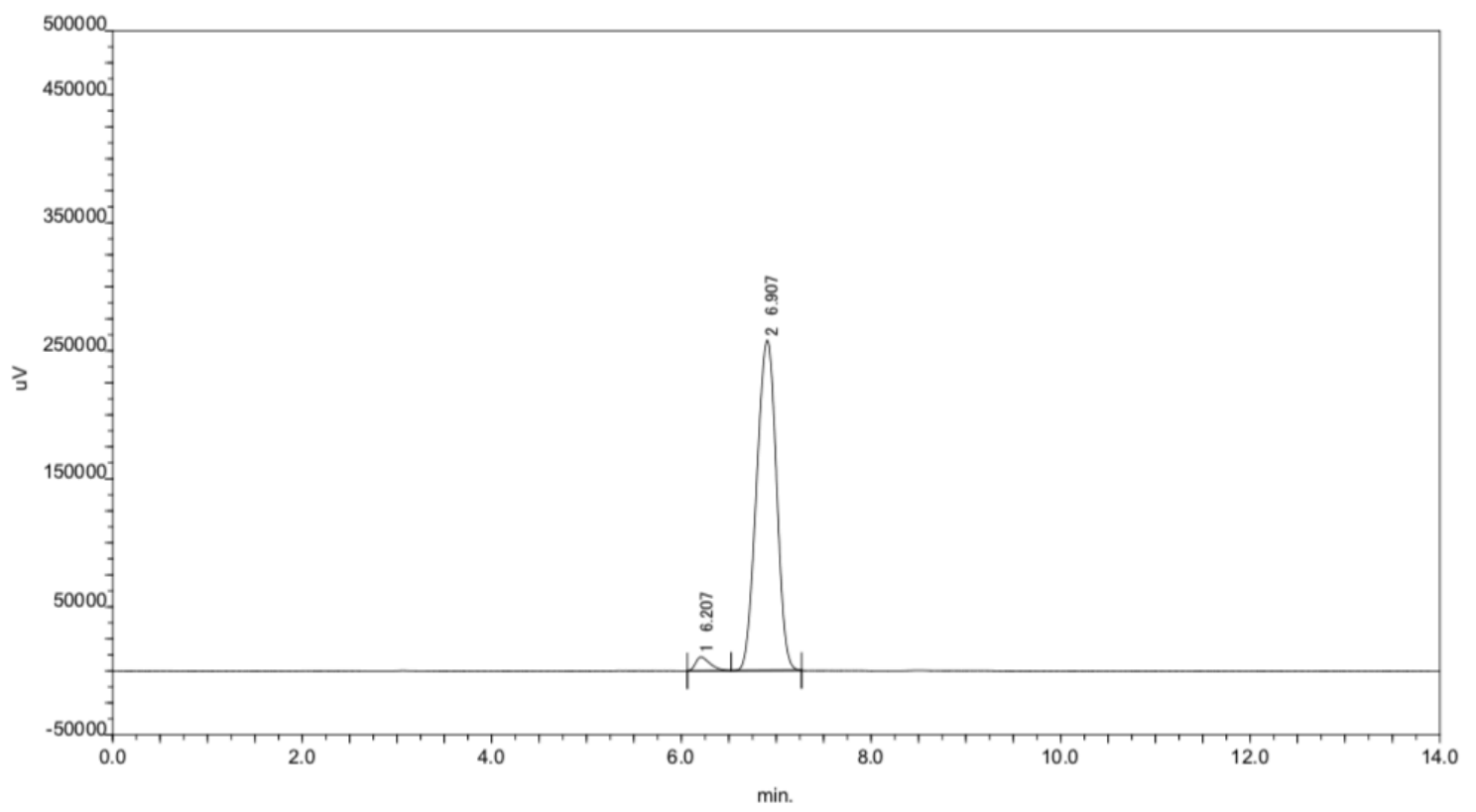

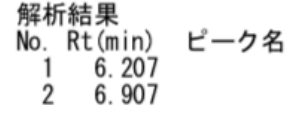

\begin{tabular}{rrr} 
面積 & \multicolumn{1}{c}{ 面積(\%) } & \multicolumn{1}{c}{ 高さ } \\
112664.9 & 10704 \\
3756321.8 & 97.1 & 257941 \\
3868986.2 & 100.0 & 268645
\end{tabular}

NTP 対称性 分離度

$\begin{array}{lll}7865.3 & 1.563 & 2.098\end{array}$

$5087.9 \quad 0.960$ 


\section{Chromatogram Report}
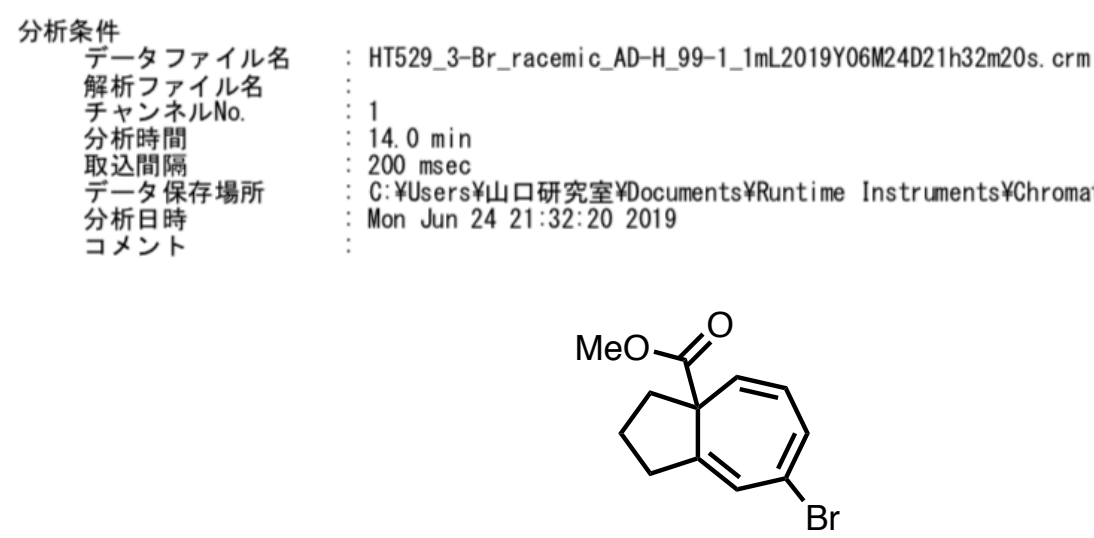

racemic $8 \mathrm{E}$

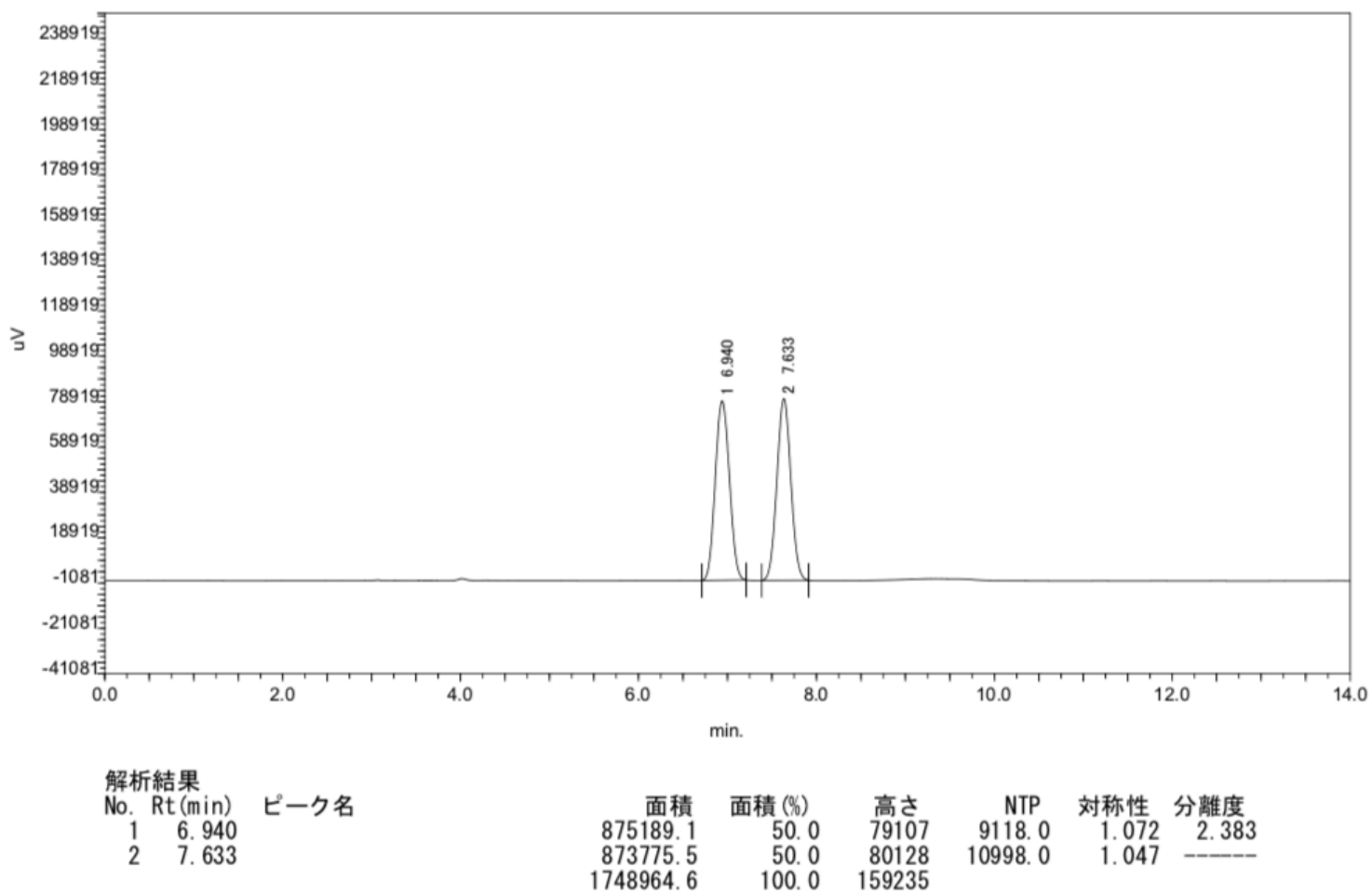




\section{Chromatogram Report}
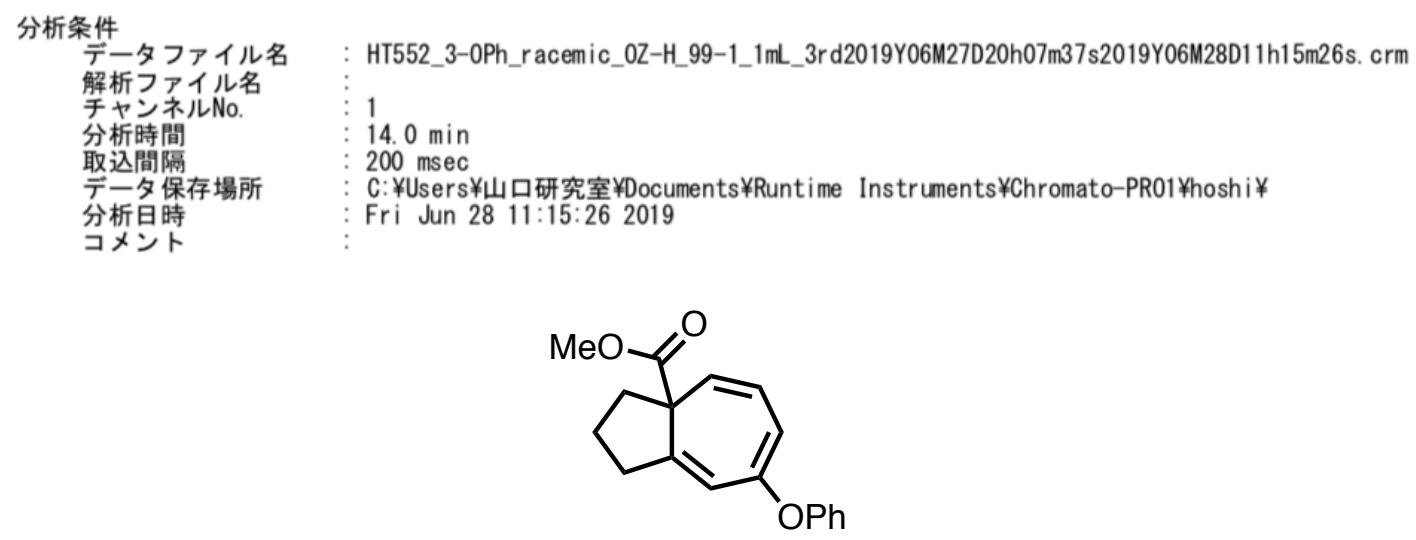

racemic $8 F$

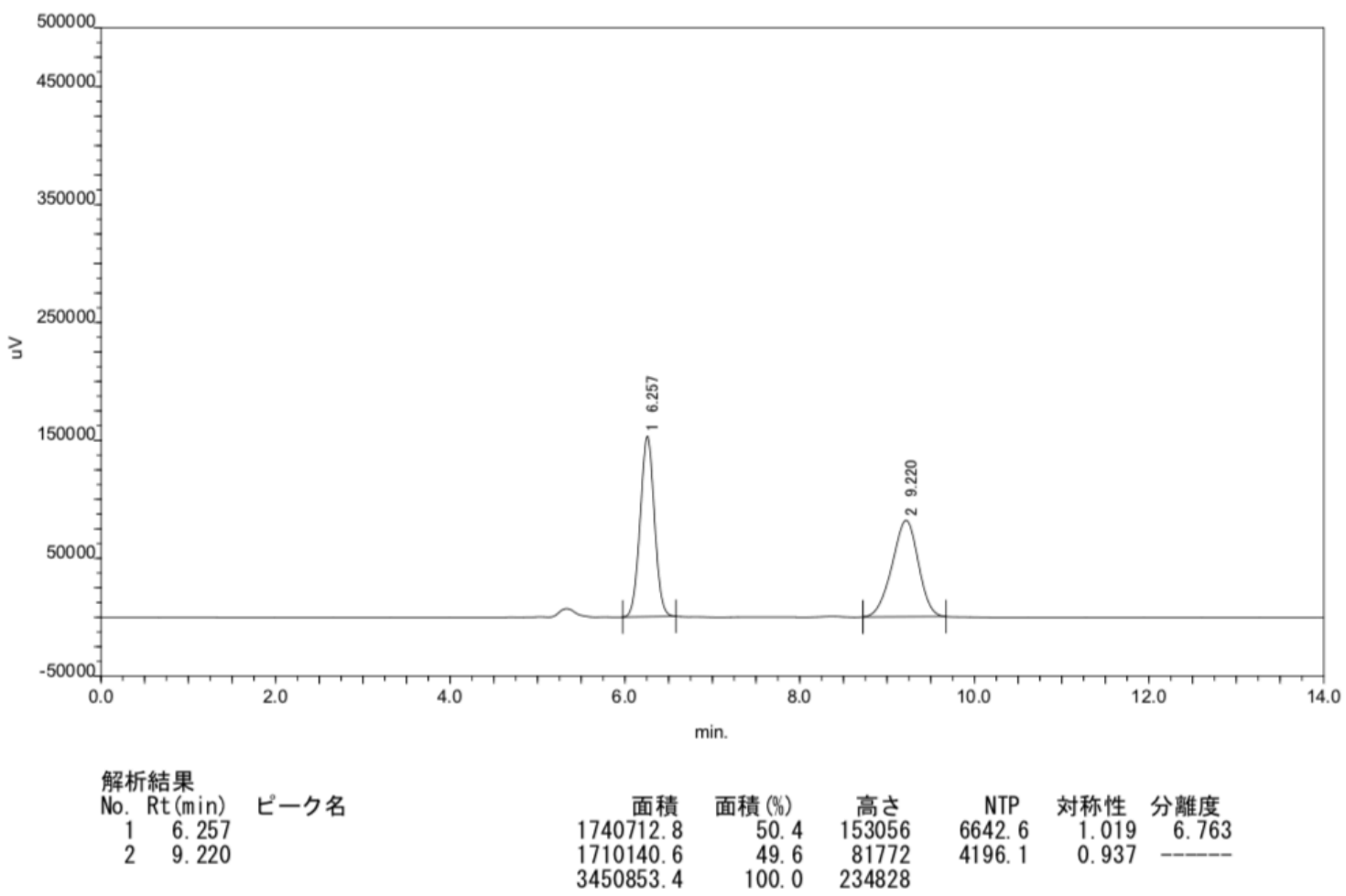




\section{Chromatogram Report}

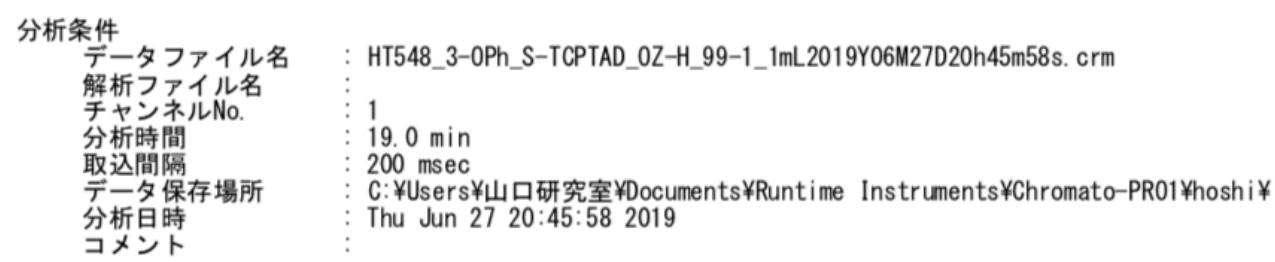

C: $¥$ Users ¥山口研究室 $¥ D o c u m e n t s ¥ R u n t i m e$ Instruments $¥$ Chromato-PR01 thoshi $¥$

Thu Jun 27 20:45:58 2019

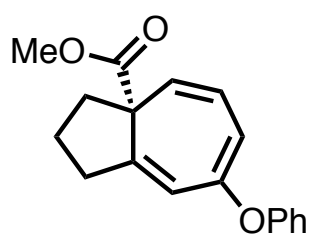

$8 \mathrm{~F}$

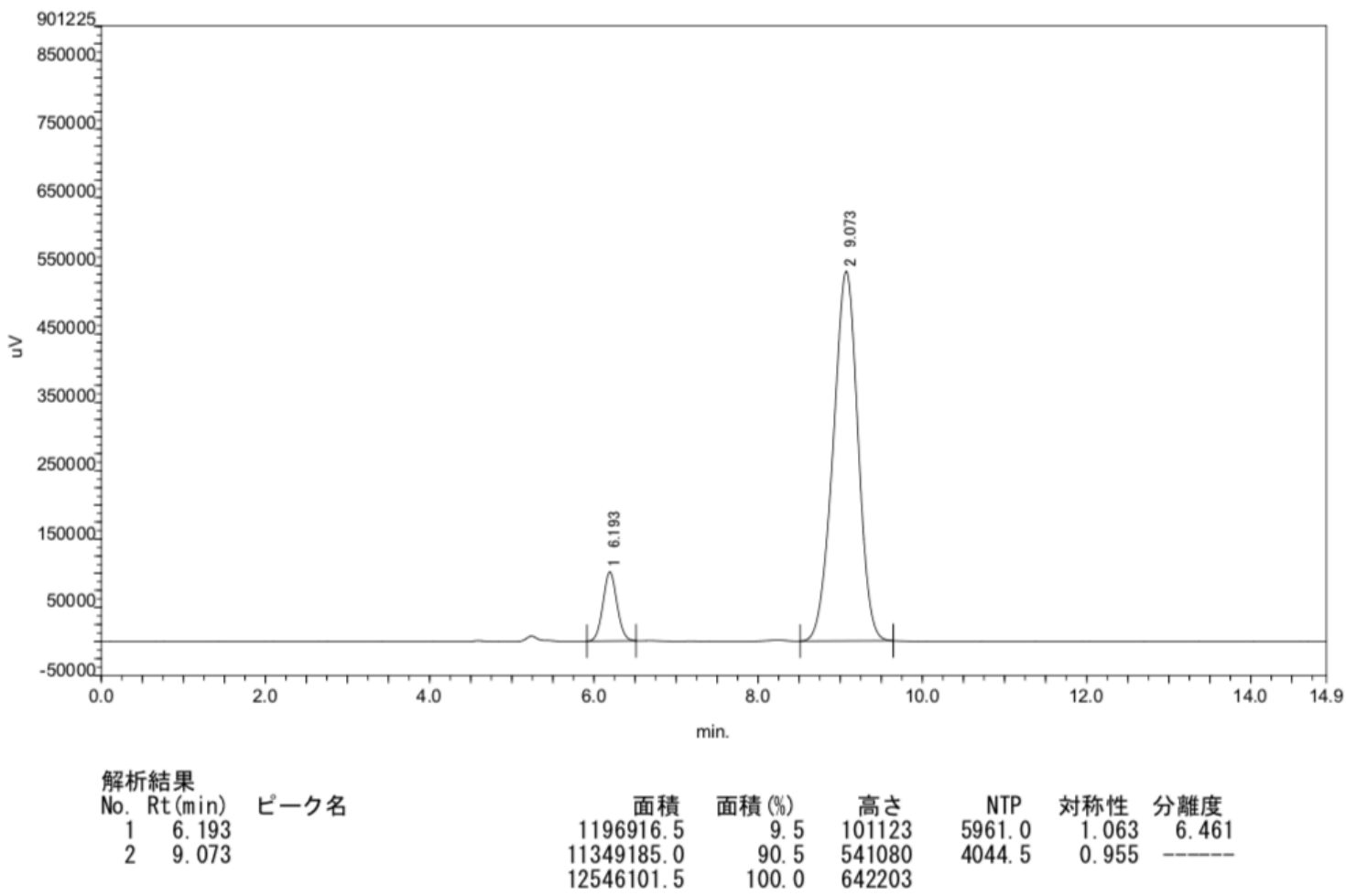




\section{Chromatogram Report}
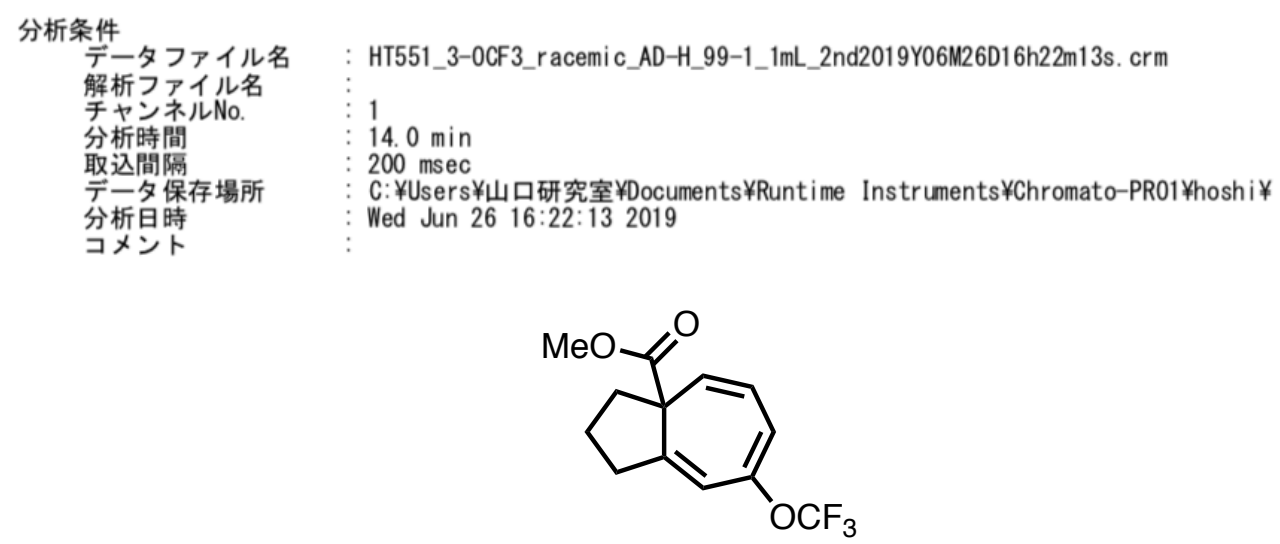

racemic $8 \mathrm{G}$

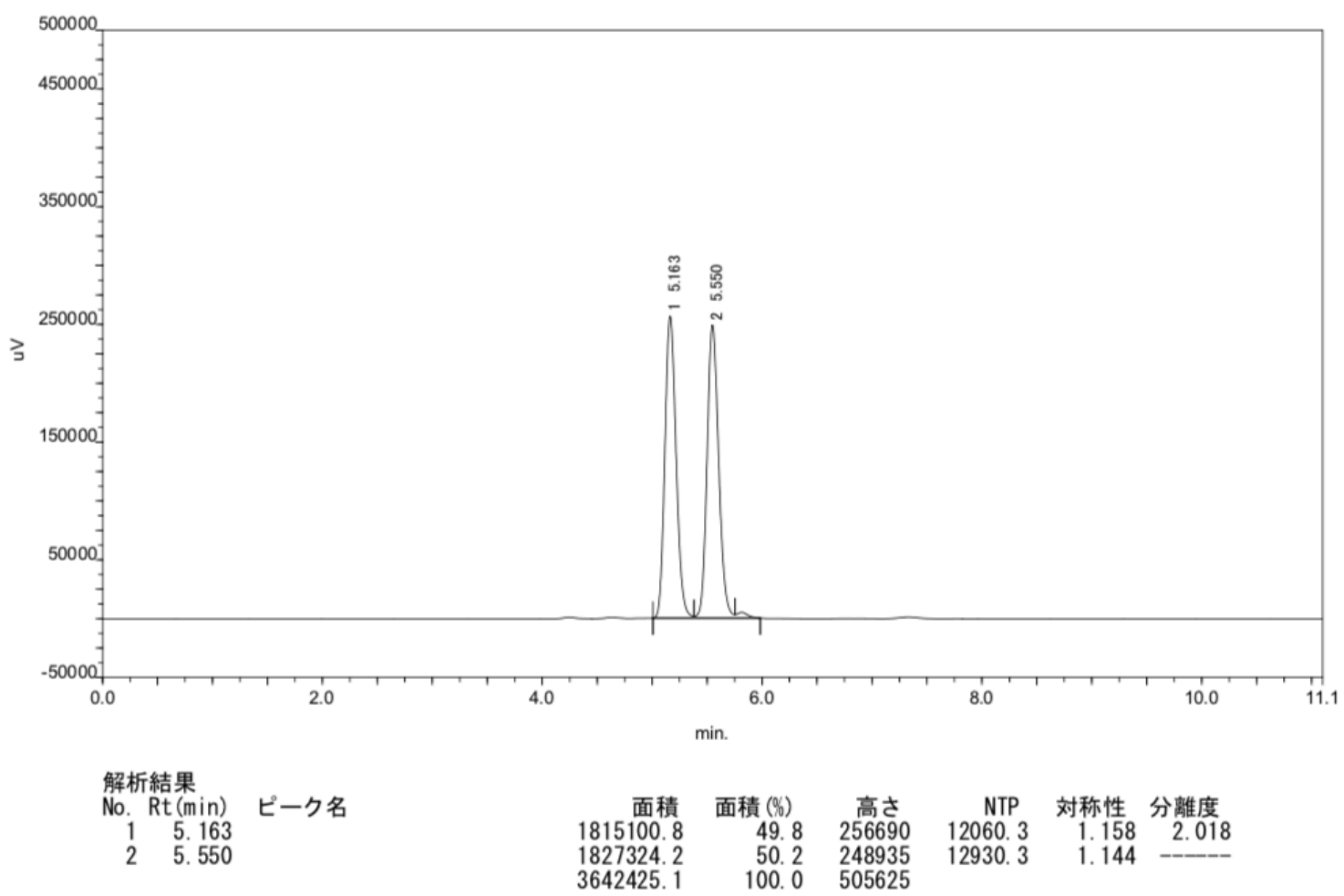




\section{Chromatogram Report}

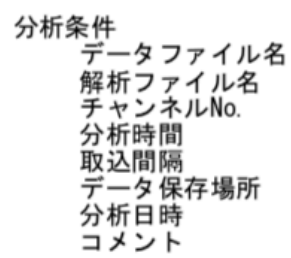

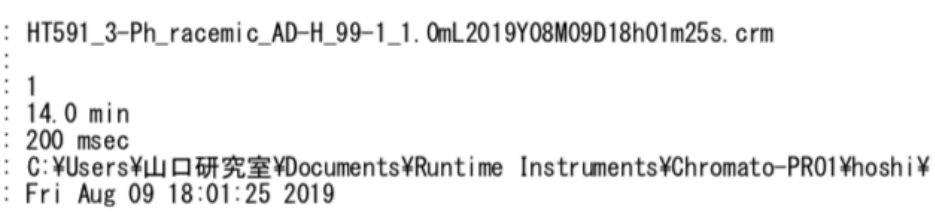

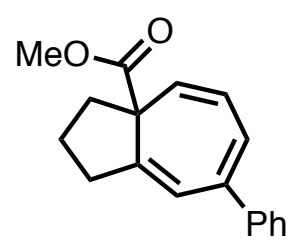

racemic $8 \mathrm{H}$

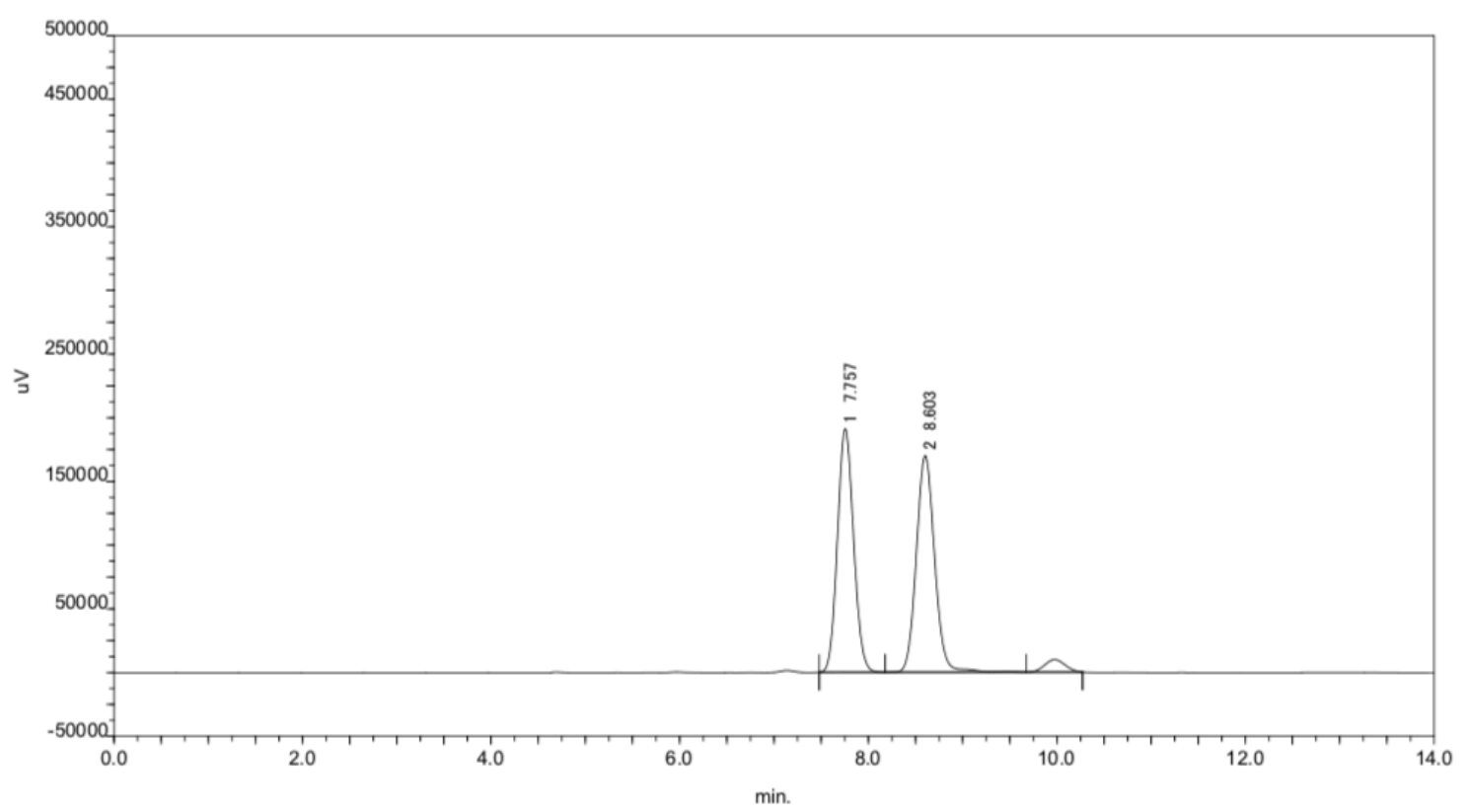

\begin{tabular}{rrrrrr} 
面皘 & \multicolumn{1}{c}{ 面積(\%) } & \multicolumn{1}{c}{ 高さ } & \multicolumn{1}{c}{ NTP } & 対称性 & 分離度 \\
2275231.2 & 49.7 & 191079 & 9598.6 & 1.085 & 2.504 \\
2299150.2 & 50.3 & 170026 & 9156.1 & 1.087 & --- \\
4574381.3 & 100.0 & 361105 & & &
\end{tabular}




\section{Chromatogram Report}

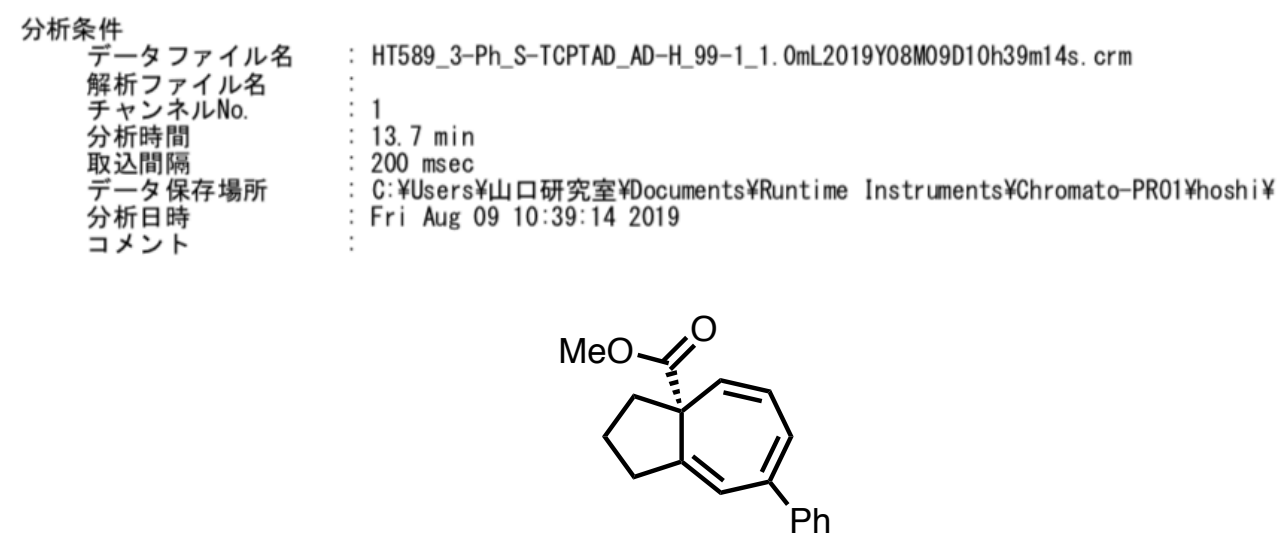

$8 \mathrm{H}$

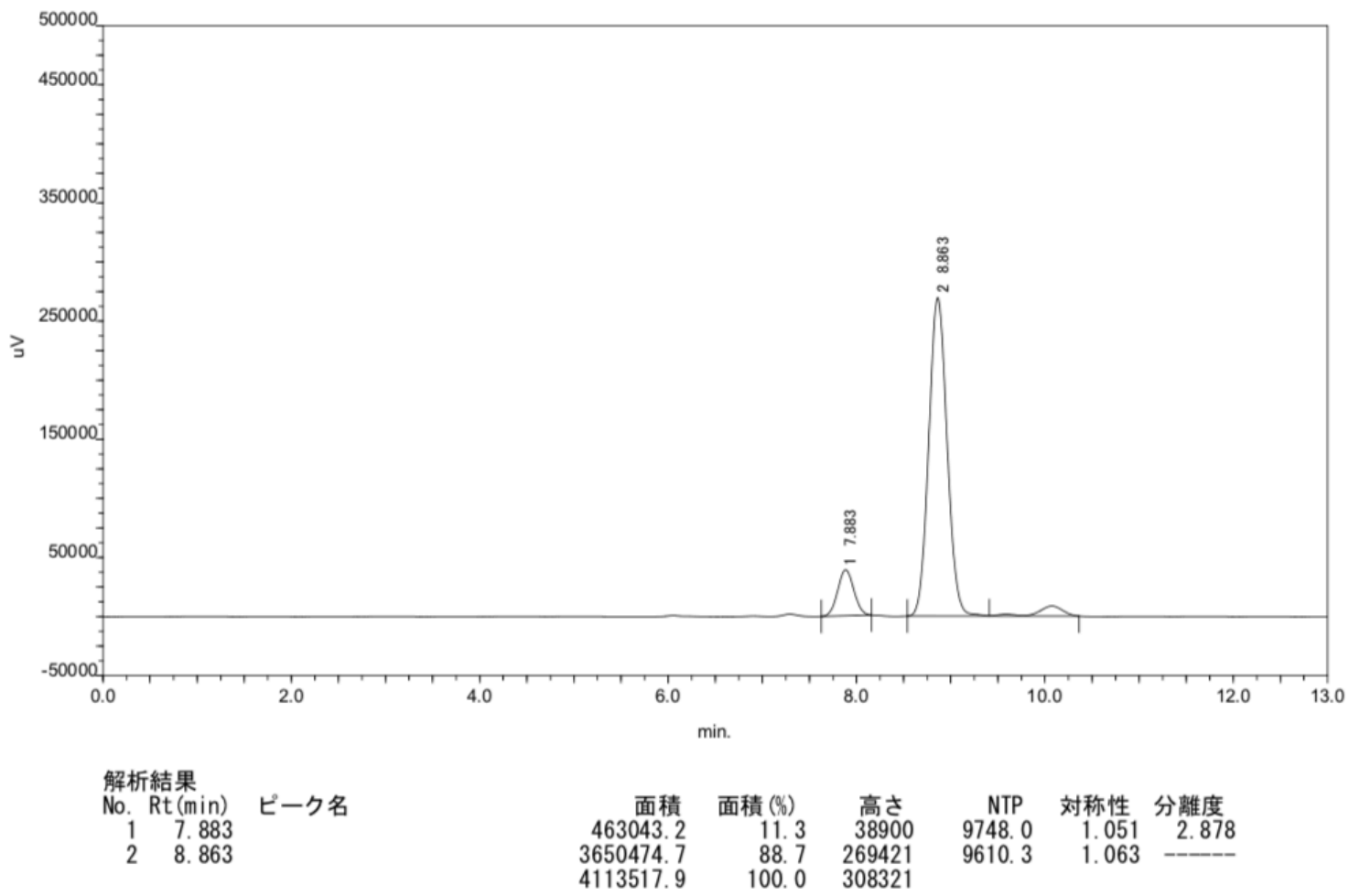




\section{Chromatogram Report}

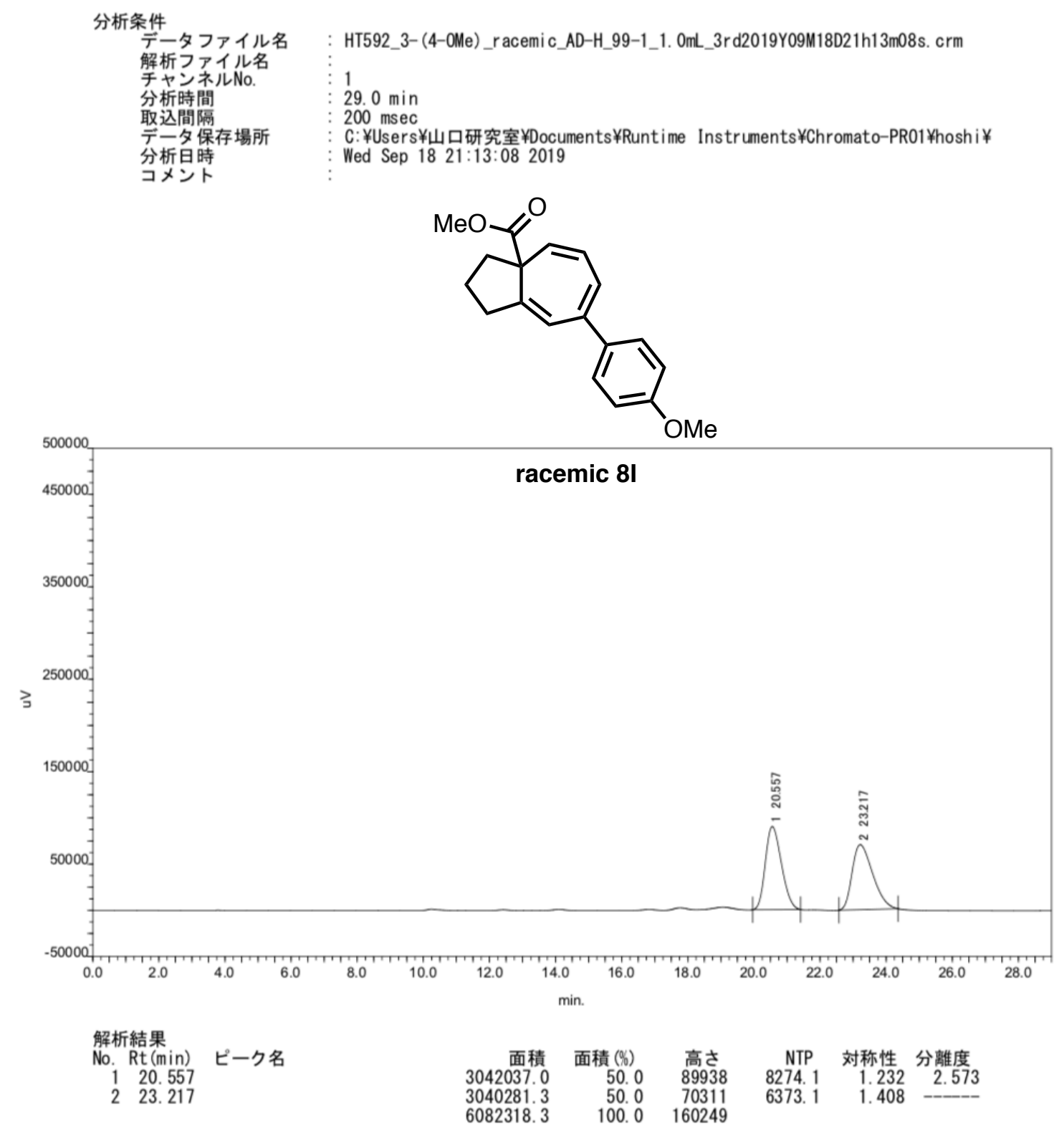




\section{Chromatogram Report}

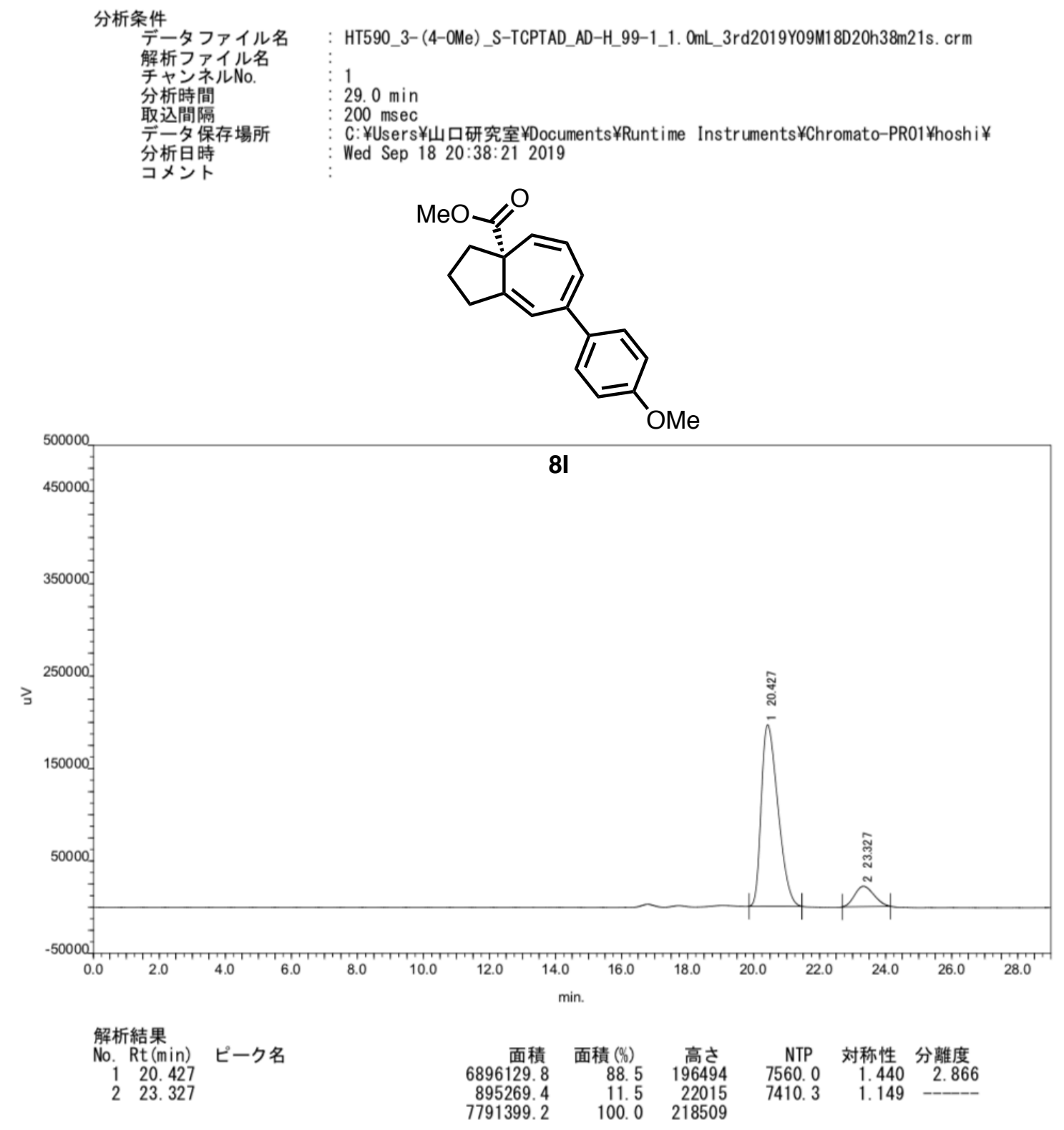


Supporting Information (Hoshi, Ota, Inokuma, Yamaguchi) Asymmetric Synthesis of 5,7-Fused Ring System Enabled by Intra-molecular Buchner with Chiral Rhodium Catalyst

\section{Chromatogram Report}

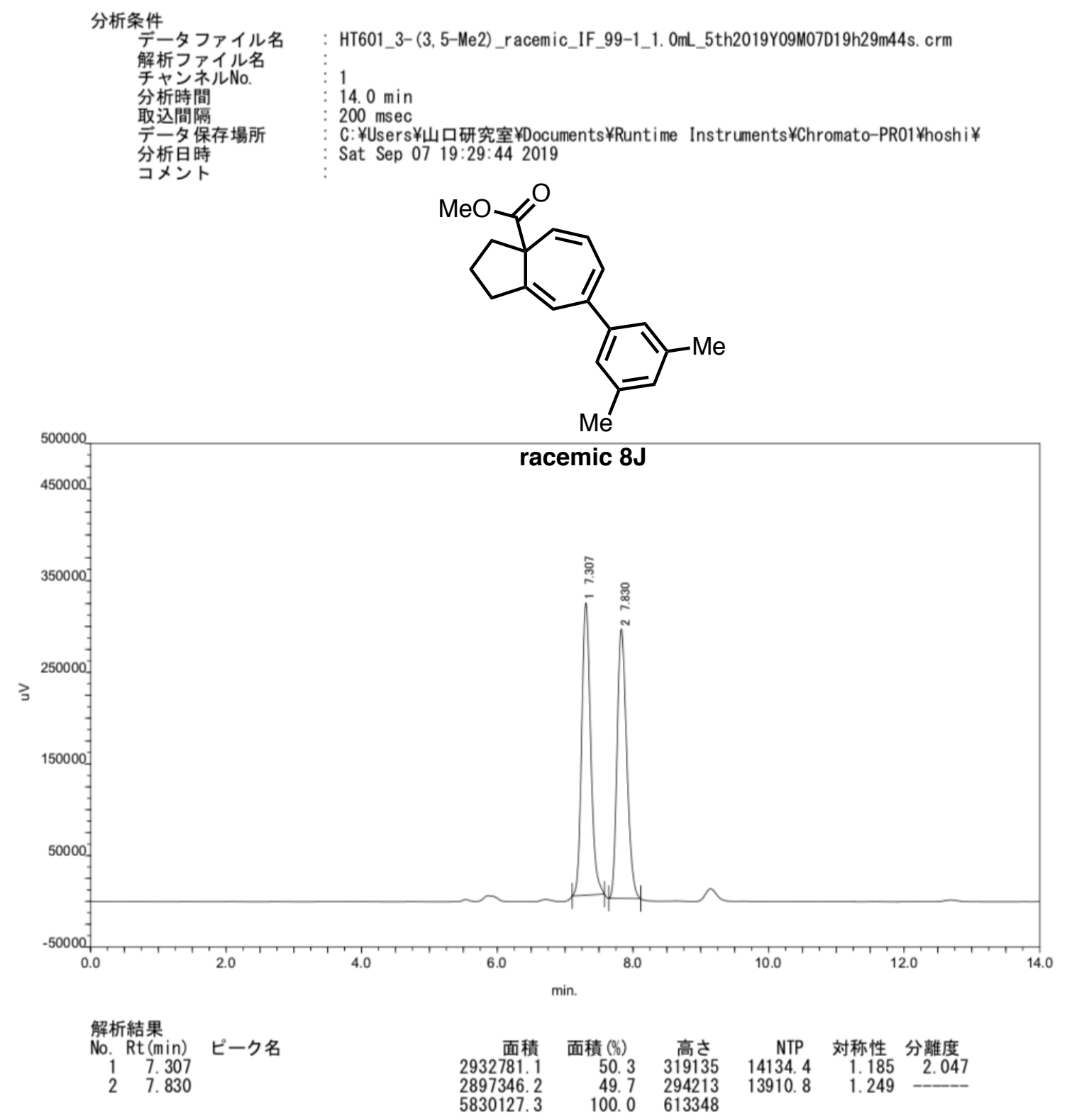


Supporting Information (Hoshi, Ota, Inokuma, Yamaguchi) Asymmetric Synthesis of 5,7-Fused Ring System Enabled by Intra-molecular Buchner with Chiral Rhodium Catalyst

\section{Chromatogram Report}

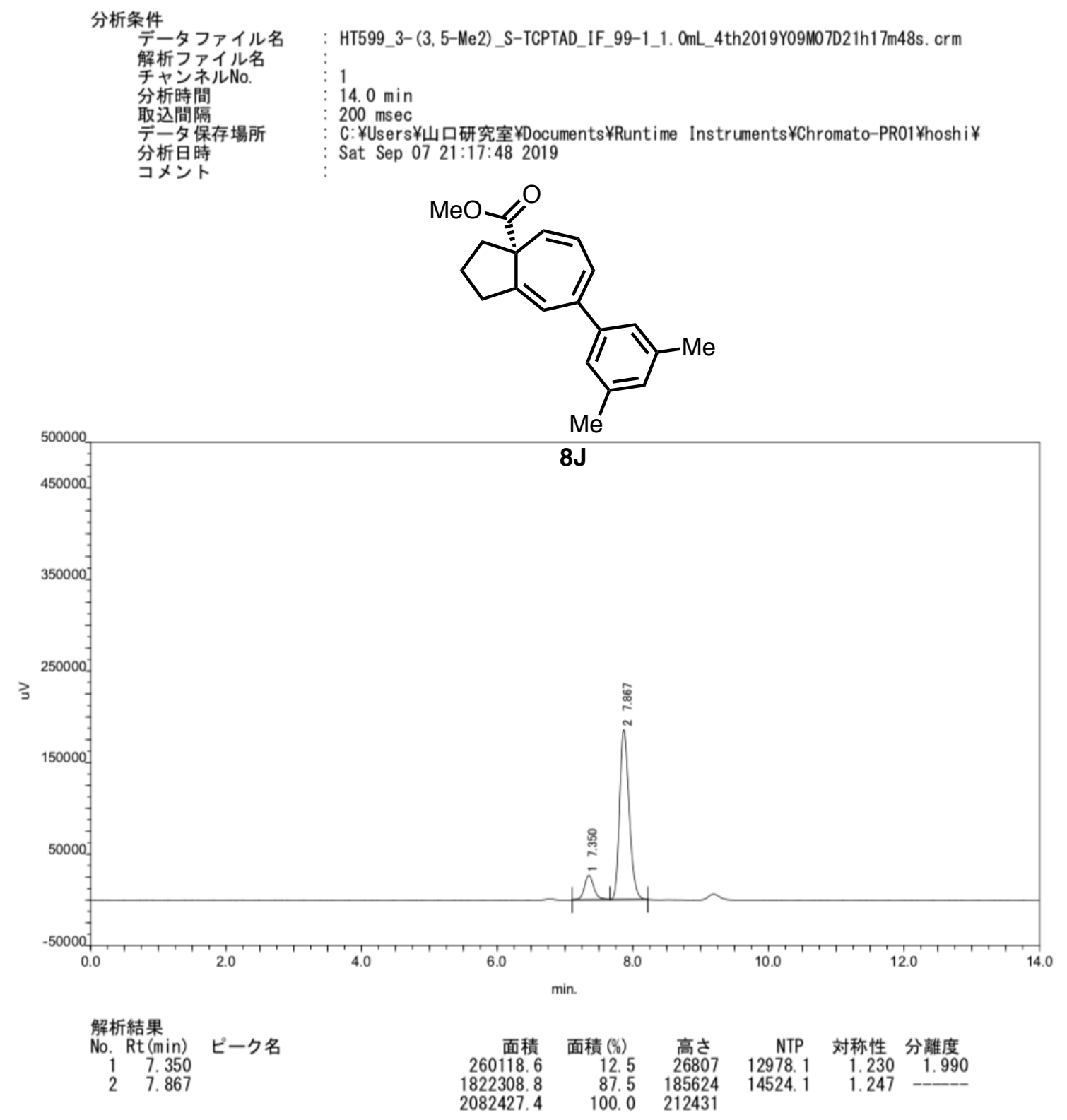




\section{Chromatogram Report}

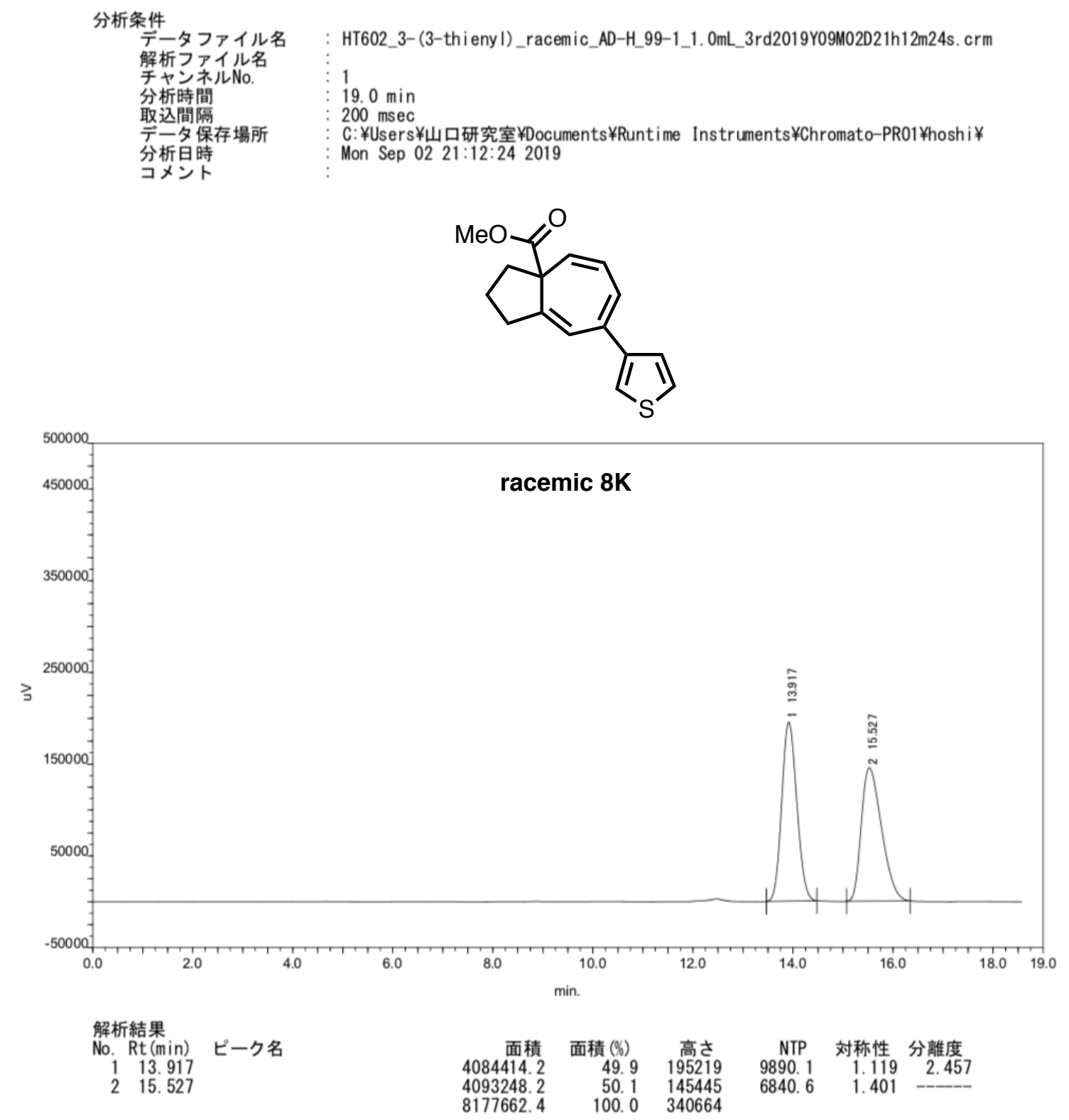




\section{Chromatogram Report}

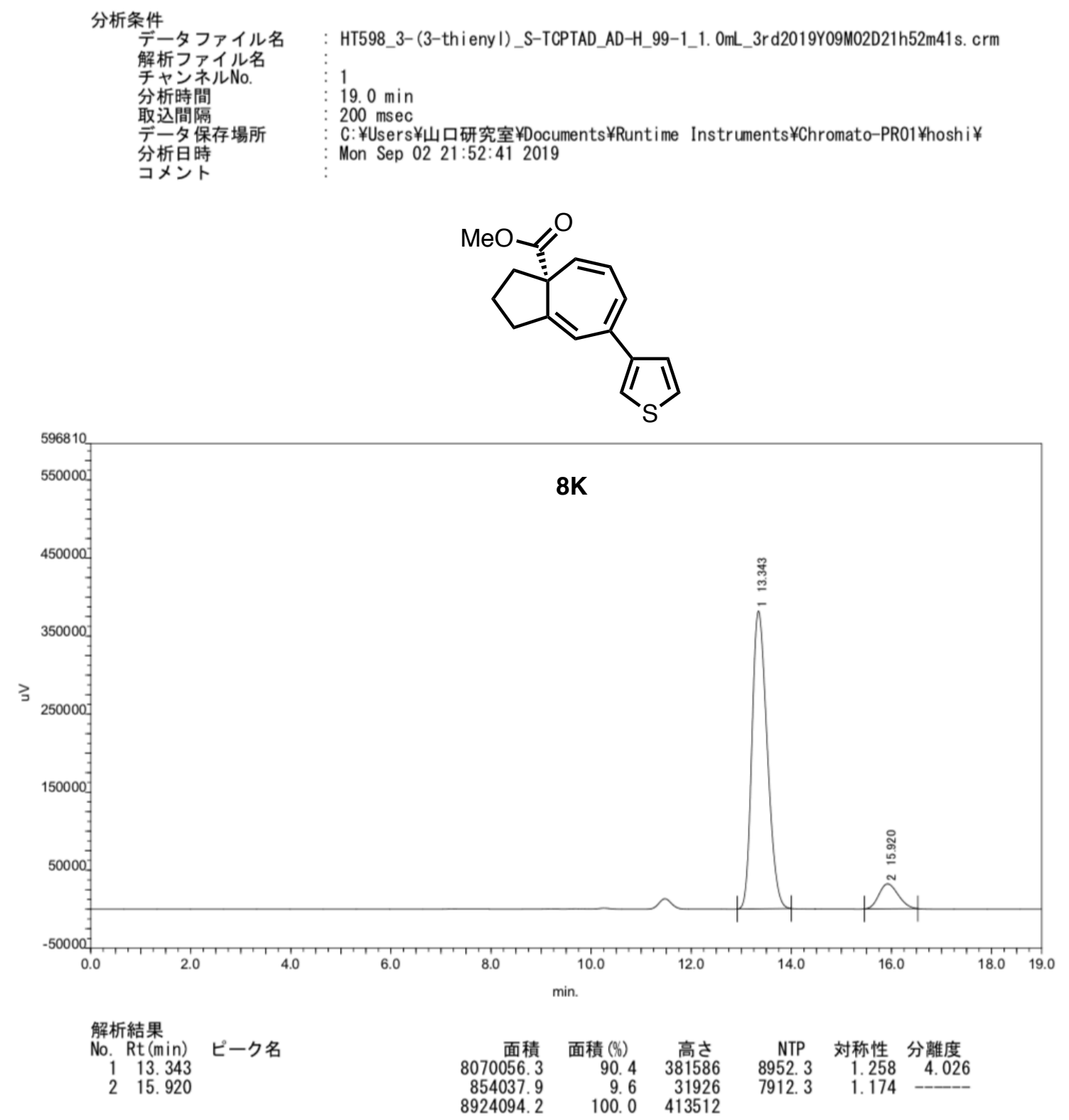




\section{Single Crystal X-ray Analysis}

Single crystal X-ray diffraction data were collected on Rigaku XtaLAB Synergy-R/DW (for compound 2A) equipped with a HyPix-6000HE detector or Rigaku XtaLAB P200 (for compound 8E) diffractometers equipped with a PILATUS200K detector using multi-layer mirror $\left(\mathrm{CuK}_{\alpha}\right.$ radiation $\lambda=$ $1.5418 \AA$ ). All structures were solved using a dual-space algorithm (SHELXT ${ }^{[7]}$ ) and refined using full-matrix least-squares method (SHELXL ${ }^{[8]}$ ). All the non-hydrogen atoms for host framework were refined anisotropically.

\section{Crystallization of Compound $2 \mathrm{~A}$}

Compound $2 \mathrm{~A}$ was crystallized by cooling as-synthesized sample at $0{ }^{\circ} \mathrm{C}$ in a refrigerator. Because crystals of $\mathbf{2 A}$ melt at room temperature, a single crystal for the X-ray measurement was immediately picked and mounted onto a diffractometer.

\section{Crystallographic data for $\mathbf{2 A}$}

$\left[\mathrm{C}_{12} \mathrm{H}_{14} \mathrm{O}_{2}\right], M=190.23$, crystal size: $0.28 \times 0.16 \times 0.09 \mathrm{~mm}^{3}$, Monoclinic, space group $P 2_{1}, a=$ $8.06850(10) \AA, b=6.08350(10) \AA, c=10.32580(10) \AA, \beta=92.9420(10)^{\circ}, V=506.171(12) \AA^{3}, Z=2$, $T=123(2) \mathrm{K}, \mu=0.670 \mathrm{~mm}^{-1}, D_{\text {calc }}=1.248 \mathrm{~g} / \mathrm{cm}^{3}, 4.287^{\circ} \leq \theta \leq 72.433^{\circ}, 1925$ unique reflections out of 1926 with $I>2 \sigma(I), \mathrm{GOF}=1.077, R_{1}=0.0293$ and $w R_{2}=0.0768$, Flack parameter (Parsons) $=$ 0.08(9), CCDC deposit number 1962381.

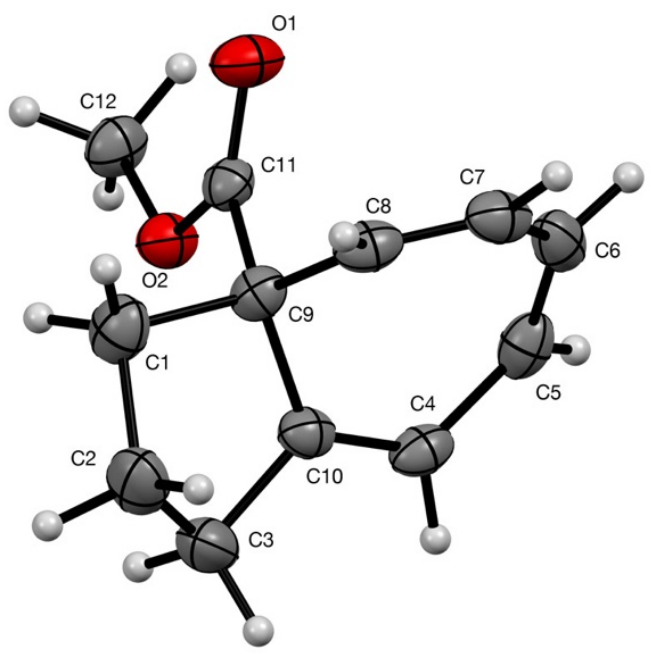

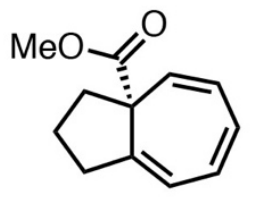

2A

Figure S1 ORTEP drawing of compound $\mathbf{2 A}$ at the $50 \%$ probability level. 


\section{Crystallization of Compound $8 \mathrm{E}$}

Compound $\mathbf{8 E}$ was melted at $110^{\circ} \mathrm{C}$ in a glass vial using oil bath. Single crystals were grown upon slow cooling to room temperature.

\section{Crystallographic data for $8 \mathrm{E}$}

[ $\left.\mathrm{C}_{12} \mathrm{H}_{13} \mathrm{BrO}_{2}\right], M=269.13$, crystal size: $0.20 \times 0.12 \times 0.06 \mathrm{~mm}^{3}$, Hexagonal, space group $P 6_{5}, a=b$ $=7.74700(10) \AA, c=32.3192(4) \AA, \alpha=\beta=90^{\circ}, \gamma=120^{\circ}, V=1679.80(5) \AA^{3}, Z=6, T=123(2) \mathrm{K}, \mu=$ $4.820 \mathrm{~mm}^{-1}, D_{\text {calc }}=1.596 \mathrm{~g} / \mathrm{cm}^{3}, 6.598^{\circ} \leq \theta \leq 70.432^{\circ}, 2098$ unique reflections out of 2125 with $I>$ $2 \sigma(I), \mathrm{GOF}=1.085, R_{1}=0.0230$ and $w R_{2}=0.0596$, Flack parameter (Parsons) $=-0.015(9), \mathrm{CCDC}$ deposit number 1962382.

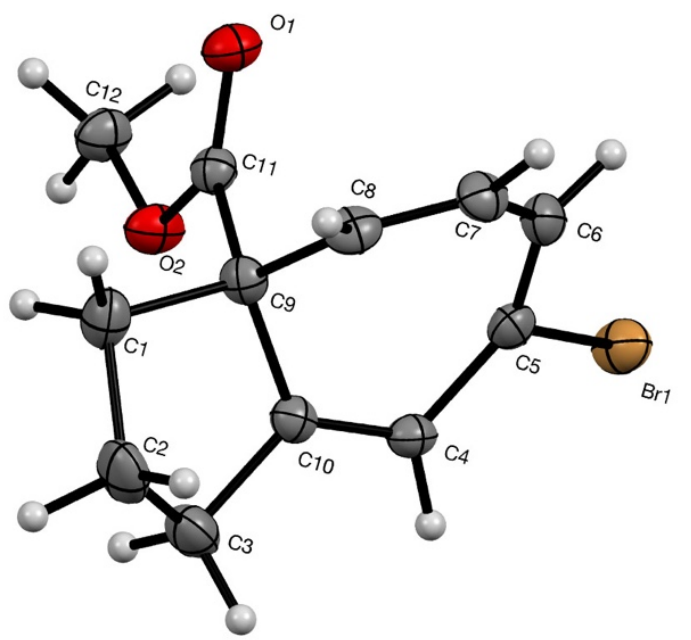

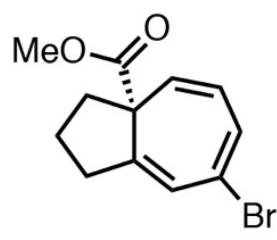

$8 \mathrm{E}$

Figure S2 ORTEP drawing of compound $\mathbf{8 E}$ at the $50 \%$ probability level. 


\section{References}

[1] Alvariño, C.; Simond, D.; Lorente, P. M.; Besnard, C.; Williams, A. F. Chains, Necklaces and Weaving Chain-link Grids from Self-Assembly Reactions. Chem. Eur. J. 2015, 21, 8851-8858.

[2] Reddy, R. P.; Davies, H. M. L. Dirhodium Tetracarboxylates Derived from Adamantylglycine as Chiral Catalysts for Enantioselective C-H Aminations. Org. Lett. 2006, 8, 5013-5016.

[3] Taber, D. F.; You, K.; Song, Y. A Simple Preparation of a-Diazo Esters. J. Org. Chem. 1995, 60, 1093.

[4] Miura, T.; Funakoshi, Y.; Murakami, M. Intramolecular Dearomatizing [3 + 2] Annulation of $\alpha$ Imino Carbenoids with Aryl Rings Furnishing 3,4-Fused Indole Skeletons. J. Am. Chem. Soc. 2014, $136,2272-2275$.

[5] Baeckström, P.; Jacobsson, U.; Norin, T.; Unelius, C. R. Synthesis and Characterization of All Four Isomers of Methyl 2,4-Decadienoate for an Investigation of the Pheromone Components of Pityogenes chaicographus. Tetrahedron 1988, 44, 2541-2548.

[6] Lekkala, R.; Lekkala, R.; Moku, B.; Qin, H. L. $\mathrm{SO}_{2} \mathrm{~F}_{2}$ Mediated Dehydrative Cross-Coupling of Alcohols with Electron-Deficient Olefins in DMSO Using a Pd-Catalyst: One-Pot Transformation of Alcohols into 1,3-Dienes. Org. Chem. Front. 2019, 6, 796-800.

[7] Sheldrick, G. M. SHELXT - Integrated Space-Group and Crystal-Structure Determination. Acta Crystallogr. Sect. A 2015, 71, 3-8.

[8] Sheldrick, G. M. Crystal Structure Refinement with SHELXL. Acta Crystallogr. Sect. C 2015, 71, $3-8$. 